José Augusto Calvo Lonardoni

Desenvolvimento de um Simulador da

Mecânica Cardiovascular Humana controlada pelo Mecanismo Reflexo Baroceptor

Dissertação apresentada à Escola

Politécnica da Universidade de São Paulo para obtenção do título de Mestre em Engenharia 
José Augusto Calvo Lonardoni

\section{Desenvolvimento de um Simulador da Mecânica Cardiovascular Humana controlada pelo Mecanismo Reflexo Baroceptor}

Dissertação apresentada à Escola Politécnica da Universidade de São Paulo para obtenção do título de Mestre em Engenharia

Área de Concentração:

Engenharia Mecatrônica

Orientador:

Prof. Doutor Agenor de Toledo Fleury 
Este exemplar foi revisado e alterado em relação à versão original, sob responsabilidade única do autor e com a anuência de seu orientador.

São Paulo, 14 de outubro de 2006.

Assinatura do autor

Assinatura do orientador

FICHA CATALOGRÁFICA

Lonardoni, José Augusto Calvo

Desenvolvimento de um simulador da mecânica cardiovascu-

lar humana controlada pelo mecanismo reflexo baroceptor $I$

J.A.C. Lonardoni. - ed.rev. - São Paulo, 2006.

$143 \mathrm{p}$.

Dissertação (Mestrado) - Escola Politécnica da Universidade de São Paulo. Departamento de Engenharia Mecatrônica e de Sistemas Mecânicos.

1.Modelagem matemática 2.Simulação (Aprendizagem) 3.Bioengenharia 4.Sistema cardiovascular 5.Softwares (Simulação computacional) I.Universidade de São Paulo. Escola Politécnica. Departamento de Engenharia Mecatrônica e de Sistemas Mecânicos II.t. 
Aos meus pais, Silvio e Maria, que sempre me motivaram a acreditar em meus sonhos que sempre me ensinaram o valor da educação, e que sempre me suportaram e ampararam, de todas as formas imagináveis. 
Se você só tiver um martelo, tenderá a ver todo problema como um prego. Abraham Maslow 


\section{Agradecimentos}

Ao orientador e amigo Prof. Dr. Agenor de Toledo Fleury,

Pela confiança depositada em meu potencial e pelas inestimáveis experiências transmitidas em nossas conversas.

Ao Dr. José Otávio Costa Auler Júnior,

Pelas orientações na área médica, tão necessárias a este trabalho.

À Intermed Equipamento Médico Hospitalar Ltda. (principalmente ao Dr. Jorge Bonassa), Por possibilitar o desenvolvimento deste trabalho e por mostrar que vale a pena investir no desenvolvimento de produtos e competências nacionais.

Ao Prof. Dr. Raul Gonzalez Lima, ao Prof. Dr. José Augusto Lopes e ao Prof. Dr. Aron José Pazin de Andrade,

Pelas valiosas orientações por ocasião do exame de qualificação e da banca de dissertação.

\section{Ao Prof. Dr. Lucas Antônio Moscato,}

Pelas primeiras orientações de iniciação científica e por algumas dos primeiros ensinamentos recebidos na engenharia.

\section{À minha irmã Andréa,}

Por todo o apoio, afeto e companheirismo demonstrados ao longo de minha vida, seja a 0.001 , a 700 ou a mais de $1000 \mathrm{~km}$ de distância. E por todos os sábios conselhos e puxões de orelha que só uma irmã maior tem o direito de dar.

\section{À minha namorada Tatiani,}

Por toda a compreensão, apoio e amor (além de sua enorme paciência), que tornaram tudo mais fácil, não apenas neste trabalho. Além, é claro, das valiosíssimas idéias e opiniões, sempre colocadas naqueles inevitáveis momentos de desespero.

\section{Aos amigos da Poli e da Intermed,}

Pelo convívio de amizade e descontração criado nestes últimos tempos, que em muito contribuiu (ou não) para o desenvolvimento deste trabalho. 


\section{Resumo}

Nos últimos anos, o ensino de fisiologia tem sido fortemente beneficiado pelo desenvolvimento de modelos matemáticos e simuladores de paciente capazes de reproduzir com segurança partes específicas ou sistemas fisiológicos completos. Estudos mostram que aulas teóricas aliadas a simulações conseguem potencializar o nível de compreensão dos conceitos envolvidos. O principal objetivo deste trabalho consiste no desenvolvimento de um simulador do sistema cardiovascular, capaz de representar de forma didática a dinâmica do ciclo cardíaco. A disponibilidade de simuladores deste tipo é reduzida, e limitada a modelos simplificados ou interfaces pouco amigáveis. De modo a conseguir maior flexibilidade nas situações simuladas e um adequado grau de proximidade com o sistema real, decidiu-se pela utilização de um modelo matemático da fisiologia cardiovascular para o cálculo das variáveis e parâmetros atuantes no plano de fundo do simulador. O desenvolvimento deste modelo constitui o segundo objetivo deste trabalho. Devido ao grau de complexidade desejado, optou-se pela utilização de um modelo existente na literatura, acrescido dos parâmetros que fossem considerados ausentes. $O$ resultado foi um modelo com oito compartimentos vasculares e quatro compartimentos representando as câmaras do coração, todas com atividade pulsátil, controlados pelo mecanismo reflexo baroceptor, controle de curto prazo que atua na regulação da pressão arterial. O simulador (desenvolvido em Visual C\# com interface em Macromedia Flash) permite a alteração individual dos parâmetros vasculares e a simulação de hemorragias, bem como a visualização de gráficos de pressão, fluxo e volume em qualquer compartimento, e a construção de alças pressão-volume para os átrios e ventrículos. Além disso, o aplicativo resultante possibilita a inclusão futura de novos recursos e ferramentas, como tutoriais e simulação de patologias. 


\section{Abstract}

During the last years, physiology learning has been strongly favored by the development of mathematical models and patient simulators capable of safely reproduce specific parts or complete physiological systems. Studies show that traditional classes together with simulations are able to increase the comprehension of concepts involved. The main objective of this work is to develop a simulator of the cardiovascular system capable of representing the cardiac cycle dynamics in a didactic fashion. There are just a few available simulators of this kind, and the existing ones are based on simplified models or unfriendly interfaces. In order to achieve high flexibility in simulated scenarios and an adequate level of realism, we decided to use a mathematical model of the cardiovascular physiology to calculate variables and parameters acting in the background of the application. The development of this model constitutes the second objective of the present work. Due to the desired level of complexity, we decided to use an existing model found in the literature, improved with additional parameters we found necessary. The result is a model with eight vascular compartments and all four cardiac chambers, with pulsatile behavior, controlled by the baroreflex mechanism, a short term control that regulates arterial pressure. The simulator (developed in Visual C\# with an interface built in Macromedia Flash) allows the user to change individual parameters and simulate blood losses, as well as visualize press, flow and volume graphs from any compartment and also pressure-volume loops from the cardiac chambers. Moreover, the resulting application is open to future inclusion of new resources and tools, such as tutorials and pathology simulation. 


\section{Sumário}

\section{Resumo}

Abstract

Sumário

Lista de Figuras

Lista de Tabelas

Lista de Abreviaturas e Siglas

Lista de Símbolos

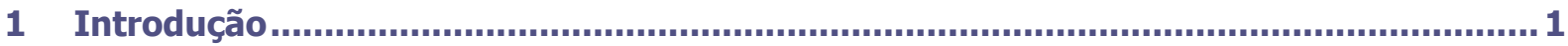

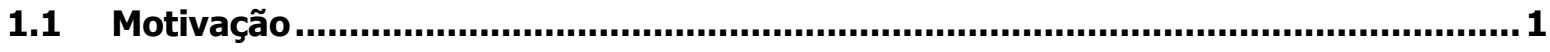

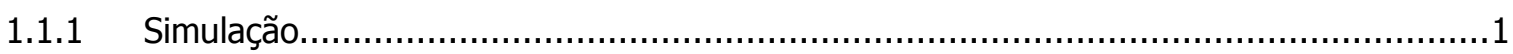

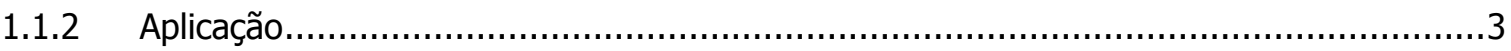

1.2 Objetivos .............................................................................................. 4

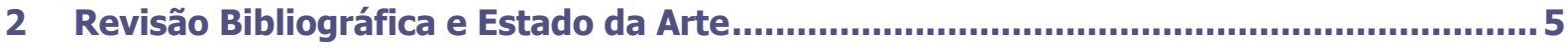

2.1 Modelagem do Sistema Cardiovascular a Parâmetros Concentrados..................... 5

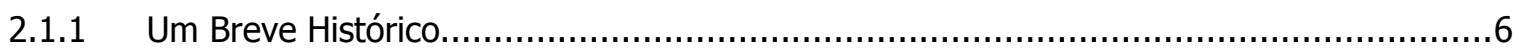

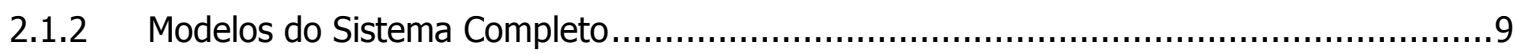

2.1.3 Modelos Multi-Escalares................................................................................. 13

2.2 Modelagem dos Mecanismos de Controle e Regulação ....................................... 14

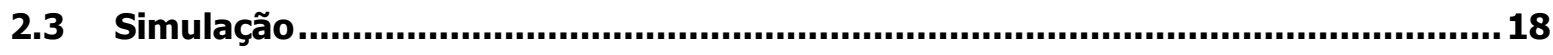

2.3.1 Simuladores de Tela (screen-based) e Simuladores de Escala Natural (full-scale)..........19

2.3.2 Simulação Controlada por Roteiros e Simulação Baseada em Modelos..........................20

2.3.3 Simulação Didática do Sistema Cardiovascular ................................................. 21 
3 Modelagem e Simulação do Sistema Cardiovascular Controlado............................. 24

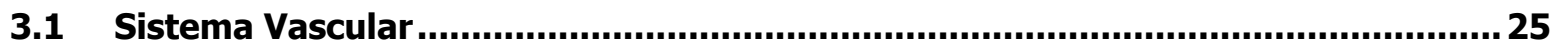

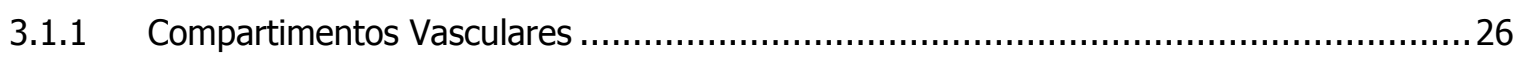

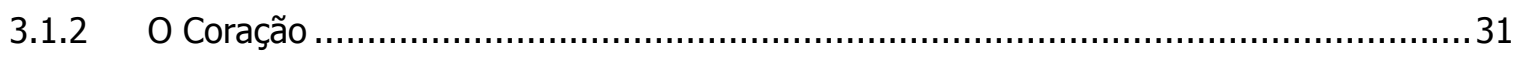

3.2 Sistema de Controle - O Mecanismo Barorreflexo............................................. 39

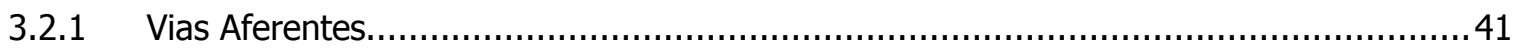

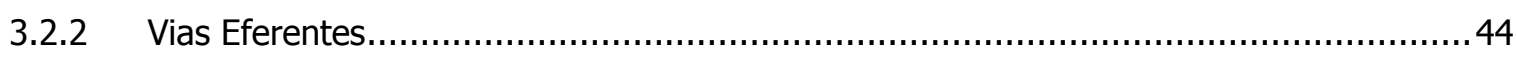

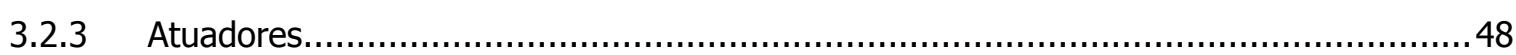

3.3 Modelagem dos Átrios Ativos.................................................................... 52

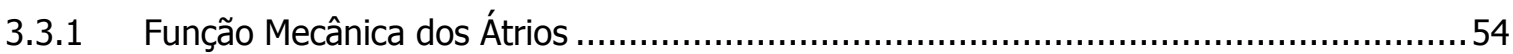

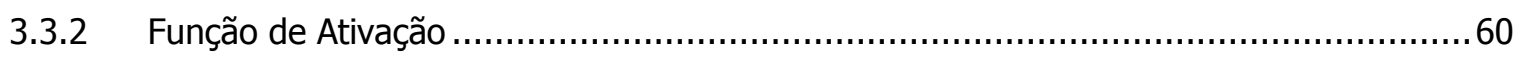

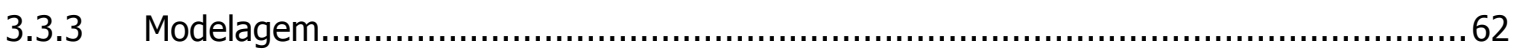

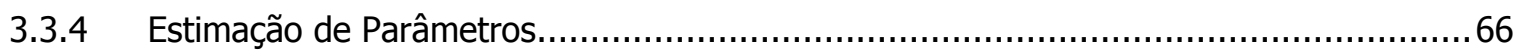

4 Resultados das Simulações Numéricas .................................................... 68

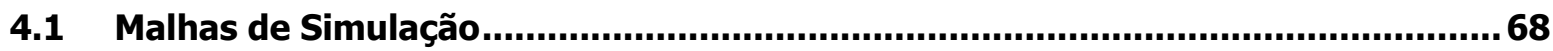

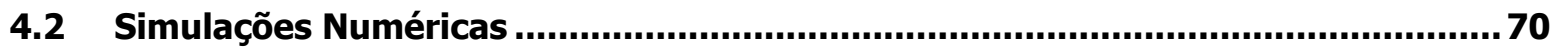

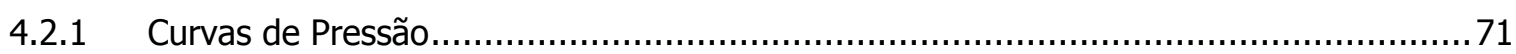

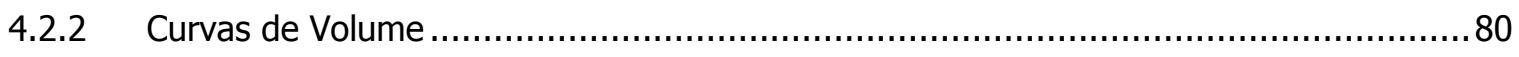

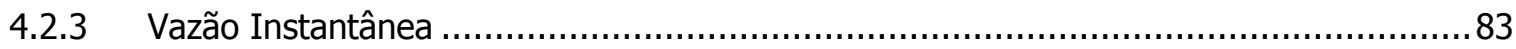

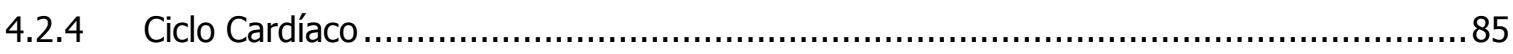

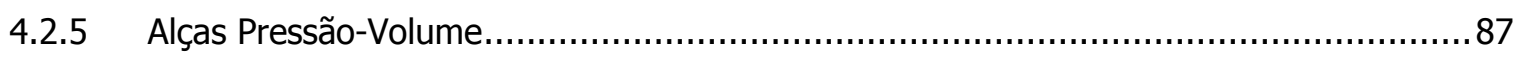

4.2.6 Resposta do Mecanismo Barorreflexo ..................................................................... 94

5 Desenvolvimento do Simulador Didático .................................................................... 96

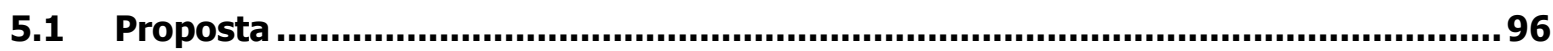

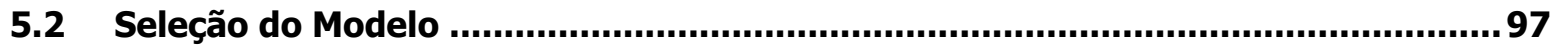

5.3 Discretização e Algoritmo de Integração........................................................... 98

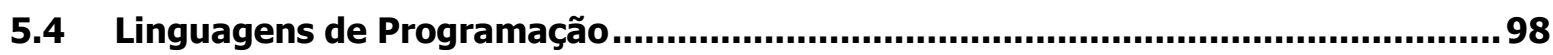

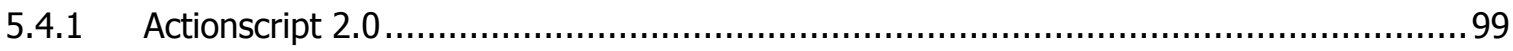

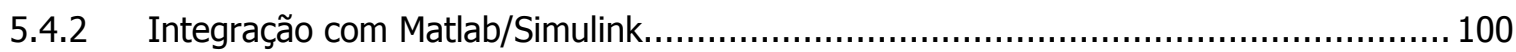

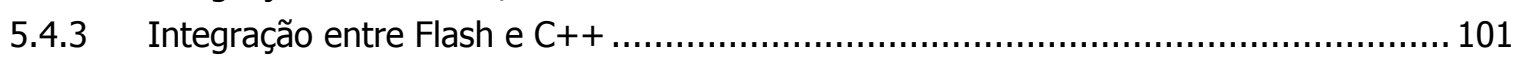

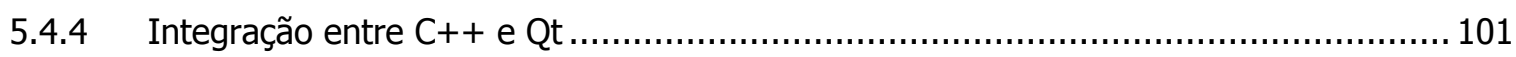

5.4.5 Integração entre Flash e C\# .......................................................................... 101

5.5 Interface e Arquitetura Funcional............................................................... 102 


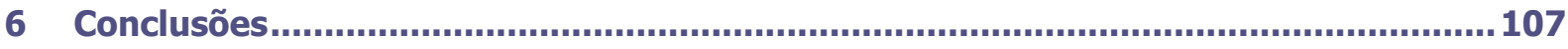

6.1 Sugestões para trabalhos futuros .................................................................. 109

7 Referências Bibliográficas .................................................................................... 110

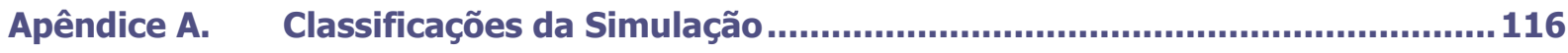

A.1 As 11 Dimensões da Simulação............................................................. 116

Apêndice B. Diagramas de Blocos do Modelo com Átrios Ativos ............................... 119 


\section{Lista de Figuras}

Figura 2-1 Modelo windkessel composto por dois elementos, um capacitivo e um resistivo (adaptado de FUNG, 1997).

Figura 2-2 Esquema do sistema cardiovascular humano, conforme proposto por Žáček e Krause

(1996).

Figura 2-3 Modelo do sistema cardiovascular sugerido por Beneken em 1965 e adaptado por Goodwin em 2004 para uso em simuladores pediátricos em escala natural (adaptado de Goodwin, 2004).

Figura 2-4 Resultados de pressão arterial sistêmica (à esquerda) e alças pressão-volume (à direita) para o ventrículo esquerdo de um paciente com e sem estenose aórtica (GOODWIN, 2004)......11

Figura 2-5 Analogia hidráulica do sistema cardiovascular proposta por Ursino em 1998. 12

Figura 2-6 Efeitos da atuação do barorreflexo (à esquerda) e da variação da freqüência cardíaca (à direita) obtidos por Ursino (1998).

Figura 2-7 Exemplos de modelos multi-escalares. (a) Modelo proposto por Formaggia et al. (1999), que utiliza as equações de Navier-Stokes para resolver o escoamento do sangue de forma mais precisa. (b) Modelo proposto por Laganà et al. (2004), que utiliza um modelo em elementos finitos da artéria pulmonar.

Figura 2-8 Interface principal do simulador CVI (ROTHE; GERSTING, 2002)

Figura 3-1 Modelo hidráulico do sistema cardiovascular, apresentando os elementos que compõem os compartimentos vasculares. No diagrama, procurou-se preservar certa associação anatômica nas posições dos elementos. As setas indicam o sentido do fluxo sangüíneo.

Figura 3-2 Modelo hidráulico mostrando as variáveis que o descrevem. As câmaras do coração são identificadas por índices em letras maiúsculas.

Figura 3-3 Relação pressão-volume do ventrículo esquerdo. A alça pressão-volume exibida em (b) é obtida a partir das variações de pressão e volume em um ciclo cardíaco, exibido em (a). As fases do ciclo estão numeradas em algarismos romanos: I. fase de enchimento, II. contração isovolumétrica, III. fase de ejeção, IV. relaxamento isovolúmico - note as fases isovolumétricas em (a) e (b), representadas pelo volume diastólico final (VDF) e volume sistólico final (VSF). As linhas tracejadas em (b) marcadas como $a$ (em vermelho) e $b$ (em azul) representam as funções pressão-volume na diástole e no fim da sístole, respectivamente (adaptado de Rhoades, 2003, com base em Ursino, 1998 e Guyton, 1997).

Figura 3-4 Função de ativação do ventrículo $\varphi(t)$.

Figura 3-5 Função de ativação do ventrículo $\varphi(t)$ e função de disparo $u(t)$. Note que o ciclo em $\varphi(t)$ inicia quando $\mathrm{u}(\mathrm{t})$ passa para zero.

Figura 3-6 Anatomia dos vasos que possuem os mais importantes barorreceptores (vista frontal). Vasos sangüíneos: a - arco aórtico, b - tronco pulmonar, c - artéria subclávica, d - artéria carótida comum, e - seio carotídeo, $\mathrm{f}$ - artéria carótida interna, $\mathrm{g}$ - artéria carótida externa. Vias nervosas: 1 - nervo glossofaríngeo, 2 - nervo do seio carotídeo, 3 - nervo vago, 4 - nervo laríngeo superior, 5 - ramos vagos cardíacos (originados no arco aórtico e suas ramificações), 6 nervo laríngeo recorrente, 7 - ramos vagos cardíacos (originados no tronco pulmonar e suas ramificações) (adaptado de Karemaker, 1987). 
Figura 3-7 Resposta do barorreceptor carotídeo em diferentes níveis de pressão arterial (adaptado de Guyton e Hall, 1996).

Figura 3-8 Modelo hidráulico do sistema cardiovascular com os elementos componentes do controle pelo mecanismo barorreflexo, com os caminhos nervosos mostrados através das linhas pontilhadas.

Figura 3-9 Resposta simulada do barorreceptor carotídeo.

Figura 3-10 Efeitos do aumento da pressão arterial sobre as atividades simpática e parassimpática, resultando na alteração da freqüência cardíaca, da força de contração e da resistência vascular sistêmica (adaptado de Rhoades e Tanner, 2003).

Figura 3-11 Função de atividade eferente simpática. .46

Figura 3-12 Função de atividade eferente parassimpática (vaga). 46

Figura 3-13 Ilustração das ondas $a, c$ e $v$, para os lados direito e esquerdo do coração (adaptado de Antoni, 1996b).

Figura 3-14 Simulação do ciclo cardíaco no modelo com átrios passivos, destacando as curvas de

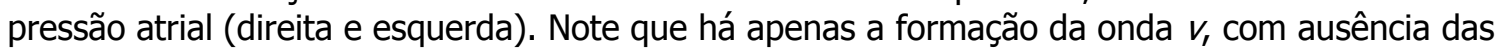
ondas $a$ e $c$ (as letras entre parênteses indicam as posições onde estas ondas deveriam aparecer). Além disso, os valores estão mais baixos que o esperado (entre 10 e $15 \mathrm{mmHg}$ )......53

Figura 3-15 Curvas de pressão e volume no átrio esquerdo, conforme mostrado por diferentes trabalhos. Os traçados de pressão e volume e a alça em (a) foram adaptados de Pagel et al. (2003), que utilizaram ecocardiografias com Doppler para estimação dos valores. Os traçados em (b) foram adaptados de Matsuda et al. (1983), que utilizaram angiocardiografias auxiliadas por cateteres com micro-manômetros. FVM significa o fechamento da válvula mitral, representado nas alças pelo pequeno círculo preto à esquerda do traçado, e $A V M$ significa a abertura da mesma válvula. Nas alças pressão-volume, é representado um ciclo cardíaco, iniciado no fechamento da válvula mitral. As denominações das regiões dos gráficos são as encontradas na literatura $(a, c e$ $v$ nos traçados de pressão e $A$ e $V$ nas alças).

Figura 3-16 Curvas de pressão e volume no átrio direito, conforme mostrado por diferentes trabalhos. Os gráficos em (a) foram adaptados de Maniar et al. (2003), que utilizaram cateteres especificamente construídos para a estimação das pressões e volumes através da medição de condutância em ovelhas. Os traçados em (b) foram adaptados de Miller et al. (1986), que utilizaram cateteres de impedância para estimação dos volumes e micro-manômetros para medição de pressão em porcos. Note que este trabalho utiliza unidades relativas de volume, pois o método é incapaz de fornecer uma estimativa dos valores absolutos. FVT significa o fechamento da válvula tricúspide, representado nas alças pelo pequeno círculo preto à esquerda do traçado, $\mathrm{e}$ AVT significa a abertura da mesma válvula. Nas alças pressão-volume, é representado um ciclo cardíaco, iniciado no fechamento da válvula tricúspide.

Figura 3-17 Determinação das elastâncias para o átrio esquerdo. EMAX é a elastância máxima, obtida pela inclinação da reta que liga os pontos correspondentes ao fim da sístole atrial. EMIN é a elastância mínima, determinada através da inclinação da reta obtida pela união dos pontos correspondentes ao final da diástole atrial (adaptado de Pagel et al., 2003).

Figura 3-18 Determinação das elastâncias do átrio direito. (adaptado de Maniar et al., 2003). ....59

Figura 3-19 Propagação do estímulo elétrico pelo músculo cardíaco (a seta vermelha indica o sentido de condução). O impulso rítmico normal é gerado no Nodo SA, ou sinoatrial (ou ainda sinusal) e conduzido até o Nodo AV, ou Atrioventricular, sofrendo um retardo de $160 \mathrm{~ms}$. $\mathrm{O}$ impulso continua pelo Feixe AV e é conduzido para os feixes esquerdo e direito das fibras de Purkinje, que distribuem o impulso por todas as partes dos ventrículos. (Adaptado de Rhoades e Tanner, 2003 e 3DScience.com, com base em Guyton e Hall, 1997). 
Figura 3-20 Diferenças na duração da contração dos átrios e dos ventrículos. Antoni (1996) mostra as durações dos potenciais de ação em um coelho. (Adaptado de Antoni, 1996a).

Figura 3-21 Temporização dos eventos do ciclo cardíaco. A sístole atrial é iniciada no final da diástole ventricular. A diástole atrial ocorre durante a sístole ventricular, permanecendo os átrios relaxados durante o restante do ciclo. As durações mostradas são relativas a uma freqüência cardíaca de 75 batimentos por minuto. (Adaptado de Fox, 2006).

Figura 3-22 Função de ativação dos átrios (azul) e dos ventrículos (tracejado verde).

Figura 3-23 Função de ativação dos átrios $\varphi_{\mathrm{a}}(\mathrm{t})$, em azul, e dos ventrículos $\varphi_{\mathrm{v}}(\mathrm{t})$, em tracejado verde. Note que a fração do ciclo cardíaco $\mathrm{u}(\mathrm{t})$ é a mesma para ambas.

Figura 4-1 Malha de simulação em Simulink para o modelo com átrios ativos.

Figura 4-2 Malha de simulação em Simulink para o modelo com átrios passivos.

Figura 4-3 Simulação do modelo com átrios passivos. O gráfico da esquerda mostra os resultados da simulação em Matlab do modelo apresentado na Seção 3.1, com os parâmetros extraídos do trabalho de Ursino (1998). O gráfico da direita foi extraído diretamente do trabalho citado.........71

Figura 4-4 Comparação da pressão arterial sistêmica do modelo com átrios passivos e do paciente 055 do MIMIC.

Figura 4-5 Comparação da simulação da pressão arterial sistêmica dos modelos com átrios passivos e com átrios ativos.

Figura 4-6 Pressões no ventrículo esquerdo e nas artérias sistêmicas para o modelo com átrios ativos, com valor de inércia aumentado (para $0.001 \mathrm{mmHg} \cdot \mathrm{ml} / \mathrm{s}^{2}$ )......

Figura 4-7 Comparação entre a pressão pulmonar arterial simulada com o modelo com átrios ativos e o banco de dados MIMIC 231/231. Foram mantidos os símbolos originais, tanto do modelo, na figura da esquerda, quanto do paciente (símbolo usual no meio médico), apesar de ambos representarem a mesma pressão.

Figura 4-8 Exemplo de alteração de parâmetros inadequada, provocando deformações na pressão ventricular direita. Os parâmetros do gráfico da esquerda são os mesmos sugeridos por Ursino (com exceção da indutância), ou seja, $C_{p a}=0.76 \mathrm{ml} / \mathrm{mmHg}, R_{p a}=0.023 \mathrm{mmHg} \cdot \mathrm{s} / \mathrm{ml} \mathrm{e} \mathrm{L}_{\mathrm{pa}}=$ $0.0007 \mathrm{mmHg} \cdot \mathrm{ml} / \mathrm{s}^{2}$. No gráfico da direita foram utilizados $C_{p a}=1.76 \mathrm{ml} / \mathrm{mmHg}, R_{p a}=0.45$ $\mathrm{mmHg} \cdot \mathrm{s} / \mathrm{ml} \mathrm{e} \mathrm{L}_{\mathrm{pa}}=0.0018 \mathrm{mmHg} \cdot \mathrm{ml} / \mathrm{s}^{2}$.

Figura 4-9 Comparação entre a pressão venosa central simulada com o modelo com átrios ativos e o banco de dados MIMIC 474/474.

Figura 4-10 Comparação entre a pressão atrial esquerda no modelo com átrios ativos (à esquerda) e com átrios passivos (à direita).

Figura 4-11 Traçados experimentais de pressão atrial esquerda, adaptados, respectivamente de cima para baixo, de Matsuda et al. (1983) e de Pagel et al. (2003).

Figura 4-12 Comparação entre a pressão atrial direita no modelo com átrios ativos (à esquerda) e com átrios passivos (à direita).

Figura 4-13 Traçados experimentais de pressão no átrio direito, adaptados, respectivamente de cima para baixo, de Miller et al. (1986) e de Maniar et al. (2003).

Figura 4-14 Comparação entre os traçados de pressão no ventrículo esquerdo resultantes da simulação do modelo com átrios ativos (à esquerda) e com átrios passivos (à direita).

Figura 4-15 Curva de pressão ventricular esquerda adaptada de Guyton e Hall (1996). O período do ciclo foi estimado entre 0.8 e $1 \mathrm{~s}$, a partir de comentários dos autores. 
Figura 4-16 Ampliação da região em que aparece a elevação causada pela onda $a$ da pressão atrial

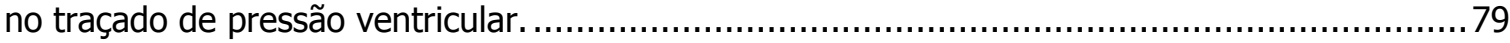

Figura 4-17 Comparação entre a pressão no ventrículo direito originada da simulação do modelo com átrios ativos (à esquerda) e passivos (à direita). ...................................................... 79

Figura 4-18 Pressões no lado direito do coração. A linha azul representa a pressão no ventrículo direito. Podem-se notar, através da linha pontilhada, as ondas $a, c$ e $v$ da pressão atrial, e a influência da primeira delas na pressão ventricular (adaptado de Antoni, 1996b).

Figura 4-19 Comparação entre o volume atrial esquerdo no modelo com átrios ativos (à esquerda) e com átrios passivos (à direita).

Figura 4-20 Traçados experimentais de volume atrial esquerdo. 0 traçado superior foi adaptado de Matsuda et al. (1983), e o inferior, de Pagel et al. (2003).

Figura 4-21 Comparação entre os resultados de volume atrial direito calculados a partir do modelo com atividade atrial (à esquerda) e do modelo sem atividade atrial (à direita).

Figura 4-22 Curvas de volume no átrio direito, obtidas experimentalmente por Maniar et al. (2003) e por Miller et al. (1986).

Figura 4-23 Volume no ventrículo esquerdo após (gráfico à esquerda) e antes (gráfico á direita) da inclusão da contratilidade atrial.

Figura 4-24 Curva de volume ventricular esquerdo adaptada de Guyton e Hall (1996)......

Figura 4-25 Curvas de volume no ventrículo direito, antes (gráfico da direita) e depois (gráfico da esquerda) da inclusão dos átrios ativos.

Figura 4-26 O gráfico da esquerda mostra a curva de débito cardíaco resultante da simulação em Matlab do modelo com átrios passivos, utilizando os mesmos parâmetros sugeridos no trabalho de Ursino (1998). O gráfico da direita foi obtido diretamente do trabalho mencionado.

Figura 4-27 Simulação do débito cardíaco do ventrículo esquerdo do modelo com átrios ativos, em comparação com o mesmo gráfico extraído de Ursino (1998), mostrado na Figura 4-26 acima... 84

Figura 4-28 Comparação das curvas de débito cardíaco do lado direito do coração após (gráfico da esquerda) e antes (gráfico da direita) da inclusão da contratilidade dos átrios.

Figura 4-29 Ciclo cardíaco, mostrando as pressões aórtica, atrial e ventricular e o volume ventricular do lado esquerdo, bem como o eletro e o fonocardiograma (GUYTON e HALL, 1996). Assim como na Figura 4-15, estima-se o período cardíaco do gráfico entre 0.8 e $1 \mathrm{~s} . \ldots \ldots \ldots \ldots \ldots . . . . . . .85$

Figura 4-30 Simulação do ciclo cardíaco para o modelo com átrios ativos. ................................86

Figura 4-31 Simulação do ciclo cardíaco para o modelo com átrios passivos. …......................... 87

Figura 4-32 Determinação da alça pressão-volume do ventrículo esquerdo (adaptado de Antoni,

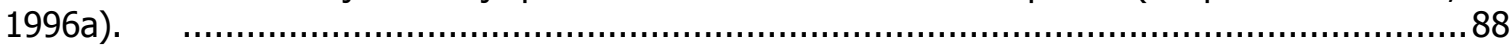

Figura 4-33 Alça pressão-volume do ventrículo esquerdo (RHOADES e TANNER, 2003). .............88

Figura 4-34 Alça pressão-volume calculada para o ventrículo esquerdo (modelo com átrios ativos).

Figura 4-35 Alça pressão-volume calculada para o ventrículo esquerdo (modelo com átrios passivos).

Figura 4-36 Alça pressão volume calculada para o ventrículo direito (modelo com átrios ativos). . 90

Figura 4-37 Alça pressão volume calculada para o ventrículo direito (modelo com átrios passivos). 
Figura 4-38 Alças pressão-volume experimentais determinadas para o átrio esquerdo, adaptadas de (a) Matsuda et al. (1983), e de (b) Pagel et al. (2003).................................................... 91

Figura 4-39 Alça pressão-volume calculada para o átrio esquerdo (modelo com átrios ativos).....91

Figura 4-40 Relação pressão-volume para o átrio esquerdo (modelo com átrios passivos)...........92

Figura 4-41 Alças pressão-volume experimentais determinadas para o átrio direito, adaptadas de

(a) Maniar et al. (2003), e de (b) Miller et al. (1986)............................................................92

Figura 4-42 Alça pressão-volume calculada para o átrio direito (modelo com átrios ativos).........93

Figura 4-43 Relação pressão-volume para o átrio direito (modelo com átrios passivos)...............93

Figura 4-44 Pressão no ventrículo esquerdo. A curva mais clara corresponde à pressão pulsátil, efetiva no ventrículo, e a linha mais escura representa apenas o envelope da curva, para maior clareza.

Figura 4-45 Simulação de perda de um volume de sangue de $500 \mathrm{ml}$ ( $10 \%$ do volume total considerado). Novamente, a curva mais clara corresponde à pressão pulsátil e a linha mais escura representa o envelope da curva.

Figura 5-1 Simulador do sistema respiratório desenvolvido em trabalho anterior (LONARDONI e IWATA, 2002).

Figura 5-2 Simulador preliminar desenvolvido apenas em Flash e Actionscript 2.0 ................... 100

Figura 5-3 Interface do simulador no modo básico de operação. ............................................. 103

Figura 5-4 Janela de plotagem do modo básico, onde pode-se observar as três curvas (pressão,

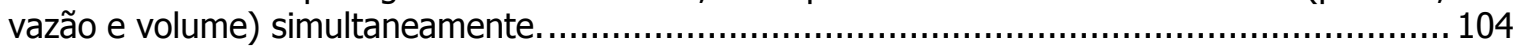

Figura 5-5 Interface do simulador no modo avançado de operação. ....................................... 104

Figura 5-6 Exemplos de janelas de configuração de parâmetros, para artérias pulmonares (figura à esquerda) e para a periferia esplâncnica (figura à direita) ................................................... 105

Figura 5-7 Janela de plotagem da curva de pressão ventricular esquerda................................. 106

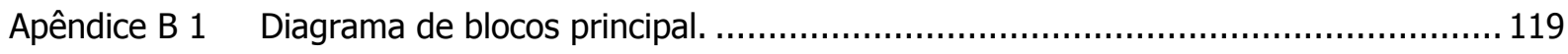

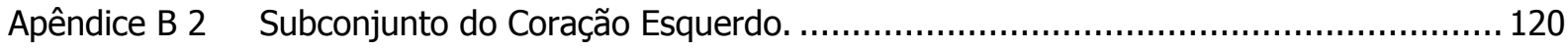

Apêndice B 3 Subconjunto do Coração Direito .................................................................. 121

Apêndice B 4 Subconjunto da Circulação Sistêmica ............................................................ 122

Apêndice B 5 Subconjunto da Circulação Pulmonar................................................................ 123

Apêndice B 6 Subconjunto do Barorreflexo Carotídeo........................................................ 123

Apêndice B 7 Subconjunto para determinação do Período Cardíaco. .......................................124

Apêndice B 8 Subconjunto da função de ativação. .............................................................. 124 


\section{Lista de Tabelas}

Tabela 3-1 Parâmetros referentes à circulação vascular. ........................................................... 30

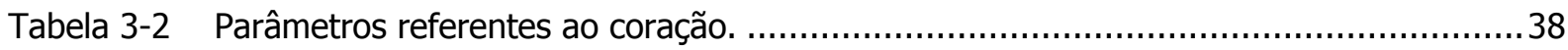

Tabela 3-3 Parâmetros que caracterizam a resposta produzida no seio carotídeo. .......................43

Tabela 3-4 Parâmetros relacionados à descrição dos impulsos eferentes. ..................................47

Tabela 3-5 Parâmetros referentes aos atuadores............................................................... 52

Tabela 3-6 Parâmetros estimados para o modelo com átrios ativos........................................67 


\section{Lista de Abreviaturas e Siglas}

$\begin{array}{ll}\text { AD } & \text { Átrio Direito } \\ \text { VD } & \text { Ventrículo Direito } \\ \text { AE } & \text { Átrio Esquerdo } \\ \text { VE } & \text { Ventrículo Esquerdo } \\ \text { VSF } & \text { Volume Sistólico Final } \\ \text { VDF } & \text { Volume Diastólico Final } \\ \text { FC, HR } & \text { Freqüência cardíaca (heart rate) } \\ \text { SA } & \text { Sinoatrial } \\ \text { AV } & \text { Atrioventricular } \\ \text { P-V } & \text { Pressão-Volume (referência às alças) } \\ \text { ABP } & \text { Pressão Sangüínea Arterial (Arterial Blood Pressure) } \\ \text { PAP } & \text { Pressão Pulmonar Arterial (Pulmonary Artery Pressure) } \\ \text { CVP } & \text { Pressão Venosa Central (Central Venous Pressure) } \\ \text { SNC } & \text { Sistema Nervoso Central } \\ \text { ECG } & \text { Eletrocardiograma } \\ \text { RIA } & \text { Rich Internet Applications }\end{array}$




\section{Lista de Símbolos}

\begin{tabular}{|c|c|}
\hline $\mathbf{P}$ & Pressão \\
\hline $\mathbf{v}$ & Volume \\
\hline $\mathbf{v}_{\mathbf{u}}$ & Volume não distendido (unstressed) \\
\hline$\dot{\mathbf{Q}}, \mathbf{F}$ & Fluxo ou vazão \\
\hline $\mathbf{R}$ & Resistência hidráulica \\
\hline C & Complacência \\
\hline $\mathbf{L}$ & Inertância hidráulica \\
\hline $\mathbf{T}$ & Período cardíaco \\
\hline $\mathbf{T}_{\text {sis }}$ & Período sistólico \\
\hline pa & Índice referente ao compartimento pulmonar arterial \\
\hline pp & Índice referente ao compartimento pulmonar periférico \\
\hline pv & Índice referente ao compartimento pulmonar venoso \\
\hline sa & Índice referente ao compartimento sistêmico arterial \\
\hline sp & Índice referente ao compartimento esplâncnico periférico \\
\hline ep & Índice referente ao compartimento extra-esplâncnico periférico \\
\hline sv & Índice referente ao compartimento esplâncnico venoso \\
\hline ev & Índice referente ao compartimento extra-esplâncnico venoso \\
\hline AD & Índice referente ao compartimento atrial direito \\
\hline VD & Índice referente ao compartimento ventricular direito \\
\hline $\mathbf{A E}$ & Índice referente ao compartimento atrial esquerdo \\
\hline VE & Índice referente ao compartimento ventricular esquerdo \\
\hline$\dot{\mathbf{Q}}_{\text {dir }, \mathbf{e}}$ & Fluxo de saída do átrio (e de entrada do ventrículo) direito \\
\hline$\dot{\mathbf{Q}}_{\mathrm{dir}, \mathbf{s}}$ & Fluxo de saída do ventrículo direito \\
\hline$\dot{\mathbf{Q}}_{\text {esq,e }}$ & Fluxo de saída do átrio (e de entrada do ventrículo) esquerdo \\
\hline$\dot{\mathbf{Q}}_{\text {esq,s }}$ & Fluxo de saída do ventrículo esquerdo \\
\hline $\mathbf{V}_{\mathbf{t}}$ & Volume total de sangue \\
\hline $\mathbf{I}(\mathbf{t})$ & Taxa de injeção ou retirada de sangue \\
\hline $\mathbf{d} / \mathbf{d t}$ & Derivada em relação ao tempo \\
\hline$\varphi(\mathbf{t})$ & Função de ativação dos átrios e ventrículos \\
\hline
\end{tabular}




$\begin{array}{ll}\mathbf{u}(\mathbf{t}) & \text { Adimensional relacionado à função de ativação } \\ \mathbf{E}_{\max } & \text { Elasticidade sistólica final } \\ \mathbf{P}_{\max } & \text { Pressão isométrica } \\ \mathbf{k}_{\mathbf{E}} & \text { Constante de elasticidade } \\ \mathbf{k}_{\text {sis }} & \text { Constante relacionada ao período sistólico } \\ \mathbf{f} & \text { Freqüência de impulsos aferentes e eferentes } \\ \tau & \text { Constante de tempo } \\ \mathbf{p} & \text { Índice referente a pólo } \\ \mathbf{z} & \text { Índice referente a zero } \\ \mathbf{s c} & \text { Índice referente ao seio carotídeo } \\ \mathbf{e s} & \text { Índice referente aos impulsos eferentes simpáticos } \\ \mathbf{e v} & \text { Índice referente aos impulsos eferentes vagos (parassimpáticos) } \\ \mathbf{G} & \text { Ganhos dos atuadores } \\ \mathbf{D} & \text { Atrasos dos atuadores }\end{array}$




\section{Introdução}

Nos últimos anos o sistema cardiovascular humano tem sido alvo da atenção de uma grande comunidade de pesquisadores, não apenas da área médica, como seria natural esperar, mas também das áreas de engenharia, matemática e computação. Uma revisão bibliográfica sobre o assunto revela que uma infinidade de trabalhos vem sendo publicada, além de existirem, já há algum tempo, publicações especializadas na denominada engenharia cardiovascular. Evidentemente, em uma área caracterizada por tamanha multidisciplinaridade, há também uma enorme variedade no nível de abrangência das pesquisas, envolvendo tanto o estudo de sistemas isolados (como a modelagem da função do ventrículo esquerdo ou da válvula aórtica, ou ainda a modelagem do escoamento sangüíneo na microcirculação) quanto do sistema como um todo, incluindo mecanismos de controle e auto-regulação.

Juntamente com o desenvolvimento dos modelos matemáticos que regem o comportamento do sistema e suas partes individuais, cresceu também o interesse pela simulação, agora mais voltada para o organismo com um todo. Este tipo de simulação surgiu mais especificamente da anestesiologia, em que a prática e o treinamento são fundamentais. Neste caso, a interface já não é mais numérica, e nem mesmo na tela do computador, mas sim em forma de bonecos em tamanho natural, que reagem de diversas formas aos procedimentos realizados. Conseqüentemente, há a necessidade de agregação de uma maior quantidade e variedade de sistemas fisiológicos e fenômenos de outras naturezas, de modo a prover um maior grau de realismo e envolvimento dos treinados.

\subsection{Motivação}

\subsubsection{Simulação}

A simulação é uma técnica utilizada para amplificar ou substituir experimentos reais por experimentos dirigidos que evoquem ou repliquem aspectos substanciais do mundo real de uma maneira completamente interativa. Nos últimos anos, tem crescido significativamente o interesse pelo uso da simulação com o propósito de melhorar a segurança dos pacientes e a qualidade dos tratamentos de 
saúde oferecidos. Essas aplicações podem envolver tanto ambientes imersivos, que induzam o usuário a se sentir dentro da situação simulada, agindo como se estivesse em seu real ambiente de trabalho, quanto ambientes normalmente não observáveis nas práticas comuns (tipicamente por impossibilidade física), mas que de alguma forma modifiquem sua observação ou permitam que os fenômenos que as originam sejam analisados.

A idéia que geralmente se tem de um simulador corresponde ao primeiro caso descrito acima, relacionado com dispositivos de imersão. Diversos exemplos destes equipamentos podem ser citados, como os simuladores de vôo (comerciais, militares ou espaciais), os simuladores de paciente em escala natural (para treinamento de procedimentos clínicos) e os simuladores de cirurgias (utilizando robôs cirúrgicos reais em pacientes simulados). Mais recentemente, o exército norte-americano vem utilizando simuladores até mesmo para treinar sua infantaria em combates corpo a corpo. Em todos estes casos, está envolvida a utilização de dispositivos que representem graficamente ou que utilizem os equipamentos alvo das simulações, de modo a proporcionar uma experiência de uso extremamente próxima da realidade.

Adicionalmente a estes simuladores mais popularmente conhecidos, existem também aplicações que se baseiam em modelos que representam a realidade física de algum sistema, sem haver a necessidade de fazer com que o usuário se sinta imerso naquela situação - até mesmo porque em muitos casos isso não faria sentido algum. Esse é o campo de uma particular categoria de simuladores fisiológicos, em que o principal interesse é proporcionar ao usuário (tipicamente um estudante) a possibilidade de observar e interagir em tempo real com um sistema biológico sem que se coloque o mesmo em situação de risco. Além disso, estas aplicações tornam viável a análise de parâmetros fisicamente impossíveis de serem mensurados, chegando-se até as causas e princípios que regem os fenômenos observados, e às origens e efeitos do desenvolvimento e evolução de certas patologias.

O uso de simulações para o ensino de fisiologia apresenta a grande vantagem de possibilitar a individualização de componentes fisiológicos de um sistema mais complexo, o que dificilmente poderia ser reproduzido na prática e in vivo em um laboratório, além de permitir a visualização gráfica e a mudança de parâmetros e variáveis, incluindo cálculo automático de variáveis derivadas e índices. Um simulador com certo nível de adaptabilidade ou inteligência artificial seria capaz de interagir com o 
usuário, mostrando a evolução (melhoria ou deterioração) das condições do paciente simulado. As simulações computadorizadas ainda evitam problemas éticos e riscos ou desconforto para o paciente, e permitem alta repetibilidade nos experimentos realizados, bem como o registro, análise e observação das técnicas utilizadas em uma simulação.

\subsubsection{Aplicação}

Pode-se notar que na literatura há uma carência de trabalhos sobre simuladores do segundo tipo mencionado acima - os simuladores didáticos, acessíveis a uma quantidade maior de pessoas e independentes de equipamentos ou locações especiais - aplicados ao ensino da fisiologia cardiovascular. É aí que se enquadra o objeto de trabalho desta dissertação.

Uma experiência anterior (LONARDONI e IWATA, 2002) mostrou a eficiência da utilização de simuladores didáticos no ensino de fundamentos fisiológicos. Aquele trabalho tratava do sistema respiratório, enfatizando a ventilação artificial. Através de um modelo simplificado e de uma interface limpa e intuitiva, foi possível transmitir alguns conceitos básicos, muito importantes para estudantes e profissionais da área de ventilação mecânica.

Michael (2001) publicou um trabalho (baseado em uma palestra de mesma autoria) que enfatizava as vantagens da simulação do sistema cardiovascular no ensino de fisiologia, principalmente nos primeiros anos de graduação. Seus experimentos relatavam o uso de simuladores bastante simples, mas capazes de desestimular o tradicional método de memorização em favor do raciocínio lógico, baseado em causas e efeitos. Desta forma, os alunos passavam a se preocupar mais com os princípios de funcionamento do sistema, ficando livres para a elaboração de soluções mais criativas e eficientes para os problemas propostos.

Mesmo assim, apesar da grande quantidade de trabalhos sobre modelagem do sistema cardiovascular, praticamente não há simuladores aplicados a ele, e os poucos existentes (ROTHE; GERSTING, 2002; CiRCSIM-Tutor) são baseados em modelos fisiológicos simplificados e focados na simulação de eventos estáticos. Os sistemas regulatórios, amplamente tratados em trabalhos de modelagem, quando abordados em aplicações de simulação dedicam-se apenas aos mecanismos de longo prazo. 


\subsection{Objetivos}

Motivado pelas considerações anteriores, propõe-se como principal objetivo deste trabalho, o desenvolvimento de um simulador do sistema cardiovascular capaz de representar de forma didática a dinâmica do ciclo cardíaco. O simulador deverá permitir a alteração de parâmetros com resposta imediata percebida através de indicadores visuais e parâmetros numéricos.

Para tanto, será necessário determinar os princípios básicos envolvidos no funcionamento do sistema cardiovascular, com a finalidade de se delimitar um conjunto mínimo de parâmetros que o representem. A partir destes parâmetros, deverá ser desenvolvido ou encontrado na literatura um modelo capaz de representar a dinâmica do sistema como um todo, incluindo seus aspectos hidrodinâmicos e auto-regulatórios. Com este modelo deverá ser possível simular o funcionamento do sistema cardiovascular controlado em situações de regime e de perda ou infusão de volume sangüíneo, de modo que seja possível observar a variação temporal de parâmetros como débito cardíaco e pressão, vazão e volume em vasos e compartimentos. Para este tipo de representação, o modelo deverá ter ordem elevada, onde nem todas as dinâmicas estarão acessíveis à medição direta, o que o torna não identificável a priori. Portanto, não será possível realizar para este modelo uma validação quantitativa, mas apenas uma verificação qualitativa com respeito à adequação de vários tipos de respostas simuladas em comparação com resultados conhecidos da literatura ou extraídos de bases de dados consolidadas.

De modo a tornar o simulador utilizável para qualquer pessoa, leiga ou não, o desenvolvimento do mesmo deverá seguir fundamentos de ergonomia cognitiva, que proporcionem a criação de uma interface amigável e intuitiva, que possa ser facilmente aprendida por qualquer usuário. Poderão ser utilizados também de conceitos de RIA (Rich Internet Applications), que se aproveitem da interatividade com recursos externos. 


\section{Revisão Bibliográfica e Estado da Arte}

Este capítulo procura apresentar alguns trabalhos relacionados aos tópicos de maior interesse a este projeto. Será traçado um panorama do estado da arte em modelagem e simulação do sistema cardiovascular humano, sendo apresentados os conceitos introduzidos em trabalhos que ajudaram a estabelecer as bases científicas do conhecimento sobre o assunto. Como muitos destes conceitos foram publicados há algumas décadas atrás, a maior parte dos originais não estava disponível, e serão citados de forma indireta por meio de artigos que os referenciaram.

O texto deste capítulo está organizado em três partes. A primeira parte trata da modelagem do sistema cardiovascular não controlado, apresentando um breve histórico da modelagem compartimental a parâmetros concentrados aplicada ao sistema em questão, desde o primordial modelo windkessel aos mais recentes estudos sobre a circulação e os órgãos. Em seguida, são descritos alguns modelos do sistema completo, com diferentes níveis de complexidade. A segunda parte trata da modelagem do sistema de controle da pressão arterial através do chamado reflexo baroceptor. Neste ponto, o leitor identificará que alguns dos modelos apresentados na primeira parte repetem-se na segunda. Isso acontece porque os estudos mais completos do sistema circulatório inevitavelmente necessitam de ambos os aspectos para permitirem uma melhor compreensão dos fenômenos envolvidos. Por fim, a terceira parte discute o desenvolvimento dos simuladores, diferenciando as variadas aplicações, mas direcionando uma maior ênfase às aplicações didáticas.

\subsection{Modelagem do Sistema Cardiovascular a Parâmetros Concentra- dos}

Como será possível notar ao longo deste capítulo, a predominância atual de publicações relacionadas ao funcionamento do sistema cardiovascular como um todo diz respeito à sua interdependência com os mecanismos de regulação. Isso se relaciona ao fato de as leis fundamentais que regem a circulação do sangue serem há mais tempo conhecidas, principalmente quando se considera um nível de abstração mais elevado. Neste caso, certos subsistemas podem ser vistos como caixas pretas, 
ou blocos mais simplificados, que desempenham funções bem determinadas. Estes elementos caracterizam a modelagem a parâmetros concentrados, compartimental, em que comumente é aplicada como método de resolução a analogia entre circuitos hidráulicos e elétricos. Neste âmbito, é possível ignorar os princípios individuais de cada componente, assumindo-se um comportamento aproximado que seja suficiente para representá-los (WHITE; FITZJERRELL; CROSTON, 1983). Como exemplo, pode-se mencionar a substituição dos efeitos viscosos da microcirculação por elementos resistivos.

Apesar destas aparentes simplificações e dos benefícios relacionados à compreensão do sistema como um todo, o elevado número de compartimentos utilizado nestes tipos de modelos corresponde a uma também elevada complexidade de resolução das equações associadas. Além disso, modelos a parâmetros concentrados geralmente consideram compartimentos não observáveis, cujos parâmetros não podem ser diretamente determinados (ou mesmo estimados), tornando-os nãoidentificáveis a priori. Por este motivo, validações quantitativas para estes casos tornam-se praticamente impossíveis, ou extremamente difíceis, sendo normalmente substituídas por verificações qualitativas, feitas com base em comparação com padrões conhecidos.

Existem ainda diversos aspectos e fenômenos individuais do sistema cardiovascular não completamente compreendidos, como o funcionamento isolado das câmaras e válvulas cardíacas, ou o escoamento do sangue nos diversos tipos de vasos. Estes itens continuam sendo alvos de uma enorme quantidade de publicações recentes.

\subsubsection{Um Breve Histórico}

O primeiro modelo de parâmetros concentrados utilizado no estudo do sistema cardiovascular foi sugerido por Stephen Hales $^{1}$ (1733 apud OLUFSEN; NADIM, 2004), e posteriormente desenvolvido por Otto Frank ${ }^{2}$ (1899 apud OLUFSEN; NADIM, 2004), com a finalidade de representar o fluxo e a pressão do sangue no sistema arterial. Este modelo, conhecido como windkessel, utiliza uma analogia entre o sistema coração e artéria com os antigos extintores operados manualmente pelos bombeiros

\footnotetext{
${ }^{1}$ HALES, S. Statical Essays: II Haemostatics. London: Innays and Manby, reimpresso por New York: Hafner, 1733.

2 FRANK, O. Die Grundform des arteriellen Pulses erste Abhandlung: mathematische Analyse. Z. Biol, v.37, p. 483-526, 1899.
} 
(denominada em alemão como bomba windkessel, que em uma tradução literal significa "reservatório de $\left.a r^{\prime \prime}\right)$. No caso do extintor, a água é bombeada pelo bombeiro para o interior de uma câmara de ar de alta pressão por meio de injeções periódicas a uma pressão maior. Quando o reservatório está cheio, a pressão média do reservatório faz com que a água armazenada seja expelida de maneira uniforme (FUNG, 1997). A analogia começa com o ventrículo esquerdo, onde a pressão do sangue varia de pouco mais de zero a até cerca de $120 \mathrm{mmHg}$ - caracterizando o funcionamento da bomba -, passando então para a aorta, cuja variação de pressão é significativamente menor - como o reservatório. O modelo windkessel original (de dois elementos) proposto por Frank afirmava que a aorta, assim como outras grandes artérias, poderia ser representada através de uma câmara elástica, enquanto os vasos periféricos eram substituídos por um tubo rígido de resistência constante ${ }^{3}$ (FUNG, 1997 e OLUFSEN; NADIM, 2004). Neste caso, o capacitor representa a complacência das grandes artérias, enquanto o resistor equivale à resistência das pequenas artérias e arteríolas, conforme Figura 2-1.

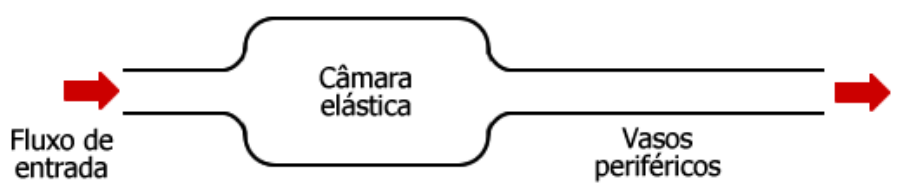

Figura 2-1 Modelo windkessel composto por dois elementos, um capacitivo e um resistivo (adaptado de FUNG, 1997).

O modelo windkessel de dois elementos consegue fornecer uma visão da contribuição das diferentes propriedades arteriais sobre a carga no coração, mas não representa adequadamente a impedância da aorta em médias e baixas freqüências (STERGIOPULOS; WESTERHOF, B. E; WESTERHOF, N., 1999). Posteriormente, o modelo de dois elementos foi expandido para três e para quatro elementos. O terceiro elemento consiste em um novo resistor, colocado em série com o circuito de dois elementos, de modo a representar a impedância característica da aorta e das grandes artérias (OLUFSEN; NADIM, 2004). O quarto elemento consiste em um indutor, representando a inércia do sangue (STERGIOPULOS; WESTERHOF, B. E; WESTERHOF, N., 1999). Todas estas expansões puderam incorrer em maior fidelidade à representação do comportamento do fluxo sangüíneo nas artérias.

\footnotetext{
${ }^{3}$ Na verdade, o modelo de Otto Frank foi originalmente derivado para o ventrículo e a aorta, e apenas posteriormente foi utilizado para descrever o fluxo sangüíneo nas artérias isoladas, sem a inclusão explícita do coração.
} 
Em 2003, Craiem e Armentano propuseram o conceito de complacência aparente, com a finalidade de unir ao modelo windkessel um modelo de linha de transmissão, capaz de contabilizar efeitos de propagação de ondas no sistema arterial.

$\mathrm{Na}$ tentativa de melhor compreender o sistema cardiovascular humano, diversas abordagens foram adotadas, resultando em uma seqüência de trabalhos que procuravam descrever aspectos individuais, com maior predominância para o sistema arterial. Em 1938, Hill ${ }^{4}$ (apud ŽÁČEK; KRAUSE, 1996) apresentou um modelo paramétrico da mecânica muscular do coração, seguido por modificações feitas por Grood et al. ${ }^{5}$ (1974 apud ŽÁČEK; KRAUSE, 1996) e por McLaughlin e Sonnenblick ${ }^{6}$ (1974 apud ŽÁČEK; KRAUSE, 1996) evoluindo sobre o comportamento elástico do músculo cardíaco. Em 1973, Suga et al." (apud GOODWIN et al., 2004; OOMEN et al., 2003; ŽÁČEK; KRAUSE, 1996) introduziram o modelo da elastância variável com o tempo para o ventrículo esquerdo. O conceito de elastância fora originalmente definido por Weber (1846 apud OOMEN et al., 2003) como sendo a razão entre a pressão e o volume ventriculares - o inverso da chamada complacência. O modelo de elastância variável introduzido por Suga et al. pode ser descrito como

$$
\mathbf{E}(\mathbf{t})=\frac{\mathbf{P}(\mathbf{t})}{\mathbf{V}(\mathbf{t})-\mathbf{V}_{\mathbf{u}}}
$$

onde $\mathbf{P}(\mathbf{t})$ e $\mathbf{V}(\mathbf{t})$ são respectivamente a pressão e o volume ventriculares e $\mathbf{V}_{\mathbf{u}}$ corresponde ao volume não distendido, no qual a pressão ventricular iguala a pressão externa ao ventrículo. Em 2003, este modelo foi aprofundado por Oomem et al., que demonstraram ser verdadeira a hipótese de que a elastância do ventrículo esquerdo poderia ser independente de carga, ou seja, independente das condições iniciais.

\footnotetext{
${ }^{4}$ HILL, A. V. Heat of shortening and the dynamic constants of muscle. Proceedings of the Royal Society of London: Biological Sciences, v. 126, p. 136-195, 1938.

${ }^{5}$ GROOD, E. S.; MATES, R. E.; FALSETTI, H. A model of cardiac muscle dynamics. Circulation Research, v. 35, p. 184-196, 1974.

${ }^{6}$ MCLAUGHLIN, R. J.; SONNENBLICK, E. H. Time behavior of series elasticity in cardiac muscle. Circulation Research, v. 34, p. 798-811, 1974.

7 SUGA, H.; SAGAWA, K.; SHOUKAS, A. A. Load independence of the instantaneous pressure-volume ratio of the canine left ventricle and effects of epinephrine and heart rate on the ratio. Circulation Research, v. 32, p. 314-322, 1973.
} 


\subsubsection{Modelos do Sistema Completo}

Vista a complexidade na descrição do sistema cardiovascular como um todo, apenas mais recentemente surgiram modelos associando os efeitos diversos estudados nos anos anteriores. Evoluindo nessa direção, Žáček e Krause (1996) propuseram, com base nos trabalhos de Jelinek ${ }^{8}$ (1972), Leaning et $a l .{ }^{9}{ }^{10}$ (1983), Avanzolini et al. ${ }^{11}$ (1988) e Stergiopulos et al. ${ }^{12}$ (1992), um modelo não controlado composto por 15 elementos (11 passivos e quatro ativos, correspondentes às câmaras do coração) que representam as principais partes do sistema (Figura 2-2) - todos os elementos seguem o modelo windkessel, sendo compostos por um reservatório elástico e um tubo rígido com resistência. Apesar de não controlado e de ignorar efeitos de inércia, o modelo é capaz de simular variações da função cardíaca (em decorrência de mudanças na freqüência cardíaca ou de anormalidades nas válvulas) e da pré- e pós-carga (conforme mecanismo de Frank-Starling).
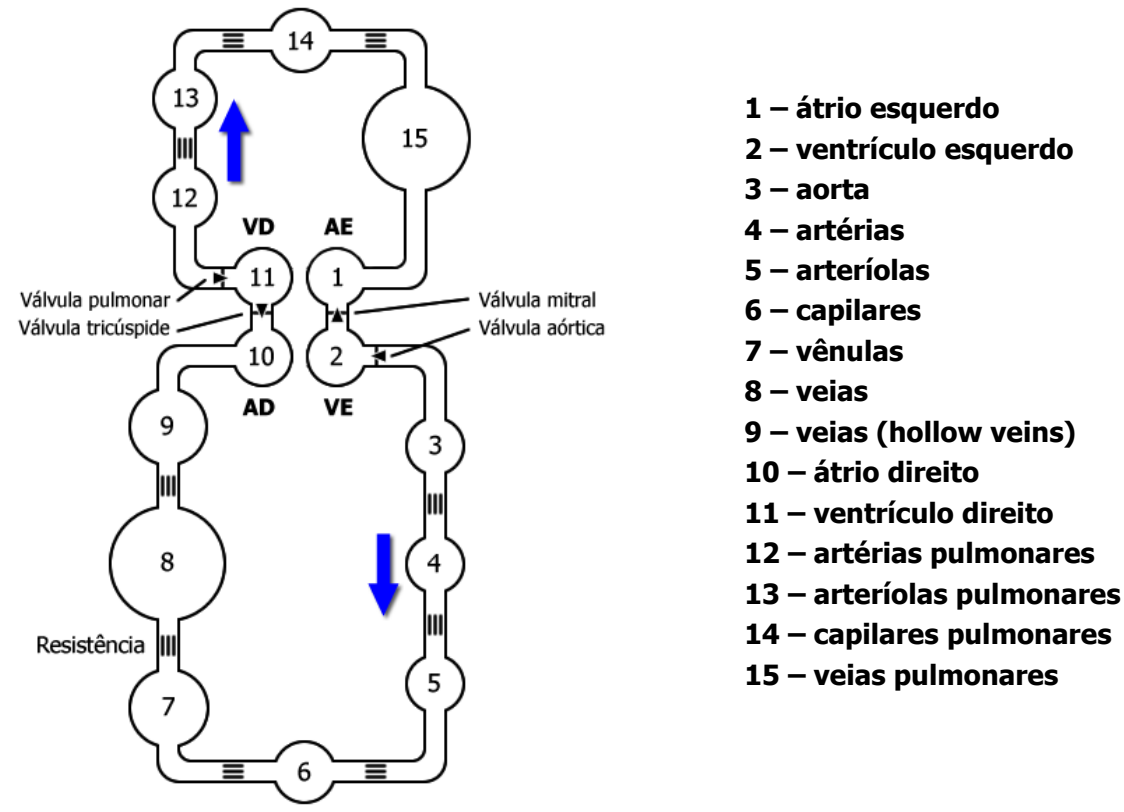

Figura 2-2 Esquema do sistema cardiovascular humano, conforme proposto por Žáček e Krause (1996).

\footnotetext{
${ }^{8}$ JELINEK, J. Hemodynamics of counterpulsation: the study of a lumped-parameter computer model. Journal of Biomechanics, v. 5, p. 511-519, 1972.

${ }^{9}$ LEANING, M. S. et al. Modeling a complex biological system: the human cardiovascular system - 1. Methodology and model description. Trans. Inst. M. C., v. 5, p. 71-86, 1983.

${ }^{10}$ Id. Modeling a complex biological system: the human cardiovascular system -2 . Model validation, reduction and development. Trans. Inst. M. C., v. 5, p. 71-86, 1983.

${ }^{11}$ AVANZOLINI, G. et al. CADCS simulation of the closed-loop cardiovascular system. Int. J. Biomed. Comput., v. 22, p. 3949, 1988.

${ }^{12}$ STERGIOPULOS, N.; YOUNG, D. F.; ROGGE, T. R. Computer simulation of arterial flow with applications to arterial and aortic stenosis. Journal of biomechanics, v. 25, p. 1477-1488, 1992.
} 
Goodwin et al. propuseram em 2004 um modelo ${ }^{13}$ adaptado da tese de Beneken ${ }^{14}$ (1965 apud GOODWIN et al., 2004), que sugerira a modelagem a parâmetros concentrados da fisiologia cardiovascular não controlada. O modelo original era composto por dez compartimentos e era capaz de gerar formas de onda de pressão sangüínea pulsátil. Na versão de 2004, fez-se com que o modelo reagisse apropriadamente a perdas de sangue e administração de volume, a variações de pressão intratorácica, a influências de drogas e finalmente ao controle da circulação através do barorreflexo (ver item 2.2 mais adiante). Além disso, seus parâmetros foram redimensionados de um paciente adulto para um pediátrico, para potencial uso em um simulador em escala natural da fisiologia cardiovascular infantil. Como é possível observar na Figura 2-3, os efeitos de inércia citados anteriormente foram incluídos nas grandes artérias (intratorácicas); no entanto, os compartimentos correspondentes à circulação sistêmica foram colocados em série, sem contabilizar explicitamente a circulação esplâncnica e extra-esplâncnica, cuja importância foi ressaltada por Rowell et al. (1972), Ursino (1998, 2000a, 2003) e Magosso (2001). A Figura 2-4 a seguir apresenta resultados obtidos por Goodwin a partir da simulação deste modelo.

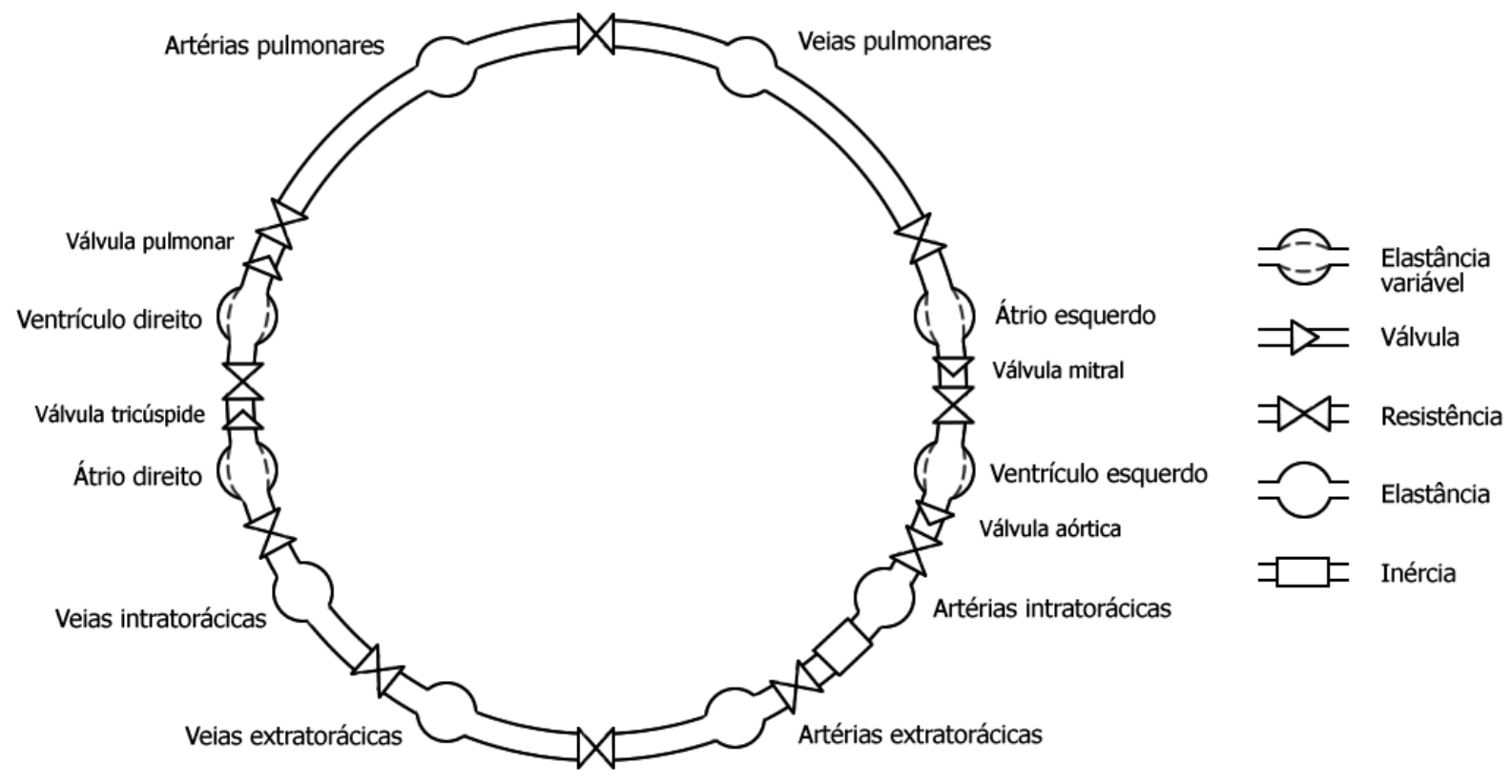

Figura 2-3 Modelo do sistema cardiovascular sugerido por Beneken em 1965 e adaptado por Goodwin em 2004 para uso em simuladores pediátricos em escala natural (adaptado de Goodwin, 2004).

\footnotetext{
${ }^{13}$ Este mesmo modelo também foi utilizado por Van Meurs et al. (2003) para estudo e simulação de defeitos cardíacos em fetos e neonatos.

${ }^{14}$ Beneken, J.E.W. A mathematical approach to cardiovascular function: The uncontrolled human system. Tese (PhD) Medisch Fysisch Institut TNO, Utrecht, The Netherlands, 1965.
} 

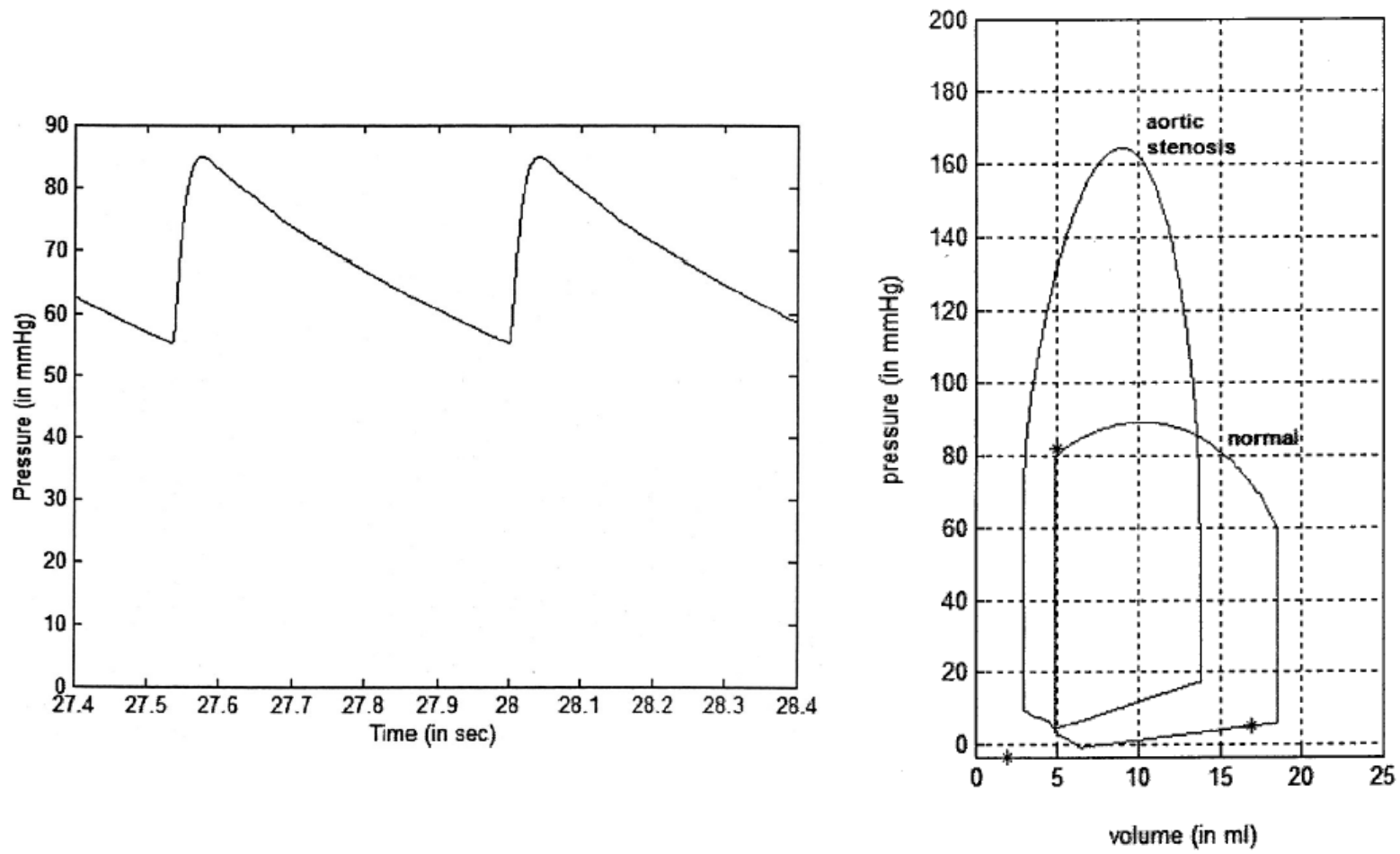

Figura 2-4 Resultados de pressão arterial sistêmica (à esquerda) e alças pressão-volume (à direita) para o ventrículo esquerdo de um paciente com e sem estenose aórtica (GOODWIN, 2004).

Desde 1994, um grupo na universidade de Bologna, na Itália, vem publicando alguns trabaIhos muito promissores na área de modelagem compartimental a parâmetros concentrados do sistema cardiovascular completo. Estes modelos envolvem sempre a auto-regulação, que será tratada no item 2.2 a seguir, sendo apresentada aqui apenas a parte mecânica da fisiologia circulatória. Em 1994, Ursino, Antonucci e Belardinelli propuseram um modelo de seis compartimentos, correspondendo a artérias e veias sistêmicas e pulmonares e os volumes cardíacos direito e esquerdo (representados como fontes de corrente). Este primeiro modelo serviu de base para que, em 1996, Ursino, Fiorenzi e Belardinelli construíssem um novo, capaz de contabilizar os efeitos da pulsatilidade do sangue, além de separar a circulação sistêmica em esplâncnica e extra-esplâncnica (em série). Em 1998, Ursino propôs um novo modelo (ver Figura 2-5), agora com 12 compartimentos, a fim de estudar os efeitos da regulação de curto prazo. Este modelo, que inclui onda de pressão pulsátil (com ventrículos de elastância variável), inertância nas artérias e separação entre vasos esplâncnicos e extra-esplâncnicos (em paralelo, e sujeitos a atuação do barorreflexo), apresenta resultados bastante realistas, e será detalhado mais adiante. Trabalhos posteriores (URSINO; MAGOSSO, 2000a e 2000b; MAGOSSO; URSINO, 2001; MAGOSSO; BIAVATI; URSINO, 2001; URSINO; MAGOSSO, 2003) evoluíram no sentido de 
melhorar a resposta autonômica do sistema, separando órgãos internos (com maior atividade metabólica) e incluindo efeitos da presença dos gases respiratórios $\left(\mathrm{O}_{2}\right.$ e $\left.\mathrm{CO}_{2}\right)$ no sangue.

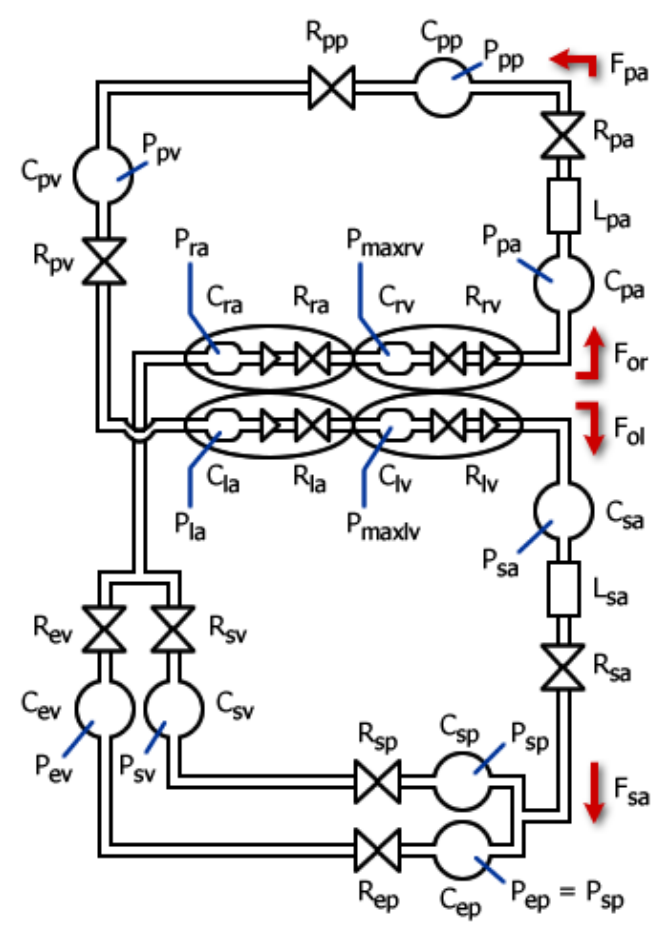

\author{
Símbolos: \\ P - pressões \\ $\mathbf{R}$ - resistências hidráulicas \\ C - complacências \\ $\mathrm{L}-$ inertâncias \\ F - fluxos
}

Índices:

as - artérias sistêmicas

sp - circulação esplâncnica periférica

sv - circulação esplâncnica venosa

ep - circulação extra-esplâncnica periférica

ev - circulação extra-esplâncnica venosa

ra - átrio direito

rv - ventrículo direito

pa - artérias pulmonares

pp - circulação pulmonar periférica

pv - veias pulmonares

la - átrio esquerdo

Iv - ventrículo esquerdo

ol e or - saídas dos ventrículos direito e esquerdo

Pmaxrv e Pmaxlv - pressões ventriculares em condições isométricas

Figura 2-5 Analogia hidráulica do sistema cardiovascular proposta por Ursino em 1998.
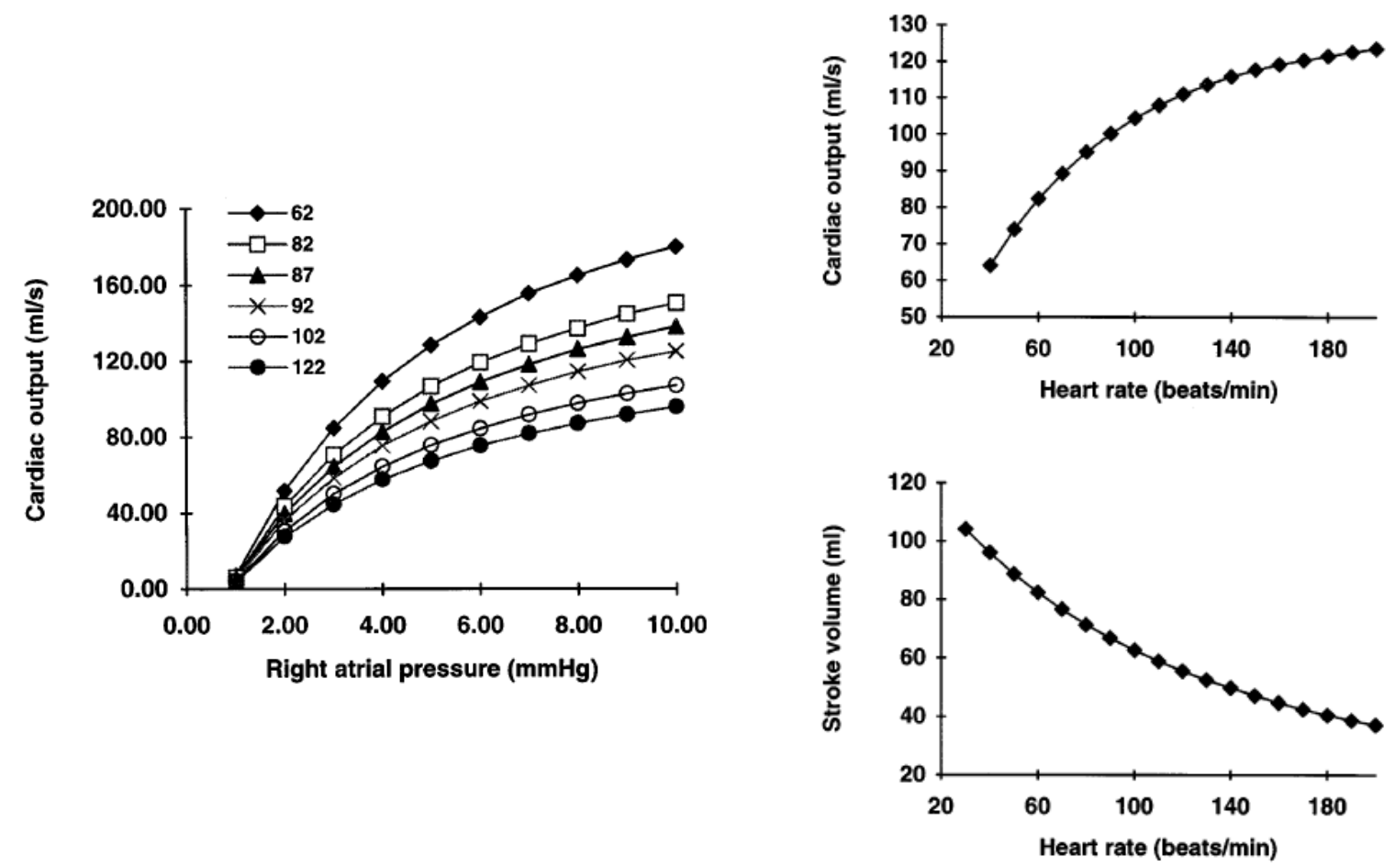

Figura 2-6 Efeitos da atuação do barorreflexo (à esquerda) e da variação da freqüência cardíaca (à direita) obtidos por Ursino (1998). 
A Figura 2-6 acima mostra alguns resultados apresentados no trabalho de Ursino (1998). O gráfico da esquerda mostra os efeitos do barorreflexo carotídeo sobre o débito cardíaco. O valor de débito cardíaco (volume de deslocamento multiplicado pela freqüência cardíaca) foi calculado para diversos níveis de pressão no seio carotídeo, para uma pós-carga constante (pressão sistêmica arterial de $92 \mathrm{mmHg}$ ). Observa-se que a curva de débito cardíaco em função da pressão atrial direita é deslocada para cima conforme a pressão carotídea é reduzida. Os gráficos da direita mostram os efeitos da variação da freqüência cardíaca sobre o débito cardíaco e sobre o volume de deslocamento. Nestas simulações, o sistema de controle atuante sobre a freqüência cardíaca foi desativado, sendo este parâmetro utilizado como entrada externa.

\subsubsection{Modelos Multi-Escalares}

$\mathrm{Na}$ tentativa de contemplar matematicamente toda a complexidade do sistema cardiovascular e de suas interações com outros sistemas fisiológicos, alguns trabalhos têm apontado para uma solução baseada em uma abordagem multi-escalar do sistema. Desta forma, através de soluções numéricas, acoplam-se modelos de diferentes dimensões físicas, sendo eles de parâmetros concentrados ou baseados nas equações de Navier-Stokes para escoamentos uni-, bi- ou tridimensionais, passando por modelos em elementos finitos. Como o sistema circulatório possui natureza multi-escalar, consegue-se assim representar mais precisamente aspectos locais e sistêmicos. Em 1997, Pennati, Bellotti e Fumero utilizaram dados adquiridos através de ultra-som Doppler para estimar parâmetros para um modelo compartimental, seguidos por Formaggia et al. (1999) e Laganà et al. (2004), que propuseram os modelos multi-escalares mostrados na Figura 2-7. Kaye, Primiano e Metaxas utilizaram um modelo multi-escalar (composto por parâmetros concentrados e elementos finitos) para representar as interações cardiopulmonares em um simulador tridimensional para visualização em realidade virtual. 


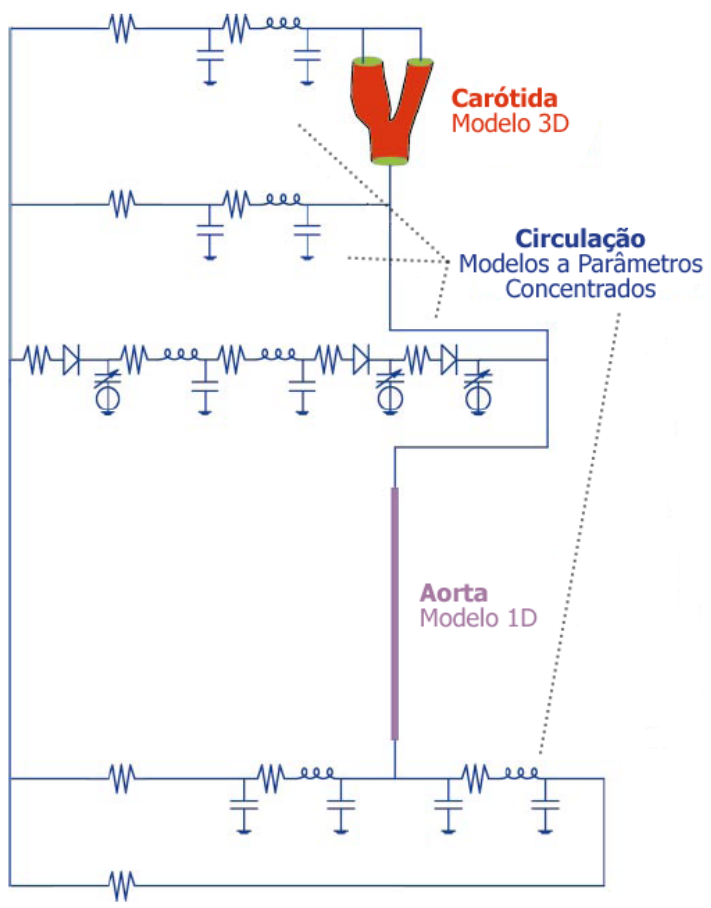

(a)

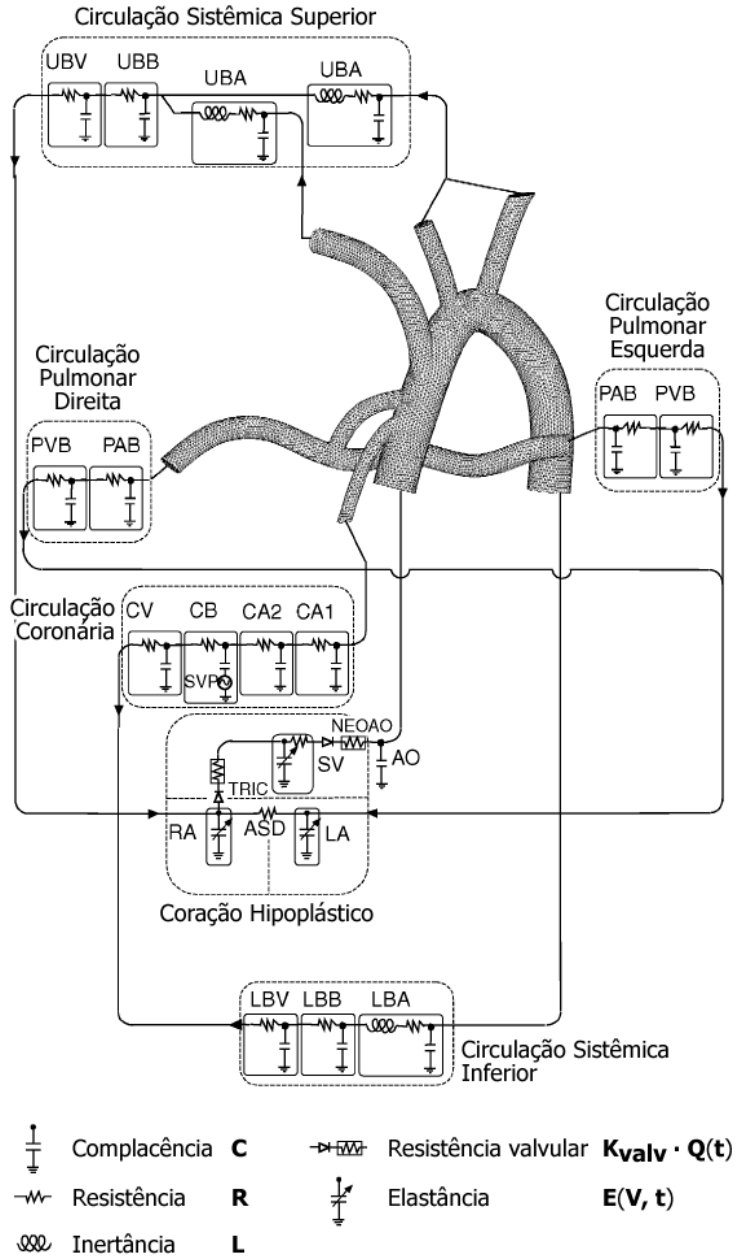

(b)

Figura 2-7 Exemplos de modelos multi-escalares. (a) Modelo proposto por Formaggia et al. (1999), que utiliza as equações de Navier-Stokes para resolver o escoamento do sangue de forma mais precisa. (b) Modelo proposto por Laganà et al. (2004), que utiliza um modelo em elementos finitos da artéria pulmonar.

\subsection{Modelagem dos Mecanismos de Controle e Regulação}

Existem diversos mecanismos de regulação retro-alimentados controlando diferentes aspectos do sistema cardiovascular humano. Dentre eles, diversos são também os mecanismos relacionados com a auto-regulação da pressão sangüínea. No presente trabalho, é de interesse apenas o controle rápido da pressão arterial, mais especificamente o chamado reflexo baroceptor - não serão considerados os demais controles, rápidos ou lentos, envolvidos com as funções de auto-regulação renal, humoral e tecidual. O controle rápido da pressão arterial é realizado através de complexas interações entre informações de retro- e pré-alimentação dos barorreceptores arteriais e cardiopulmonares, que 
se combinam com outros fatores locais e neurais para modular o débito cardíaco (freqüência cardíaca e stroke volume) e a resistência periférica total (HUGHSON et al., 2004).

Os fundamentos fisiológicos relacionados ao barorreflexo já são estudados e conhecidos há algum tempo. Data de 1866 o primeiro relato de uma pesquisa bem sucedida sobre o controle nervoso da pressão sangüínea. O estudo, apresentado por de Cyon e Ludwig $^{15}$ (apud KAREMAKER, 1987) à Royal Saxon Society of Sciences, descrevia um mecanismo neurogênico que atuaria como um controle de retro-alimentação negativo desde o coração até o sistema circulatório e o próprio coração. Eles mostraram que a estimulação do então chamado nervo depressor (no pescoço de um coelho), supostamente originado no coração, levaria a uma queda na freqüência e no débito cardíacos. O único erro cometido por de Cyon e Ludwig foi percebido apenas em 1903, quando Köster e Tschermak ${ }^{16}$ (apud KAREMAKER, 1987) mostraram em um estudo de anatomia que a origem real do nervo estava na parede do arco aórtico. De qualquer forma, o estudo original já apontava dois elementos fundamentais:

- a atividade nervosa aferente, responsável por levar ao sistema nervoso central informações sobre um elemento vital da circulação;

- a atividade nervosa eferente, ditada pelas informações fornecidas pela atividade aferente, e responsável por atuar sobre a freqüência cardíaca, a pressão sangüínea e a distribuição do sangue no sistema vascular.

Em 1927, Hering ${ }^{17}$ (apud KAREMAKER, 1987) mostrou que um segundo receptor de pressão transmitiria um reflexo, até mais poderoso que aquele do arco aórtico, também responsável por desacelerar o coração e diminuir a pressão sangüínea; este receptor se localizaria na parede do seio carotídeo (ver Figura 3-6). Desde então, os componentes do reflexo baroceptor (o caminho aferente, os caminhos eferentes, simpático e parassimpático, e os efetuadores) foram extensivamente analisados e

\footnotetext{
${ }^{15}$ DE CYON, E.; LUDWIG, C. Die Reflexe eines der sensiblen Nerven des Herzens auf diw motorischen der Blutgefässe. Ber. Vehr. Kön. Sächs. Ges. Wiss. Lpz. (Math.-phys. Cl.), v.18, p. 307-328, 1866.

${ }^{16}$ KÖSTER, G.; TSCHERMAK, A. Über den Nervus depressor als Reflexnerv der Aorta. Pflügers Arch. ges. Physiol., v. 93, p. 24-38, 1903.

${ }^{17}$ HERING, H. E. Die Karotissinusreflexe auf Herz und Gefässe. Dresden: Theodor Steinkopf Verlag, 1927.
} 
estudados (KATONA et al., 1970; SCHMIDT; KUMADA; SAGAWA, 1972; ROWELL et al., 1972; GREENE; SHOUKAS, 1986; POTTS; HATANAKA; SHOUKAS, 1996; LANFRANCHI; SOMERS, 2002; LIU et al., 2002), mas sempre prejudicados pelas dificuldades de medição direta dos sinais (RIENZO et al., 1997, LI et al., 2002). Por este motivo, muitos esforços ultimamente têm sido voltados para a modelagem matemática destes mecanismos de regulação, na tentativa de se explicar, através de simulações numéricas, o funcionamento completo do sistema cardiovascular.

Diversos foram os modelos, de variados níveis de complexidade, propostos para elucidar o funcionamento do sistema barorreflexo. Grodins ${ }^{18}$ (apud URSINO, 1994) utilizou em 1959 uma abordagem sistêmica no sistema cardiovascular, que incluía múltiplas equações e a lei de Frank-Starling do coração. Beneken e De Wit ${ }^{19}$ (apud URSINO, 1994) desenvolveram em 1967 um modelo que distinguia entre 19 segmentos periféricos e impunha maior ênfase na descrição mecânica do coração. 0 modelo de Guyton, Coleman e Granger ${ }^{20}$ (1972 apud URSINO, 1994) constitui uma das mais completas descrições do sistema cardiovascular, mas é especialmente focado no controle lento do mesmo. Outras tentativas foram feitas para se modelar os efeitos cumulativos do mecanismo de barorreflexo através de conhecidos métodos da teoria de controle (OTTESEN, 1997). Alguns utilizaram as teorias de controle ótimo, com base na minimização do consumo de energia (NOLDUS ${ }^{21}, 1976$ apud OTTESEN, 1997) ou na minimização do desvio em relação a um valor de referência (ONO et al. ${ }^{22}, 1982$ e KAPPEL; PEER ${ }^{23}$, 1993 apud OTTESEN, 1997) - estes trabalhos não obtiveram sucesso por não se basearem em conhecimentos fisiológicos bem estabelecidos. Em 2004, Fink, Batzel e Kappel voltaram a aplicar as teorias de controle ótimo (com função objetivo de minimização de desvio de múltiplos

${ }^{18}$ GRODINS, F. S. Integrative cardiovascular physiology: A mathematical synthesis of cardiac and blood vessel hemodynamics. Quarterly Review of Biology, v. 34, p. 93-116, 1959.

${ }^{19}$ BENEKEN, J. E. W.; DE WIT, B. A physical approach to hemodynamic aspects of the human cardiovascular system. In: REEVE, E. B.; GUYTON, A. C. (Eds.) Physical bases of circulatory transport: Regulation and exchange. Philadelphia: Saunders, 1967. p. 1-45.

${ }^{20}$ GUYTON, A. C.; COLEMAN, T. G.; GRANGER, H. J. Circulation: Overall regulation. Annual Review of Physiology, v. 34, p. 13-46, 1972.

${ }^{21}$ NOLDUS, E. Optimal control aspects of left ventricular ejection dynamics. Journal of Theoretical Biology, v. 63, p. 275309, 1976.

${ }^{22}$ ONO, K.; UOZUMI, T.; YOSHIMOTO, C.; KENNER, T. The optimal cardiovascular regulation of the arterial blood pressure. In: KENNER, T.; BUSSE, R.; HINGHOFER-SZALKAY (Eds.). Cardiovascular system dynamics: Model and measurements. Plenum Press, 1982. p. 119-139.

${ }^{23}$ KAPPEL, F.; PEER, R. A mathematical model for fundamental regulation processes in the cardiovascular system. Journal of Mathematical Biology, v. 31, p. 611-631, 1993. 
valores de referência) para estudar o estresse ortostático - agora com maior sucesso. Outros trabaIhos (RIDEOUT ${ }^{24}$, 1991 e THAM ${ }^{25}, 1988$ apud OTTESEN, 1997) utilizaram princípios da teoria de controle linear, apesar de o reflexo baroceptor ser fortemente não linear (GUYTON; HALL, 1997; URSINO; ANTONUCCI; BELARDINELLI, 1994; OTTESEN, 1997 e 2000). Recentemente, o controle da função cardiovascular também foi modelado através de redes neurais (de CAÑETE et al., 2002), apresentando resultados interessantes.

Em 1994, Ursino, Antonucci e Belardinelli propuseram um modelo matemático do barorreflexo seio carotídeo (ficaram ausentes os barorreceptores cardiopulmonares e do arco aórtico), incorporado a um modelo simplificado (não-pulsátil) do sistema cardiovascular, com a finalidade de esclarecer o papel das variações ativas da capacidade venosa no controle rápido da pressão arterial. O modelo considerava as não linearidades do mecanismo, e incluía nos atuadores a resistência arterial sistêmica, a freqüência e a contratilidade cardíacas, além da já citada capacidade venosa (manifestada através do volume venoso não distendido e da complacência venosa sistêmica). Um novo modelo (URSINO; FIORENZI; BELARDINELLI, 1996) passou a incluir a simulação da pressão pulsátil, através de ventrículos com elastância variável. Schmidt, Kumada e Sagawa já haviam observado em 1972 que a estimulação dos barorreceptores carotídeos por meio do aumento da atividade pulsátil resultaria em um aumento da descarga do receptor, desenvolvimento de hipotensão arterial e uma redução no ganho estático do receptor.

Em 1997, Ottesen propôs uma modelagem direta do mecanismo de realimentação, baseada em teorias fisiológicas bem estabelecidas e fatos empíricos, introduzindo um atraso, além das nãolinearidades já citadas (a modelagem foi revista e melhorada em 2000, pelo próprio autor). Os efeitos cronotrópicos (relacionados às alterações da freqüência cardíaca) do mecanismo foram modelados explicitamente, sendo divididos em uma parte que descreve a atividade (sinais elétricos) das fibras nervosas, e outra parte que descreve a variação da freqüência cardíaca como uma função dos sinais simpáticos e parassimpáticos - é entre a primeira e a segunda parte que se situa o atraso citado ante-

\footnotetext{
${ }^{24}$ RIDEOUT, V. Mathematical and computer modeling of physiological systems. Medical Physics Publishing, 1991.

${ }^{25}$ THAM, R.-Y. A study of effects of halothane on canine cardiovascular system and baroreceptor control, 1988. Xerographic printed by UMI Dissertation Services, A Bell \& Howell Company, 1995.
} 
riormente. O efeito inotrópico, relacionado à contratilidade do músculo cardíaco, também foi considerado, mas com atenção reduzida.

O modelo de Ursino, Fiorenzi e Belardinelli, de 1996, serviu de base para alguns outros trabaIhos sobre o sistema baroceptor. Em 1998, Ursino publicou um trabalho que incluía naquele modelo de 1996 uma melhor descrição do sistema cardiovascular (com o aumento do número de compartimentos), uma descrição consistente dos efeitos inotrópicos, e a separação da circulação sistêmica em esplâncnica e extra-esplâncnica, mostrando que estas exibem resposta diferenciada ao barorreflexo. Sobre este último modelo, Ursino e Magosso incluíram, em 2000, outros mecanismos de reflexo, como os quimioceptores periféricos (tanto nas vias aferentes quanto eferentes), os receptores de deformação pulmonar, o efeito direto da hipóxia no sistema nervoso central e ainda a separação das redes vasculares para os órgãos com maiores necessidades metabólicas. O modelo de 1998 ainda foi utilizado em 2001 (por Magosso, Biavati e Ursino) para estudar o papel do barorreflexo na instabilidade do sistema cardiovascular, e em 2003 (por Ursino e Magosso) para analisar a variabilidade da freqüência cardíaca.

\subsection{Simulação}

$\mathrm{Na}$ literatura referente à simulação didática do sistema cardiovascular predominam os textos sobre os chamados simuladores de escala natural. Estes simuladores consistem em bonecos em tamanho natural que simulam, além do sistema circulatório completo (incluindo mecanismos de reflexo baro- e quimioceptor, e muitas vezes, interações gasosas e reação à infusão de medicamentos), outros sistemas fisiológicos, como o respiratório. No entanto, o interesse deste trabalho está na simulação baseada em telas (screen-based simulation), pois o intuito do simulador em desenvolvimento é promover a instrução básica dos estudantes de saúde, através de uma ferramenta acessível e de fácil distribuição. De qualquer forma, como alguns dos conceitos desenvolvidos para as simulações em escala natural aplicam-se também às simulações de tela, em certos momentos desta seção serão apresentados trabalhos do primeiro tipo.

Em 2004, Gaba introduziu uma classificação para as atuais e futuras aplicações de simulação, estabelecendo 11 dimensões que representam seus diferentes atributos. Cada dimensão corresponde 
a um aspecto da simulação, subdividido em categorias que a caracterizem. $O$ intuito destas dimensões é fornecer um panorama que facilite a compreensão da diversidade das aplicações de simulação em medicina e saúde. As definições convenientes serão tratadas no decorrer deste texto, mas, para evitar o comprometimento de sua clareza, as categorias propostas por Gaba não serão explicitamente descritas (caso o leitor deseje, uma breve descrição foi incluída no Apêndice A). Gallagher et al. (2005) enfatiza as diferenças entre simulação aplicada a treinamento e a educação, que costumam ser utilizadas erroneamente com o mesmo sentido. A educação (alvo desta dissertação) possui um caráter mais associado à didática, e à comunicação e transferência de conhecimento, enquanto o treinamento envolve a aquisição de habilidades e competências cognitivas ou psicomotoras. O autor propõe ainda um template (também apresentado no Apêndice A) para o desenvolvimento de um currículo que inclua adequadamente o uso de simuladores, tanto em educação quanto em treinamento.

\subsubsection{Simuladores de Tela (screen-based) e Simuladores de Escala Natural (full-scale)}

Os simuladores de paciente surgiram para suprir as necessidades específicas de treinamento em anestesia, na forma dos simuladores em escala natural, ainda na década de sessenta (van MEURS; GOOD; LAMPOTANG, 1997). Já nesta época, estudos como o de Abrahamson, Denson e Wolf (1969) demonstravam sua eficiência no aprendizado dos residentes. Mais recentemente, estes simuladores ganharam níveis de complexidade mais elevados, com a incorporação de modelos mais precisos, passando a abranger um maior número de sistemas fisiológicos. Isso possibilitou sua utilização no treinamento de procedimentos clínicos em geral, com o objetivo de reduzir os riscos aos pacientes reais (GABA, 2004; van MEURS; GOOD; LAMPOTANG, 1997; van MEURS et al., 2003; BEYEA; KOBOKOVICH, 2004).

Com a evolução progressiva das interfaces gráficas computacionais, tornou-se praticável o desenvolvimento de simuladores de paciente baseados em telas, existentes apenas como aplicativos no computador. Estes simuladores podem ser divididos em dois tipos fundamentais: os simuladores imersivos, baseados em ambientes tridimensionais e realidade virtual (KAYE; PRIMIANO; METAXAS, 1998; NAKAO et al., 2002; CAVAZZA; SIMO, 2003; GALLAGHER et al., 2005), e os simuladores de fisiologia, que utilizam interfaces interativas, com tutoriais, textos e animações, com finalidades didáticas mais 
elementares (GERSTING; ROTHE, 2002; ROTHE; GERSTING, 2002; MUKKAMALA; TOSKA; COHEN, 2003) - é neste grupo que o simulador aqui proposto se enquadra.

\subsubsection{Simulação Controlada por Roteiros e Simulação Baseada em Modelos}

Inicialmente, devem-se diferenciar duas diferentes abordagens que podem ser utilizadas para gerar automaticamente as respostas fisiológicas de um paciente simulado - a simulação controlada por roteiros e a simulação baseada em modelos matemáticos (van MEURS; GOOD; LAMPOTANG, 1997). A seleção depende do tipo de aplicação desejada, uma vez que ambas podem possuir vantagens e desvantagens em relação à outra.

A primeira abordagem baseia-se em roteiros (scripts) de simulação, que consistem em conjuntos de comandos que fazem com que o simulador se comporte da maneira especificada. Estes roteiros devem prever as ações do operador e responder com o comportamento fisiológico esperado do paciente (van MEURS; GOOD; LAMPOTANG, 1997). Este tipo de abordagem é mais utilizado em simuladores de escala natural, onde os roteiros podem descrever respostas a eventos que podem ser caracterizados por sua ocorrência e seqüência temporal, como em procedimentos de entubação endotraqueal e laringoscopia (GABA, 2004; van MEURS et al., 2003). A vantagem deste tipo de abordagem reside na geração de respostas explícitas e fiéis, armazenadas na forma de trechos discretos correspondentes aos possíveis eventos resultantes das ações do operador. Desta forma, a resposta obtida é sempre real, coletada através de experimentos ou simulada através de um roteiro específico para aquele evento. No entanto, em casos de variação com incrementos muito pequenos (podendo-se extrapolar para casos de variação contínua), uma enorme quantidade de eventos e respostas prontas teria que estar prevista. Desta forma, torna-se praticamente impossível que uma simulação roteirizada consiga antecipar todas as possíveis situações e variações de parâmetros, especialmente em sistemas complexos como o respiratório e o cardiovascular, onde uma pequena alteração de um parâmetro pode acarretar em uma significativa mudança em outro.

Contrariamente ao caso anterior, as simulações baseadas em modelos matemáticos são capazes de representar variáveis como valores contínuos, que evoluem com o tempo. Mais do que isso, estas simulações podem reagir prontamente a uma diversidade de entradas de controle, representan- 
do as possíveis interações entre diferentes subsistemas fisiológicos (van MEURS; GOOD; LAMPOTANG, 1997). Além disso, pode-se ir até as causas envolvidas nos fenômenos estudados, permitindo que uma simples alteração desencadeie uma seqüência de eventos que altere todo o estado inicial do sistema. Por exemplo, a perda de sangue no sistema cardiovascular (redução do volume sangüíneo) provoca uma queda na pressão arterial, que deixa de atuar nos baroreceptores; isso resulta no aumento da ação simpática e na diminuição da ação parassimpática, que por sua vez, induz o retorno dos valores normais de pressão. Toda esta seqüência de eventos e interações entre subsistemas seria virtualmente impossível de ser simulada através de roteiros.

Mais recentemente, uma terceira abordagem vem sendo utilizada, principalmente em simuladores de escala natural. Dados a grande variedade de estados (normais ou patológicos) e subsistemas geralmente envolvidos neste tipo de aplicação, uma abordagem puramente matemática seria extremamente complicada, podendo deixar de atender certos aspectos. Por outro lado, uma abordagem exclusivamente baseada em roteiros apresentaria respostas limitadas, até mesmo insuficientes (van MEURS; GOOD; LAMPOTANG, 1997). Desta forma, a aliança dos aspectos mais convenientes do controle por roteiros com o poder das simulações matemáticas poderia levar ao desenvolvimento de simuladores que envolvessem uma maior quantidade de subsistemas fisiológicos.

\subsubsection{Simulação Didática do Sistema Cardiovascular}

Durante o levantamento da literatura sobre o sistema cardiovascular, notou-se existirem poucas publicações sobre simulação didática, para finalidades educacionais. $O$ trabalho de van Meurs et al. (2003), e sua continuação publicada por Goodwin et al. (2004), baseados na tese de Beneken ${ }^{26}$ (1965 apud GOODWIN et al., 2004), apenas sugerem seu uso em simuladores baseados em telas, mas não descrevem implementações eventualmente já realizadas. Os trabalhos de Mukkamala e Cohen (2001) e Mukkamala, Toska e Cohen (2003) resultaram no desenvolvimento de um simulador do sistema cardiovascular (RCVSIM ${ }^{27}$ ) baseado em MatLab para o sistema operacional Linux. No entanto,

\footnotetext{
${ }^{26}$ BENEKEN, J.E.W. A mathematical approach to cardiovascular function: The uncontrolled human system. Tese (PhD) Medisch Fysisch Institut TNO, Utrecht, The Netherlands, 1965.

${ }^{27}$ RCVSIM - Research Cardiovascular Simulator. Disponível em: <http://www.physionet.org/physiotools/rcvsim/>.
} 
a finalidade deste simulador é a pesquisa, principalmente em engenharia biomédica, e seu uso requer certos conhecimentos de ferramentas computacionais específicas normalmente não possuídos por estudantes de fisiologia.

Baseados no pioneiro MacMan, apresentado em 1973 por Dickinson, Goldsmith e Sackett ${ }^{28}$ (apud MICHAEL, 2001), Rovick e Brenner ${ }^{29}$ (apud MICHAEL, 2001) desenvolveram o Heartsim, que consistia basicamente na incorporação de alguns tutoriais e exercícios ao primeiro (MICHAEL, 2001). Este último, por sua vez, serviu de base para o desenvolvimento do CIRCSIM $^{30}$ (ROVICK; MICHAEL, 1986 apud MICHAEL, 2001), que marcou a transição para os PCs padrão x86. Todos estes simuladores eram baseados em modelos simplificados, com interfaces simplistas, apenas numéricas (MICHAEL, 2001). Desde o lançamento do CIRCSIM, iniciou-se o desenvolvimento do projeto CIRCSIM-Tutor, com a finalidade de construir um sistema de tutoria para utilização por estudantes de medicina do primeiro ano no aprendizado do controle da pressão sangüínea. O modelo utilizado no projeto continua simples, pois o foco deste projeto está mesmo no ensino e na didática, sendo o simulador baseado em linguagem natural, de modo que o aluno deve conversar com o programa ${ }^{31}$.

Também motivados pelos conceitos de aprendizado consistente propostos por Michael (2001), Rothe e Gersting apresentaram em 2002 um tutorial interativo baseado em um modelo matemático simples (de 5 compartimentos, não pulsátil) do sistema cardiovascular - o chamado projeto CVI (Cardiovascular Interactions). O simulador possui uma interface em janelas, com os parâmetros numéricos permanentemente expostos ao usuário (ver Figura 2-8). O programa é capaz de plotar gráficos dos parâmetros, mas apenas para simulações com período limitado, e não em tempo real.

\footnotetext{
${ }^{28}$ DICKINSON C.J.; GOLDSMITH C.H.; SACKETT D. L. MACMAN: A digital computer model for teaching some basic principles of Hemodynamics. Journal of Clinical Computing, v. 2, p. 42-50, 1973.

${ }^{29}$ ROVICK, A. A.; BRENNER, L. Heartsim: a cardiovascular simulation with didactic feedback. Physiologist, v. 25: 343, 1982.

${ }^{30}$ ROVICK, A. A.; MICHAEL, J. A. CIRCSIM: An IBM PC computer teaching exercise on blood pressure regulation. XXX International Union of Physiological Sciences Congress. Vancouver, Canada, 1986.

${ }^{31}$ CIRCSIM-TUTOR PROJECT. Disponível em <http://www.cs.iit.edu/ circsim/>.
} 


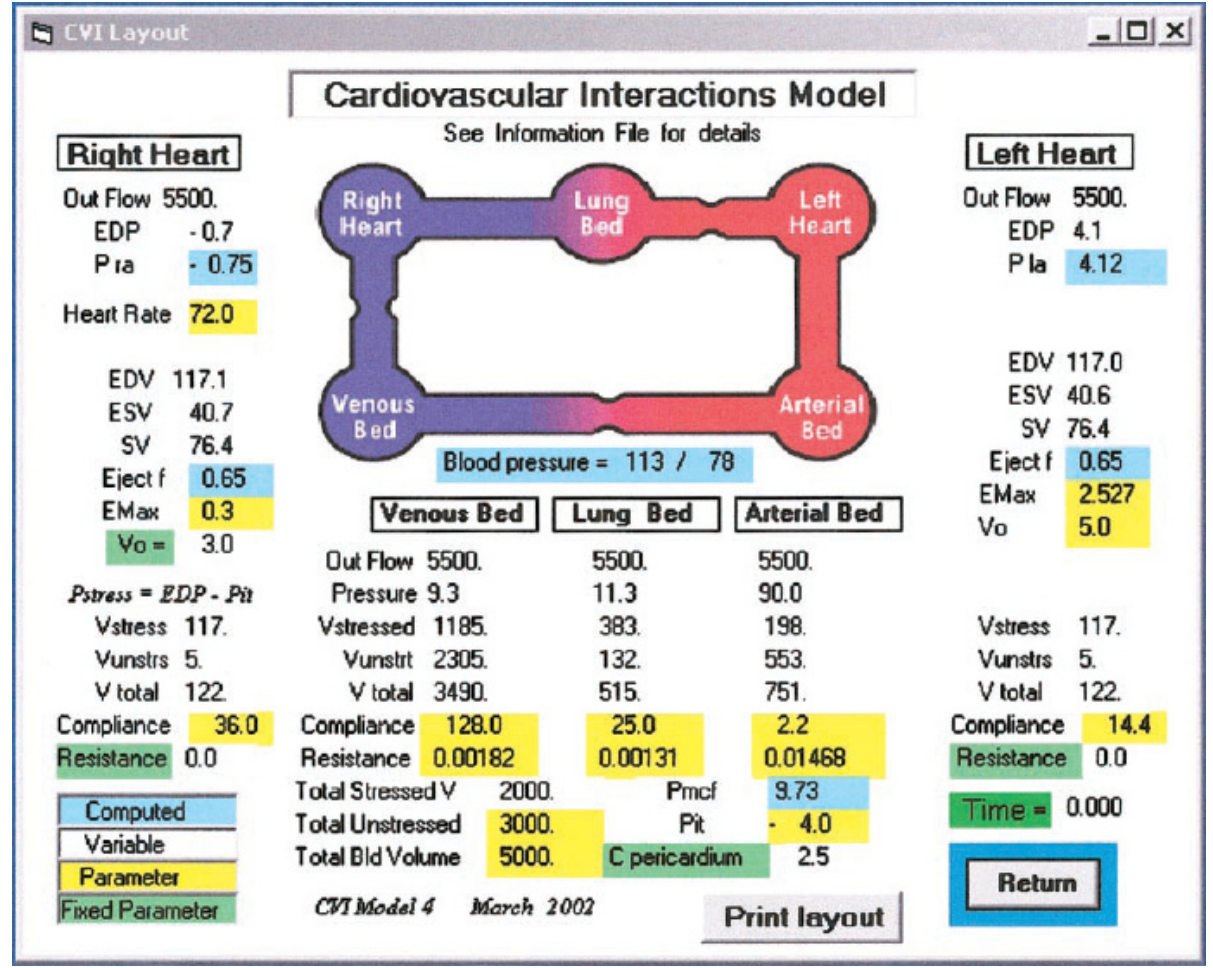

Figura 2-8 Interface principal do simulador CVI (ROTHE; GERSTING, 2002) 


\section{Modelagem e Simulação do Sistema Cardiovascular Controlado}

A proposta desta dissertação consiste no desenvolvimento de um simulador didático do sistema cardiovascular humano, controlado através do mecanismo de reflexo baroceptor e capaz de atualizar seus parâmetros em tempo real. Para que isto seja alcançado, um modelo matemático da fisiologia deve ser executado no núcleo do programa, de modo a se conseguir as respostas desejadas. Desta forma, como o objetivo principal do trabalho é a construção do simulador, e sabendo da existência na literatura de trabalhos de modelagem mais consolidados, optou-se inicialmente por utilizar um modelo pronto, ajustado para atender às necessidades do projeto.

O modelo selecionado para servir de base para este trabalho foi o proposto por Ursino em 1998. Como características decisivas para esta escolha, podem ser citadas:

- a separação da circulação em arterial e venosa, e ainda entre grandes e pequenos vasos, com efeitos de inércia considerados nas grandes artérias;

- a descrição dos ventrículos como bombas pulsáteis, através de modelos de elastância variável;

- a separação da circulação sistêmica em esplâncnica e extra-esplâncnica, importante para o sistema de controle;

- a inclusão das atividades simpáticas e parassimpáticas no sistema de barorreflexo, atuando sobre a freqüência e a contratilidade cardíacas e sobre resistências e volumes não distendidos.

Além destes fatores, o trabalho original apresentou resultados interessantes, bastante próximos aos valores fisiológicos esperados - isto poderá ser observado através de alguns gráficos comparativos no Capítulo 4. A única ressalva, desde o princípio, era a consideração por parte de Ursino de átrios passivos, não-pulsáteis, compostos por resistência e complacência constantes. Receava-se que, por este motivo, as curvas dos átrios não ficassem adequadas aos valores fisiológicos esperados, comprometendo os objetivos didáticos do simulador final. Conforme será descrito mais adiante, as simula- 
ções da reprodução do modelo base confirmaram esta suspeita, gerando a necessidade de adição de algumas novas equações e parâmetros ao mesmo.

Este capítulo descreve toda a modelagem do sistema controlado, incluindo a parte reproduzida a partir do trabalho de Ursino e as contribuições referentes aos átrios ativos. São apresentadas as leis e equações básicas, e o modelo é avaliado quanto a sua adequação às finalidades propostas. 0 texto foi organizado em três principais partes, que correspondem à modelagem da planta com átrios passivos, à modelagem do sistema de controle e à modelagem dos átrios ativos.

A primeira parte trata do sistema vascular (o modelo hidráulico), sendo subdividida entre os compartimentos vasculares (passivos) e o coração (átrios passivos e ventrículos ativos, gerando pressão pulsátil). A segunda parte descreve o modelo do mecanismo de reflexo baroceptor, com subdivisões relacionadas às vias aferentes (que levam os sinais dos receptores ao sistema nervoso central), às vias eferentes (que retornam os sinais aos atuadores) e aos atuadores (que exercem as ações regulatórias sobre o sistema). A terceira parte descreve o modelo dos átrios ativos, equacionado com base nas relações sugeridas para os ventrículos (na primeira parte) e no modelo sugerido por Goodwin et al. (2004), mencionado na revisão bibliográfica. Esta parte inclui ainda o ajuste de parâmetros realizado, que também teve como base o mencionado trabalho, acrescido de extensivas simulações numéricas para adaptação a valores da literatura. Os resultados obtidos a partir das simulações numéricas do modelo completo, realizadas nos softwares Matlab e Simulink, são mostrados no Capítulo seguinte.

\subsection{Sistema Vascular}

O modelo do sistema vascular compreende 12 compartimentos, sendo que oito deles correspondem aos elementos vasculares e quatro correspondem às câmaras do coração. Os compartimentos são compostos por resistências, complacências e (no caso das grandes artérias) inertâncias hidráulicas. A Figura 3-1 apresenta o modelo hidráulico, descrito em detalhes nas seções a seguir. 


\subsubsection{Compartimentos Vasculares}

O modelo do sistema vascular inclui oito compartimentos passivos, conforme mostrado na Figura 3-1 abaixo.

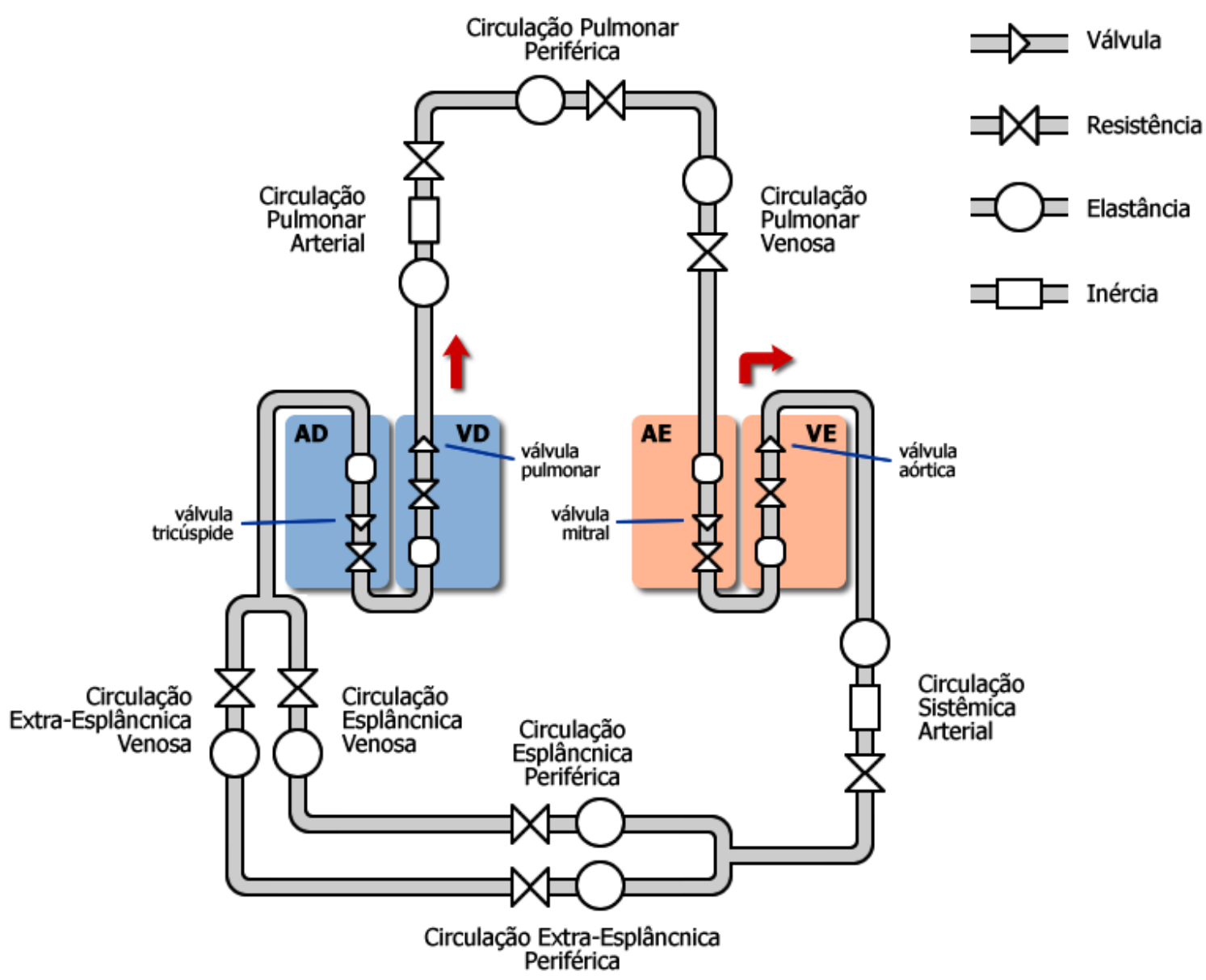

Figura 3-1 Modelo hidráulico do sistema cardiovascular, apresentando os elementos que compõem os compartimentos vasculares. No diagrama, procurou-se preservar certa associação anatômica nas posições dos elementos. As setas indicam o sentido do fluxo sangüíneo.

A circulação sistêmica corresponde a cinco destes compartimentos, diferenciados em artérias sistêmicas (as grandes artérias, incluindo aorta e carótida), circulação periférica esplâncnica (referente aos órgãos internos) e extra-esplâncnica (referente aos demais componentes), e circulação venosa esplâncnica e extra-esplâncnica. A circulação pulmonar é representada pelos outros três compartimentos, correspondendo à artéria pulmonar, à circulação pulmonar periférica e às veias pulmonares. Todos os compartimentos são compostos por pelo menos uma resistência hidráulica (para contabilizar a queda de pressão no compartimento), uma complacência (que descreve a quantidade de sangue armazenado no compartimento a uma dada pressão) e um volume não distendido (correspondente a uma pressão relativa nula no compartimento). Para os compartimentos que representam as grandes 
artérias, onde os efeitos da aceleração do sangue são mais significativos, foi também incluída uma inertância.

Como o modelo será de parâmetros concentrados, será considerado que o fluido é Newtoniano e o escoamento é unidirecional. As relações básicas que descrevem o sistema podem ser obtidas através das leis de conservação de massa (aplicada a todas as complacências) e de equilíbrio de forças (aplicada nas grandes artérias) - ou ainda através de analogia elétrica.

\section{Circulação Pulmonar}

Define-se como $\mathbf{P}$ a pressão intravascular no compartimento, $\mathbf{V}$ o volume contido, $\mathbf{V}_{\mathbf{u}}$ o volume não distendido, $\mathbf{Q}$ a vazão sangüínea, $\mathbf{R}$ a resistência, $\mathbf{C}$ a complacência e $\mathbf{L}$ a indutância. As siglas utilizadas para nomear os compartimentos estão resumidas na Figura 3-2 abaixo.

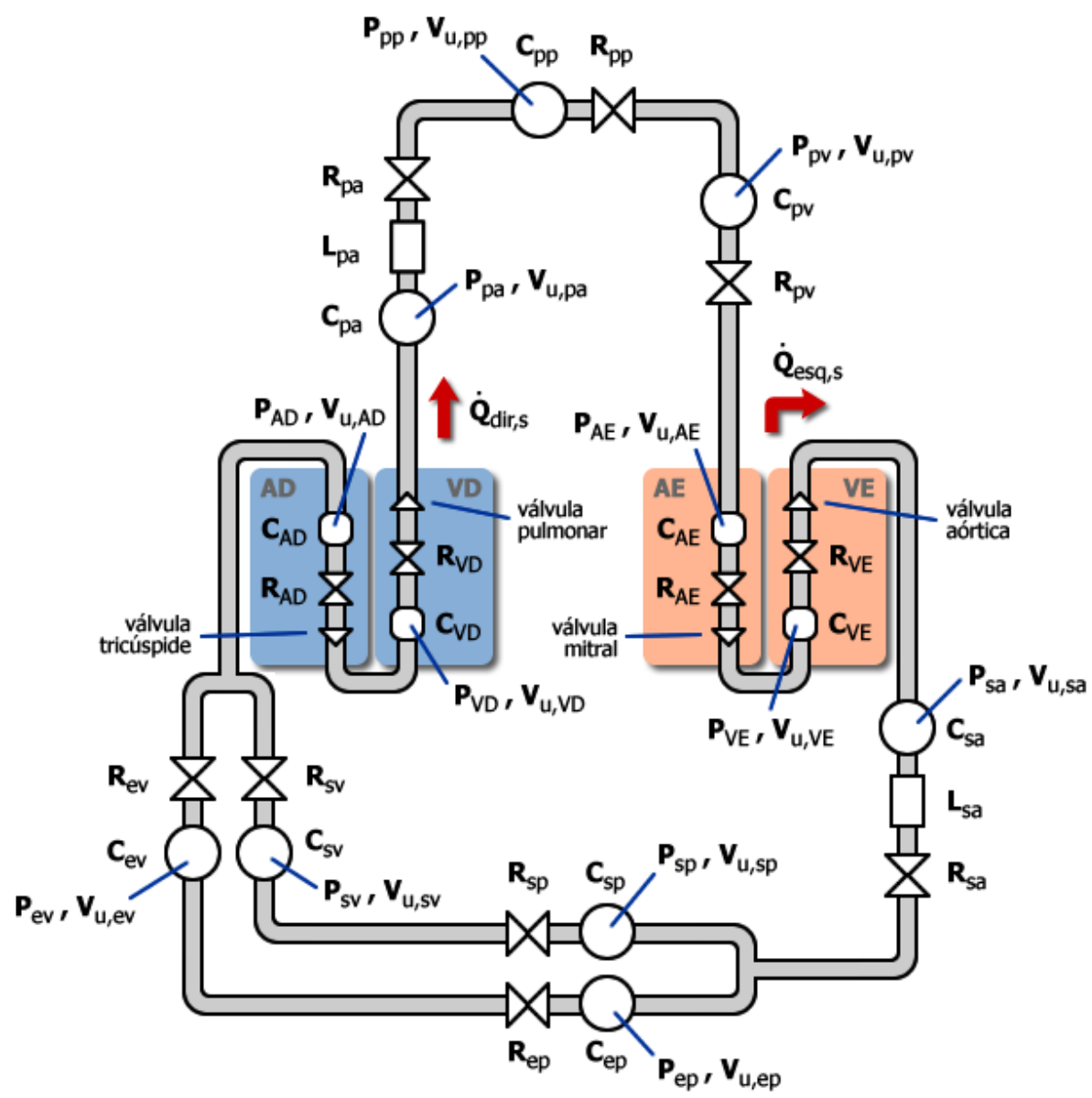

Figura 3-2 Modelo hidráulico mostrando as variáveis que o descrevem. As câmaras do coração são identificadas por índices em letras maiúsculas.

A conservação de massa nas artérias pulmonares (subscrito pa) nos fornece: 


$$
\mathbf{C}_{\mathbf{p a}} \frac{\mathbf{d P}}{\mathbf{d t}}=\dot{\mathbf{Q}}_{\mathrm{dir}, \mathbf{s}}-\dot{\mathbf{Q}}_{\mathbf{p a}}
$$

onde $\dot{\mathbf{Q}}_{\text {dir,s }}$ representa o débito cardíaco (fluxo de saída) do ventrículo direito. O equilíbrio de forças nas artérias pulmonares resulta em:

$$
\mathbf{L}_{p a} \frac{\mathbf{d} \dot{Q}_{p a}}{\mathbf{d t}}=\mathbf{P}_{p a}-\mathbf{P}_{p p}-\mathbf{R}_{p a} \dot{Q}_{p a}
$$

sendo que o subscrito pp indica circulação pulmonar periférica, $\mathbf{L}_{\mathbf{p a}}$ é a indutância das artérias pulmonares e o termo $\mathbf{R}_{\mathbf{p a}} \dot{\mathbf{Q}}_{\mathbf{p a}}$ representa a perda de carga no segmento. A conservação de massa na circulação pulmonar periférica nos dá:

$$
C_{p p} \frac{d P_{p p}}{d t}=\dot{Q}_{p a}-\frac{P_{p p}-P_{p v}}{R_{p p}}
$$

onde o índice pv representa a circulação pulmonar venosa, e o segundo termo do lado direito da Equação 3-3 corresponde à vazão neste compartimento, $\dot{\mathbf{Q}}_{\mathbf{p v}}$ :

$$
\dot{\mathbf{Q}}_{\mathbf{p v}}=\frac{\mathbf{P}_{\mathbf{p p}}-\mathbf{P}_{\mathbf{p v}}}{\mathbf{R}_{\mathbf{p p}}}
$$

Finalmente, temos da conservação de massa nas veias pulmonares:

$$
\mathbf{C}_{\mathbf{p v}} \frac{\mathbf{d P} \mathbf{P}_{\mathbf{p v}}}{\mathbf{d t}}=\frac{\mathbf{P}_{\mathrm{pp}}-\mathbf{P}_{\mathbf{p v}}}{\mathbf{R}_{\mathrm{pp}}}-\frac{\mathbf{P}_{\mathrm{pv}}-\mathbf{P}_{\mathrm{AE}}}{\mathbf{R}_{\mathrm{pv}}}
$$

com o subscrito AE representando o átrio esquerdo.

\section{Circulação Sistêmica}

A conservação de massa nas artérias sistêmicas (subscrito sa) resulta:

$$
\mathbf{C}_{\text {sa }} \frac{\mathbf{d P}}{\mathbf{d t}}=\dot{\mathbf{Q}}_{\text {esq,s }}-\dot{\mathbf{Q}}_{\mathbf{s a}}
$$

onde $\dot{\mathbf{Q}}_{\text {esq,s }}$ representa o débito cardíaco do ventrículo esquerdo. O equilíbrio de forças nas artérias sistêmicas fornece:

$$
\mathbf{L}_{\text {sa }} \frac{\mathbf{d} \dot{\mathbf{Q}}_{\mathrm{sa}}}{\mathbf{d t}}=\mathbf{P}_{\mathrm{sa}}-\mathbf{P}_{\mathrm{sp}}-\mathbf{R}_{\mathrm{sa}} \dot{\mathbf{Q}}_{\mathrm{sa}}
$$


onde o subscrito sp indica circulação esplâncnica periférica e o termo $\mathbf{R}_{\mathbf{s a}} \dot{\mathbf{Q}}_{\mathbf{s a}}$ representa a perda de carga no segmento.

Sendo a pressão periférica extra-esplâncnica $\left(\mathbf{P}_{\mathbf{e p}}\right)$ igual à pressão periférica esplâncnica $\left(\mathbf{P}_{\mathbf{s p}}\right)$, a conservação de massa na circulação periférica resulta em:

$$
\left(C_{\text {sp }}+\mathbf{C}_{\text {ep }}\right) \frac{\mathbf{d P _ { \text { sp } }}}{\mathbf{d t}}=\dot{\mathbf{Q}}_{\mathbf{s a}}-\frac{\mathbf{P}_{\text {sp }}-\mathbf{P}_{\text {sv }}}{\mathbf{R}_{\mathbf{s p}}}-\frac{\mathbf{P}_{\text {ep }}-\mathbf{P}_{\text {ev }}}{\mathbf{R}_{\text {ep }}}
$$

onde sv indica a circulação esplâncnica venosa e ev indica a circulação extra-esplâncnica venosa. Define-se assim a vazão esplâncnica venosa (segundo termo do lado direito da Equação 3-8)

$$
\dot{\mathbf{Q}}_{\mathbf{s v}}=\frac{\mathbf{P}_{\mathbf{s p}}-\mathbf{P}_{\mathbf{s v}}}{\mathbf{R}_{\mathbf{s p}}}
$$

e a vazão extra-esplâncnica venosa (terceiro termo do lado direito da Equação 3-8)

$$
\dot{\mathbf{Q}}_{\mathbf{e v}}=\frac{\mathbf{P}_{\mathbf{e p}}-\mathbf{P}_{\mathbf{e v}}}{\mathbf{R}_{\mathbf{e p}}}
$$

A conservação de massa para a circulação extra-esplâncnica venosa fornece:

$$
\mathbf{C}_{\mathrm{ev}} \frac{\mathbf{d P _ { \text { ev } }}}{\mathbf{d t}}=\frac{\mathbf{P}_{\mathbf{s p}}-\mathbf{P}_{\mathrm{ev}}}{\mathbf{R}_{\mathbf{e p}}}-\frac{\mathbf{P}_{\mathrm{ev}}-\mathbf{P}_{\mathrm{AD}}}{\mathbf{R}_{\mathrm{ev}}}-\frac{\mathbf{d V} \mathbf{V}_{\mathrm{u}, \mathrm{ev}}}{\mathbf{d t}}
$$

onde o índice $\mathbf{A D}$ representa o átrio direito e $\mathbf{V}_{\mathbf{u}, \mathbf{e v}}$ é o volume não distendido. Note que $\mathbf{V}_{\mathbf{u}, \mathbf{e v}}$ é uma variável, um atuador do mecanismo de reflexo baroceptor, e não uma constante ou valor inicial; sua variação será descrita mais adiante.

A circulação esplâncnica venosa é obtida a partir dos demais valores já determinados, ficando:

$$
\begin{aligned}
C_{s v} P_{s v}= & V_{t}-C_{s a} P_{s a}-\left(C_{s p}+C_{e p}\right) P_{s p}-C_{e v} P_{e v}-C_{A D} P_{A D}-V_{v D} \\
& -C_{p a} P_{p a}-C_{p p} P_{p p}-C_{p v} P_{p v}-C_{A D} P_{A D}-V_{V E}-V_{u}
\end{aligned}
$$

sendo $\mathbf{V}_{\mathbf{t}}$ o volume total de sangue contido no sistema circulatório, $\mathbf{V}_{\mathbf{V D}}$ e $\mathbf{V}_{\mathbf{V E}}$ os volumes contidos respectivamente nos ventrículos direito e esquerdo, e $\mathbf{V}_{\mathbf{u}}$ o volume não distendido total, igual à soma dos volumes não distendidos dos demais compartimentos:

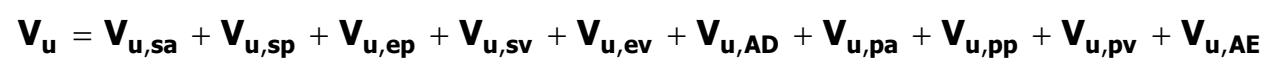

considerando que $\mathbf{A D}$ e $\mathbf{A E}$ representam os átrios direito e esquerdo, respectivamente. 
O volume total $\mathbf{V}_{\mathbf{t}}$ pode variar durante a simulação, devido a hemorragias ou infusões de sangue. Isso leva a uma nova equação diferencial:

$$
\frac{\mathbf{d V} \mathbf{t}(\mathbf{t})}{\mathbf{d t}}=\mathbf{I}(\mathbf{t})
$$

com $\mathbf{V}_{\mathbf{t}}(0)=\mathbf{V}_{\mathbf{t}, 0}$ (igual a $5300 \mathrm{ml}$, conforme a Tabela 3-1 abaixo) sendo o volume de sangue inicialmente contido na circulação. $\mathbf{I}(\mathbf{t})$ é a taxa de injeção (quando positiva) ou retirada (quando negativa) de sangue.

\section{Parâmetros}

Os parâmetros para a circulação vascular foram diretamente extraídos do trabalho de Ursino (1998), e são resumidos na Tabela 3-1 abaixo.

Tabela 3-1 Parâmetros referentes à circulação vascular.

\begin{tabular}{c|c|c|c|}
$\begin{array}{c}\text { Resistência } \\
(\mathrm{mmHg} \cdot \mathrm{s} / \mathrm{ml})\end{array}$ & $\begin{array}{c}\text { Complacência } \\
(\mathrm{ml} / \mathrm{mmHg})\end{array}$ & $\begin{array}{c}\text { Volume não } \\
\text { tensionado } \\
(\mathrm{ml})\end{array}$ & $\begin{array}{c}\text { Inertância } \\
\left(\mathrm{mmHg} \cdot \mathrm{ml} / \mathrm{s}^{2}\right)\end{array}$ \\
\hline
\end{tabular}

\begin{tabular}{|c|c|c|c|c|}
\hline 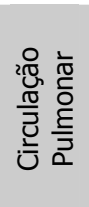 & $\begin{array}{l}\mathbf{R}_{\mathbf{p a}}=0,023 \\
\mathbf{R}_{\mathbf{p p}}=0,0894 \\
\mathbf{R}_{\mathbf{p v}}=0,0056\end{array}$ & $\begin{array}{l}\mathbf{C}_{\mathbf{p a}}=0,76 \\
\mathbf{C}_{\mathbf{p p}}=5,80 \\
\mathbf{C}_{\mathbf{p v}}=25,37\end{array}$ & $\begin{array}{l}\mathbf{V}_{\mathbf{u}, \mathbf{p a}}=0 \\
\mathbf{v}_{\mathbf{u}, \mathbf{p p}}=123 \\
\mathbf{V}_{\mathbf{u}, \mathbf{p v}}=120\end{array}$ & $\mathbf{L}_{\mathbf{p a}}=0,18 \cdot 10^{-3}$ \\
\hline 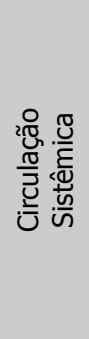 & $\begin{array}{l}\mathbf{R}_{\text {sa }}=0,06 \\
\mathbf{R}_{\text {sp }}=3,307 \\
\mathbf{R}_{\text {ep }}=1,407 \\
\mathbf{R}_{\text {sv }}=0,038 \\
\mathbf{R}_{\text {ev }}=0,016\end{array}$ & $\begin{array}{l}\mathbf{C}_{\text {sa }}=0,28 \\
\mathbf{C}_{\text {sp }}=2,05 \\
\mathbf{C}_{\text {ep }}=1,67 \\
\mathbf{C}_{\mathbf{s v}}=61,11 \\
\mathbf{C}_{\text {ev }}=50,0\end{array}$ & $\begin{array}{l}\mathbf{v}_{\mathbf{u}, \mathbf{s a}}=0 \\
\mathbf{v}_{\mathbf{u}, \mathbf{s p}}=274,4 \\
\mathbf{v}_{\mathbf{u}, \mathbf{e p}}=336,6 \\
\mathbf{v}_{\mathbf{u}, \mathbf{s v}}=1,121 \\
\mathbf{v}_{\mathbf{u}, \mathbf{e v}}=1,375\end{array}$ & $\mathbf{L}_{\mathbf{s a}}=0,22 \cdot 10^{-3}$ \\
\hline
\end{tabular}

Os valores de complacência e inertância para as grandes artérias sistêmicas e pulmonares foram extraídos de Beneken e De Wit ${ }^{1}$ (1967 apud URSINO, 1998). As resistências arteriais sistêmicas e pulmonares foram definidas para reproduzir a impedância aórtica e pulmonar na faixa de médias fre-

${ }^{1}$ BENEKEN, J. E. W.; DE WIT, B. A physical approach to hemodynamic aspects of the human cardiovascular system. In: REEVE, E. B.; GUYTON, A. C. (Eds.). Physical bases of circulatory transport: Regulation and exchange. Philadelphia: Saunders, p. 1-45, 1967. 
qüências, conforme proposto por Milnor ${ }^{2}$ (1982 apud URSINO, 1998). Os valores de resistência, complacência e volume não distendido para os compartimentos periféricos e venosos foram extraídos de Ursino, Antonucci e Belardinelli (1994), com modificações para as circulações esplâncnica e extraesplâncnica. Estes valores foram ajustados em função das porcentagens do débito cardíaco que seguem para cada ramo, com base nos trabalhos de Donald ${ }^{3}$ (1983), Greenway e Lister ${ }^{4}$ (1974) apud Ursino (1998) e Rothe (1983).

\subsubsection{O Coração}

Cada lado do coração é igualmente composto por dois compartimentos, representando os átrios e os ventrículos; as diferenças entre os dois lados existem apenas através dos valores dos parâmetros que os caracterizam.

\section{Lado Esquerdo}

Os átrios foram inicialmente considerados como elementos passivos, sem atividade contrátil ${ }^{5}$. Foram caracterizados por capacitores lineares, descritos por valores constantes de complacência e volume não distendido. O sangue passa dos átrios para os ventrículos através das válvulas atrioventriculares (válvula mitral para o lado esquerdo e tricúspide para o lado direito), representadas como resistências constantes colocadas em série com válvulas unidirecionais ideais.

A conservação de massa aplicada ao átrio esquerdo (subscrito $\mathbf{A E}$ ) fornece:

$$
\mathbf{C}_{\mathrm{AE}} \frac{\mathbf{d P _ { \mathrm { AE } }}}{\mathbf{d t}}=\frac{\mathbf{P}_{\mathrm{pv}}-\mathbf{P}_{\mathrm{AE}}}{\mathbf{R}_{\mathrm{pv}}}-\dot{\mathbf{Q}}_{\mathrm{esq}, \mathrm{e}}
$$

\footnotetext{
${ }^{2}$ MILNOR, W. R. Hemodynamics. Baltimore: Williams \& Wilkins, 1982.

${ }^{3}$ DONALD, D. E. Splanchnic circulation. In: Handbook of physiology. The cardiovascular system: Peripheral circulation and organ blood flow. Bethesda: American Physiology Society, 1983. Sec. 2, v. III, pt. 1, cap. 7, p. 219-240.

${ }^{4}$ GREENWAY, C. V.; LISTER, G. E. Capacitance effects and blood reservoir function in the splanchnic vascular bed during nonhypotensive haemorrhage and blood volume expansion in anaesthetized cats. Canadian Journal of Physiology and Pharmacology, v. 237, p. 279-294, 1974.

${ }^{5}$ Conforme mencionado na introdução deste capítulo, posteriormente será descrita a adição da contratilidade dos átrios, desenvolvida após a observação do formato das curvas de pressão atrial obtidas através das simulações do modelo original aqui descrito.
} 
sendo $\dot{\mathbf{Q}}_{\text {esq,e }}$ o fluxo de entrada no ventrículo esquerdo. O fluxo de entrada no átrio esquerdo $\dot{\mathbf{Q}}_{\mathbf{A E}}$ é dado pelo primeiro termo do lado direito da Equação 3-15, ou seja,

$$
\dot{\mathbf{Q}}_{\mathbf{A E}}=\frac{\mathbf{P}_{\mathrm{pv}}-\mathbf{P}_{\mathrm{AE}}}{\mathbf{R}_{\mathrm{pv}}}
$$

O fluxo de entrada no ventrículo esquerdo, $\dot{\mathbf{Q}}_{\mathbf{e s q}, \mathbf{e}}$, depende da abertura da válvula mitral, e pode ser descrito por

$$
\dot{\mathbf{Q}}_{\text {esq,e }}= \begin{cases}0 & \text { se } \mathbf{P}_{\mathrm{AE}} \leq \mathbf{P}_{\mathrm{VE}} \\ \frac{\mathbf{P}_{\mathrm{AE}}-\mathbf{P}_{\mathrm{VE}}}{\mathbf{R}_{\mathrm{AE}}} & \text { se } \mathbf{P}_{\mathrm{AE}}>\mathbf{P}_{\mathrm{VE}}\end{cases}
$$

Lembrando que $\mathbf{Q}_{\text {esq,s }}$ é o débito cardíaco (vazão de saída) do ventrículo esquerdo, pode-se escrever a equação da conservação de massa de modo a se obter o volume no ventrículo esquerdo $\mathbf{V}_{\mathbf{V E}}$ :

$$
\frac{\mathbf{d V} \mathbf{V}_{\mathbf{V E}}}{\mathbf{d t}}=\dot{\mathbf{Q}}_{\text {esq,e }}-\dot{\mathbf{Q}}_{\text {esq, s }}
$$

Antes de determinar $\dot{\mathbf{Q}}_{\mathbf{e s q}, \mathbf{s}}$, algumas considerações precisam ser feitas. Para descrever a atividade contrátil do ventrículo, foi utilizado um arranjo em série de uma elastância variável (que contabiliza a relação isométrica entre pressão e volume; a elastância varia durante o ciclo cardíaco em função da atividade contrátil do ventrículo) e uma resistência variável (que reflete a resistência viscosa do ventrículo à passagem do sangue; a resistência aumenta com a atividade contrátil das fibras musculares), conforme descrito por Schroff, Janicki e Weber ${ }^{6}$ (1985 apud URSINO, 1998). Ursino utilizou a alça de pressão-volume do ventrículo (ver Figura 3-3) para obter a função de elasticidade. O enchimento ventricular, durante a diástole (fase I da Figura 3-3b), obedece a uma função exponencial (GUYTON, 1997), que reflete a elasticidade do músculo relaxado e de suas restrições externas (linha tracejada a na Figura 3-3b). A função pressão-volume no fim da sístole foi adotada como linear por Ursino (linha tracejada $b$ na Figura 3-3b), seguindo o trabalho de Sagawa7 (1988 apud URSINO, 1998); a tangente desta função fornece a elasticidade sistólica final, denotada $\mathbf{E}_{\max }$.

\footnotetext{
${ }^{6}$ SCHROFF, S. G.; JANICKI, J. S.; WEBER, K. T. Evidence and quantitation of left ventricular systolic resistance. American Journal of Physiology: Heart and Circulatory Physiology, v. 249, p. H358-H370, 1985.

${ }^{7}$ SAGAWA, K. W.; MAUGHAN, H.; SUGA, H.; SUNAGAWA, K. Cardiac contraction and the pressure-volume relationship. New York: Oxford University Press, 1988.
} 


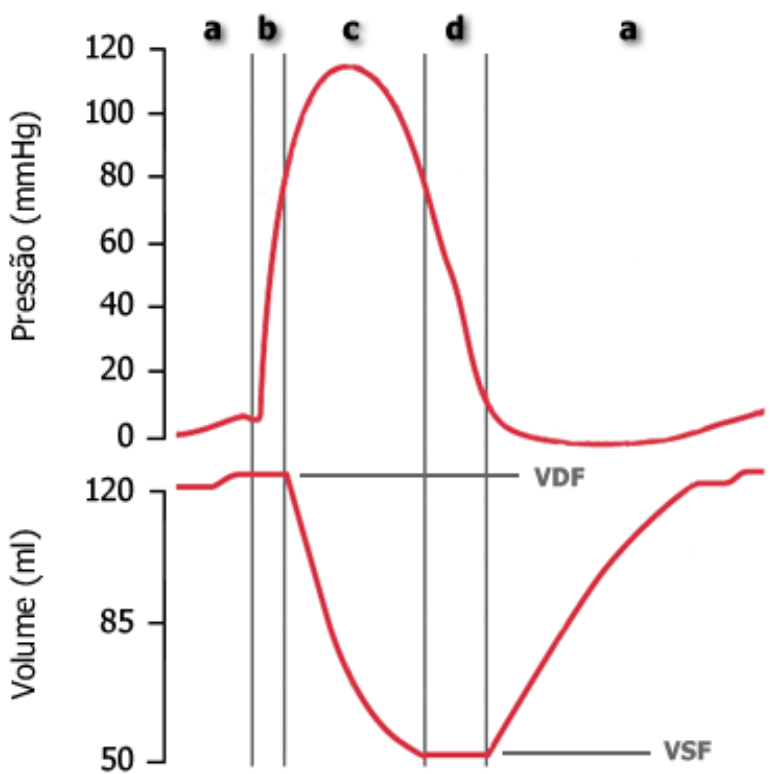

(a)

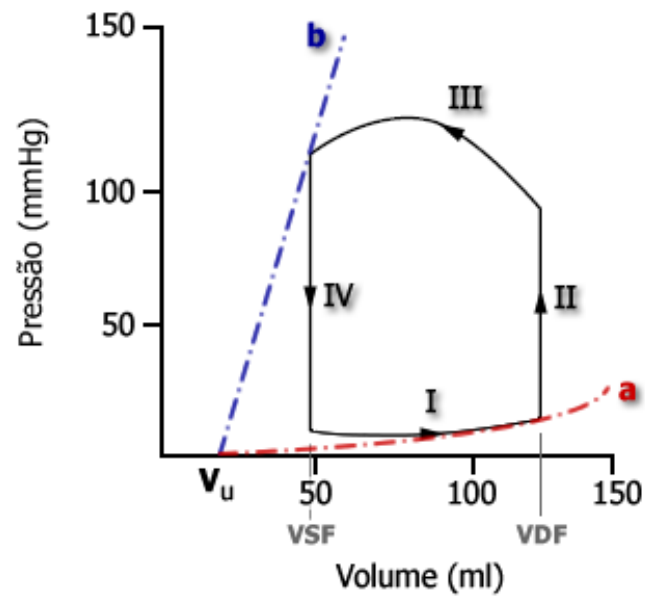

(b)

Figura 3-3 Relação pressão-volume do ventrículo esquerdo. A alça pressão-volume exibida em (b) é obtida a partir das variações de pressão e volume em um ciclo cardíaco, exibido em (a). As fases do ciclo estão numeradas em algarismos romanos: I. fase de enchimento, II. contração isovolumétrica, III. fase de ejeção, IV. relaxamento isovolúmico - note as fases isovolumétricas em (a) e (b), representadas pelo volume diastólico final (VDF) e volume sistólico final (VSF). As linhas tracejadas em (b) marcadas como $a$ (em vermelho) e $b$ (em azul) representam as funções pressão-volume na diástole e no fim da sístole, respectivamente (adaptado de Rhoades, 2003, com base em Ursino, 1998 e Guyton, 1997).

A transição entre sístole e diástole é governada por uma função de ativação pulsante $\varphi(\mathbf{t})$, com período $\mathbf{T}$ igual ao período cardíaco (que corresponde ao inverso da freqüência cardíaca). Ursino propõe a utilização de uma senóide quadrada como expressão para $\varphi(\mathbf{t})$, conforme proposto por Piene ${ }^{8}$ (1984 apud URSINO, 1998) - a função de ativação pode ser visualizada na Figura 3-4. Além disso, o período cardíaco varia em função das ações de controle do barorreflexo, conforme será descrito mais adiante, e segundo Weissler, Harris e Schoenfeld ${ }^{9}$ (1968 apud URSINO, 1998), a duração da sístole decai linearmente com a freqüência cardíaca.

\footnotetext{
${ }^{8}$ PIENE, H. Impedance matching between ventricle and load. Annals of Biomedical Engineering, v. 12, p. 191-207, 1984.

${ }^{9}$ WEISSLER, A. M.; HARRIS, W. S.; SCHOENFELD, C. D. Systolic time intervals in heart failure in man. Circulation, v. 37, p.149-159, 1968.
} 


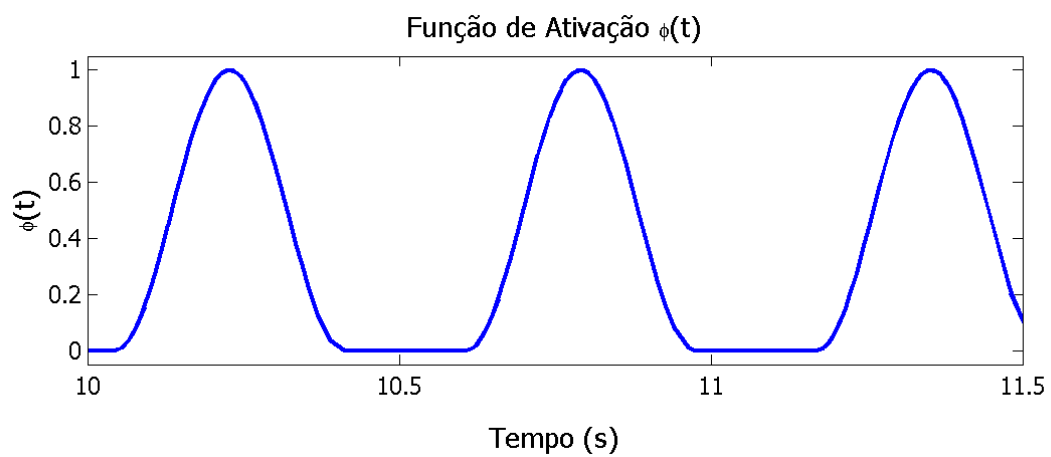

Figura 3-4 Função de ativação do ventrículo $\varphi(t)$.

Agora, pode-se prosseguir na descrição de $\dot{\mathbf{Q}}_{\mathbf{e s q}, \mathbf{s}}$, que depende da abertura da válvula aórtica, considerada ideal:

$$
\dot{\mathbf{Q}}_{\text {esq }, \mathbf{s}}= \begin{cases}0 & \text { se } \mathbf{P}_{\max , \mathbf{V E}} \leq \mathbf{P}_{\mathbf{s a}} \\ \frac{\mathbf{P}_{\max , \mathbf{V E}}-\mathbf{P}_{\mathbf{s a}}}{\mathbf{R}_{\mathbf{V E}}} & \text { se } \mathbf{P}_{\max , \mathbf{V E}}>\mathbf{P}_{\mathbf{s a}}\end{cases}
$$

onde $\mathbf{P}_{\max , \mathbf{V E}}$ é a pressão isométrica do ventrículo esquerdo (função de elasticidade) e $\mathbf{R}_{\mathbf{V E}}$ é a respectiva resistência viscosa. Conforme citado, assume-se que $\mathbf{R}_{\mathbf{V E}}$ seja proporcional à pressão isométrica $\mathbf{P}_{\max , \mathbf{V E}}$, crescendo significativamente durante a contração:

$$
\mathbf{R}_{\mathbf{V E}}=\mathbf{k}_{\mathbf{R}, \mathbf{V E}} \mathbf{P}_{\max , \mathbf{V E}}
$$

sendo $\mathbf{k}_{\mathbf{R}, \mathbf{v E}}$ uma constante de proporcionalidade. Desta forma, pode-se afirmar que a pressão instantânea no ventrículo esquerdo $\mathbf{P}_{\mathbf{V E}}$ consista na diferença entre a pressão isométrica e as perdas viscosas (contabilizando, assim, os efeitos da contratilidade do músculo e da resistência à passagem do sangue), ou seja,

$$
\mathbf{P}_{\mathbf{V E}}=\mathbf{P}_{\mathrm{max}, \mathbf{V E}}-\mathbf{R}_{\mathbf{V E}} \dot{\mathbf{Q}}_{\mathbf{e s q}, \mathbf{s}}
$$

A função pressão-volume pode ser finalmente escrita, baseada nas considerações mencionadas anteriormente (exponencial na diástole, com o ventrículo relaxado, e linear na sístole, com o ventrículo contraído em seu máximo):

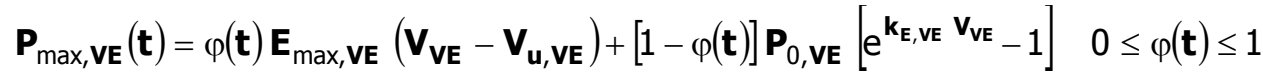

onde $\mathbf{E}_{\max , \mathbf{V E}}$ é a elastância do ventrículo no momento de contração máxima, $\mathbf{V}_{\mathbf{u}, \mathbf{V E}}$ é o volume não distendido, correspondente ao ponto em que a função pressão-volume diastólico final cruza o eixo $x$, e $\mathbf{P}_{0, \mathbf{V E}}$ e $\mathbf{k}_{\mathbf{E}, \mathbf{V E}}$ são constantes que caracterizam a função pressão-volume exponencial na diástole. 
A função de ativação do ventrículo $\varphi(\mathbf{t})$, conforme citado, consiste em uma senóide quadrada, com valor igual a 1 em contração máxima e 0 em relaxamento total,

$$
\varphi(\mathbf{t})= \begin{cases}\operatorname{sen}^{2}\left[\frac{\pi \mathbf{T}(\mathbf{t})}{\mathbf{T}_{\text {sis }}(\mathbf{t})} \cdot \mathbf{u}\right] & 0 \leq \mathbf{u} \leq \frac{\mathbf{T}_{\text {sis }}}{\mathbf{T}} \\ 0 & \frac{\mathbf{T}_{\text {sis }}}{\mathbf{T}} \leq \mathbf{u} \leq 1\end{cases}
$$

sendo $\mathbf{T}$ o período cardíaco (inverso da freqüência cardíaca), $\mathbf{T}_{\text {sis }}$ a duração da sístole, e $\mathbf{u}$ um adimensional que varia entre 0 e 1, representando a fração do ciclo cardíaco. 0 valor $\mathbf{u}=0$ foi convenientemente ajustado para o início da sístole, e a expressão para $\mathbf{u}(\mathbf{t})$ foi obtida em função de $\mathbf{T}$,

$$
\mathbf{u}(\mathbf{t})=\operatorname{rem}\left[\int_{\mathbf{t}_{0}}^{\mathbf{t}} \frac{1}{\mathbf{T}(\tau)} \mathbf{d} \tau+\mathbf{u}\left(\mathbf{t}_{0}\right)\right]
$$

onde rem corresponde à função remainder do Simulink, onde o modelo foi simulado; esta função considera apenas a parte fracionária do argumento, resultando no efeito de zerar a variável assim que ela chega ao valor 1. A Figura 3-5 abaixo exibe a função de disparo $\mathbf{u}(\mathbf{t})$ juntamente com a função de ativação $\varphi(\mathbf{t})$.
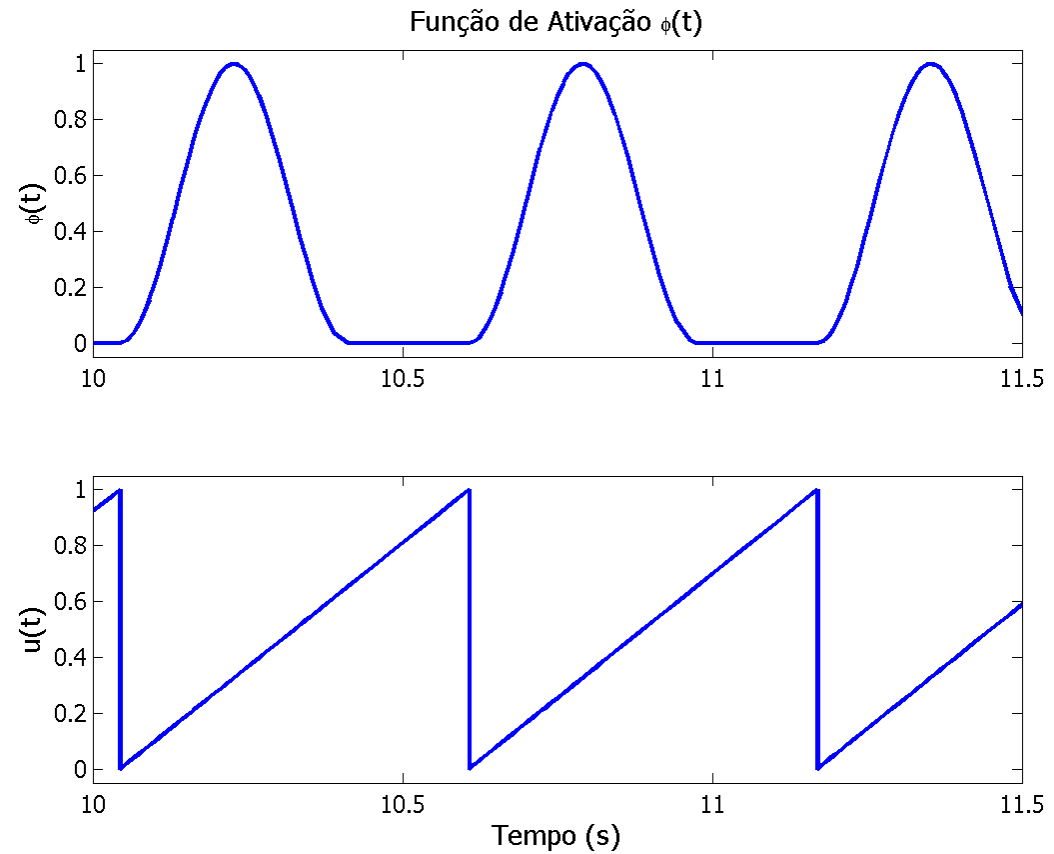

Figura 3-5 Função de ativação do ventrículo $\varphi(t)$ e função de disparo $u(t)$. Note que o ciclo em $\varphi(t)$ inicia quando $u(t)$ passa para zero.

Já foi mencionado que a duração da sístole $\mathbf{T}_{\text {sis }}$ decai linearmente com a freqüência cardíaca. 


$$
\mathbf{T}_{\text {sis }}=\mathbf{T}_{\text {sis }, 0}-\mathbf{k}_{\text {sis }} \frac{1}{\mathbf{T}}
$$

sendo $\mathbf{T}_{\text {sis }, 0}$ e $\mathbf{k}_{\text {sis }}$ parâmetros constantes.

\section{Lado Direito}

Conforme mencionado, as diferenças entre os lados esquerdo e direito estão apenas nos valores dos parâmetros. Desta forma, aplicando a conservação de massa ao átrio direito, temos

$$
\mathbf{C}_{\mathbf{A D}} \frac{\mathbf{d P}_{\mathbf{A D}}}{\mathbf{d t}}=\frac{\mathbf{P}_{\mathbf{S v}}-\mathbf{P}_{\mathrm{AD}}}{\mathbf{R}_{\mathbf{s v}}}+\frac{\mathbf{P}_{\mathrm{ev}}-\mathbf{P}_{\mathrm{AD}}}{\mathbf{R}_{\mathrm{ev}}}-\dot{\mathbf{Q}}_{\mathrm{dir}, \mathbf{e}}
$$

com $\dot{\mathbf{Q}}_{\text {dir,e }}$ sendo a vazão de entrada no ventrículo direito. O fluxo de entrada no átrio direito $\dot{\mathbf{Q}}_{\mathbf{A D}}$ pode ser determinado através da soma dos dois primeiros termos da Equação 3-26, ou seja,

$$
\dot{\mathbf{Q}}_{\mathrm{AD}}=\frac{\mathbf{P}_{\mathbf{s v}}-\mathbf{P}_{\mathrm{AD}}}{\mathbf{R}_{\mathbf{s v}}}+\frac{\mathbf{P}_{\mathrm{ev}}-\mathbf{P}_{\mathbf{A D}}}{\mathbf{R}_{\mathrm{ev}}}
$$

A vazão de entrada no ventrículo direito $\dot{\mathbf{Q}}_{\mathrm{dir}, \mathbf{e}}$ fica,

$$
\dot{\mathbf{Q}}_{\text {dir }, \mathbf{e}}= \begin{cases}0 & \text { se } \mathbf{P}_{\mathrm{AD}} \leq \mathbf{P}_{\mathrm{VD}} \\ \frac{\mathbf{P}_{\mathrm{AD}}-\mathbf{P}_{\mathrm{VD}}}{\mathbf{R}_{\mathrm{AD}}} & \text { se } \mathbf{P}_{\mathrm{AD}}>\mathbf{P}_{\mathrm{VD}}\end{cases}
$$

Sendo $\dot{\mathbf{Q}}_{\text {dir,s }}$ o débito cardíaco (fluxo de saída) do ventrículo direito, a equação de conservação de massa fornece $\mathbf{V}_{\mathbf{V D}}$, o volume no ventrículo direito:

$$
\frac{\mathbf{d V} \mathbf{V}_{\mathrm{VD}}}{\mathbf{d t}}=\dot{\mathbf{Q}}_{\mathrm{dir}, \mathrm{e}}-\dot{\mathbf{Q}}_{\mathrm{dir}, \mathbf{s}}
$$

O débito cardíaco do ventrículo direito pode ser escrito como (valendo-se das mesmas considerações feitas para o lado esquerdo):

$$
\dot{\mathbf{Q}}_{\text {dir }, \mathbf{s}}= \begin{cases}0 & \text { se } \mathbf{P}_{\max , \mathbf{v D}} \leq \mathbf{P}_{\mathbf{p a}} \\ \frac{\mathbf{P}_{\max , \mathbf{V D}}-\mathbf{P}_{\mathbf{p a}}}{\mathbf{R}_{\mathbf{V D}}} & \text { se } \mathbf{P}_{\max , \mathbf{v D}}>\mathbf{P}_{\mathbf{p a}}\end{cases}
$$

A resistência viscosa do ventrículo direito $\mathbf{R}_{\mathbf{V D}}$ fica:

$$
\mathbf{R}_{\mathbf{V D}}=\mathbf{k}_{\mathbf{R}, \mathbf{v D}} \mathbf{P}_{\text {max }, \mathbf{V D}}
$$


sendo $\mathbf{k}_{\mathbf{R}, \mathbf{v D}}$ a constante de proporcionalidade para o lado direito. A pressão instantânea no ventrículo $\mathbf{P}_{\mathbf{v D}}$ pode ser obtida por

$\mathbf{P}_{\mathrm{VD}}=\mathbf{P}_{\mathrm{max}, \mathbf{V D}}-\mathbf{R}_{\mathrm{VD}} \dot{\mathbf{Q}}_{\mathrm{dir}, \mathbf{s}}$

Finalmente, a relação pressão volume é dada por

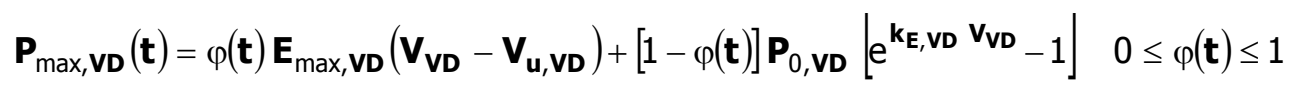

A função de ativação do ventrículo $\varphi(\mathbf{t})$, descrita pelas Equações 3-23 e 3-24, é a mesma para ambos os lados.

\section{Parâmetros}

Novamente, os parâmetros foram extraídos do trabalho de Ursino (1998). Os valores de complacência e volumes não distendidos para os átrios direito e esquerdo e de resistência para as válvulas tricúspide e mitral foram ajustados para atingir os padrões de tempo das pressões e volumes atriais descritos em Milnor ${ }^{10}$ (1982 apud URSINO, 1998). Os parâmetros $\mathbf{P}_{0, \mathbf{V E}}$ e $\mathbf{k}_{\mathbf{E}, \mathbf{V E}}$, que descrevem as relações pressão-volume no fim da diástole para o ventrículo esquerdo, foram extraídos de Gaasch et al. ${ }^{11}$ (1976 apud URSINO, 1998), enquanto os equivalentes para o lado direito, $\mathbf{P}_{0, \mathbf{V D}}$ e $\mathbf{k}_{\mathbf{E}, \mathbf{V D}}$, foram obtidos de Janicki e Weber ${ }^{12}$ (1980 apud URSINO, 1998). Os valores para viscosidade dos ventrículos, $\mathbf{k}_{\mathbf{R}, \mathbf{V E}}$ e $\mathbf{k}_{\mathbf{R}, \mathbf{V D}}$ foram extraídos de experimentos realizados em cachorros (CAMPBELL et al. ${ }^{13}, 1987$; SCHROFF, JANICKI e WEBER ${ }^{14}, 1985$ apud URSINO, 1998), redimensionados para refletir as diferenças entre o volume ventricular humano e o canino.

\footnotetext{
${ }^{10}$ MILNOR, W. R. Hemodynamics. Baltimore: Williams \& Wilkins, 1982.

${ }^{11}$ GAASCH, W. H.; LEVINE, H. J.; QUINONES, M. A.; ALEXANDER, J. K. Left ventricular compliance: Mechanisms and clinical implications. American Journal of Cardiology, v. 38, p. 645-653, 1976.

12 JANICKI, J. S.; WEBER, K. T. The pericardium and ventricular interaction, distensibility, and function. American Journal of Physiology: Heart and Circulatory Physiology, v. 238, p. H494-H503, 1980.

${ }^{13}$ CAMPBELL, K. B.; RINGO, J. A.; WAKAO, Y.; KLAVANO, P. A.; ALEXANDER, J. E. Internal capacitance and resistance allow prediction of right ventricle outflow. American Journal of Physiology: Heart and Circulatory Physiology, v. 243, p. H99-H112, 1982.

${ }^{14}$ SCHROFF, S. G.; JANICKI, J. S.; WEBER, K. T. Evidence and quantitation of left ventricular systolic resistance. American Journal of Physiology: Heart and Circulatory Physiology, v. 249, p. H358-H370, 1985.
} 
Os valores de $\mathbf{E}_{\max , \mathbf{V E}}$ e $\mathbf{V}_{\mathbf{u}, \mathbf{V E}}$, que descrevem a função pressão-volume no fim da sístole para o ventrículo esquerdo, foram obtidos de Asanoi, Sasayama e Kameyama ${ }^{15}$ (1989) e Takeushi et al. ${ }^{16}$ (1991) apud Ursino, 1998. Os equivalentes para o lado direito, $\mathbf{E}_{\max , \mathbf{V D}}$ e $\mathbf{V}_{\mathbf{u}, \mathbf{V D}}$, foram derivados de valores reportados para cachorros por Janicki e Weber ${ }^{17}$ (1980) e Maughan, Sunagawa e Sagawa $^{18}$ (1987) apud Ursino, 1998. Segundo Ursino, as relações entre os mencionados parâmetros para os lados direito e esquerdo sugeridas nestes trabalhos (ver Tabela 3-2) mostraram-se válidas também para humanos. Os parâmetros $\mathbf{T}_{\mathbf{s i s}, 0}$ e $\mathbf{k}_{\text {sis }}$, que fornecem a duração da sístole em função da freqüência cardíaca, bem como o valor de período cardíaco $\mathbf{T}$ em condições basais, foram extraídos de Weissler, Harris e Schoenfeld ${ }^{19}$ (1968 apud URSINO, 1998). Os valores são resumidos na Tabela 3-2 abaixo.

Tabela 3-2 Parâmetros referentes ao coração.

\begin{tabular}{|c|c|c|c|}
\hline & Coração Esquerdo & Coração Direito & \\
\hline . & $\begin{aligned} \mathbf{R}_{\mathbf{A E}} & =2,5 \cdot 10^{-3} \mathrm{mmHg} \cdot \mathrm{s} / \mathrm{ml} \\
\mathbf{C}_{\mathbf{A E}} & =19,23 \mathrm{ml} / \mathrm{mmHg} \\
\mathbf{V}_{\mathbf{u}, \mathbf{A E}} & =25 \mathrm{ml}\end{aligned}$ & $\begin{aligned} \mathbf{R}_{\mathbf{A D}} & =2,5 \cdot 10^{-3} \mathrm{mmHg} \cdot \mathrm{s} / \mathrm{ml} \\
\mathbf{C}_{\mathbf{A D}} & =31,25 \mathrm{ml} / \mathrm{mmHg} \\
\mathbf{V}_{\mathbf{u}, \mathbf{A D}} & =25 \mathrm{ml}\end{aligned}$ & \\
\hline 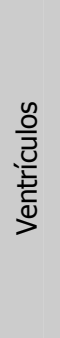 & $\begin{aligned} \mathbf{P}_{0, \mathbf{V E}} & =1,5 \mathrm{mmHg} \\
\mathbf{k}_{\mathbf{E}, \mathbf{V E}} & =0,014 \mathrm{ml}^{-1} \\
\mathbf{V}_{\mathbf{u}, \mathbf{V E}} & =16,77 \mathrm{ml} \\
\mathbf{E}_{\max , \mathbf{V E}} & =2,95 \mathrm{mmHg} / \mathrm{ml} \\
\mathbf{k}_{\mathbf{R}, \mathbf{V E}} & =3,75 \cdot 10^{-4} \mathrm{~s} / \mathrm{ml}\end{aligned}$ & $\begin{aligned} \mathbf{P}_{0, \mathbf{V D}} & =1,5 \mathrm{mmHg} \\
\mathbf{k}_{\mathbf{E}, \mathbf{V D}} & =0,011 \mathrm{ml}^{-1} \\
\mathbf{V}_{\mathbf{u}, \mathbf{V D}} & =40,8 \mathrm{ml} \\
\mathbf{E}_{\max , \mathbf{V D}} & =1,75 \mathrm{mmHg} / \mathrm{ml} \\
\mathbf{k}_{\mathbf{R}, \mathbf{V E}} & =1,4 \cdot 10^{-4} \mathrm{~s} / \mathrm{ml}\end{aligned}$ & $\begin{aligned} \mathbf{E}_{\max , \mathbf{V D}} / \mathbf{E}_{\max , \mathbf{V E}} & =0,59 \\
\mathbf{V}_{\mathbf{u}, \mathbf{V D}} / \mathbf{V}_{\mathbf{u}, \mathbf{V E}} & =2,45\end{aligned}$ \\
\hline & $\begin{aligned} \mathbf{T} & =0,833 \mathrm{~s} \\
\mathbf{k}_{\text {sis }} & =0,075 \mathrm{~s}^{2} \\
\mathbf{T}_{\text {sis }, 0} & =0,5 \mathrm{~s}\end{aligned}$ & $\mathbf{F C}=72 \mathrm{bpm}^{*}=1,2 \mathrm{~Hz}$ & \\
\hline
\end{tabular}

* bpm - batimentos por minuto

${ }^{15}$ ASANOI, H. S.; SASAYAMA, S.; KAMEYAMA, T. Ventriculoarterial coupling in normal and failing heart in humans. Circulation Research, v. 56, p. 483-493, 1989.

${ }^{16}$ TAKEUCHI, M.; IGARASHI, Y.; TOMIMOTO, S.; ODAKE, M.; HAYASHI, T.; TSUKAMOTO, T.; HATA, K.; TAKAOKA, H.; FUKUZAKI, H. Single-beat estimation of the slope of the end-systolic pressure-volume relation in the human left ventricle. Circulation, v. 83, p. 202-212, 1991.

${ }^{17}$ JANICKI, J. S.; WEBER, K. T. The pericardium and ventricular interaction, distensibility, and function. American Journal of Physiology: Heart and Circulatory Physiology, v. 238, p. H494-H503, 1980.

${ }^{18}$ MAUGHAN, W. L.; SUNAGAWA, K.; SAGAWA, K. Ventricular systolic interdependence: volume elastance model in isolated canine hearts. American Journal of Physiology: Heart and Circulatory Physiology, v. 253, p. H1381-H1390, 1987.

${ }^{19}$ WEISSLER, A. M.; HARRIS, W. S.; SCHOENFELD, C. D. Systolic time intervals in heart failure in man. Circulation, v. 37, p.149-159, 1968. 


\subsection{Sistema de Controle - O Mecanismo Barorreflexo}

O principal mecanismo de controle da pressão arterial a curto prazo é o reflexo baroceptor, ou barorreflexo, que atua constantemente para mantê-la em seu nível normal ou próximo dele (GUYTON e HALL, 1996). Este reflexo é desencadeado pelos receptores sensíveis a pressão, encontrados nas paredes do arco aórtico e do seio carotídeo (dilatação próxima à ramificação da artéria carótida comum em interna e externa), conforme Figura 3-6 abaixo (KAREMAKER, 1987). Há também sensores localizados em outras artérias e ainda nos átrios direito e esquerdo, mas em menor número e com atividade bastante reduzida em comparação aos anteriores (GUYTON e HALL, 1996; RHOADES e TANNER, 2003).

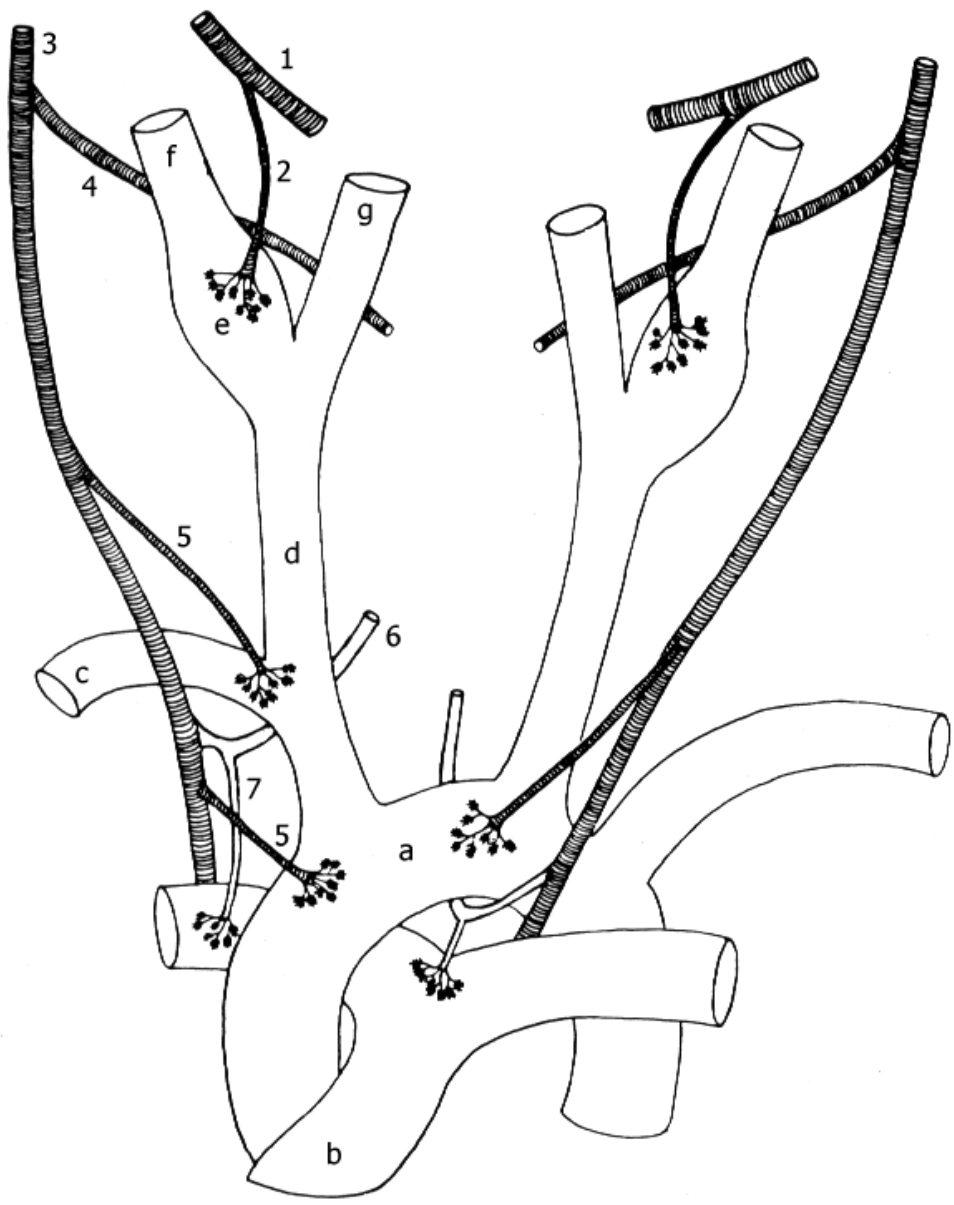

Figura 3-6 Anatomia dos vasos que possuem os mais importantes barorreceptores (vista frontal). Vasos sangüíneos: a - arco aórtico, b - tronco pulmonar, c - artéria subclávica, d - artéria carótida comum, e - seio carotídeo, $\mathrm{f}$ - artéria carótida interna, $\mathrm{g}$ - artéria carótida externa. Vias nervosas: 1 - nervo glossofaríngeo, 2 - nervo do seio carotídeo, 3 - nervo vago, 4 - nervo laríngeo superior, 5 ramos vagos cardíacos (originados no arco aórtico e suas ramificações), 6 - nervo laríngeo recorrente, 7 - ramos vagos cardíacos (originados no tronco pulmonar e suas ramificações) (adaptado de Karemaker, 1987). 
A regulação da pressão arterial é feita em decorrência das respostas transmitidas por estes receptores de pressão ao sistema nervoso central (mais especificamente, ao feixe solitário da região bulbar do tronco cerebral) através das vias aferentes representadas na Figura 3-6 acima (GUYTON e HALL, 1996). A ação dos barorreceptores se dá pela distensão das paredes arteriais devido ao aumento da pressão, fazendo com que a freqüência de impulsos nervosos aumente (RHOADES e TANNER, 2003). O inverso acontece no caso da queda de pressão, com a conseqüente contração das paredes e diminuição da freqüência dos impulsos enviados. Estas respostas são não lineares com relação à pressão, como pode ser visto na Figura 3-7 para os receptores do seio carotídeo, apresentando um valor máximo de sensibilidade (próximo ao centro da faixa de medição), com saturação superior e limiar inferior, onde a sensibilidade é reduzida ou inexistente.

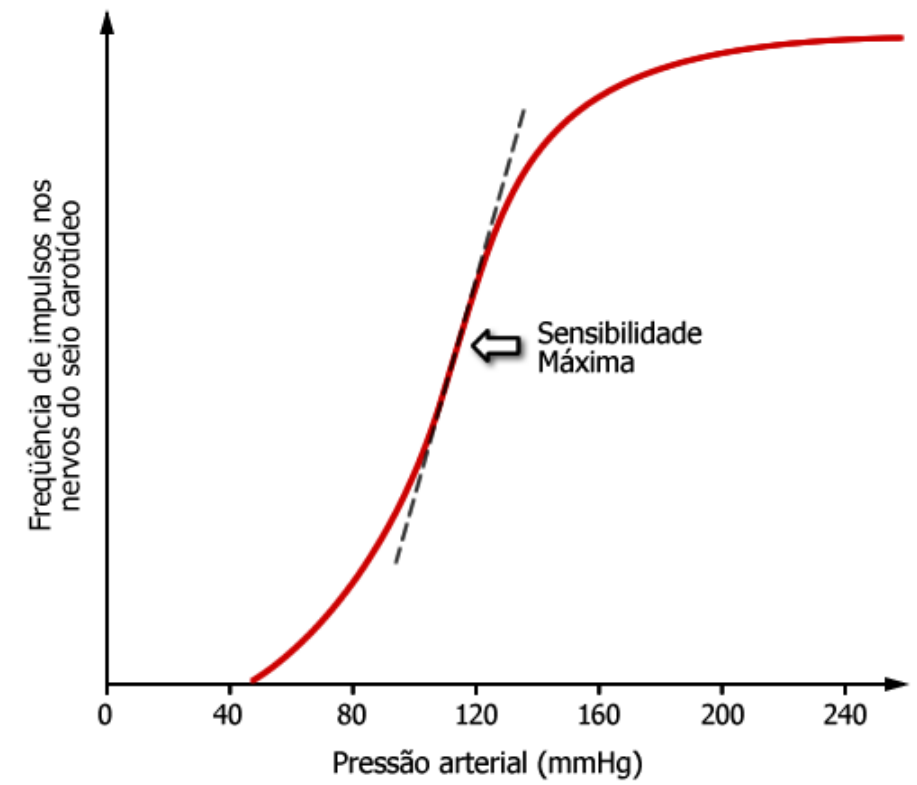

Figura 3-7 Resposta do barorreceptor carotídeo em diferentes níveis de pressão arterial (adaptado de Guyton e Hall, 1996).

A variação da freqüência de impulsos recebidos pelo feixe solitário produz alterações na atividade nervosa simpática e parassimpática (vias eferentes), que por sua vez estimulam os atuadores. Os nervos simpáticos estão relacionados com o controle da resistência periférica sistêmica, dos volumes venosos não distendidos e da contratilidade do coração (há também certa influência sobre a freqüência cardíaca). Os nervos parassimpáticos (também denominados vagos) estão relacionados com o controle da freqüência cardíaca (GUYTON e HALL, 1996). Os efeitos das alterações na freqüência de impulsos nas vias simpáticas e parassimpáticas serão discutidos nos itens seguintes. A Figura 3-8 a- 
baixo mostra o modelo hidráulico anteriormente apresentado acrescido dos elementos correspondentes ao controle pelo mecanismo barorreflexo.

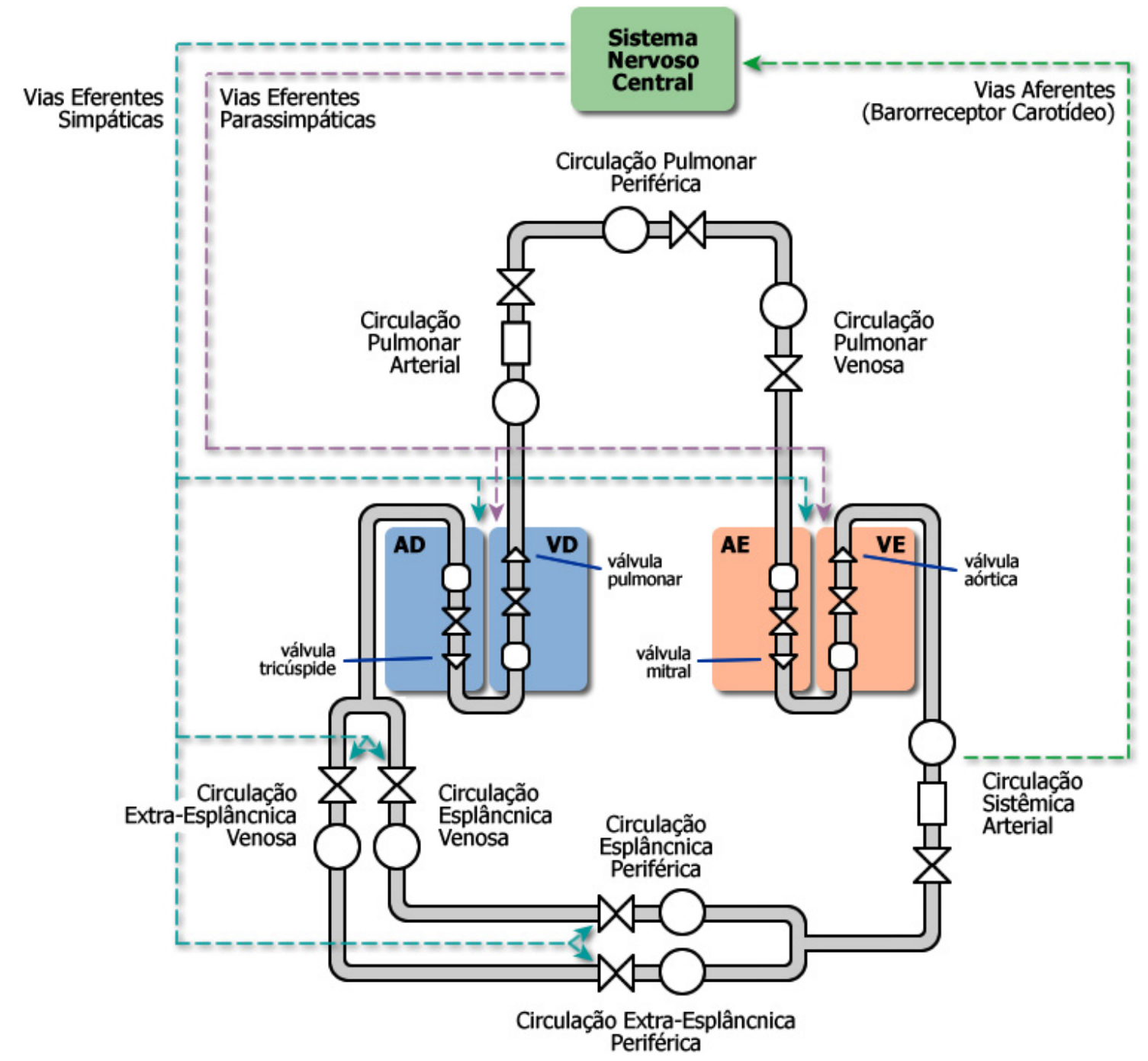

Figura 3-8 Modelo hidráulico do sistema cardiovascular com os elementos componentes do controle pelo mecanismo barorreflexo, com os caminhos nervosos mostrados através das linhas pontilhadas.

\subsubsection{Vias Aferentes}

Os receptores carotídeos respondem a níveis de pressão sangüínea variando entre 60 e 180 mmHg, apresentando sensibilidade máxima nas proximidades da pressão arterial média normal (para a maioria dos indivíduos), de cerca de $100 \mathrm{mmHg}$ (GUYTON e HALL, 1996), conforme a Figura 3-7 acima. Nesta região (destacada na Figura 3-7), mesmo a ocorrência de uma ligeira alteração de pressão produz uma forte alteração dos reflexos autonômicos (derivada máxima), reajustando a pressão arterial para os níveis normais. Os receptores aórticos não apenas respondem a níveis pelo menos 30 mmHg maiores, como também são significativamente menos sensíveis que os carotídeos (GUYTON e 
HALL, 1996; KLAUBUNDE, 2005). Por esses motivos, os receptores carotídeos são normalmente os dominantes no mecanismo barorreflexo para valores normais de pressão. Essa justificativa também vale para que apenas esses receptores sejam considerados na modelagem do sistema de controle.

Ursino utilizou resultados experimentais relatados por Chappleau e Abboud ${ }^{20}$ (1987) e Kubota et al. ${ }^{21}$ (1992) apud Ursino (1998) para descrever a relação dinâmica entre a pressão e a atividade dos nervos no seio carotídeo. Além da relação sigmóide mencionada anteriormente e exibida na Figura 3-7, esses trabalhos indicam que os barorreceptores carotídeos são sensíveis não apenas à pressão intra-seio, mas também à taxa de sua variação. Desta forma, para reproduzir estes dois efeitos na representação da sensibilidade do receptor somada à atividade dos nervos de condução, foi utilizada uma equação diferencial linear de primeira ordem (que contabiliza as características dinâmicas), cuja saída é utilizada na seqüência em uma função sigmóide estática. Assim,

$$
\begin{gathered}
\tau_{\mathbf{p}} \frac{\mathbf{d} \tilde{\mathbf{P}}}{\mathbf{d t}}+\tilde{\mathbf{P}}=\tau_{\mathbf{z}} \frac{\mathbf{d} \mathbf{P}_{\mathbf{s c}}}{\mathbf{d t}}+\mathbf{P}_{\mathbf{s c}} \\
\mathbf{f}_{\mathbf{s c}}=\frac{\mathbf{f}_{\min }+\mathbf{f}_{\max } \exp \left(\frac{\tilde{\mathbf{P}}-\mathbf{P}_{\mathbf{n}}}{\mathbf{k}_{\mathbf{a}}}\right)}{1+\exp \left(\frac{\tilde{\mathbf{P}}-\mathbf{P}_{\mathbf{n}}}{\mathbf{k}_{\mathbf{a}}}\right)}
\end{gathered}
$$

onde $\tau_{\mathbf{p}}$ e $\tau_{\mathbf{z}}$ são as constantes de tempo para o pólo e o zero reais da parte dinâmica linear (com $\left.\tau_{\mathbf{z}} / \tau_{\mathbf{p}}>1\right), \mathbf{P}_{\mathbf{s c}}$ é a pressão no seio carotídeo, $\tilde{\mathbf{P}}$ é a pressão de saída da parte linear, $\mathbf{f}_{\mathbf{s c}}$ é a freqüência de impulsos nas fibras aferentes, $\mathbf{f}_{\min }$ e $\mathbf{f}_{\max }$ são os limites de saturação respectivamente inferior e superior da freqüência de impulsos, $\mathbf{P}_{\mathbf{n}}$ é o valor da pressão correspondente ao ponto central da função sigmóide, e finalmente $\mathbf{k}_{\mathbf{a}}$ é uma constante com dimensão de pressão relacionada com a inclinação da função sigmóide no mesmo ponto central da mesma. A Figura 3-9 abaixo mostra a resposta simulada no seio carotídeo.

${ }^{20}$ CHAPLEAU, M. W.; ABBOUD, F. M. Contrasting effects of static and pulsatile pressure on carotid baroreceptor activity in dogs. Circulation Research, v. 61, p. 648-658, 1987.

${ }^{21}$ KUBOTA, T.; CHISHAKI, H.; YOSHIDA, T.; SUNAGAWA, K.; TAKESHITA, A.; NOSE, Y. How to encode arterial pressure into carotid sinus nerve to invoke natural baroreflex. American Journal of Physiology: Heart and Circulatory Physiology, v. 263, p. H307-H313, 1992. 


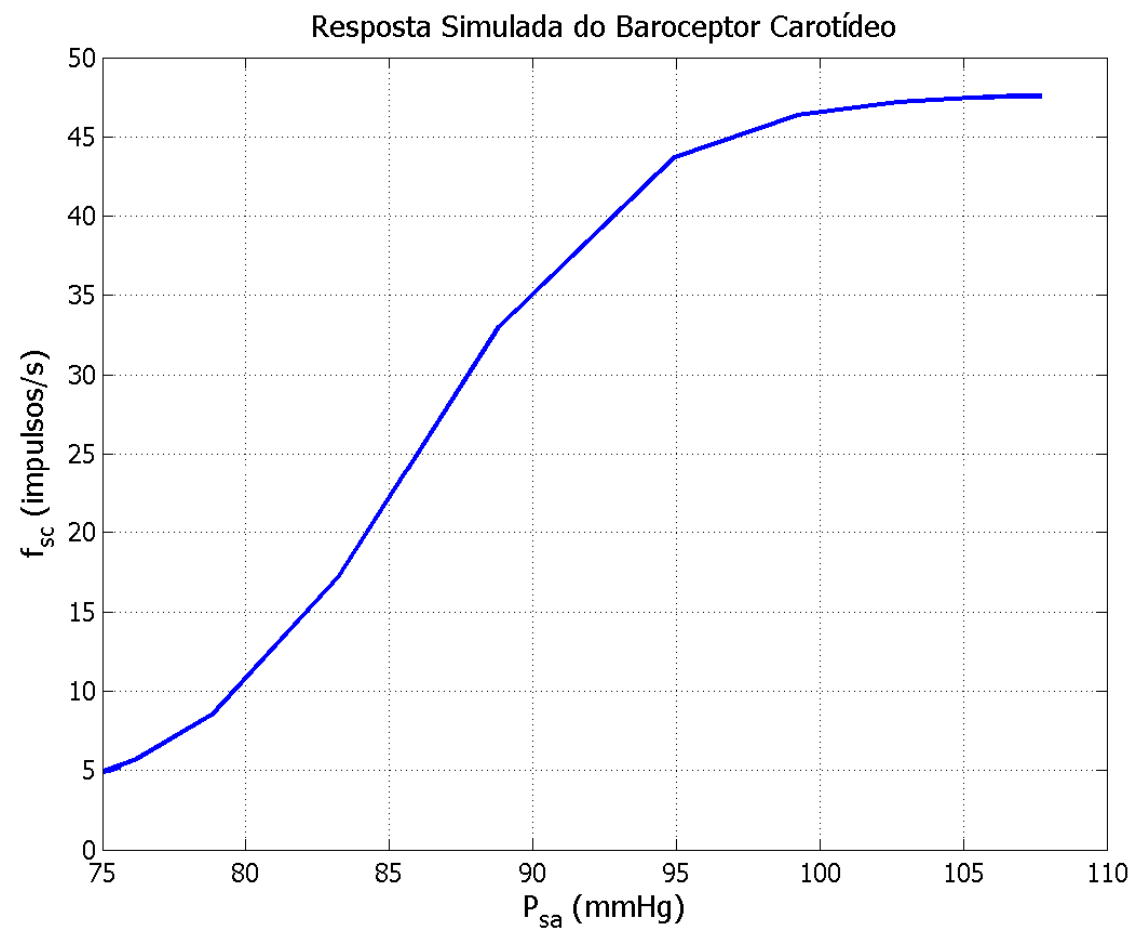

Figura 3-9 Resposta simulada do barorreceptor carotídeo.

Em condições de malha fechada, a pressão no seio carotídeo $\mathbf{P}_{\mathbf{s c}}$ fica igual à pressão sistêmica arterial $\mathbf{P}_{\mathbf{s a}}$. Em malha aberta $\mathbf{P}_{\mathbf{s c}}$ é considerada uma variável de entrada.

\section{Parâmetros}

Os parâmetros para as vias aferentes foram obtidos dos mesmos trabalhos consultados para a definição da dinâmica do barorreceptor (CHAPPLEAU e ABBOUD, 1987 e KUBOTA et al., 1992 apud URSINO, 1998), escalados para que o centro da sigmóide ficasse na em $92 \mathrm{mmHg}$. A Tabela 3-3 a seguir resume estes valores.

Tabela 3-3 Parâmetros que caracterizam a resposta produzida no seio carotídeo.

\begin{tabular}{rlrl} 
Parte Dinâmica & Parte Estática (Sigmóide) \\
$\tau_{\mathbf{z}}=6,37 \mathrm{~s}$ & $\mathbf{f}_{\min }=2,52$ & impulsos $/ \mathrm{s}$ \\
$\tau_{\mathbf{P}}=2,076 \mathrm{~s}$ & $\mathbf{f}_{\max }=47,78$ & impulsos $/ \mathrm{s}$ \\
& $\mathbf{f}_{\mathbf{s c}, 0}=25$ & impulsos $/ \mathrm{s}$ \\
$\mathbf{k}_{\mathbf{a}}$ & $=11,758$ & $\mathrm{mmHg}$ \\
$\mathbf{P}_{\mathbf{n}}$ & $=92$ & $\mathrm{mmHg}$ \\
\hline
\end{tabular}




\subsubsection{Vias Eferentes}

Os nervos simpáticos e parassimpáticos (vagos), que abundantemente inervam o coração, desempenham papel fundamental no controle da eficácia do bombeamento cardíaco, podendo tanto aumentar o débito cardíaco por mais de 100\% (através de estimulação simpática) quanto reduzi-lo a valores muito baixos (por meio de estimulação parassimpática) (GUYTON e HALL, 1996).

Conforme explicado, uma elevação na pressão arterial (média, de pulso ou ambas) resulta em um aumento na freqüência de impulsos gerados nos barorreceptores. Conseqüentemente, o sistema nervoso central provoca uma redução na estimulação simpática e um aumento na estimulação parassimpática, ambos no sentido de reduzir a pressão sangüínea até seu nível normal. A inibição do sistema nervoso simpático é capaz de diminuir o bombeamento cardíaco de forma moderada, em até 30\% abaixo de seu nível normal, através da redução da contratilidade e do aumento da resistência periférica e do volume venoso não distendido (através do mecanismo de Frank-Starling). Isso acontece porque, sob condições normais, as fibras nervosas simpáticas estão continuamente ativas, com uma freqüência suficiente para manter o débito cardíaco até $30 \%$ acima do que ocorreria sem a estimulação. Contrariamente, a estimulação do sistema parassimpático pode reduzir o débito cardíaco em mais de $50 \%$ - as inervações vagas podem interromper os batimentos cardíacos por alguns segundos (mantendo uma média mínima de 20 a 40 batimentos por segundo), além de diminuir a força de contração ventricular em até 30\% (GUYTON e HALL, 1996; RHOADES e TANNER, 2003; KLAUBUNDE, 2005). A Figura 3-10 abaixo ilustra os efeitos da elevação da pressão arterial.

Por sua vez, uma redução da pressão arterial provoca uma diminuição na freqüência de impulsos aferentes, resultando em uma resposta do sistema nervoso central que aumenta a estimulação simpática e reduz a estimulação parassimpática - agora no sentido de elevar a pressão sangüínea. Os efeitos consistem no aumento da força de contração cardíaca e a redução da resistência periférica e do volume venoso não distendido por meio da ação simpática, e um aumento da freqüência cardíaca por meio das ações parassimpática e (principalmente) simpática (GUYTON e HALL, 1996; URSINO, 1998). Os efeitos correspondem ao inverso dos mostrados na Figura 3-10 abaixo. 


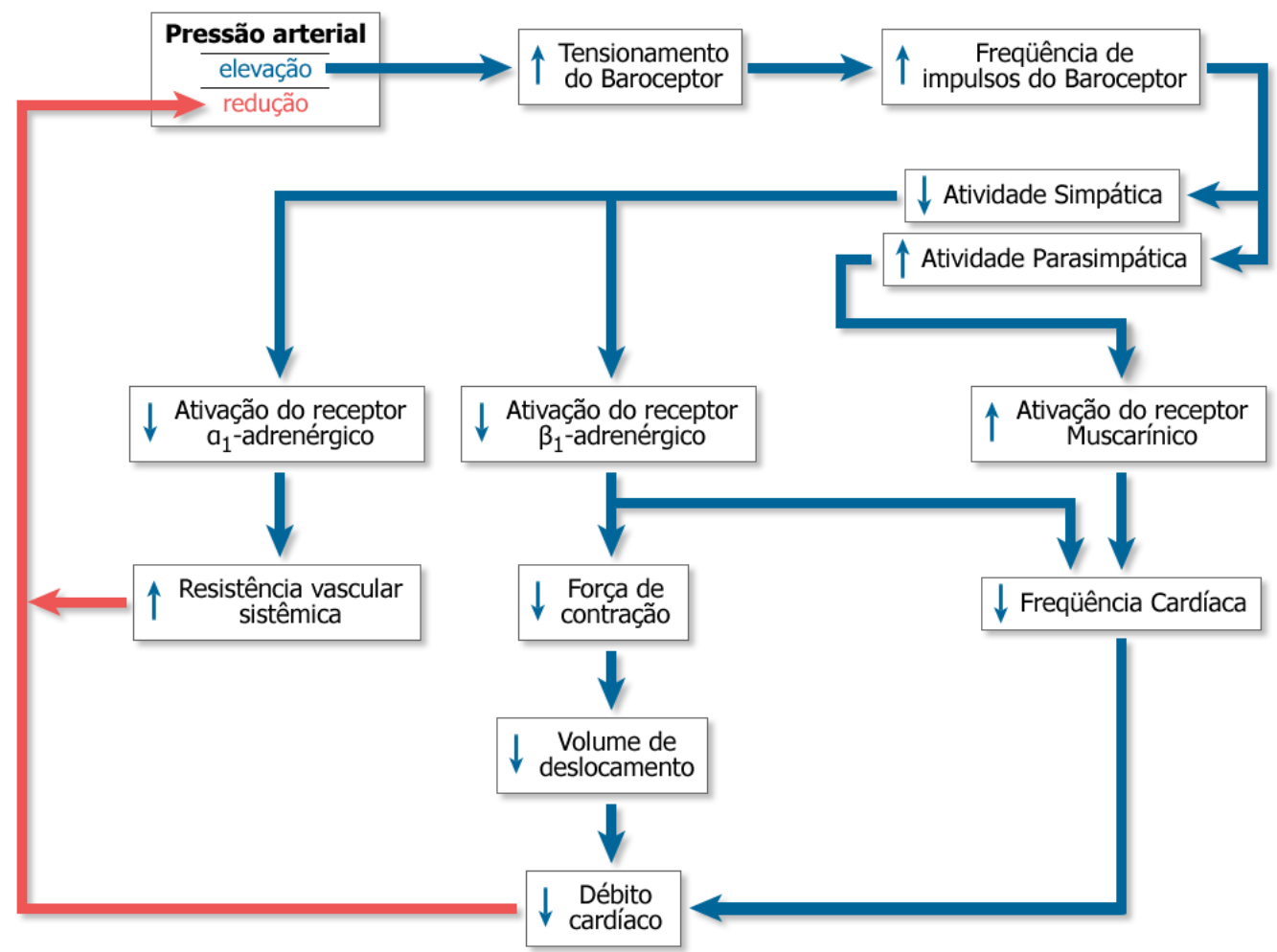

Figura 3-10 Efeitos do aumento da pressão arterial sobre as atividades simpática e parassimpática, resultando na alteração da freqüência cardíaca, da força de contração e da resistência vascular sistêmica (adaptado de Rhoades e Tanner, 2003).

Ursino reproduziu estas relações utilizando uma curva exponencial monotonicamente decrescente para a atividade simpática (cf. WANG, BRÄNDLE e ZUCKER ${ }^{22}, 1993$ apudURSINO, 1998), e uma curva exponencial monotonicamente crescente com saturação superior para a atividade parassimpática (cf. KATONA et al., 1970). Possíveis atrasos originados no sistema nervoso central não foram considerados, sendo tratados como parte da resposta dinâmica dos atuadores.

\section{Atividade Eferente Simpática}

Conforme comentado acima, pode-se escrever a expressão que descreve a atividade simpática. Define-se então a freqüência de impulsos nos nervos eferentes simpáticos, $\mathbf{f}_{\mathbf{e s}}$ :

$$
\mathbf{f}_{\mathrm{es}}=\mathbf{f}_{\mathrm{es}, \infty}+\left(\mathbf{f}_{\mathrm{es}, 0}-\mathbf{f}_{\mathrm{es}, \infty}\right) \mathbf{e}^{-\mathbf{k}_{\mathrm{es}} \mathbf{f}_{\mathrm{sc}}}
$$

${ }^{22}$ WANG, W.; BRÄNDLE, M.; ZUCKER, I. H. Influence of vagotomy on the baroreflex sensitivity in anesthetized dogs with experimental heart failure. American Journal of Physiology: Heart and Circulatory Physiology, v. 265, p. H1310H1317, 1993. 
onde $\mathbf{k}_{\mathbf{e s}}, \mathbf{f}_{\mathrm{es}, 0}$ e $\mathbf{f}_{\mathrm{es}, \infty}$ são constantes (com $\mathbf{f}_{\mathbf{e s}, 0}>\mathbf{f}_{\mathbf{e s}, \infty}$, para a curva decrescente). A Figura 3-11 abaixo mostra o formato da curva utilizada.

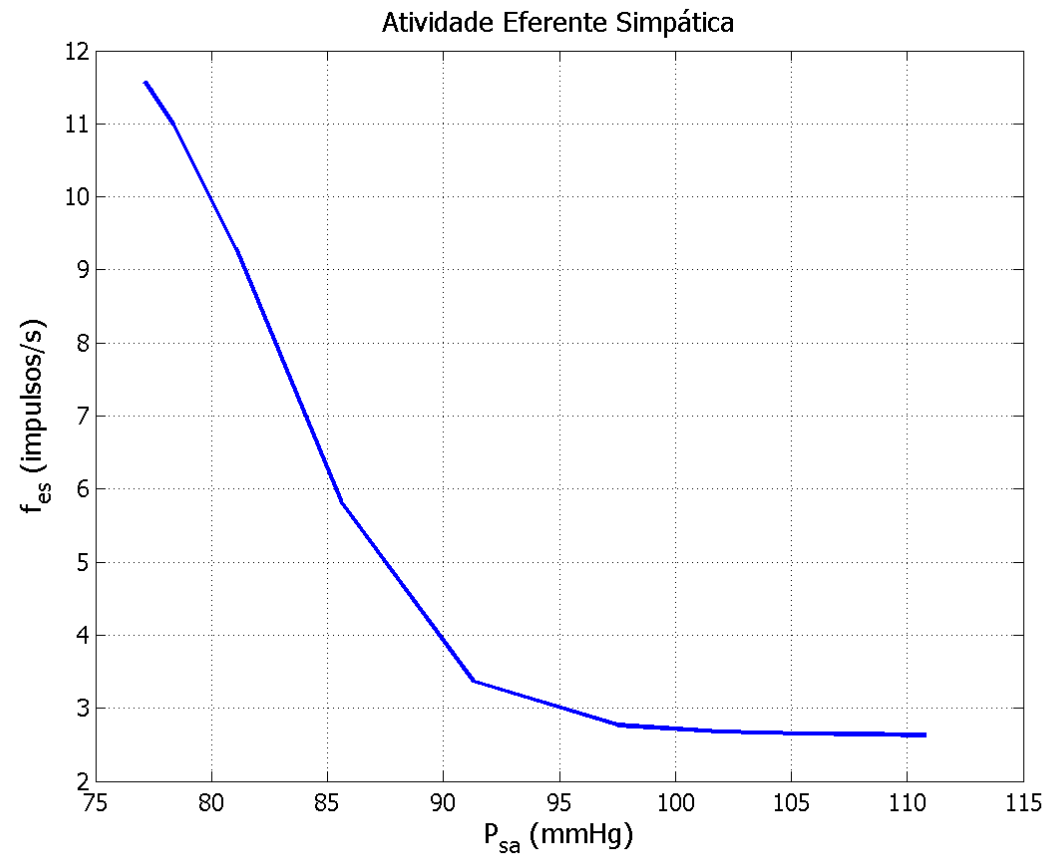

Figura 3-11 Função de atividade eferente simpática.

\section{Atividade Eferente Parassimpática (Vaga)}

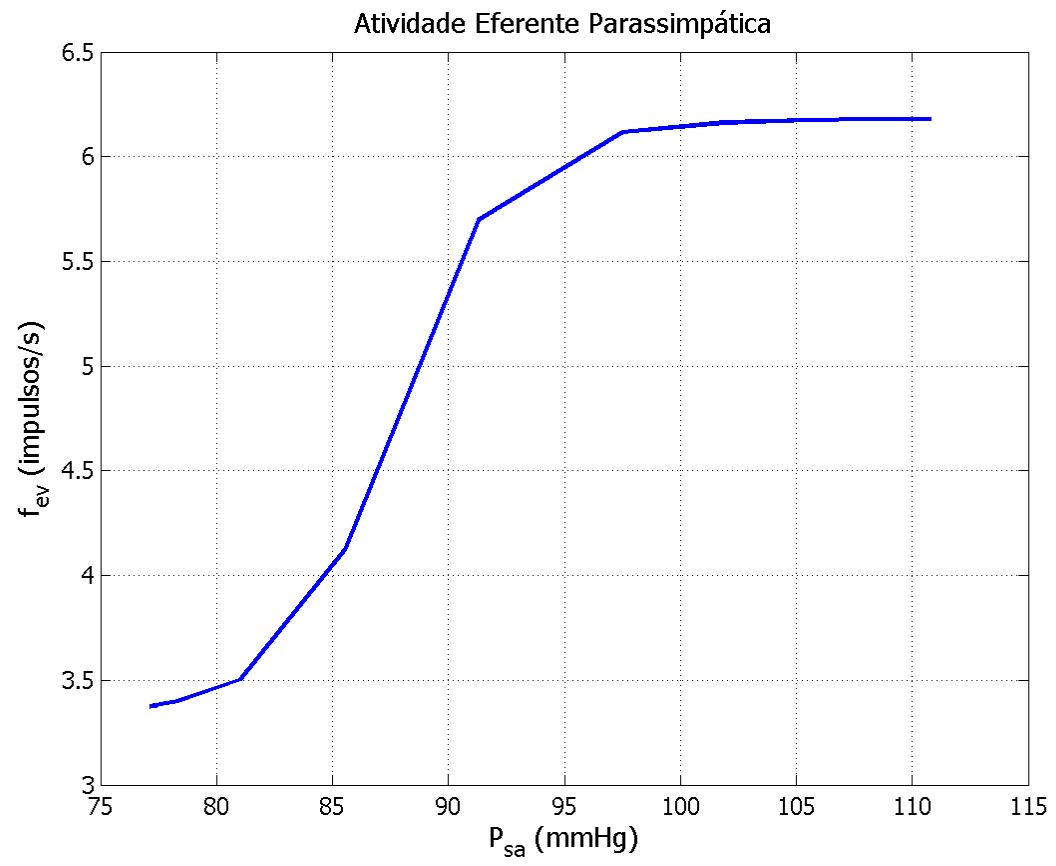

Figura 3-12 Função de atividade eferente parassimpática (vaga). 
Conforme comentado anteriormente, a atividade vaga $\mathbf{f}_{\mathrm{ev}}$ pode ser escrita com uma função monotonicamente crescente com limite superior de saturação, em uma forma sigmóide (Figura 3-12),

$$
\mathbf{f}_{\mathrm{ev}}=\frac{\mathbf{f}_{\mathrm{ev}, 0}+\mathbf{f}_{\mathrm{ev}, \infty} \exp \left(\frac{\mathbf{f}_{\mathbf{s c}}-\mathbf{f}_{\mathbf{s c}, 0}}{\mathbf{k}_{\mathrm{ev}}}\right)}{1+\exp \left(\frac{\mathbf{f}_{\mathbf{s c}}-\mathbf{f}_{\mathbf{s c}, 0}}{\mathbf{k}_{\mathrm{ev}}}\right)}
$$

onde $\mathbf{k}_{\mathrm{ev}}, \mathbf{f}_{\mathrm{ev}, 0}$ e $\mathbf{f}_{\mathrm{ev}, \infty}$ são constantes (com $\mathbf{f}_{\mathrm{ev}, \infty}>\mathbf{f}_{\mathrm{ev}, 0}$, para a curva crescente), e $\mathbf{f}_{\mathrm{sc}, 0}$ é o valor central da sigmóide referente à atividade eferente, descrita pela Equação 3-35.

\section{Parâmetros}

Os parâmetros para a atividade eferente simpática, extraídos de Ursino (1998), foram especificados para simular a queda porcentual na freqüência de impulsos no nervo renal canino em resposta a estimulação elétrica do nervo carotídeo, conforme medido por Wang, Brändle e Zucker ${ }^{23}$ (1993 apud URSINO, 1998). Devido à capacidade do nervo vago de transmitir tanto sinais aferentes quanto eferentes, os parâmetros relacionados à atividade eferente parassimpática tiveram que ser determinados de forma indireta, de modo a reproduzir as variações da freqüência cardíaca medidas em humanos em resposta a certas manobras, conforme reportado por Eckberg ${ }^{24}$ (1980) e Eckberg e Sleight ${ }^{25}$ (1992) apud Ursino, 1998.

Tabela 3-4 Parâmetros relacionados à descrição dos impulsos eferentes.

\begin{tabular}{|c|c|c|c|}
\hline \multicolumn{2}{|c|}{ Vias Eferentes Simpáticas } & \multicolumn{2}{|c|}{ Vias Eferentes Parassimpáticas } \\
\hline $\mathbf{f}_{\mathrm{es}, \infty}=2,10$ & impulsos/s & $\mathbf{f}_{\mathbf{e v}, 0}=3,2$ & impulsos/s \\
\hline $\mathbf{f}_{\mathrm{es}, 0}=16,11$ & impulsos/s & $\mathbf{f}_{\mathrm{ev}, \infty}=6,3$ & impulsos/s \\
\hline $\mathbf{f}_{\mathbf{e s}, \min }=2,66$ & impulsos/s & $\mathbf{f}_{\mathbf{s c}, 0}=25$ & impulsos/s \\
\hline $\mathbf{k}_{\text {es }}=0,0675$ & $\mathrm{~s}$ & $\mathbf{k}_{\mathbf{e v}}=7,06$ & s \\
\hline
\end{tabular}

Os subscritos 0 indicam ausência de inervação, ou seja, quando as atividades simpáticas ou parassimpáticas são nulas.

\footnotetext{
${ }^{23}$ WANG, W.; BRÄNDLE, M.; ZUCKER, I. H. Influence of vagotomy on the baroreflex sensitivity in anesthetized dogs with experimental heart failure. American Journal of Physiology: Heart and Circulatory Physiology, v. 265, p. H1310H1317, 1993.

${ }^{24}$ ECKBERG, D. L. Nonlinearities of the human carotid baroreceptor-cardiac reflex. Circulation Research, v.47, p. 208-216, 1980.

${ }^{25}$ ECKBERG, D. L.; SLEIGHT, P. Human baroreflexes in health and disease. Oxford: Oxford University Press, 1992.
} 


\subsubsection{Atuadores}

A relação qualitativa dos atuadores com as atividades simpáticas e parassimpáticas já foi descrita no item anterior. No modelo de Ursino, foram considerados como atuadores: a contratilidade do músculo cardíaco, a resistência periférica, o volume venoso não distendido e a freqüência cardíaca (na verdade, o período cardíaco). Todos estes quatro atuadores respondem a estímulos simpáticos, mas apenas o último responde à estimulação parassimpática.

No caso da estimulação simpática, foi descrita uma resposta genérica válida para todos os atuadores, e retratada por meio de um atraso, uma função estática logarítmica monotônica, e uma dinâmica linear de primeira ordem com constante de tempo real. A inclusão do atraso é justificada por diversos trabalhos (KATONA et al., 1970; DONALD e EDIS ${ }^{26}, 1971$; ROTHE ${ }^{27}, 1983$; SAGAWA ${ }^{28}, 1983$ apud URSINO, 1998) e desempenha importante papel na margem de estabilidade geral do sistema. O uso de relações logarítmicas é justificado com a resposta estática dos atuadores, que exibe uma inclinação elevada nas freqüências simpáticas baixas e um achatamento progressivo nas freqüências mais altas (KARIM e HAINSWORTH ${ }^{29}$, 1976; LEVY e ZIESKE ${ }^{30}, 1969$ apud URSINO, 1998). Apesar de a descrição ser a mesma, a função estática é monotonicamente crescente para a resistência sistêmica periférica e para a elastância no fim da sístole, mas monotonicamente decrescente para o período cardíaco e para os volumes venosos não distendidos. A dinâmica de primeira ordem serve para simular o tempo requerido para que o atuador progressivamente complete sua ação.

Assim, é possível escrever as equações gerais para os atuadores. Para evitar a repetição das equações, que são idênticas, será utilizada uma variável genérica, $\theta$, que denota o parâmetro contro-

${ }^{26}$ DONALD, D. E.; EDIS, A. J. Comparison of aortic and carotid baroreflexes in the dog. Journal of Physiology (London), v. 215, p. 521-538, 1971.

${ }^{27}$ ROTHE, C. F. Venous system: physiology of the capacitance vessels. In: Handbook of physiology. The cardiovascular system: Peripheral circulation and organ blood flow. Bethesda: Am. Physiol. Soc., 1983, sect. 2, vol. III, pt. 1, chapt. 13, p. 397-452.

${ }^{28}$ SAGAWA, K. Baroreflex control of systemic arterial pressure and vascular bed. In: Handbook of physiology. The cardiovascular system: Peripheral circulation and organ blood flow. Bethesda: Am. Physiol. Soc., 1983, sect. 2, vol. III, pt. 2, chapt. 14, p. 453-496.

${ }^{29}$ KARIM, F.; HAINSWORTH, R. Responses of abdominal vascular capacitance to stimulation of splanchnic nerves. American Journal of Physiology, v. 231, 434-440, 1976.

${ }^{30}$ LEVY, M. N.; ZIESKE, H. Autonomic control of cardiac pacemaker activity and atrioventricular transmission. Journal of Applied Physiology, v. 27, p. 465-470, 1969. 
lado, podendo ser substituída pelo próprio. Os parâmetros associados a $\theta$ são: $\mathbf{E}_{\max , \mathbf{V E}}, \mathbf{E}_{\max , \mathbf{V D}}$ ， $\mathbf{R}_{\mathbf{s p}}, \mathbf{R}_{\mathbf{e p}}, \mathbf{v}_{\mathbf{u}, \mathbf{s v}}$ e $\mathbf{V}_{\mathbf{u}, \mathbf{e v}}$. Sendo $\sigma_{\theta}$ a saída da característica estática, temos

$$
\begin{aligned}
& \sigma_{\theta}(\mathbf{t})= \begin{cases}\mathbf{G}_{\theta} \ln \left[\mathbf{f}_{\text {es }}\left(\mathbf{t}-\mathbf{D}_{\theta}\right)-\mathbf{f}_{\text {es,min }}+1\right] & \text { se } \mathbf{f}_{\text {es }} \geq \mathbf{f}_{\text {es, min }} \\
0 & \text { se } \mathbf{f}_{\text {es }}<\mathbf{f}_{\text {es, min }}\end{cases} \\
& \tau_{\theta} \frac{\mathbf{d} \Delta \theta}{\mathbf{d t}}(\mathbf{t})=-\Delta \theta(\mathbf{t})+\sigma_{\theta}(\mathbf{t}) \\
& \theta(\mathbf{t})=\Delta \theta(\mathbf{t})+\theta_{0}
\end{aligned}
$$

onde $\tau_{\theta}$ e $\mathbf{D}_{\theta}$ são respectivamente a constante de tempo e 0 atraso do parâmetro controlado, $\mathbf{f}_{\mathrm{es} \text {, min }}$ é a estimulação simpática mínima, e $\Delta \theta$ é a variação do parâmetro causada pela estimulação simpática. $\mathbf{G}_{\theta}$ é um fator de ganho constante, positivo para a contratilidade e para as resistências periféricas, e negativo para os volumes não distendidos.

Segundo Ursino, o período cardíaco está sujeito aos controles simpático e parassimpático, em ambos os casos através das mesmas características descritas para os demais atuadores - um atraso, uma função estática monotônica, e uma dinâmica linear de primeira ordem. No entanto, de acordo com Parker et al. ${ }^{31}$ (1984 apud URSINO, 1998) e Katona et al. (1970), foi assumido que a resposta vaga é linear, com o período cardíaco diretamente proporcional à estimulação parassimpática. O período cardíaco final é determinado então pela soma das variações induzidas pelos estímulos eferentes (positivas para a estimulação parassimpática e negativas para a simpática) com um nível constante que representa o período na ausência de inervação.

Assim, as variações no período cardíaco introduzidas pela estimulação simpática, $\Delta \mathbf{T}_{\mathbf{s}}$, podem ser obtidas por equações análogas às Equações 3-38 e 3-39, representando uma característica logarítmica mononicamente decrescente,

$$
\begin{aligned}
& \sigma_{\mathbf{T}, \mathbf{s}}(\mathbf{t})= \begin{cases}\mathbf{G}_{\mathbf{T}, \mathbf{s}} \ln \left[\mathbf{f}_{\mathrm{es}}\left(\mathbf{t}-\mathbf{D}_{\mathbf{T}, \mathbf{s}}\right)-\mathbf{f}_{\mathbf{e s}, \text { min }}+1\right] & \text { se } \mathbf{f}_{\mathbf{e s}} \geq \mathbf{f}_{\mathbf{e s}, \text { min }} \\
0 & \text { se } \mathbf{f}_{\mathbf{e s}}<\mathbf{f}_{\mathbf{e s}, \text { min }}\end{cases} \\
& \tau_{\mathbf{T}, \mathbf{s}} \frac{\mathbf{d} \Delta \mathbf{T}_{\mathbf{s}}}{\mathbf{d t}}(\mathbf{t})=-\Delta \mathbf{T}_{\mathbf{s}}(\mathbf{t})+\sigma_{\mathbf{T}, \mathbf{s}}(\mathbf{t})
\end{aligned}
$$

\footnotetext{
${ }^{31}$ PARKER, P.; CELLER, B. G.; POTTER, E. K.; McCLOSKEY, D. I. Vagal stimulation and cardiac slowing. Journal of the Autonomic Nervous System, v. 11, p. 226-231, 1984.
} 
onde $\tau_{\mathbf{T}, \mathbf{s}}$ e $\mathbf{D}_{\mathbf{T , S}}$ são respectivamente a constante de tempo e o atraso da componente simpática do período cardíaco, $\mathbf{f}_{\mathbf{e s} \text {,min }}$ é a estimulação simpática mínima, $\Delta \mathbf{T}_{\mathbf{s}}$ é a variação causada pela estimulação simpática, e $\mathbf{G}_{\mathbf{T}, \mathbf{s}}$ é um fator de ganho constante negativo.

A variação do período cardíaco em resposta aos estímulos vagos é então descrita através de uma relação linear, ou seja,

$$
\begin{aligned}
& \sigma_{\mathbf{T}, \mathbf{v}}(\mathbf{t})=\mathbf{G}_{\mathbf{T}, \mathbf{v}} \mathbf{f}_{\mathrm{ev}}\left(\mathbf{t}-\mathbf{D}_{\mathbf{T}, \mathbf{v}}\right) \\
& \tau_{\mathbf{T}, \mathbf{v}} \frac{\mathbf{d} \Delta \mathbf{T}_{\mathbf{v}}}{\mathbf{d t}}(\mathbf{t})=-\Delta \mathbf{T}_{\mathbf{v}}(\mathbf{t})+\sigma_{\mathbf{T}, \mathbf{v}}(\mathbf{t})
\end{aligned}
$$

onde $\tau_{\mathbf{T}, \mathbf{V}}, \mathbf{D}_{\mathbf{T}, \mathbf{v}}$ e $\Delta \mathbf{T}_{\mathbf{V}}$ são os equivalentes parassimpáticos dos parâmetros descritos para a resposta simpática acima. $\mathbf{G}_{\mathbf{T}, \mathbf{v}}$ é um fator de ganho constante positivo, pois o período cardíaco cresce com o aumento da freqüência de impulsos eferentes no nervo vago.

Finalmente, definindo $\mathbf{T}_{0}$ como o período na ausência de inervação cardíaca, obtém-se o período cardíaco total $\mathbf{T}$, através da soma das parcelas anteriores:

$$
\mathbf{T}=\Delta \mathbf{T}_{\mathbf{s}}+\Delta \mathbf{T}_{\mathbf{v}}+\mathbf{T}_{0}
$$

\section{Parâmetros}

Os valores associados à contratilidade dos ventrículos foram baseados em dados relatados por Suga, Sagawa e Kostiuk ${ }^{32}$ (1976), Sheriff et al. ${ }^{33}$ (1989) e Sagawa et al. ${ }^{34}$ (1988) apud Ursino (1998), que sugerem que a inclinação da função pressão-volume no final da sístole varia cerca de $30 \%$ de seu valor normal quando a pressão no seio carotídeo é reduzida de 200 para $50 \mathrm{mmHg}$. A mesma porcentagem foi assumida para o ventrículo direito. $\mathrm{O}$ atraso e a constante tempo foram ajustados para re-

\footnotetext{
32 SUGA, H.; SAGAWA, K.; KOSTIUK, D. P. Controls of ventricular contractility assessed by pressure-volume ratio $E_{\max }$. Cardiovascular Research, v. 10, p. 582-592, 1976.

${ }^{33}$ SHERIFF, D. D.; KASS, D.; MacANESPIE-TUNIN, C.; SAGAWA, K. Influence of carotid sinus baroreceptor reflex on left ventricular contractility in anesthetized dogs (Abstract). Circulation, v.80, suppl. II: II-553, 1989.

${ }^{34}$ SAGAWA, K. W.; MAUGHAN, H.; SUGA, H.; SUNAGAWA, K. Cardiac contraction and the pressure-volume relationship. New York: Oxford University Press, 1988.
} 
produzir a função de transferência em malha aberta entre a pressão carotídea e a elastância sistólica final, estimada por Kubota et al..$^{35}$ (1992 apud URSINO, 1998).

Para os ganhos das relações logarítmicas das resistências, foram utilizados os trabalhos de Shoukas e Brunner ${ }^{36}$ (1980 apud URSINO, 1998) e Potts, Hatanaka e Shoukas (1996), que indicam aumentos na resistência sistêmica total de cerca de $200 \%$ para quedas na pressão carotídea de 200 para $50 \mathrm{mmHg}$. A separação entre as circulações esplâncnica e extra-esplâncnica foi feita com base em outros trabalhos (BRUNNER, SHOUKAS e MacANESPIE ${ }^{37}$, 1981 e COX e BAGSHAW ${ }^{38}, 1975$ apud URSINO, 1998), que sugerem que a primeira é bem mais fraca que a segunda. As constantes de tempo foram obtidas de Ursino, Antonucci e Belardinelli (1994) e os atrasos foram retirados de Donald e Edis $^{39}$ (1971 apud URSINO, 1998).

Os ganhos das relações logarítmicas dos volumes não distendidos foram ajustados para que a variação total devido ao reflexo fosse de aproximadamente $15 \mathrm{ml} / \mathrm{kg}$, conforme sugerido por Shigemi, Brunner e Shoukas $^{40}$ (1994), Shoukas e Brunner ${ }^{41}$ (1980) e Shoukas e Sagawa ${ }^{42}$ (1973) apud Ursino (1998). As constantes de tempo e os atrasos para os volumes não distendidos são maiores que aqueles assumidos para as resistências, conforme sugerido por Rothe ${ }^{43}(1983)$ e Shoukas e Sagawa ${ }^{44}$ (1973) apud Ursino (1998).

${ }^{35}$ KUBOTA, T.; ALEXANDER, J.; ITAYA, R.; TODAKA, K.; SUGIMACHI, M.; SUNAGAWA, K.; NOSE, Y.; TAKESHITA, A. Dynamic effects of carotid sinus baroreflex on ventriculoarterial coupling studied in anesthetized dogs. Circulation Research, v. 70, p. 1044-1053, 1992.

${ }^{36}$ SHOUKAS, A. A.; BRUNNER, M. Epinephrine and the carotid sinus baroreceptor reflex: Influence on capacitive and resistive properties of the total systemic vascular bed of the dog. Circulation Research, v. 47, p. 249-257, 1980.

${ }^{37}$ BRUNNER, M. J.; SHOUKAS, A. A.; MacANESPIE, C. L. The effect of the carotid sinus baroreceptor reflex on blood flow and volume redistribution in the total systemic vascular bed. Circulation Research, v. 48, 274-285, 1981.

${ }^{38}$ COX, R. H.; BAGSHAW, R. J. Baroreceptor reflex control of arterial hemodynamics in the dog. Circulation Research, v. 37, p. $772-786,1975$.

${ }^{39}$ DONALD, D. E.; EDIS, A. J. Comparison of aortic and carotid baroreflexes in the dog. Journal of Physiology (London), v. 215, p. 521-538, 1971.

${ }^{40}$ SHIGEMI, K.; BRUNNER, M. J.; SHOUKAS, A. A. a- e $\beta$-adrenergic mechanisms in the control of vascular capacitance by the carotid sinus baroreflex system. American Journal of Physiology: Heart and Circulatory Physiology, v. 267, p. H201-H210, 1994.

${ }^{41}$ SHOUKAS, A. A.; BRUNNER, M. Epinephrine and the carotid sinus baroreceptor reflex: Influence on capacitive and resistive properties of the total systemic vascular bed of the dog. Circulation Research, v. 47, p. 249-257, 1980.

${ }^{42}$ SHOUKAS, A. A.; SAGAWA, K. Control of total systemic vascular capacity by the carotid sinus baroreceptor reflex. Circulation Research, v. 33, p. 22-32, 1973.

${ }^{43}$ ROTHE, C. F. Venous system: physiology of the capacitance vessels. In: Handbook of physiology. The cardiovascular system: Peripheral circulation and organ blood flow. Bethesda: Am. Physiol. Soc., 1983, sect. 2, vol. III, pt. 1, chapt. 13, p. 397-452.

${ }^{44}$ SHOUKAS, A. A.; SAGAWA, K. op. cit. 
Finalmente, os valores para as características estáticas e dinâmicas do período cardíaco foram obtidos dos dados experimentais apresentados por Levy e Zieske ${ }^{45}$ (1969 apud URSINO, 1998). Os valores das constantes de tempo e dos atrasos foram especificados considerando que o controle vago é extremamente rápido, medido em décimos de segundo, enquanto o controle simpático atua em alguns segundos (KATONA et al., 1970). Os parâmetros são resumidos na Tabela 3-5 abaixo.

Tabela 3-5 Parâmetros referentes aos atuadores.

\begin{tabular}{|c|c|c|c|c|c|c|c|c|c|}
\hline \multicolumn{3}{|c|}{ Ganhos } & \multicolumn{3}{|c|}{$\begin{array}{l}\text { Constantes de } \\
\text { Tempo }\end{array}$} & \multicolumn{2}{|l|}{ Atrasos } & \multicolumn{2}{|c|}{$\begin{array}{l}\text { Parâmetros na Ausência de } \\
\text { Inervação }\end{array}$} \\
\hline $\mathbf{G}_{\mathbf{E}_{\max , \mathbf{V E}}}$ & $=0,475$ & $\mathrm{mmHg} / \mathrm{ml} \cdot \mathrm{v}$ & $\tau_{\mathbf{E}_{\max , \mathbf{V E}}}$ & $=8$ & $\mathrm{~s}$ & $\mathbf{D}_{\mathbf{E}_{\max , \mathbf{V E}}}=2$ & $\mathrm{~s}$ & $\mathbf{E}_{\max \mathbf{V E}, 0}=2,392$ & $\mathrm{mmHg} / \mathrm{ml}$ \\
\hline $\mathbf{G}_{\mathbf{E}_{\max , \mathbf{V D}}}$ & $=0,282$ & $\mathrm{mmHg} / \mathrm{ml} \cdot \mathrm{v}$ & $\tau_{\mathbf{E}_{\max , \mathbf{V D}}}$ & $=8$ & $\mathrm{~s}$ & $\mathbf{D}_{\mathbf{E}_{\max , \mathbf{V D}}}=2$ & $\mathrm{~s}$ & $\mathbf{E}_{\max \mathbf{V D}, 0}=1,412$ & $\mathrm{mmHg} / \mathrm{ml}$ \\
\hline $\mathbf{G}_{\mathbf{R}, \mathbf{s p}}$ & $=0,695$ & $\mathrm{mmHg} \cdot \mathrm{s} / \mathrm{ml} \cdot \mathrm{v}$ & $\tau_{\mathbf{R}, \mathbf{s p}}$ & $=6$ & $\mathrm{~s}$ & $\mathbf{D}_{\mathbf{R}, \mathbf{s p}}=2$ & $\mathrm{~s}$ & $\mathbf{R}_{\mathbf{s p}, 0}=2,49$ & $\mathrm{mmHg} \cdot \mathrm{s} / \mathrm{ml}$ \\
\hline $\mathbf{G}_{\mathbf{R}, \mathbf{e p}}$ & $=0,53$ & $\mathrm{mmHg} \cdot \mathrm{s} / \mathrm{ml} \cdot \mathrm{v}$ & $\tau_{\mathbf{R}, \mathbf{e p}}$ & $=6$ & $\mathrm{~s}$ & $\mathbf{D}_{\mathbf{R}, \mathbf{e p}}=2$ & $\mathrm{~s}$ & $\mathbf{R}_{\text {ep }, 0}=0,78$ & $\mathrm{mmHg} \cdot \mathrm{s} / \mathrm{ml}$ \\
\hline $\mathbf{G}_{\mathbf{v}_{\mathbf{u}, \mathbf{s v}}}$ & $=-265,4$ & $\mathrm{ml} / \mathrm{v}$ & $\tau^{\mathbf{v}_{\mathbf{u}, \mathbf{s v}}}$ & $=20$ & s & $\mathbf{D}_{\mathbf{v}_{\mathbf{u}, \mathbf{s v}}}=5$ & $\mathrm{~s}$ & $\mathbf{V}_{\mathbf{u}_{\mathbf{s v}, 0}}=1435,4$ & $\mathrm{ml}$ \\
\hline $\mathbf{G}_{\mathbf{v}_{\mathbf{u}, \mathbf{e v}}}$ & $=-132,5$ & $\mathrm{ml} / \mathrm{v}$ & $\tau_{\mathbf{v}_{\mathbf{u}, \mathbf{e v}}}$ & $=20$ & $\mathrm{~s}$ & $\mathbf{D}_{\mathbf{v}_{\mathbf{u}, \mathbf{e v}}}=5$ & $\mathrm{~s}$ & $\mathbf{V}_{\mathbf{u}_{\mathbf{e v}, 0}}=1537$ & $\mathrm{ml}$ \\
\hline $\mathbf{G}_{\mathbf{T}, \mathbf{s}}$ & $s=-0,13$ & impulsos & $\tau_{\mathbf{T}, \mathbf{s}}$ & $=2$ & $\mathrm{~s}$ & $\mathbf{D}_{\mathbf{T}, \mathbf{s}}=2$ & $\mathrm{~s}$ & $\mathbf{T}_{0}=0,58$ & $\mathrm{~s}$ \\
\hline $\mathbf{G}_{\mathbf{T}, \mathbf{v}}$ & $=0,09$ & impulsos & $\tau_{\mathbf{T , v}}$ & $=1,5$ & $\mathrm{~s}$ & $\mathbf{D}_{\mathrm{T}, \mathbf{v}}=\frac{0}{2}$ & $\mathrm{~s}$ & & \\
\hline
\end{tabular}

A efeito de simplificação, $v$ indica impulsos por segundo. Os subscritos 0 (na última coluna) indicam parâmetros na ausência de atividade nervosa.

\subsection{Modelagem dos Átrios Ativos}

O modelo descrito na seção anterior foi simulado em Matlab e Simulink, conforme será mostrado no próximo capítulo. Os resultados obtidos nas simulações com tal modelo apresentaram elevada proximidade com dados da literatura para quase todos os compartimentos, conforme será mostrado no Capítulo 4, com exceção dos átrios, cujas curvas (sobretudo de pressão) exibiram traçados com ausência de características qualitativas importantes (ondas $a$, $c$ e $v$ ) - ver Figura 3-13 abaixo. Estas curvas são originadas, respectivamente, pela contração dos átrios (antes da sístole ventricular e da abertura das válvulas mitral e tricúspide), pela onda de pressão retrógrada provocada pela contração dos ventrículos, e pelo acúmulo de sangue vindo do retorno venoso (antes da abertura das válvulas).

${ }^{45}$ LEVY, M. N.; ZIESKE, H. Autonomic control of cardiac pacemaker activity and atrioventricular transmission. Journal of Applied Physiology, v. 27, p. 465-470, 1969. 

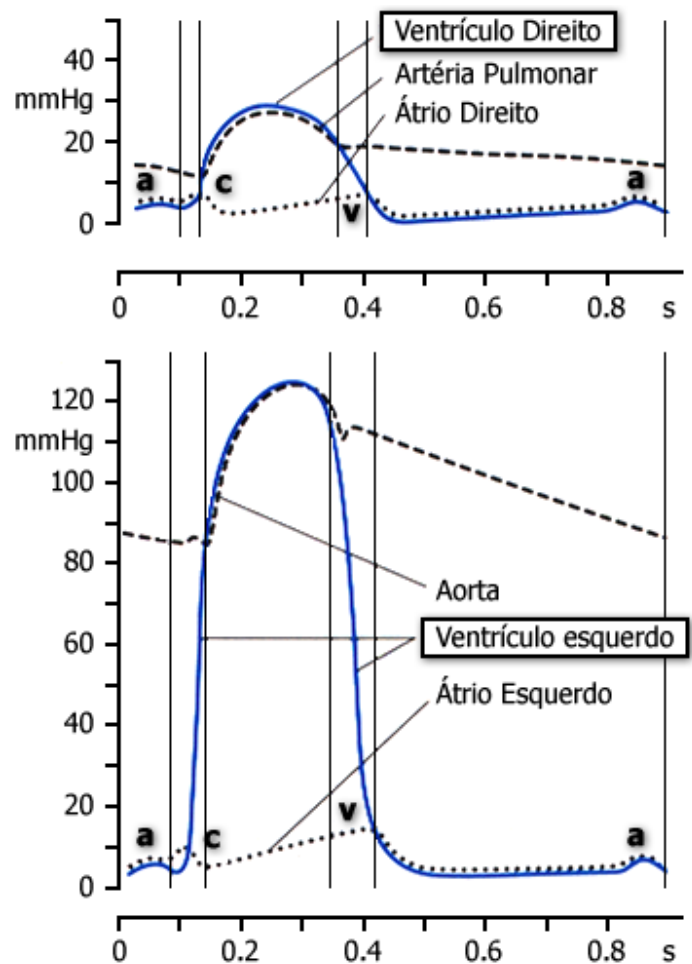

Figura 3-13 Ilustração das ondas $a$, ce v, para os lados direito e esquerdo do coração (adaptado de Antoni, 1996b).

A Figura 3-14 abaixo mostra um exemplo das simulações do modelo com átrios passivos mencionadas. Para a aplicação desejada, é de elevada importância que os átrios possuam um comportamento mais próximo da realidade, de modo a fornecer maior embasamento didático para os usuários do simulador. Por estes motivos, decidiu-se pela adição de átrios ativos ao modelo, utilizando como base o trabalho de Goodwin et al. (2004).

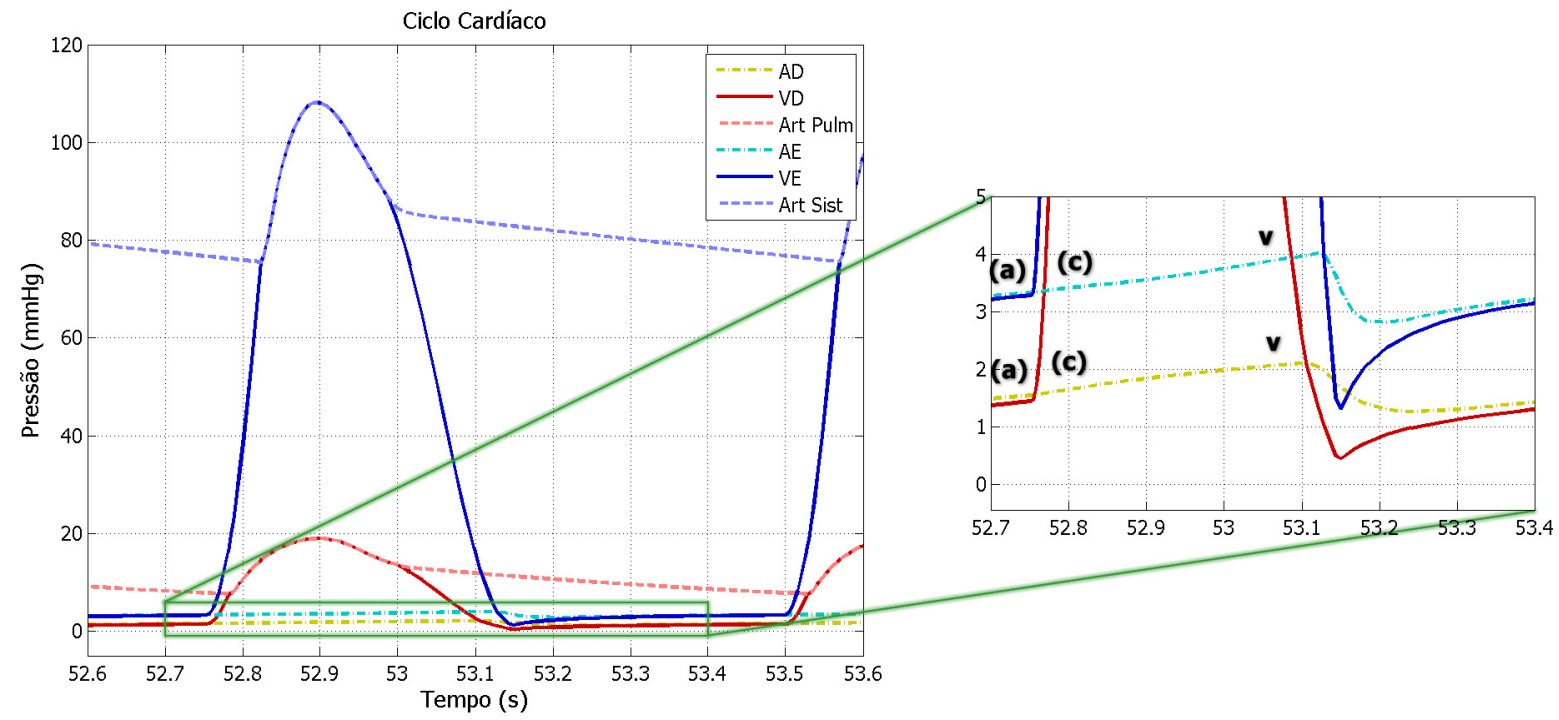

Figura 3-14 Simulação do ciclo cardíaco no modelo com átrios passivos, destacando as curvas de pressão atrial (direita e esquerda). Note que há apenas a formação da onda $v$, com ausência das ondas a e $c$ (as letras entre parênteses indicam as posições onde estas ondas deveriam aparecer). Além disso, os valores estão mais baixos que o esperado (entre 10 e 15 mmHg). 
Antes de ser mostrada a modelagem feita para os átrios, algumas considerações deverão ser feitas quanto aos aspectos que diferenciam seu comportamento daquele apresentado pelos ventrículos. Estes aspectos estão relacionados com a função de elasticidade e com a ativação do mecanismo de contração.

\subsubsection{Função Mecânica dos Átrios}

Apesar de inicialmente ter-se considerado a utilização nos átrios de uma simples adaptação do modelo dos ventrículos, percebeu-se algumas diferenças fundamentais entre o comportamento dinâmico dos mesmos. O trabalho de Goodwin et al. de 2004 apontava o uso de resistências constantes e elasticidades lineares tanto na sístole quanto na diástole atriais - diferentemente da relação exponencial considerada na diástole ventricular. Lembrando que a função da elasticidade ventricular fora determinada a partir da respectiva alça pressão-volume, passou-se à observação do mesmo tipo de curva referente aos átrios. Não foi encontrada uma grande quantidade de publicações tratando da função mecânica dos átrios, mas alguns trabalhos na área de cardiologia e anestesiologia (e não de modelagem matemática) evidenciam o uso da alça pressão-volume na estimação dos valores de elasticidade (a estimação de parâmetros será tratada mais adiante). A relação pressão-volume atrial é bastante diferente da ventricular, descrevendo uma dupla alça, na forma de um algarismo oito deitado, conforme Figura 3-15 abaixo (painéis da direita).

Antes de ser explicada a determinação das curvas de elasticidade atrial, passaremos à descrição da função mecânica dos átrios, começando pelo lado esquerdo.

\section{Alça Pressão-Volume do Átrio Esquerdo}

As figuras e a explanação a seguir referem-se ao átrio esquerdo, mas como será mostrado mais adiante, satisfazem também ao lado direito. A Figura 3-15 abaixo apresenta dois conjuntos de valores, identificados por (a) e (b). O painel (a), superior, apresenta curvas extraídas do trabalho de Pagel et al., de 2003, enquanto (b), o painel inferior, mostra valores obtidos de Matsuda et al., de 1983. Ambos foram adaptados para compatibilização dimensional. Seria suficiente mostrar apenas um dos grupos de dados, mas deseja-se enfatizar aqui a dificuldade de medição destas grandezas, o que 
acaba por gerar certa discrepância entre diferentes estudos. Devido ao difícil acesso, a maior parte dos trabalhos sobre o átrio esquerdo apresenta dados obtidos indiretamente, através de ecocardiografias com Doppler (PAGEL et al., 2003) ou angiocardiografias (HAWLEY, DODGE e GRAHAM, 1966), às vezes com o auxílio de cateteres inseridos através da artéria braquial (MATSUDA et al., 1983). Apesar de no átrio direito o uso de cateteres invasivos ser mais comum (como nas medições de débito cardíaco com cateteres de Swan-Ganz), ocorre a mesma variação entre diferentes publicações, conforme será mostrado mais adiante, devido à relativa imprecisão das medições. Observe, por exemplo, que apesar de ambos os resultados mostrados na Figura 3-15 abaixo corresponderem a pacientes em condições de normalidade, há algumas diferenças relevantes entre os dois, como a ordem de grandeza dos volumes e os picos de pressão ( $a$ e $v$ ), que aparecem com intensidades máximas trocadas.
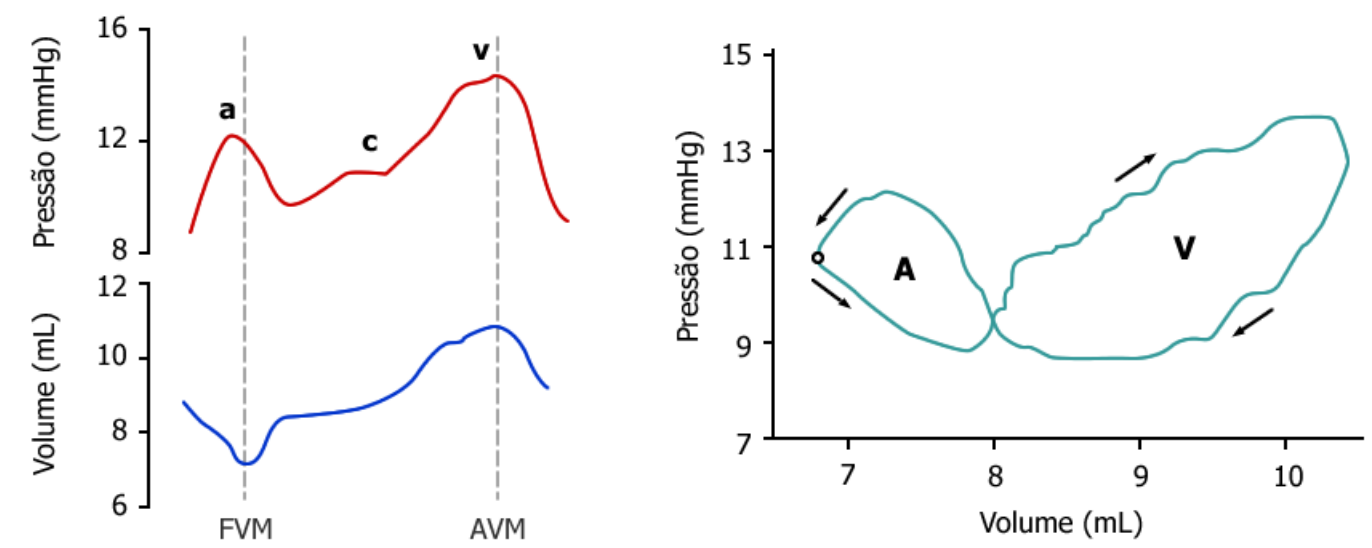

(a)
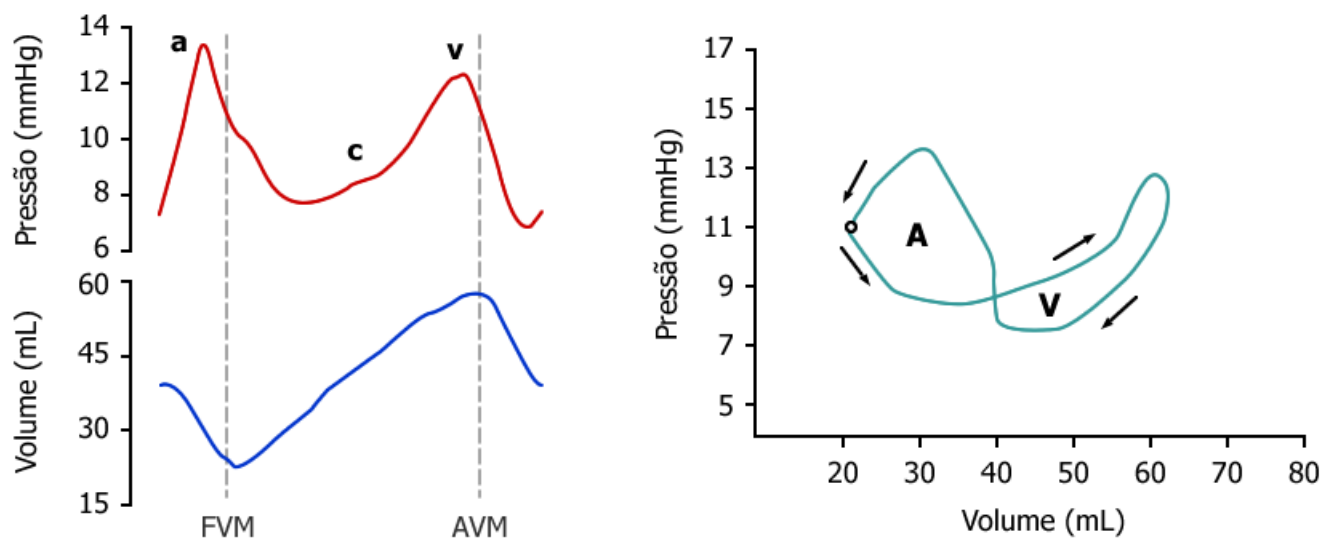

(b)

Figura 3-15 Curvas de pressão e volume no átrio esquerdo, conforme mostrado por diferentes trabalhos. Os traçados de pressão e volume e a alça em (a) foram adaptados de Pagel et al. (2003), que utilizaram ecocardiografias com Doppler para estimação dos valores. Os traçados em (b) foram adaptados de Matsuda et al. (1983), que utilizaram angiocardiografias auxiliadas por cateteres com micro-manômetros. FVM significa o fechamento da válvula mitral, representado nas alças pelo pequeno círculo preto à esquerda do traçado, e $A V M$ significa a abertura da mesma válvula. Nas alças pressão-volume, é representado um ciclo cardíaco, iniciado no fechamento da válvula mitral. As denominações das regiões dos gráficos são as encontradas na literatura $(a, c$ e $v$ nos traçados de pressão e $A$ e $V$ nas alças). 
Feitas estas ressalvas, pode-se prosseguir à descrição da função mecânica dos átrios. Conforme mencionado, apesar de ser utilizado o átrio esquerdo na explanação, o funcionamento é semeIhante para o lado direito. $\mathrm{O}$ início do ciclo é considerado no final da diástole, representada pelos cruzamentos nas alças da Figura 3-15 acima, pouco antes do fechamento da válvula mitral (ponto representado pelo pequeno círculo à esquerda das alças). O traçado inicia descrevendo a componente ativa da função atrial (região A), em sentido anti-horário, a partir do início da contração do átrio (após a onda $\mathrm{P}$ de despolarização dos mesmos no eletrocardiograma). O sangue é ejetado pelo aumento de pressão (onda a nos traçados respectivos), provocando a queda do volume. Após o pico de pressão e o início do relaxamento do átrio, ocorre o fechamento da válvula mitral (linha tracejada identificada por FVM), devido à contração do ventrículo. Isso provoca a transmissão para o átrio de uma onda de pressão retrógrada, devida ao abaulamento da válvula mitral, causando um aumento na pressão atrial (caracterizado pela onda $c$ dos traçados ${ }^{46}$ ). Desde o fechamento da válvula mitral o átrio já iniciara seu enchimento (com o sangue vindo das veias pulmonares), conforme observado nos traçados de volume, caracterizando também o segmento ascendente da componente passiva da função atrial (região $\mathbf{V}$ das alças, em sentido horário). Durante o final da contração ventricular isovolumétrica, da ejeção ventricular e da maior parte do relaxamento ventricular isovolúmico, ocorre um aumento gradativo da pressão e do volume atriais, resultando na onda $v$ dos traçados de pressão ${ }^{47}$. Com a abertura da válvula mitral, ocorre novo declínio na pressão, devido à queda de volume originada pela passagem (passiva) do sangue para o ventrículo. Esta diminuição de pressão e volume descreve o segmento descendente da alça $\mathbf{V}$, fechando o ciclo completo (PAGEL et al. 2003; MANIAR et al., 2003; MILLER et al., 1986; HAWLEY et al., 1966; GUYTON e HALL, 1997).

\section{Alça Pressão-Volume do Átrio Direito}

Conforme mencionado, a relação pressão-volume para o átrio direito é bastante semelhante à do lado esquerdo. Além dos traçados de pressão e volume serem semelhantes, a alça também se

\footnotetext{
${ }^{46} \mathrm{~A}$ onda $c$ pode ser mais pronunciada na presença de prolapso da válvula mitral, que provoca abaulamento excessivo das cúspides (PAGEL et al, 2003). Este caso não será considerado na modelagem proposta.

${ }^{47}$ A onda $v$ também pode ter sua intensidade acentuada, caso ocorra regurgitação valvular (PAGEL et al. 2003; MILLER et al., 1986; GUYTON e HALL, 1996). Novamente, este caso não será considerado na modelagem aqui desenvolvida.
} 
comporta da mesma forma, descrevendo a figura de um algarismo oito tombado, conforme Figura 3-16 abaixo. A descrição do ciclo cardíaco para o lado direito é a mesma apresentada acima, trocando-se apenas as estruturas anatômicas equivalentes - válvula tricúspide no lugar da válvula mitral, veias esplâncnicas e extra-esplâncnicas no lugar das veias pulmonares. As alças $\mathbf{A}$ e $\mathbf{V}$ continuam correspondendo às componentes ativa e passiva, respectivamente, da função atrial.
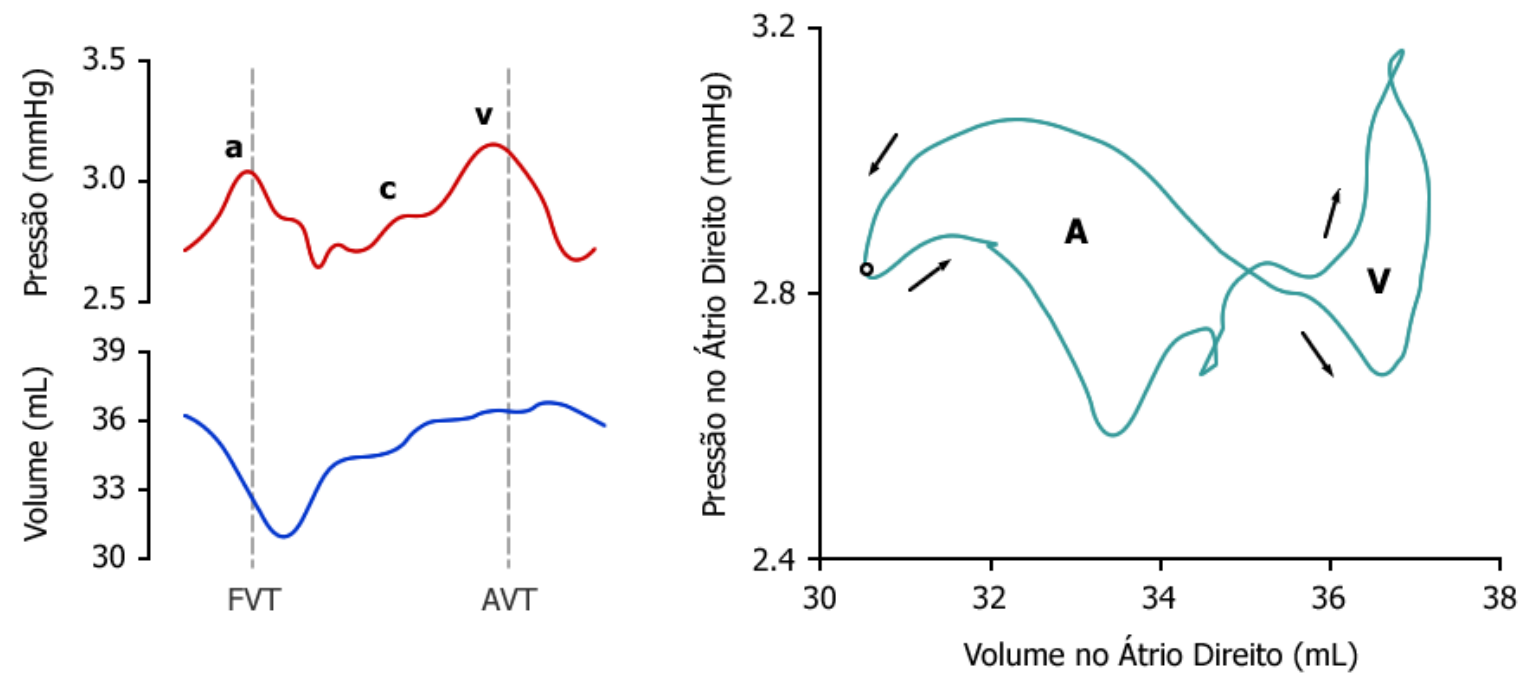

(a)
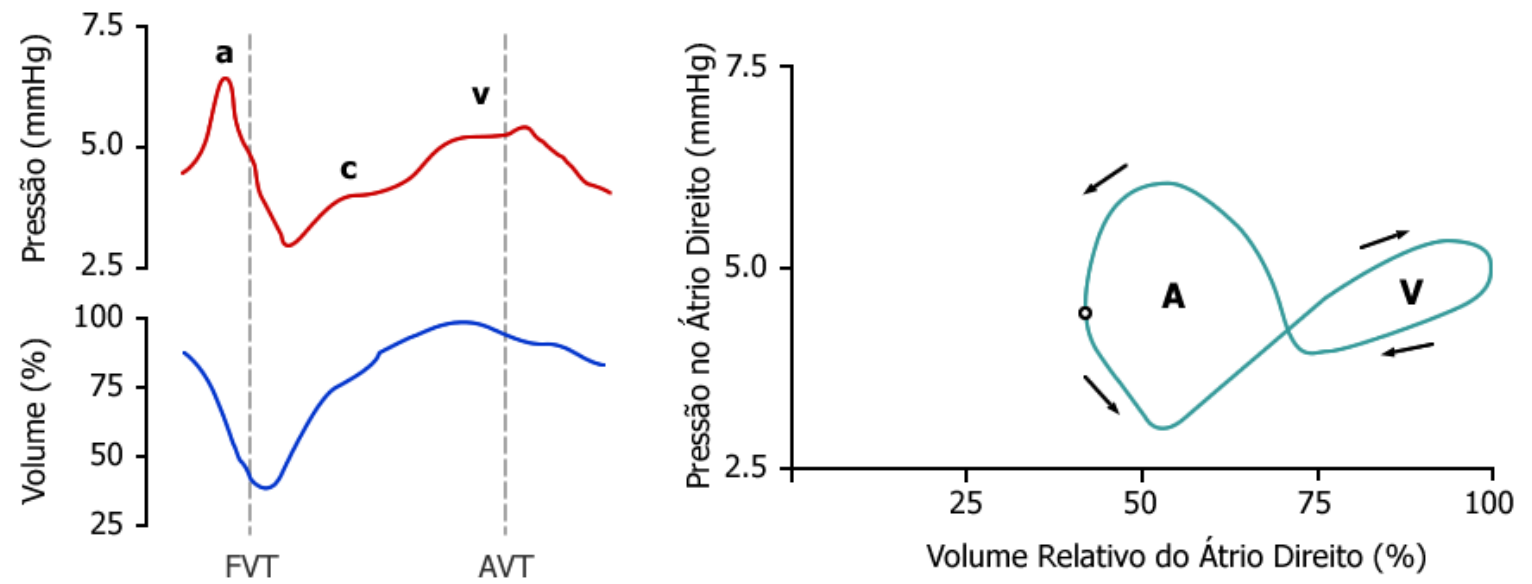

(b)

Figura 3-16 Curvas de pressão e volume no átrio direito, conforme mostrado por diferentes trabalhos. Os gráficos em (a) foram adaptados de Maniar et al. (2003), que utilizaram cateteres especificamente construídos para a estimação das pressões e volumes através da medição de condutância em oveIhas. Os traçados em (b) foram adaptados de Miller et al. (1986), que utilizaram cateteres de impedância para estimação dos volumes e micro-manômetros para medição de pressão em porcos. Note que este trabalho utiliza unidades relativas de volume, pois o método é incapaz de fornecer uma estimativa dos valores absolutos. FVT significa o fechamento da válvula tricúspide, representado nas alças pelo pequeno círculo preto à esquerda do traçado, e $A V T$ significa a abertura da mesma válvula. Nas alças pressão-volume, é representado um ciclo cardíaco, iniciado no fechamento da válvula tricúspide. 
Mais uma vez pode-se notar nas curvas, agora da Figura 3-16, que tanto a forma quanto os valores absolutos continuam variando bastante entre diferentes publicações. Esta figura mostra, utilizando a mesma compatibilização dimensional adotada na Figura 3-15, dados extraídos de Maniar et al. (2003) no painel superior (a), e dados obtidos de Miller et al. (1986) no painel (b), inferior. Isto reforça a colocação proposta anteriormente de inconsistência nos dados experimentais de diferentes fontes.

\section{Estimação das Funções de Elasticidade}

A estimação das elasticidades propriamente ditas é feita a partir da plotagem de uma seqüência de alças pressão-volume sobrepostas. A variação da pré-carga durante consecutivos ciclos cardíacos descreve, no caso dos átrios, duas retas, que caracterizam as elasticidades mínima (EMIN) e máxima (EMAX), conforme Figura 3-17 abaixo. Estas retas são formadas respectivamente pelo lugar geométrico da pressão diastólica final e da pressão sistólica final (PAGEL et al., 2003; MANIAR et al., 2003; GOODWIN et al., 2004).

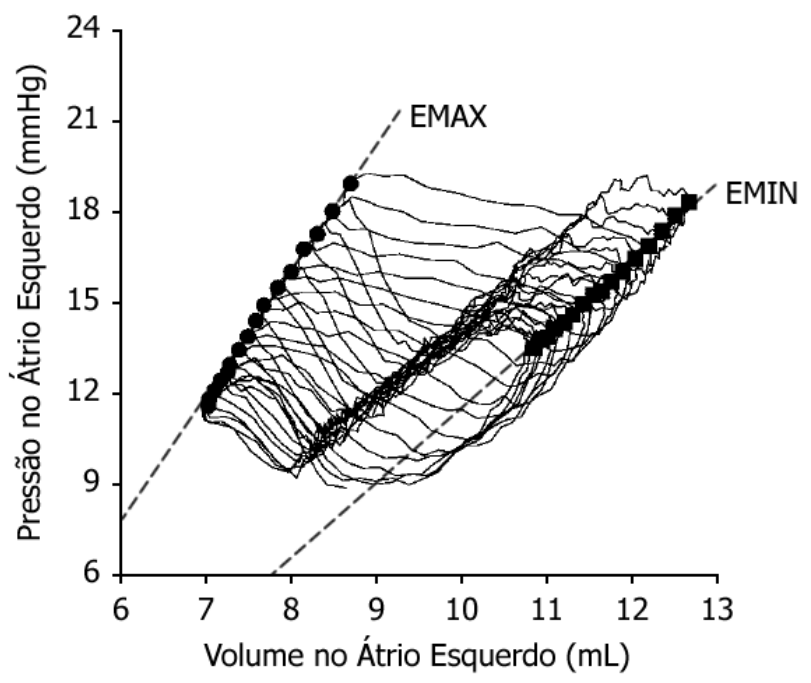

Figura 3-17 Determinação das elastâncias para o átrio esquerdo. EMAX é a elastância máxima, obtida pela inclinação da reta que liga os pontos correspondentes ao fim da sístole atrial. EMIN é a elastância mínima, determinada através da inclinação da reta obtida pela união dos pontos correspondentes ao final da diástole atrial (adaptado de Pagel et al., 2003).

As funções de elasticidade são determinadas a partir da inclinação destas retas (PAGEL et al. 2003; MANIAR et al., 2003), cujo cruzamento com o eixo das abscissas delimita o volume não distendido do respectivo átrio. No caso dos ventrículos, os trabalhos de modelagem consultados (URSINO, 1998; GOODWIN et al., 2004) indicavam a utilização do cruzamento da reta de elasticidade máxima, 
formada pelos pontos de pressão sistólica final, para a determinação do volume não distendido. Ao utilizarem uma reta também para a elasticidade mínima (diferentemente de Ursino, 1998), Goodwin et al. (2004) assumem que as duas retas se cruzam sobre o eixo horizontal, definindo aí o volume não distendido do ventrículo. Baseado no trabalho de Maniar et al. de 2003, relacionado à função mecânica do átrio direito, foi assumida uma característica semelhante à utilizada por Goodwin et al. (2004), ou seja, foram escolhidas elasticidades que fizessem com que o volume não distendido se localizasse efetivamente sobre o cruzamento das duas retas com o eixo dos volumes. A estimação dos parâmetros será descrita mais adiante.

A Figura 3-18 abaixo mostra curvas de elasticidade do átrio direito extraídas do referido trabaIho de Maniar et al. (2003). Neste estudo foram utilizadas ovelhas para a avaliação da função mecânica do átrio direito em dependência de variações de carga. Os dados correspondem a uma seqüência típica de alças pressão-volume para variadas pré-cargas em um animal em condições de normalidade. É interessante observar que neste trabalho a elasticidade máxima, correspondente aos pontos de fim da sístole, é denotada simplesmente por elasticidade do átrio, enquanto a elasticidade mínima, correspondente aos pontos de fim da diástole, é denotada por rigidez do átrio. Esta notação não será aqui adotada, no entanto, para manter-se a consistência com a modelagem dos ventrículos.

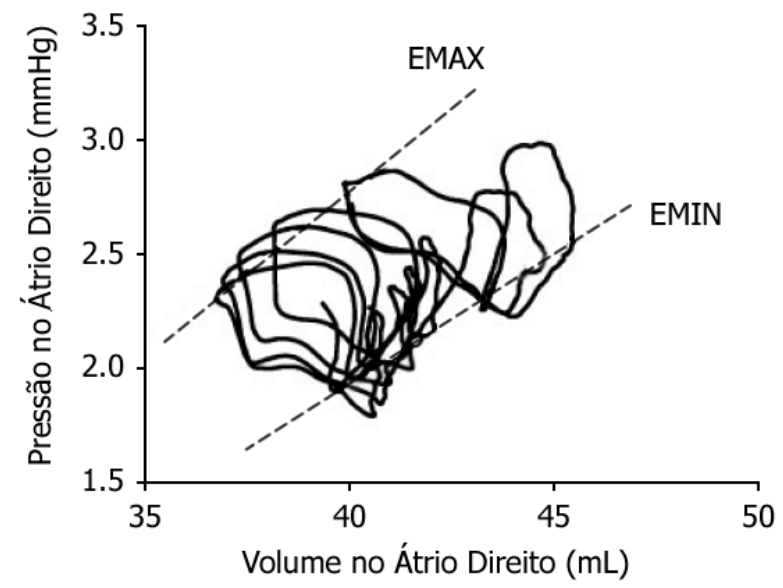

Figura 3-18 Determinação das elastâncias do átrio direito. (adaptado de Maniar et al., 2003).

Tanto as elasticidades quanto o volume não distendido podem variar devido a diversos fatores, como os mencionados prolapso das válvulas (mais comum na válvula mitral) e regurgitação valvular, ou ainda devido à administração de drogas (PAGEL et al. 2003; MANIAR et al., 2003; MILLER et al., 1986; HAWLEY et al., 1966). 


\subsubsection{Função de Ativação}

A modelagem dos átrios ativos requer ainda mais uma diferenciação em relação à dos ventrículos. Observando alguns resultados preliminares, foi possível perceber que a função de ativação dos átrios não pode ser a mesma utilizada nos ventrículos. A diferença mais evidente (e já contabilizada no modelo) está no momento em que se inicia a contração. Segundo Guyton e Hall (1997), a contração dos átrios ocorre 160 ms antes da contração dos ventrículos, devendo-se este atraso à propagação do estímulo elétrico do Nodo Sinoatrial (ou Nodo SA) ao Nodo Atrioventricular (ou Nodo AV). A Figura 3-19 mostra estes nodos, bem como os demais feixes por onde o estímulo é conduzido.

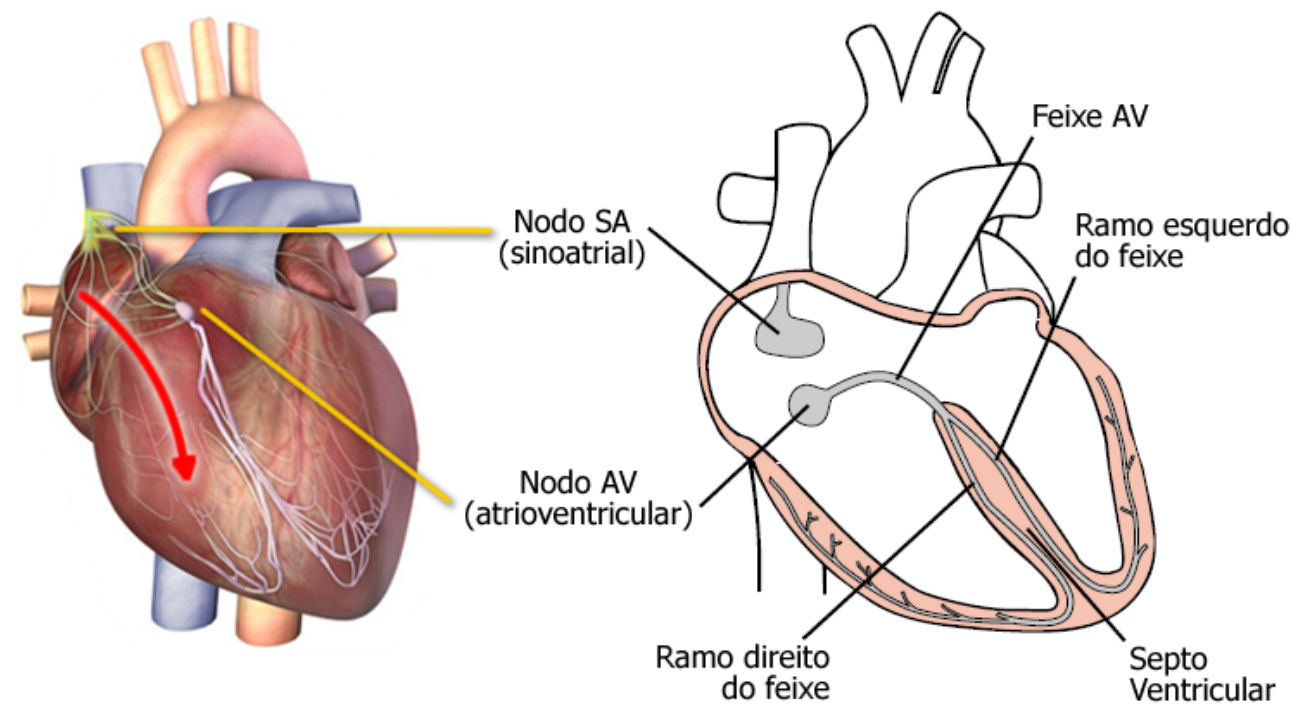

Figura 3-19 Propagação do estímulo elétrico pelo músculo cardíaco (a seta vermelha indica o sentido de condução). O impulso rítmico normal é gerado no Nodo SA, ou sinoatrial (ou ainda sinusa) e conduzido até o Nodo AV, ou Atrioventricular, sofrendo um retardo de 160 ms. 0 impulso continua pelo Feixe AV e é conduzido para os feixes esquerdo e direito das fibras de Purkinje, que distribuem o impulso por todas as partes dos ventrículos. (Adaptado de Rhoades e Tanner, 2003 e 3DScience.com, com base em Guyton e Hall, 1997).

Assim que o impulso rítmico normal é gerado no Nodo SA, inicia-se a contração dos átrios. Após o mencionado retardo no Nodo AV, o impulso continua pelo Feixe Atrioventricular (ou Feixe AV), que se divide nos dois ramos principais (esquerdo e direito) do sistema de fibras de Purkinje. Este sistema de fibras estende-se sobre os ventrículos, levando o impulso quase que simultaneamente ${ }^{48} \mathrm{a}$ todas as fibras musculares, iniciando-se então a contração dos ventrículos (GUYTON e HALL, 1997).

\footnotetext{
${ }^{48}$ A diferença de tempo máxima , correspondente às fibras mais distantes, é de aproximadamente 60 ms (GUYTON e HALL, 1997; FOX, 2006).
} 
Além do atraso de propagação, há um segundo fator diferenciador na função de ativação dos átrios. A duração de sua contração é menor que a duração da contração dos ventrículos, conforme mostra a Figura 3-20 abaixo. A figura, correspondente a medições feitas em coelhos, indica que os potenciais de ação dos átrios e dos ventrículos são mantidos por durações diferentes (ANTONI, 1996a). Desta forma, a duração das contrações também é diferente.

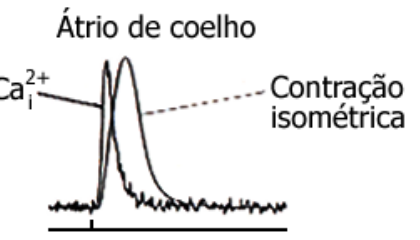

Ventrículo de coelho

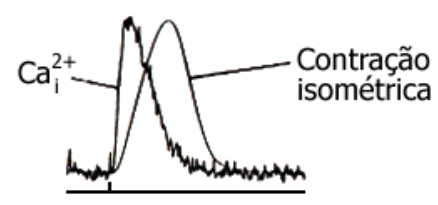

Figura 3-20 Diferenças na duração da contração dos átrios e dos ventrículos. Antoni (1996) mostra as durações dos potenciais de ação em um coelho. (Adaptado de Antoni, 1996a).

Segundo Fox (2006), para uma freqüência de 75 batimentos por minuto, a sístole e a diástole ventriculares duram respectivamente 0,3 e 0,5 segundos, enquanto a sístole e a diástole atriais duram respectivamente 0,1 e 0,7 segundos (a contração dura 0,1 segundo e o relaxamento dura 0,3 segundos). O diagrama da Figura 3-21 abaixo ilustra melhor a relação entre as durações dos eventos de contração e relaxamento das câmaras do coração.

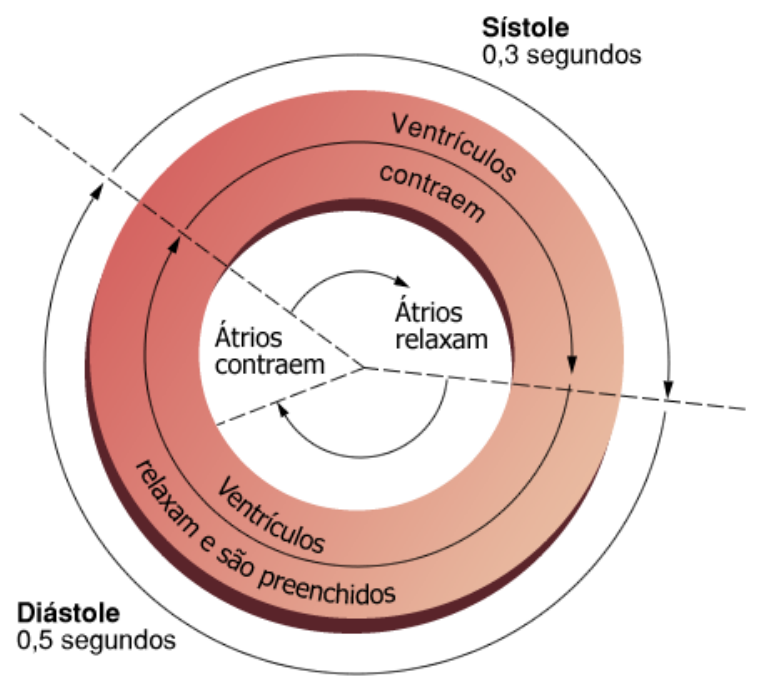

Figura 3-21 Temporização dos eventos do ciclo cardíaco. A sístole atrial é iniciada no final da diástole ventricular. A diástole atrial ocorre durante a sístole ventricular, permanecendo os átrios relaxados durante o restante do ciclo. As durações mostradas são relativas a uma freqüêencia cardíaca de 75 batimentos por minuto. (Adaptado de Fox, 2006). 
Desta forma, a função de ativação dos átrios precisou não apenas ser adiantada como também estreitada, reduzindo a duração de sua contração em relação à dos ventrículos. Foi selecionado um fator tal que a duração da contração atrial ficasse três vezes menor que a duração da contração ventricular, conforme a Figura 3-22 abaixo.

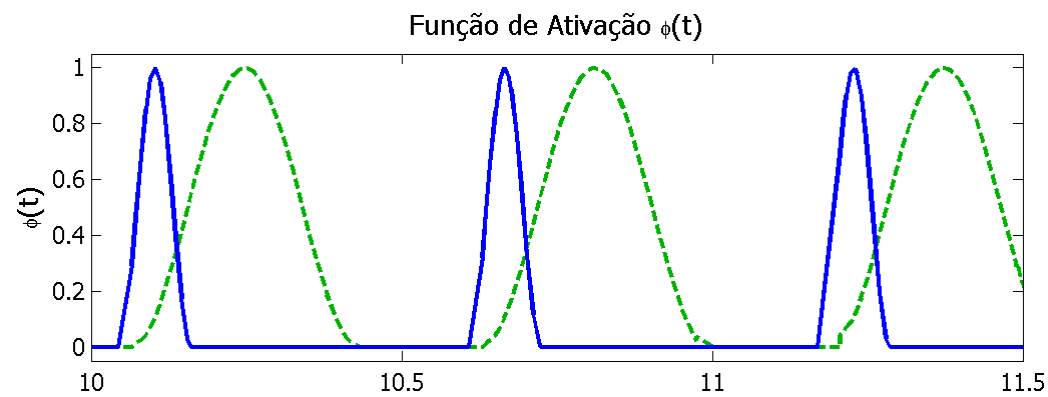

Figura 3-22 Função de ativação dos átrios (azul) e dos ventrículos (tracejado verde).

\subsubsection{Modelagem}

A modelagem dos átrios ativos foi feita então com base no referido modelo de Goodwin et al. de 2004 e nos trabalhos sobre a função mecânica atrial, sobretudo no apresentado por Maniar et al. de 2003. As resistências atriais foram consideradas constantes (GOODWIN et al., 2004), diferentemente das resistências variáveis utilizadas anteriormente na modelagem dos ventrículos. Conforme previamente descrito, as elastâncias também foram consideradas constantes, obedecendo a funções lineares tanto para a contração quanto para o relaxamento. Não foi incluída, portanto, atuação nervosa sobre os átrios (contrariamente ao caso dos ventrículos). A descrição do modelo será iniciada pelo lado esquerdo do coração.

\section{Lado Esquerdo}

Com base nas considerações feitas acima, foi assumido que a atividade contrátil dos átrios é caracterizada por um arranjo em série de uma resistência constante e uma elastância variável. A elastância varia durante o ciclo cardíaco em função da atividade contrátil do átrio, e contabiliza a relação isométrica entre pressão e volume.

Definindo $\dot{\mathbf{Q}}_{\mathbf{e s q}, \mathbf{e}}$ como sendo o fluxo de entrada no ventrículo esquerdo (e conseqüentemente o fluxo de saída do átrio esquerdo), e sendo $\dot{\mathbf{Q}}_{\mathbf{A E}}$ o fluxo de entrada no átrio esquerdo, vindo das 
veias pulmonares, pode-se escrever a equação da conservação de massa de modo a se obter o volume no átrio esquerdo $\mathbf{V}_{\mathbf{A E}}$ :

$$
\frac{\mathbf{d} \mathbf{V}_{\mathrm{AE}}}{\mathbf{d t}}=\dot{\mathbf{Q}}_{\mathrm{AE}}-\dot{\mathbf{Q}}_{\mathrm{esq}, \mathrm{e}}
$$

com $\dot{\mathbf{Q}}_{\mathbf{A E}}$ dado por

$$
\dot{\mathbf{Q}}_{\mathbf{A E}}=\frac{\mathbf{P}_{\mathrm{pv}}-\mathbf{P}_{\mathbf{A E}}}{\mathbf{R}_{\mathrm{pv}}}
$$

O fluxo de entrada no ventrículo esquerdo, $\dot{\mathbf{Q}}_{\mathbf{e s q}, \mathbf{e}}$, depende da abertura da válvula mitral, e pode ser descrito por

$$
\dot{\mathbf{Q}}_{\text {esq,e }}= \begin{cases}0 & \text { se } \mathbf{P}_{\mathrm{max}, \mathbf{A E}} \leq \mathbf{P}_{\mathbf{V E}} \\ \frac{\mathbf{P}_{\mathrm{max}, \mathbf{A E}}-\mathbf{P}_{\mathbf{V E}}}{\mathbf{R}_{\mathbf{A E}}} & \text { se } \mathbf{P}_{\mathrm{max}, \mathbf{A E}}>\mathbf{P}_{\mathbf{V E}}\end{cases}
$$

onde $\mathbf{P}_{\max , \mathbf{A E}}$ é a função de elasticidade do átrio esquerdo, e $\mathbf{R}_{\mathbf{A E}}$ é a respectiva resistência viscosa, considerada constante. Desta forma, a pressão instantânea no átrio esquerdo $\mathbf{P}_{\mathbf{A E}}$ resulta da diferença entre a pressão isométrica e as perdas resistivas, de modo a contabilizar os efeitos da contratilidade do músculo e da resistência à passagem do sangue. Assim,

$$
\mathbf{P}_{\mathbf{A E}}=\mathbf{P}_{\max , \mathbf{A E}}-\mathbf{R}_{\mathbf{A E}} \dot{\mathbf{Q}}_{\text {esq, } \mathbf{e}}
$$

A função pressão-volume dos átrios depende de duas componentes - uma para o enchimento e outra para o relaxamento. Segundo Maniar et al. (2003), a seguinte equação rege a elasticidade do átrio durante seu enchimento ${ }^{49}$ :

$$
\mathbf{P}_{\text {sis }, \mathbf{A E}}=\mathbf{E}_{\max , \mathbf{A E}}\left(\mathbf{V}_{\text {sis }, \mathbf{A E}}-\mathbf{V}_{\mathbf{u}, \mathbf{A E}}\right)
$$

sendo que o índice sis se refere aos valores no fim da sístole atrial. A elasticidade máxima, $\mathbf{E}_{\max , \mathbf{A E}}$, é a mesma descrita anteriormente como EMAX, na Seção 3.3.1. De forma semelhante, escreve-se a equação do relaxamento do átrio, com o subscrito dia referindo-se aos valores no fim da diástole:

$$
\mathbf{P}_{\mathrm{dia}, \mathrm{AE}}=\mathbf{E}_{\min , \mathbf{A E}}\left(\mathbf{V}_{\mathrm{dia}, \mathrm{AE}}-\mathbf{V}_{\mathbf{u}, \mathrm{AE}}\right)
$$

\footnotetext{
${ }^{49}$ No trabalho de Maniar et al. (2003), as Equações 3-50 e 3-51 referem-se originalmente ao átrio direito, mas foram aqui adaptadas também ao átrio esquerdo.
} 
onde $\mathbf{E}_{\min , \mathbf{A E}}$ é a elasticidade mínima, conforme descrita anteriormente na Seção 3.3.1 com o nome de EMIN. Note que o volume não distendido $\mathbf{V}_{\mathbf{u}, \mathbf{A E}}$ é o mesmo nas duas equações, concordando com a proposição anterior de que as duas retas se cruzariam sobre o eixo dos volumes na alça pressãovolume.

Finalmente, pode-se escrever a função pressão-volume completa, utilizando as Equações 3-50 e 3-51:

$$
\begin{array}{r}
\mathbf{P}_{\max , \mathbf{A E}}(\mathbf{t})=\varphi_{\mathbf{a}}(\mathbf{t}) \mathbf{E}_{\max , \mathbf{A E}}\left(\mathbf{V}_{\mathbf{A E}}-\mathbf{V}_{\mathbf{u}, \mathbf{A E}}\right)+\left[1-\varphi_{\mathbf{a}}(\mathbf{t})\right] \mathbf{E}_{\min , \mathbf{A E}}\left(\mathbf{V}_{\mathbf{A E}}-\mathbf{V}_{\mathbf{u}, \mathbf{A E}}\right) \\
0 \leq \varphi_{\mathbf{a}}(\mathbf{t}) \leq 1
\end{array}
$$

Note que $\mathbf{V}_{\mathbf{A E}}$ é o mesmo nas duas parcelas, pois as componentes sistólica e diastólica são agora reguladas pela função de ativação $\varphi_{\mathbf{a}}(\mathbf{t})$, tornando os índices sis e dia desnecessários. A função de ativação pulsante dos átrios $\varphi_{\mathbf{a}}(\mathbf{t})$, que governa a transição entre sístole e diástole, utiliza o mesmo período $\mathbf{T}$ considerado na função de ativação dos ventrículos (agora rebatizada como $\varphi_{\mathbf{v}}(\mathbf{t})$ ), e igual ao período cardíaco (que corresponde ao inverso da freqüência cardíaca). Da mesma forma que nos ventrículos, foi utilizada uma senóide quadrada para a descrição de $\varphi_{\mathbf{a}}(\mathbf{t})$, possuindo valor 1 na máxima contração e valor 0 no máximo relaxamento,

$$
\varphi_{\mathbf{a}}(\mathbf{t})= \begin{cases}\operatorname{sen}^{2}\left[\frac{\pi \mathbf{T}(\mathbf{t})}{\mathbf{T}_{\text {sis, } \mathbf{a}}(\mathbf{t})} \cdot \mathbf{u}(\mathbf{t})\right] & 0 \leq \mathbf{u}(\mathbf{t}) \leq \frac{\mathbf{T}_{\text {sis, } \mathbf{a}}}{\mathbf{T}} \\ 0 & \frac{\mathbf{T}_{\text {sis, } \mathbf{a}}}{\mathbf{T}} \leq \mathbf{u}(\mathbf{t}) \leq 1\end{cases}
$$

A diferença aqui está na duração da sístole atrial $\mathbf{T}_{\mathbf{a}, \text { sis }}$, menor que a duração da sístole ventricular, rebatizada como $\mathbf{T}_{\mathbf{v}, \text { sis }}{ }^{50}$. O adimensional $\mathbf{u}$ é o mesmo utilizado nos ventrículos, variando entre 1 e 0, representando a fração do ciclo cardíaco. A equação é transcrita abaixo.

$$
\mathbf{u}(\mathbf{t})=\operatorname{rem}\left[\int_{\mathbf{t}_{0}}^{\mathbf{t}} \frac{1}{\mathbf{T}(\tau)} \mathbf{d} \tau+\mathbf{u}\left(\mathbf{t}_{0}\right)\right]
$$

O valor $\mathbf{u}=0$ foi convenientemente ajustado para o início da sístole atrial. $\mathrm{O}$ atraso da ativação ventricular em relação à ativação atrial $\Delta \mathbf{T}_{\mathbf{a v}}$ foi dado no cálculo de $\varphi_{\mathbf{v}}(\mathbf{t})$, mantendo-se assim o mesmo adimensional u para as duas funções. Por isto, a Equação 3-23 deve ser reescrita,

\footnotetext{
${ }^{50}$ Conforme mencionado, foi escolhido para a sístole atrial um valor igual a um terço da duração da sístole ventricular.
} 


$$
\varphi_{\mathbf{v}}(\mathbf{t})= \begin{cases}\operatorname{sen}^{2}\left[\frac{\pi \mathbf{T}(\mathbf{t})}{\mathbf{T}_{\text {sis }, \mathbf{v}}(\mathbf{t})} \cdot \mathbf{u}\left(\mathbf{t}-\Delta \mathbf{T}_{\text {av }}\right)\right] & 0 \leq \mathbf{u}\left(\mathbf{t}-\Delta \mathbf{T}_{\text {av }}\right) \leq \frac{\mathbf{T}_{\text {sis }, \mathbf{v}}}{\mathbf{T}} \\ 0 & \frac{\mathbf{T}_{\text {sis, } \mathbf{v}}}{\mathbf{T}} \leq \mathbf{u}\left(\mathbf{t}-\Delta \mathbf{T}_{\mathbf{a v}}\right) \leq 1\end{cases}
$$

A Figura 3-23 a seguir mostra as funções de ativação resultantes.

A duração da sístole atrial $\mathbf{T}_{\text {sis,a }}$ decai linearmente com a freqüência cardíaca, da mesma forma que a ventricular, podendo-se escrever

$$
\mathbf{T}_{\text {sis }, \mathbf{a}}=\mathbf{T}_{\text {sis, }, \mathbf{a}, 0}-\mathbf{k}_{\text {sis }, \mathbf{a}} \frac{1}{\mathbf{T}}
$$

sendo $\mathbf{T}_{\text {sis,a, } 0}$ e $\mathbf{k}_{\text {sis,a }}$ parâmetros constantes. Note que $\mathbf{k}_{\text {sis,a }}$ não é o mesmo utilizado para os ventrículos.
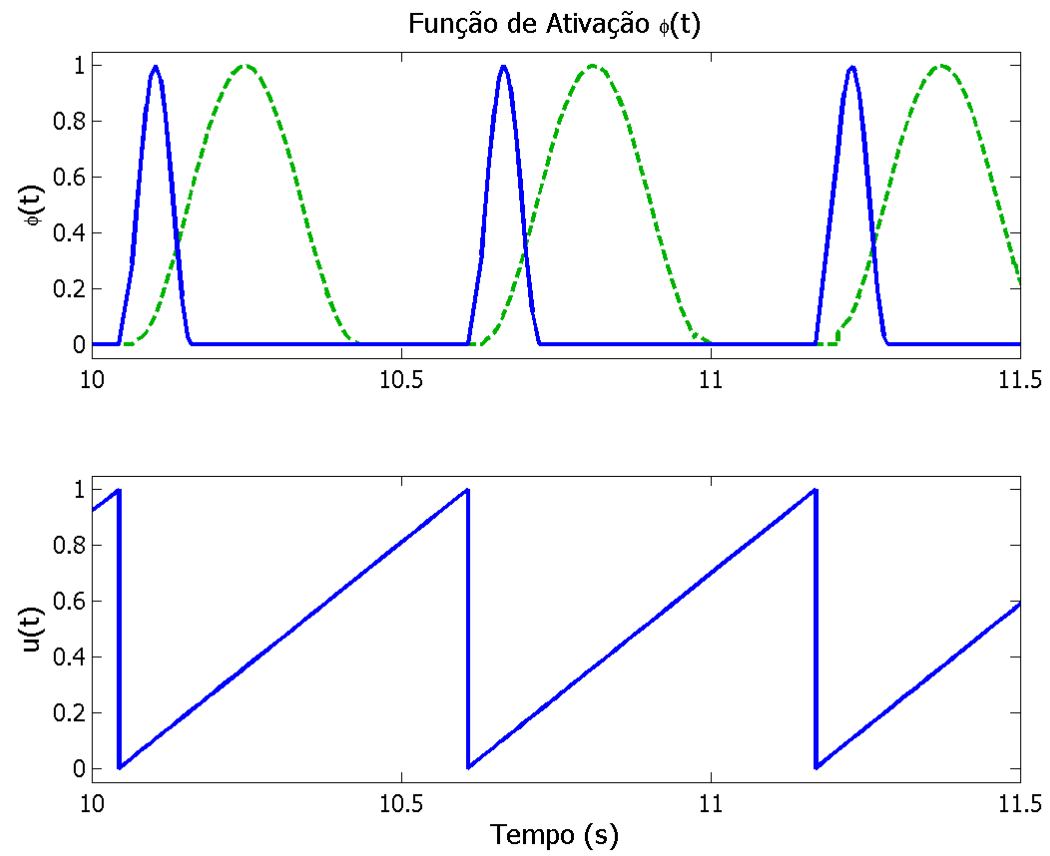

Figura 3-23 Função de ativação dos átrios $\varphi_{a}(t)$, em azul, e dos ventrículos $\varphi_{v}(t)$, em tracejado verde. Note que a fração do ciclo cardíaco $u(t)$ é a mesma para ambas.

As demais equações do lado esquerdo, referentes aos ventrículos, são mantidas as mesmas.

\section{Lado Direito}

São poucas as diferenças entre os lados direito e esquerdo, quase que restritas apenas aos valores dos parâmetros. De qualquer forma, serão apresentadas a seguir todas as equações relevantes à sua compreensão. 
Definindo $\dot{\mathbf{Q}}_{\text {dir,e }}$ como o fluxo de entrada no ventrículo direito (e conseqüentemente o fluxo de saída do átrio direito), e sendo $\dot{\mathbf{Q}}_{\mathbf{A D}}$ o fluxo de entrada no átrio direito, vindo das veias esplâncnicas e extra-esplâncnicas, pode-se escrever a conservação de massa de modo a se obter o volume no átrio direito $\mathbf{V}_{\mathbf{A D}}$ :

$$
\frac{\mathbf{d} \mathbf{V}_{\mathbf{A D}}}{\mathbf{d t}}=\dot{\mathbf{Q}}_{\mathrm{AD}}-\dot{\mathbf{Q}}_{\mathrm{dir}, \mathbf{e}}
$$

sendo $\dot{\mathbf{Q}}_{\mathbf{A D}}$ dado por

$$
\dot{\mathbf{Q}}_{\text {AD }}=\frac{\mathbf{P}_{\text {sv }}-\mathbf{P}_{\text {AD }}}{\mathbf{R}_{\text {sv }}}+\frac{\mathbf{P}_{\text {ev }}-\mathbf{P}_{\text {AD }}}{\mathbf{R}_{\text {ev }}}
$$

O fluxo de entrada no ventrículo direito, $\dot{\mathbf{Q}}_{\text {dir,e }}$, depende da abertura da válvula tricúspide, e pode ser descrito por

$$
\dot{\mathbf{Q}}_{\text {dir }, \mathbf{e}}= \begin{cases}0 & \text { se } \mathbf{P}_{\max , \mathbf{A D}} \leq \mathbf{P}_{\mathrm{VD}} \\ \frac{\mathbf{P}_{\mathrm{max}, \mathbf{A D}}-\mathbf{P}_{\mathrm{VD}}}{\mathbf{R}_{\mathbf{A D}}} & \text { se } \mathbf{P}_{\max , \mathbf{A D}}>\mathbf{P}_{\mathrm{VD}}\end{cases}
$$

onde $\mathbf{P}_{\max , \mathbf{A D}}$ é a função de elasticidade do átrio direito, e $\mathbf{R}_{\mathbf{A D}}$ é a respectiva resistência viscosa, considerada constante. A pressão instantânea no átrio direito $\mathbf{P}_{\mathbf{A D}}$ resulta da diferença entre a pressão isométrica e as perdas resistivas. Desta forma,

$$
\mathbf{P}_{\text {AD }}=\mathbf{P}_{\max , \mathbf{A D}}-\mathbf{R}_{\mathrm{AD}} \dot{\mathbf{Q}}_{\mathrm{dir}, \mathbf{e}}
$$

Por fim, pode-se escrever a relação pressão-volume do átrio direito:

$$
\begin{array}{r}
\mathbf{P}_{\text {max }, \mathbf{A D}}(\mathbf{t})=\varphi_{\mathbf{a}}(\mathbf{t}) \mathbf{E}_{\max , \mathbf{A D}}\left(\mathbf{V}_{\mathbf{A D}}-\mathbf{V}_{\mathbf{u}, \mathbf{A D}}\right)+\left[1-\varphi_{\mathbf{a}}(\mathbf{t})\right] \mathbf{E}_{\min , \mathbf{A D}}\left(\mathbf{V}_{\mathbf{A D}}-\mathbf{V}_{\mathbf{u}, \mathbf{A D}}\right) \\
0 \leq \varphi_{\mathbf{a}}(\mathbf{t}) \leq 1
\end{array}
$$

A função de ativação dos átrios $\varphi_{\mathbf{a}}(\mathbf{t})$, descrita pelas Equações 3-53 e 3-54, é a mesma para ambos os lados.

\subsubsection{Estimação de Parâmetros}

Visto que o modelo dos átrios não foi extraído de uma única fonte, ficou também inaplicável a obtenção dos valores para os parâmetros a partir de uma única fonte. A tentativa natural de adaptar os parâmetros sugeridos pelo único modelo disponível com átrios ativos, proposto por Goodwin et al. 
(2003), apresentou resultados inadequados. Foi então estabelecido como objetivo principal da estimação dos parâmetros a adequação dos valores simulados a valores teóricos encontrados na literatura. Apesar de esta escolha parecer evidente, conforme mencionado anteriormente, existe certa escassez de material relacionado à mecânica dos átrios. Além disso, os poucos trabalhos disponíveis, apresentam certa disparidade de resultados, não apenas quantitativos como também qualitativos. Por este motivo, a estimação foi feita com alvo principalmente em semelhanças qualitativas. Vale lembrar também que no presente trabalho não se pretendia realizar uma validação quantitativa do modelo ou de seus parâmetros, mas sim uma verificação qualitativa dos mesmos.

Os valores resultantes estão resumidos na Tabela 3-6 abaixo. Foram repetidos os parâmetros referentes aos ventrículos devido às alterações de símbolos ocorridas na descrição do modelo dos átrios. Os resultados obtidos através da simulação do modelo com a aplicação destes parâmetros são mostrados no capítulo seguinte.

Tabela 3-6 Parâmetros estimados para o modelo com átrios ativos.

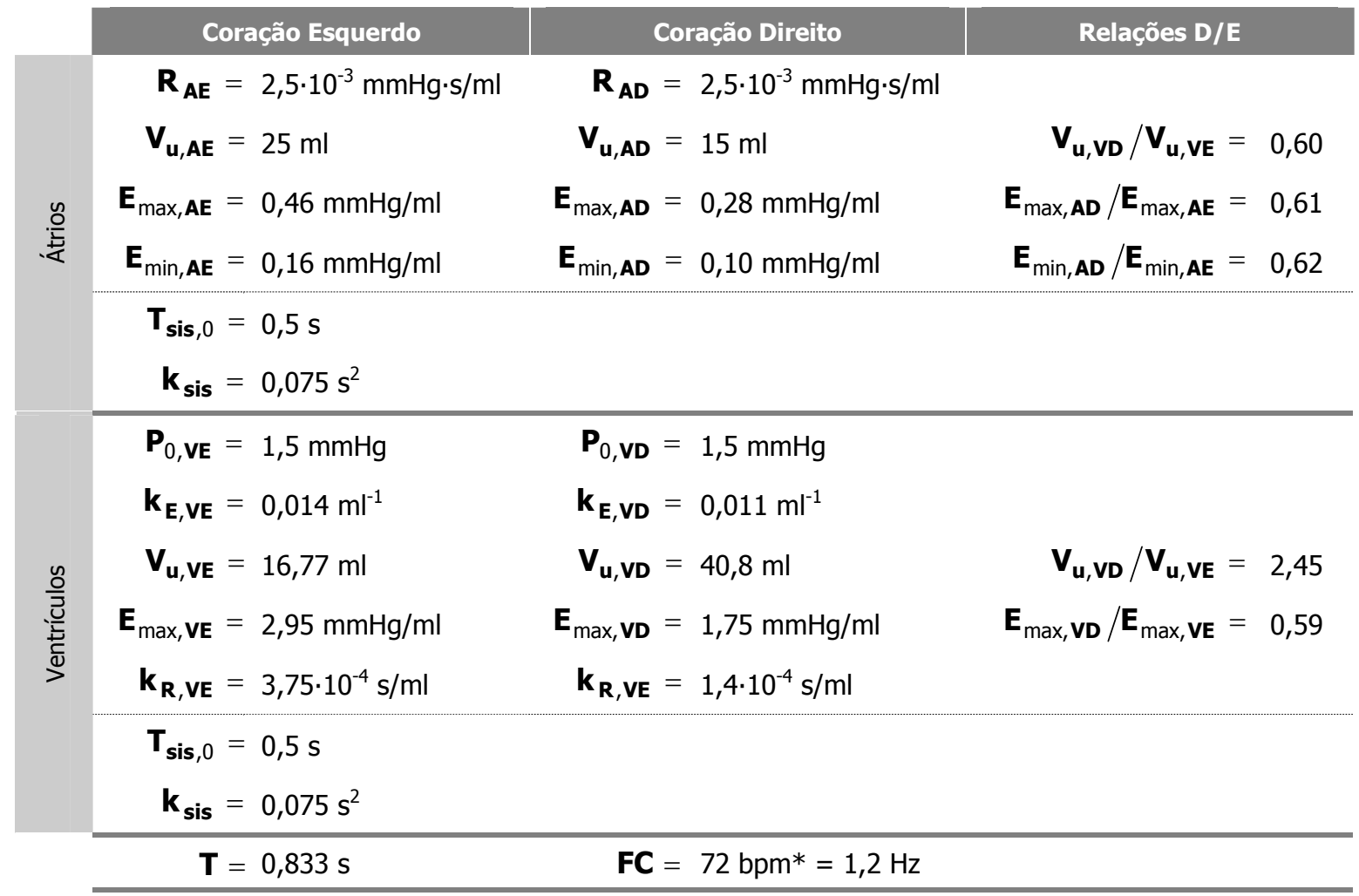

* bpm - batimentos por minuto

Os resultados das simulações realizadas são mostrados no Capítulo 4 a seguir. 


\section{Resultados das Simulações Numéricas}

Serão apresentados aqui alguns resultados obtidos através das simulações numéricas do modelo detalhado no Capítulo 3. Conforme será descrito, estas simulações foram realizadas em softwares comerciais, previamente ao desenvolvimento do simulador proprietário (descrito no próximo capítulo). O presente capítulo está organizado em duas grandes partes. Na primeira, são mostrados resultados referentes ao modelo sem os átrios ativos, reproduzido do trabalho de Ursino (1998). Na segunda, são exibidos os resultados obtidos com as simulações do modelo com átrios ativos. Ambas as partes são subdivididas em duas outras seções. Na primeira subseção foram selecionadas algumas curvas específicas, utilizadas no estudo de fisiologia e na avaliação da função cardíaca, com o intuito de verificar a adequação do modelo aos propósitos do simulador em desenvolvimento. Na segunda são mostrados os resultados referentes às simulações de alguns parâmetros individuais, a partir dos quais foram feitas considerações sobre o desempenho e a viabilidade da utilização do modelo em uma aplicação em tempo real.

\subsection{Malhas de Simulação}

O modelo completo, com átrios ativos, foi simulado numericamente nos softwares Matlab e Simulink. As equações foram colocadas diretamente no Simulink, por meio de diagramas de blocos, aproveitando-se dos solvers disponíveis no software. Foi utilizado o solver ode45 (Dormand-Prince) com intervalo de tempo variável. Para enfatizar as contribuições proporcionadas pela adição dos átrios ativos, em alguns momentos foram utilizados resultados do modelo com átrios ativos, reproduzido com base no trabalho de Ursino (1998), anteriormente ao desenvolvimento do modelo completo. Foram utilizados os mesmos procedimentos para as simulações deste modelo, incluindo o mesmo solver do Simulink.

O diagrama de blocos de nível mais alto, referente ao modelo com átrios ativos, é apresentado na Figura 4-1 abaixo, sendo que os cinco blocos visíveis consistem em agrupamentos de outros blocos menores (estes diagramas podem ser visualizados no Apêndice B). Neste nível é possível ob- 
servar as principais vias de tráfego de informação (alguns dos sinais, como phie Atuadores conduzem mais de um parâmetro simultaneamente). O diagrama de blocos correspondente para o modelo com átrios passivos é mostrado a seguir, na Figura 4-2.

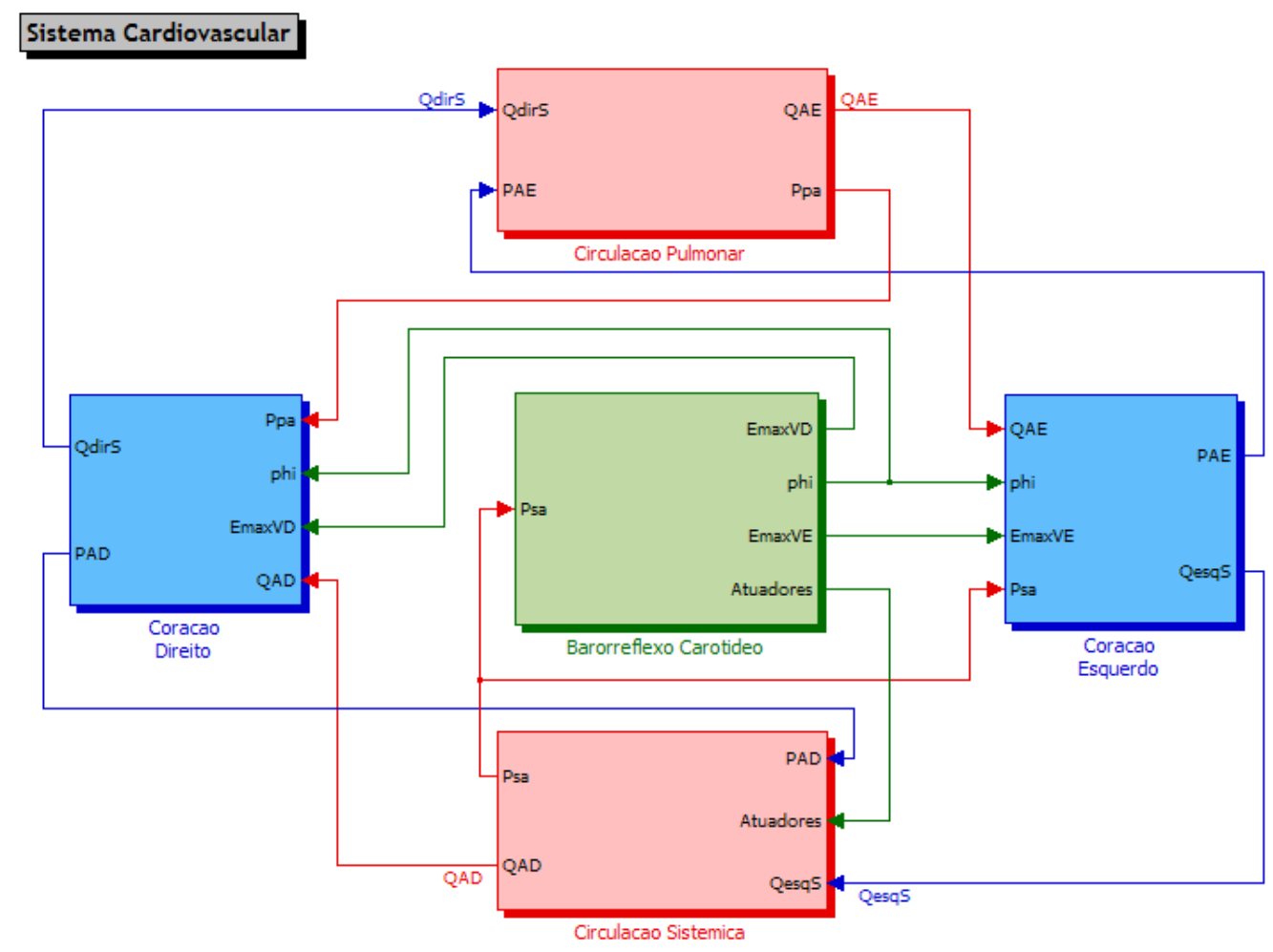

Figura 4-1 Malha de simulação em Simulink para o modelo com átrios ativos.

\section{Sistema Cardiovascular}

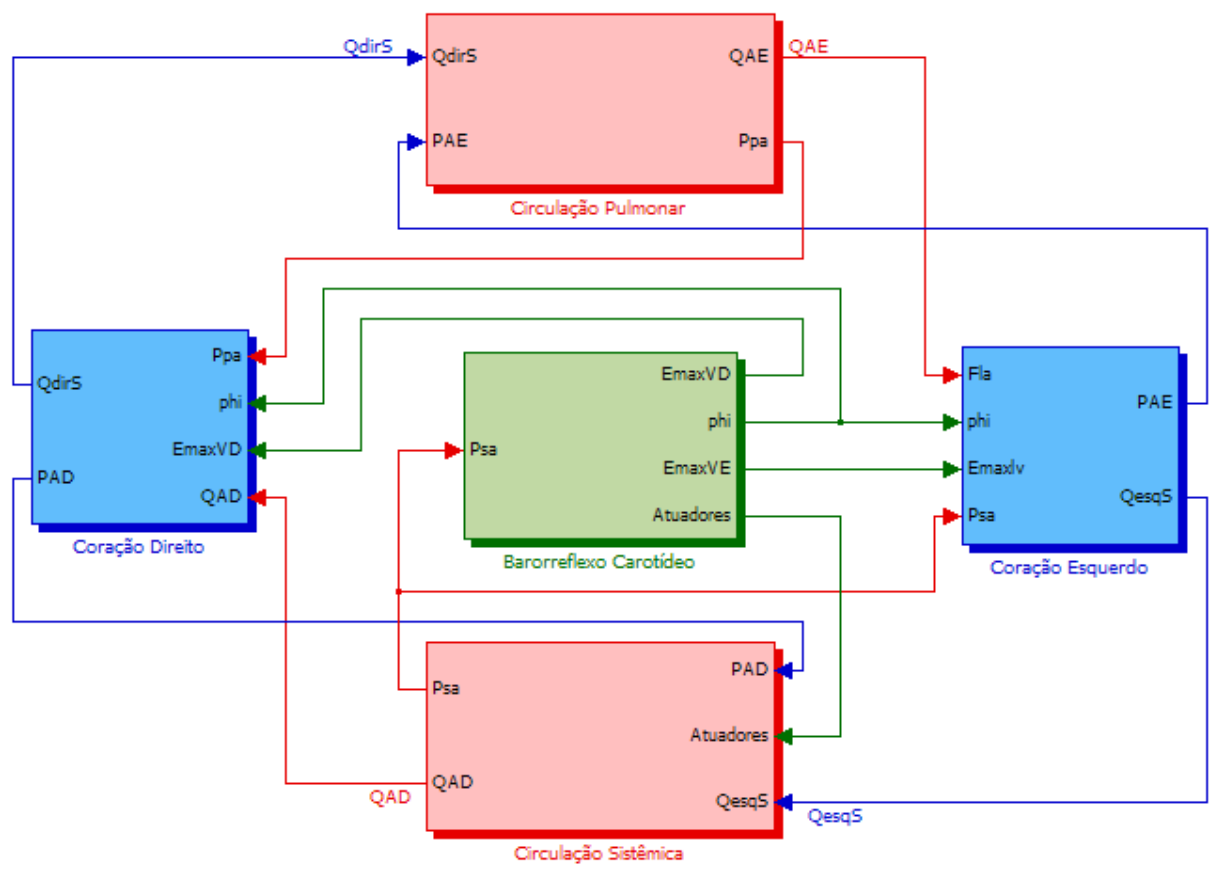

Figura 4-2 Malha de simulação em Simulink para o modelo com átrios passivos. 


\subsection{Simulações Numéricas}

Esta seção apresenta resultados obtidos a partir de diversas simulações numéricas realizadas com os modelos apresentados no Capítulo 3. A menção a "modelos" foi feita no plural pois serão apresentados resultados de ambos os modelos, com átrios passivos e com átrios ativos, quando a comparação for pertinente. O objetivo é comparar, de forma mais qualitativa, os resultados da simulação com curvas típicas disponíveis na literatura, de modo a avaliar o nível de adequação do modelo aos requisitos estabelecidos para o simulador. As comparações também serão estendidas aos resultados apresentados no trabalho de Ursino, para verificar os benefícios da inclusão dos átrios ativos. Para todas as situações simuladas foram consideradas situações de regime, com o sistema em malha fechada.

Para as comparações com dados fisiológicos reais foi utilizado o banco de dados PhysioBank, disponível no site PhysioNet ${ }^{1}$, ligado à Harvard Medical School e ao Massachussets Institute of Technology (MIT). O PhysioBank contém conjuntos de dados fisiológicos coletados em tempo real de pacientes em Unidades de Tratamento Intensivo (UTIs), servindo como referência para pesquisas na área biomédica. O banco de dados específico utilizado nas comparações deste trabalho foi o MIMIC, que contém informações obtidas de monitores multiparamétricos, incluindo curvas de pressão arterial sistêmica e pulmonar e de pressão venosa central (grande parte dos bancos de dados do PhysioBank contempla apenas informações de Eletrocardiografia). Dentro do MIMIC existem atualmente dados referentes a 72 pacientes (a meta divulgada para o projeto são 90 pacientes), sendo aqui utilizados apenas conjuntos contendo ao menos os três parâmetros de pressão citados (ABP, PAP e CVP²).

Os resultados estão organizados em tipos de parâmetros (como pressão, volume e débito cardíaco), em curvas de função cardíaca (tipicamente observadas em análises do sistema cardiovascular), e ainda em resultados da atuação do sistema de controle do reflexo baroceptor.

\footnotetext{
${ }^{1}$ O Physionet é um serviço pertencente ao Research Resource for Complex Physiologic Signals, sob a responsabilidade do National Center for Research Resources, do National Institutes of Health, do governo norte-americano. Consiste em um projeto cooperativo iniciado por pesquisadores do Beth Israel Deaconess Medical Center/Harvard Medical School de Boston, da Boston University, da McGill University, e do MIT.

${ }^{2}$ Estas são siglas comumente utilizadas em monitores de paciente, sendo que ABP significa Pressão Sangüínea Arterial (sistêmica), PAP significa Pressão Pulmonar Arterial e CVP significa Pressão Venosa Central.
} 


\subsubsection{Curvas de Pressão}

Os resultados apresentados a seguir correspondem a simulações realizadas com os parâmetros mostrados no Capítulo 3, conforme sugeridos por Ursino (1998). Em apenas alguns casos, que serão devidamente mencionados, certos parâmetros foram modificados para aproximar os resultados aos valores experimentais extraídos do PhysioBank. Foram consideradas, para os gráficos aqui expostos, situações de regime, com o sistema em malha fechada.

\section{Pressões Arteriais}

A Figura 4-3 abaixo mostra uma simulação das pressões ventricular esquerda e arterial sistêmica, obtidas a partir do modelo com átrios passivos apresentado na Seção 3.1, em uma situação de regime (após 50 segundos de acomodação, com condições iniciais nulas). A efeito de comparação, foi colocado à direita da figura um gráfico extraído diretamente do trabalho de Ursino (1998). Note que, apesar de os parâmetros utilizados na simulação numérica em Matlab serem idênticos aos valores declarados no artigo citado, a amplitude das curvas é diferente. Isso pode ser devido (1) à utilização, no trabalho de Ursino, de parâmetros diferentes dos mencionados nas tabelas, ou (2) a duas situações de regime diferentes, em momentos distintos da atuação do barorreflexo.
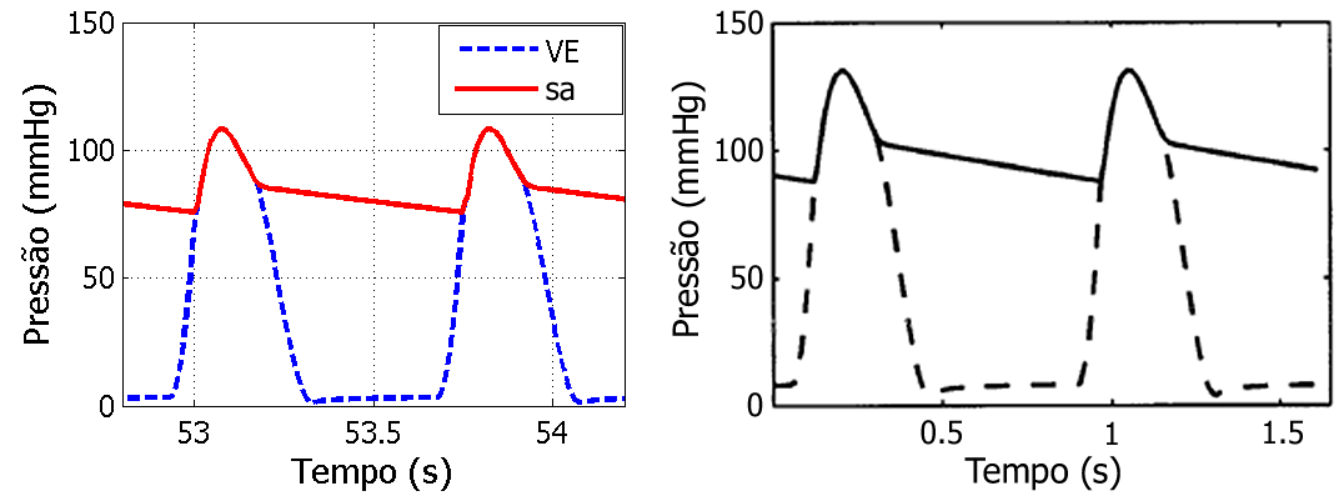

Figura 4-3 Simulação do modelo com átrios passivos. 0 gráfico da esquerda mostra os resultados da simulação em Matlab do modelo apresentado na Seção 3.1, com os parâmetros extraídos do trabalho de Ursino (1998). 0 gráfico da direita foi extraído diretamente do trabalho citado.

Uma comparação com valores obtidos do banco de dados MIMIC 055/055 do PhysioBank mostra significativa aderência do modelo (átrios passivos) aos valores experimentais. A Figura 4-4 abaixo apresenta uma simulação lado a lado com um gráfico do paciente real. Apesar da pequena diferença na freqüência cardíaca e da ausência do segundo pico de pressão, nota-se importante semelhança nos valores de amplitude. 

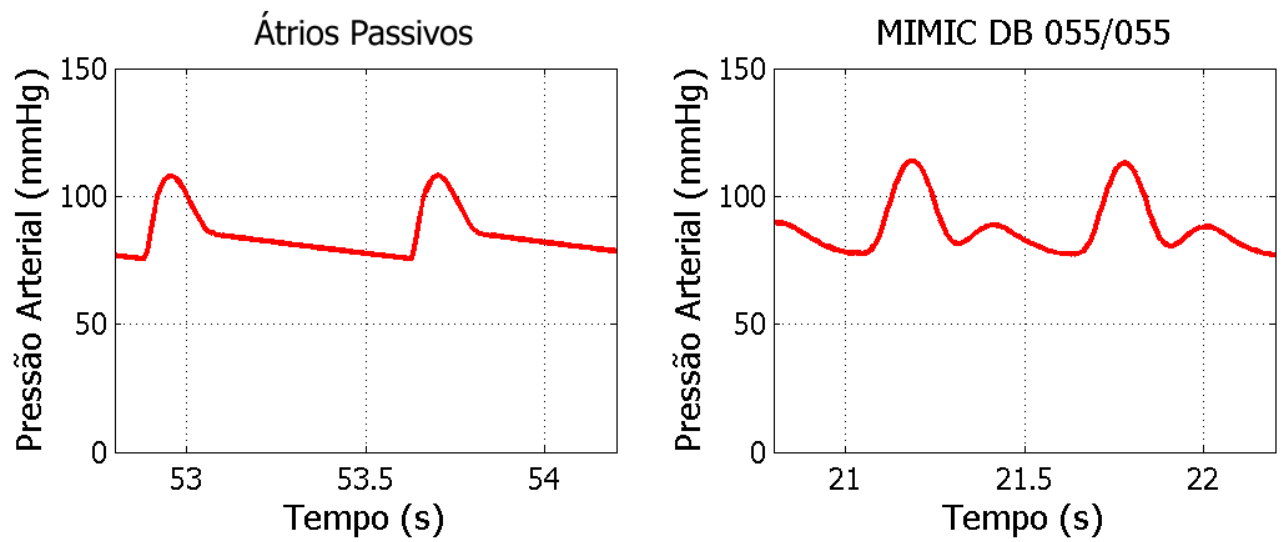

Figura 4-4 Comparação da pressão arterial sistêmica do modelo com átrios passivos e do paciente 055 do MIMIC.

O modelo com átrios ativos, mostrado à direita da Figura 4-5 abaixo, acrescenta, no caso da pressão sistêmica arterial, apenas uma sutil variação na amplitude dos valores, como já era esperado (a inclusão da atividade dos átrios reflete mais significativamente nos compartimentos cardíacos). No entanto, um aumento do parâmetro de inércia das artérias sistêmicas resulta em uma significativa aproximação aos valores reais, conforme mostrado na Figura 4-6 a seguir, onde nota-se o aparecimento dos picos de menor amplitude. Foi utilizado um valor de $\mathbf{L}_{\text {sa }}$ igual a $0.001 \mathrm{mmHg} \cdot \mathrm{ml} / \mathrm{s}^{2}$, ao invés dos $0.00022 \mathrm{mmHg} \cdot \mathrm{ml} / \mathrm{s}^{2}$ sugeridos por Ursino (1998).
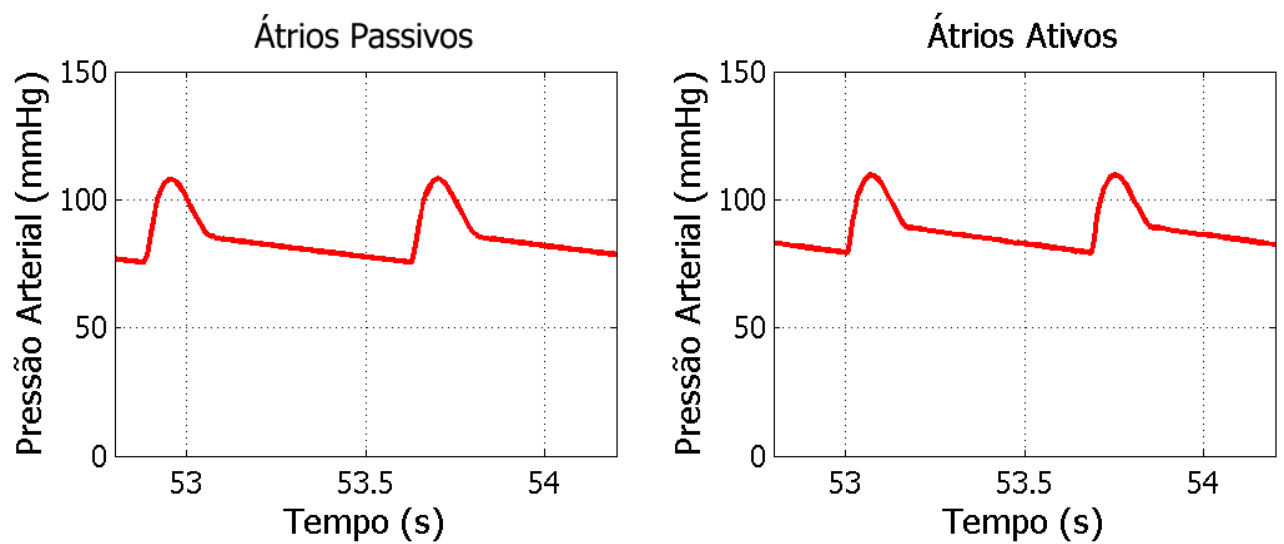

Figura 4-5 Comparação da simulação da pressão arterial sistêmica dos modelos com átrios passivos e com átrios ativos. 


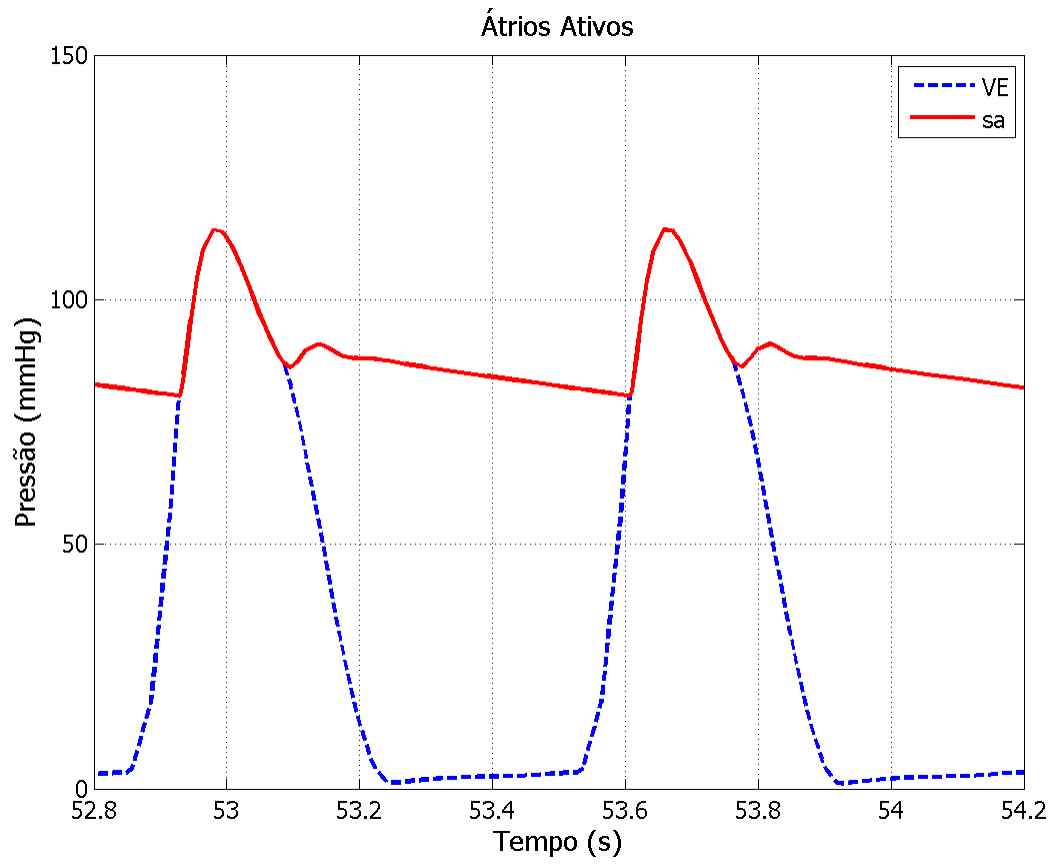

Figura 4-6 Pressões no ventrículo esquerdo e nas artérias sistêmicas para o modelo com átrios ativos, com valor de inércia aumentado (para $0.001 \mathrm{mmHg} \cdot \mathrm{ml} / \mathrm{s}^{2}$ ).

Os resultados para a pressão pulmonar arterial não se mostraram tão próximos dos valores reais quanto os da pressão sistêmica. A Figura 4-7 abaixo mostra uma comparação entre a simulação do modelo com átrios ativos e o paciente 231 do MIMIC. Note que os valores simulados estão significativamente afastados dos valores reais, restando uma semelhança apenas qualitativa, suficiente para a aplicação desejada. É provável que estas diferenças possam ter sido provocadas pela escolha dos parâmetros do modelo, ou mesmo por decisões feitas na modelagem, como a consideração de resistência constante nos átrios ativos. De qualquer forma, estas diferenças não influem no comportamento global do sistema.
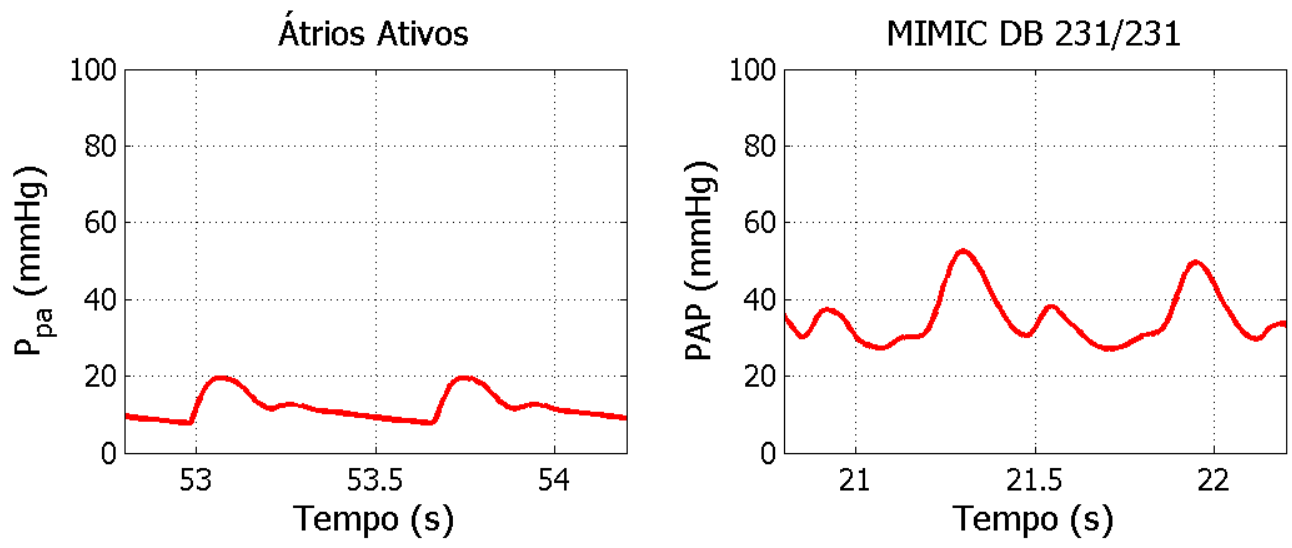

Figura 4-7 Comparação entre a pressão pulmonar arterial simulada com o modelo com átrios ativos e o banco de dados MIMIC 231/231. Foram mantidos os símbolos originais, tanto do modelo, na figura da esquerda, quanto do paciente (símbolo usual no meio médico), apesar de ambos representarem a mesma pressão. 
Diversas combinações de parâmetros foram testadas para aproximar os valores simulados dos valores reais, mas todas resultaram em algum compromisso em outro parâmetro. A única alteração utilizada na simulação acima foi novamente a inércia arterial $\mathbf{L}_{\mathbf{p a}}$, passada de 0.00018 para 0.0007 $\mathrm{mmHg} \cdot \mathrm{ml} / \mathrm{s}^{2}$ - o que provocou o surgimento dos picos de pressão de intensidade menor observados na Figura 4-7 acima.
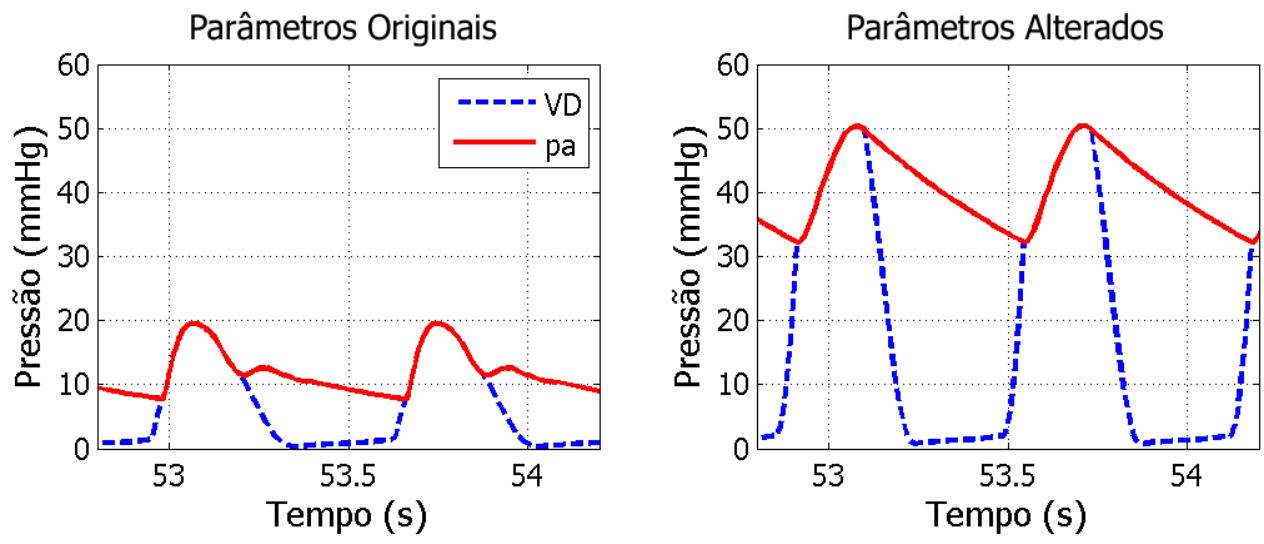

Figura 4-8 Exemplo de alteração de parâmetros inadequada, provocando deformações na pressão ventricular direita. Os parâmetros do gráfico da esquerda são os mesmos sugeridos por Ursino (com exceção da indutância), ou seja, $C_{p a}=0.76 \mathrm{ml} / \mathrm{mmHg}, R_{p a}=0.023 \mathrm{mmHg} \cdot \mathrm{s} / \mathrm{ml} \mathrm{e} \mathrm{L}_{\mathrm{pa}}=0.0007 \mathrm{mmHg} \cdot \mathrm{ml} / \mathrm{s}^{2}$. No gráfico da direita foram utilizados $C_{p a}=1.76 \mathrm{ml} / \mathrm{mmHg}, R_{p a}=0.45 \mathrm{mmHg} \cdot \mathrm{s} / \mathrm{ml}$ e $L_{p a}=0.0018$ $\mathrm{mmHg} \cdot \mathrm{ml} / \mathrm{s}^{2}$.

A Figura 4-8 acima mostra os resultados de um dos testes de variação de parâmetros, que apesar de ter corrigido a intensidade da pressão pulmonar arterial, acabou provocando enorme deformação na curva de pressão ventricular.

\section{Pressão Venosa Central}

Uma última comparação com dados de monitoração do MIMIC pode ser feita para a pressão venosa central $(\mathrm{CVP})^{3}$, medida nas veias imediatamente anteriores ao átrio direito. A Figura 4-9 abaixo compara o resultado obtido para a pressão esplâncnica venosa no modelo com átrios ativos com uma curva obtida da monitoração da pressão venosa central do paciente 474 do MIMIC. Note que a semelhança de forma entre as curvas permanece, como na pressão pulmonar arterial, mas aqui a diferença de amplitude é bastante acentuada. Os parâmetros de simulação utilizados foram os mes-

\footnotetext{
${ }^{3}$ Ambas as medições de CVP e de PAP são feitas com cateteres de Swan-Ganz (ou cateteres de artéria pulmonar), comumente utilizados na determinação do débito cardíaco e da pressão de oclusão da artéria pulmonar.
} 
mos utilizados por Ursino (1998), com exceção das resistências venosas esplâncnica $\left(\mathbf{R}_{\text {sv }}\right)$ e extraesplâncnica ( $\mathbf{R}_{\mathbf{e v}}$ ) - que foram alteradas, respectivamente, de 0.038 para $0.060 \mathrm{mmHg} \cdot \mathrm{s} / \mathrm{ml}$, e de 0.016 para $0.030 \mathrm{mmHg} \cdot \mathrm{s} / \mathrm{ml}$. Estas alterações proporcionaram um pequeno aumento de amplitude $\mathrm{e}$ uma maior aproximação no formato da curva.
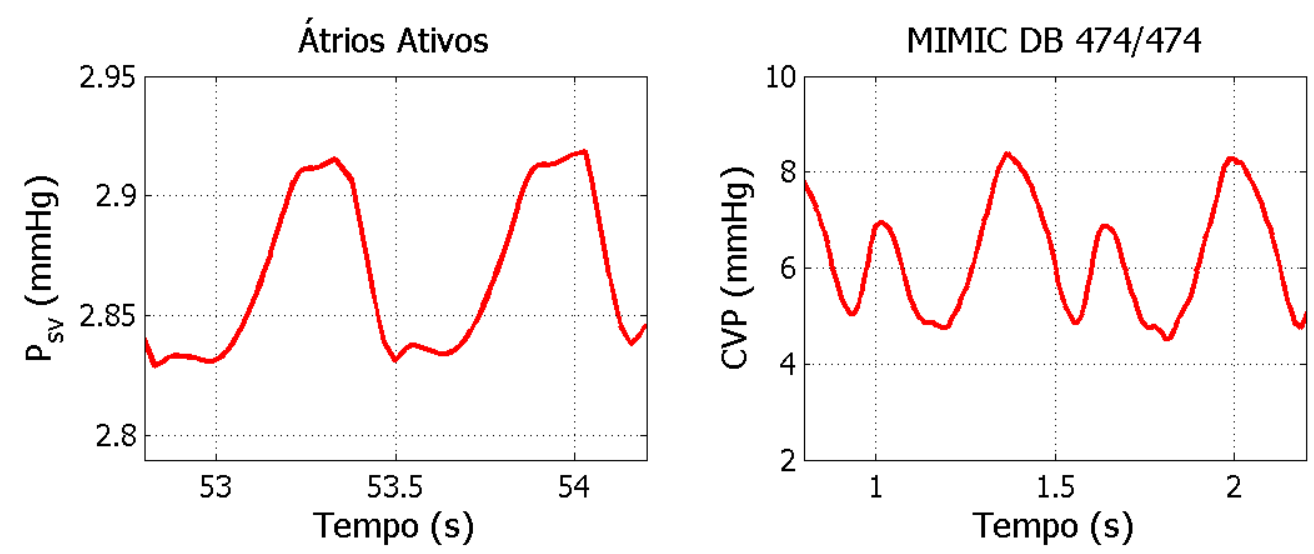

Figura 4-9 Comparação entre a pressão venosa central simulada com o modelo com átrios ativos e o banco de dados MIMIC 474/474.

\section{Pressões Cardíacas}

Os próximos resultados referem-se às pressões internas ao coração, para as quais não foram encontrados dados de monitoração de pacientes reais, e nem mesmo figuras de referência no trabaIho de Ursino (1998). As comparações aqui serão feitas diretamente entre os modelos, com átrios passivos e com átrios ativos, para ressaltar as contribuições proporcionadas pelo último. Quando disponíveis, serão utilizadas figuras extraídas da literatura para maior embasamento teórico.
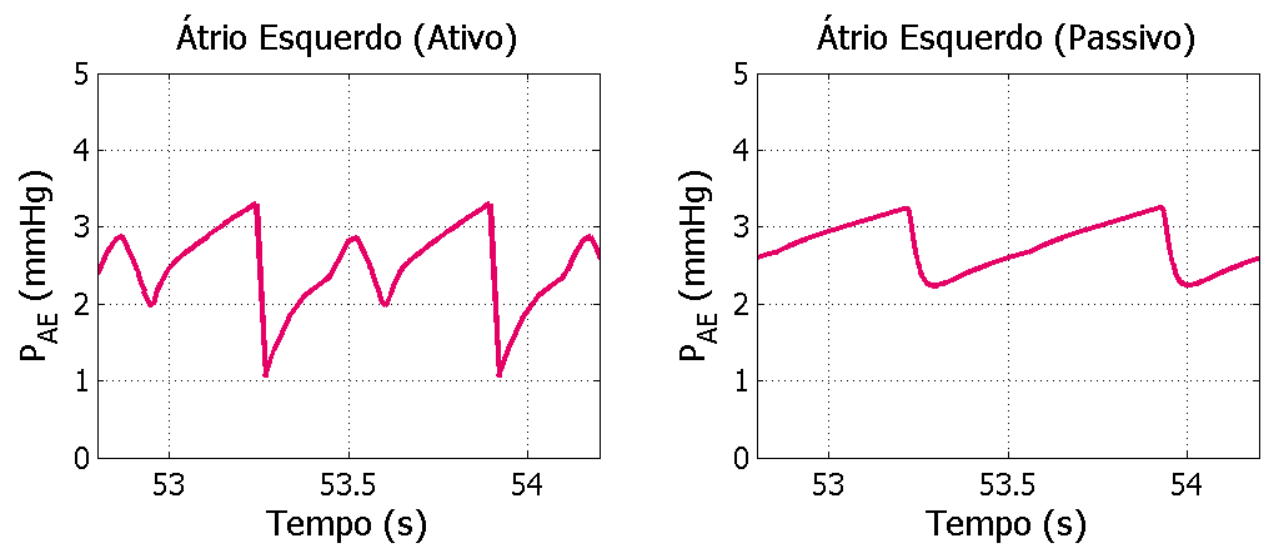

Figura 4-10 Comparação entre a pressão atrial esquerda no modelo com átrios ativos (à esquerda) e com átrios passivos (à direita). 
A Figura 4-10 acima mostra uma comparação entre a pressão atrial esquerda nos modelos com átrios passivos e ativos. Evidentemente, há uma enorme diferença entre as duas curvas, devido à inclusão da atividade contrátil. Pode-se notar, na figura da esquerda (átrios ativos), a formação das ondas características do traçado de pressão atrial, vistas na Figura 4-11 abaixo. Conforme mencionado anteriormente, pode-se notar que, por se tratar de uma medição de difícil acesso, há variação mesmo entre os valores experimentais, inclusive de caráter qualitativo (variações de amplitude seriam normais). Percebe-se, no entanto, que persistem algumas diferenças fundamentais entre os valores simulados e os valores experimentais, que não puderam ser eliminadas mesmo após alguns testes de variação de parâmetros. O formato da curva simulada aproxima-se mais da curva inferior da Figura 4-11, extraída de Pagel et al. (2003), com a amplitude da onda a menor que a da onda v. A onda $c$ simulada aparece menos pronunciada que a mostrada por Pagel et al., mas também não se assemeIha à onda $c$ vista em Matsuda et al. (1983), no traçado superior da figura. Ainda em relação ao formato da curva, percebe-se que a queda de pressão após a onda $v$ é bastante acentuada no traçado simulado, diferentemente dos experimentais. Por fim, pode-se notar uma grande diferença entre as amplitudes das curvas - os resultados da simulação mostram valores entre 1 e $4 \mathrm{mmHg}$, enquanto os dados experimentais ficam entre 6 e $14 \mathrm{mmHg}$. Ainda não é possível afirmar que estas diferenças sejam devidas a erros no modelo; o mais provável é que uma sintonia nos parâmetros possa vir a resolvê-las, visto que os parâmetros utilizados foram extraídos de um trabalho externo.

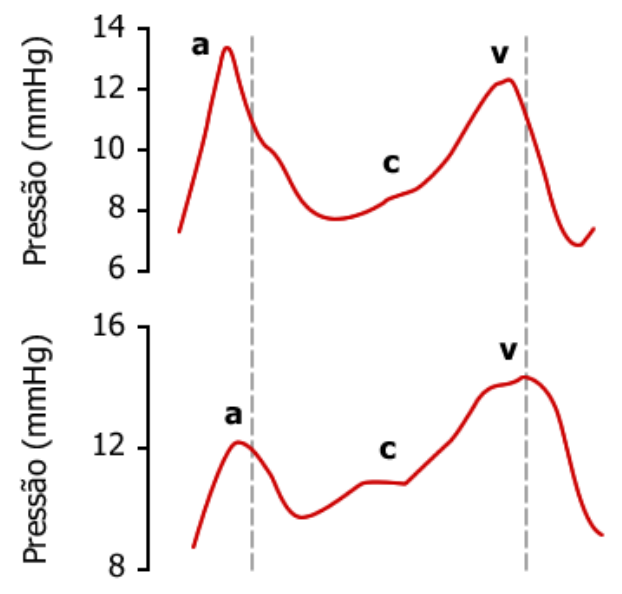

Figura 4-11 Traçados experimentais de pressão atrial esquerda, adaptados, respectivamente de cima para baixo, de Matsuda et al. (1983) e de Pagel et al. (2003).

A Figura 4-12 abaixo mostra uma comparação similar para o átrio direito. Novamente, nota-se uma grande variação entre as curvas, devido à contratilidade incluída no modelo com átrios ativos. 

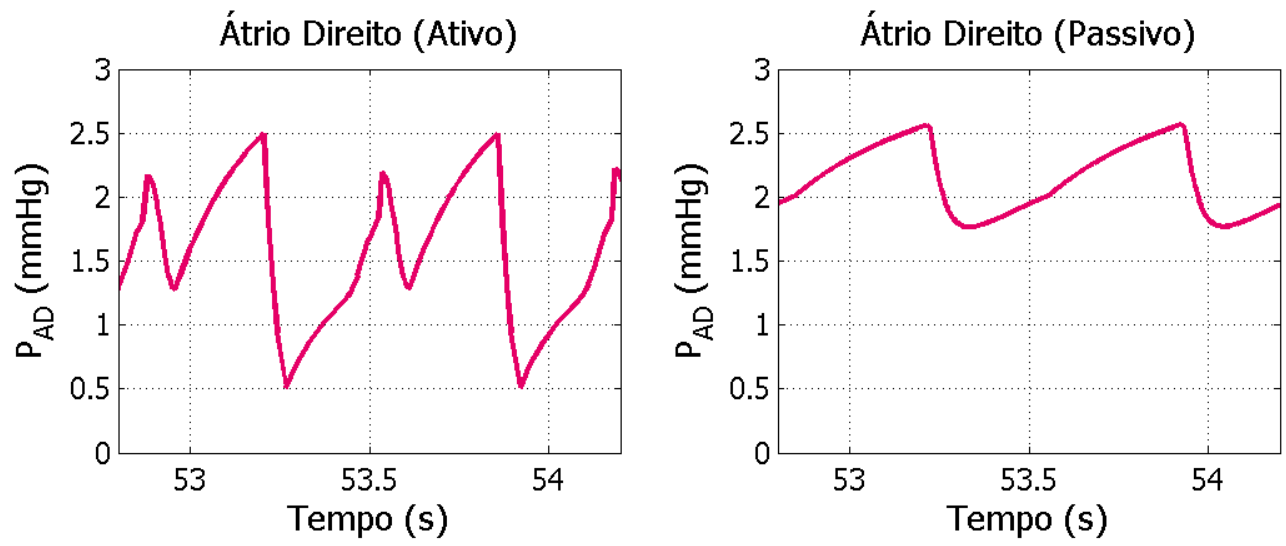

Figura 4-12 Comparação entre a pressão atrial direita no modelo com átrios ativos (à esquerda) e com átrios passivos (à direita).

Como é possível observar na Figura 4-13 abaixo, as considerações feitas para o átrio esquerdo permanecem válidas para o lado direito. Vale ressaltar novamente a variação de valores nos dados experimentais, que ocorre mesmo com um parâmetro de acesso mais fácil (a pressão no átrio direito é comumente medida através do procedimento de Swan-Ganz, amplamente utilizado em hospitais). Observam-se assim as semelhanças entre a curva simulada e as experimentais. Quanto às amplitudes, os valores simulados assemelham-se mais ao traçado experimental de Maniar et al. (2003), na parte inferior esquerda da Figura 4-13. No caso do formato, a semelhança é maior com relação aos resultados do trabalho de Miller et al. (1986), que exibe transições mais suaves entre as ondas $a$, ce $v$. Percebe-se que para o átrio direito os valores extremos da curva simulada se aproximaram consideravelmente dos experimentais - os resultados da simulação ficaram entre 0.5 e $2.5 \mathrm{mmHg}$, enquanto os experimentais mostram-se entre 2.5 e $7.5 \mathrm{mmHg}$.

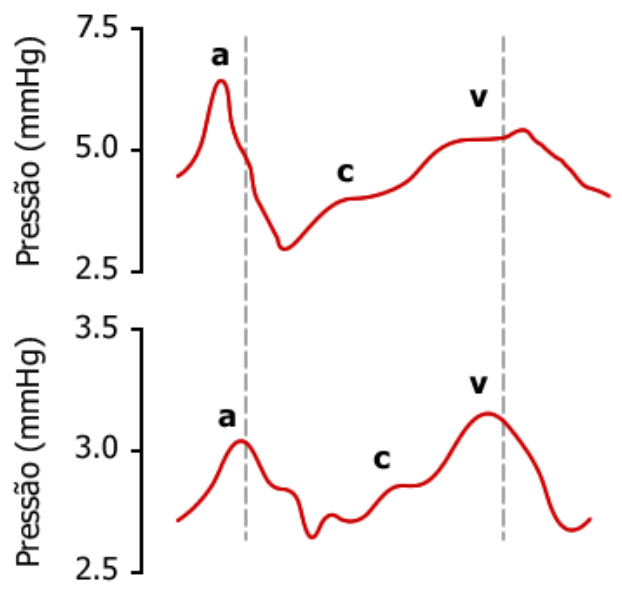

Figura 4-13 Traçados experimentais de pressão no átrio direito, adaptados, respectivamente de cima para baixo, de Miller et al. (1986) e de Maniar et al. (2003). 
O traçado de pressão ventricular esquerda já se mostrava satisfatório no modelo com átrios passivos, mantendo-se praticamente o mesmo após a inclusão da atividade atrial contrátil. Isso pode ser visto na Figura 4-14 abaixo, onde nota-se apenas uma pequena elevação de pressão máxima no caso dos átrios ativos.
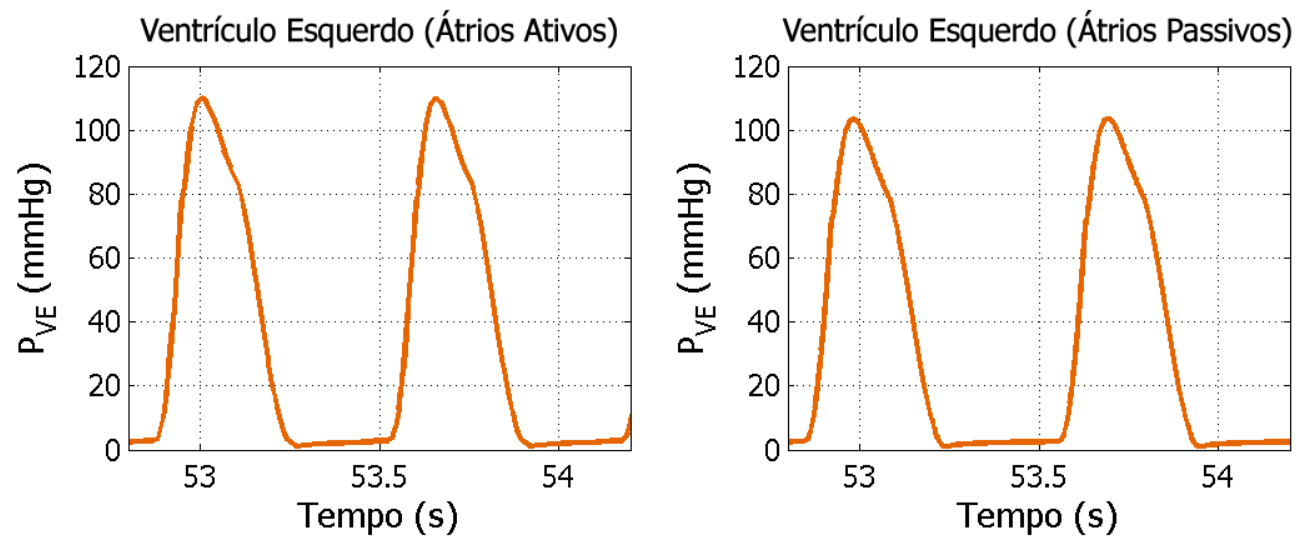

Figura 4-14 Comparação entre os traçados de pressão no ventrículo esquerdo resultantes da simulação do modelo com átrios ativos (à esquerda) e com átrios passivos (à direita).

Uma comparação com dados da literatura mostra que estes resultados estão bastante aderentes com a teoria, conforme é possível observar na Figura 4-15 abaixo. Como diferenças, podem-se ressaltar o formato mais pontudo da curva simulada e a aparente ausência da curva $a$ da pressão atrial. Conforme comentado anteriormente, os valores de pressão atrial ficaram muito abaixo dos valores teóricos, fazendo com que a curva pareça reta.

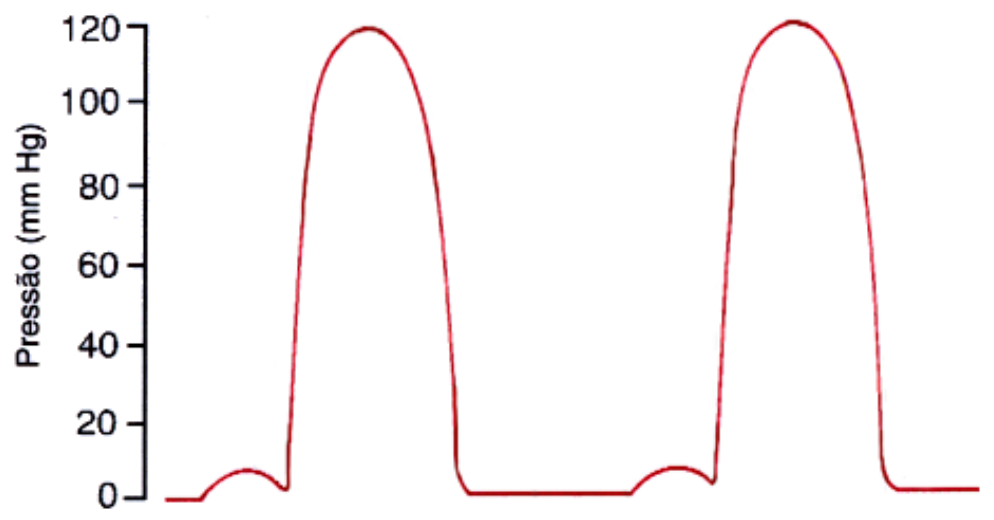

Figura 4-15 Curva de pressão ventricular esquerda adaptada de Guyton e Hall (1996). O período do ciclo foi estimado entre 0.8 e $1 \mathrm{~s}$, a partir de comentários dos autores.

O detalhe apresentado na Figura 4-16 mostra a forma da onda a no traçado da pressão ventricular. Note que a elevação de fato ocorre, mas possui uma amplitude bastante reduzida, devido à baixa amplitude da pressão atrial. 


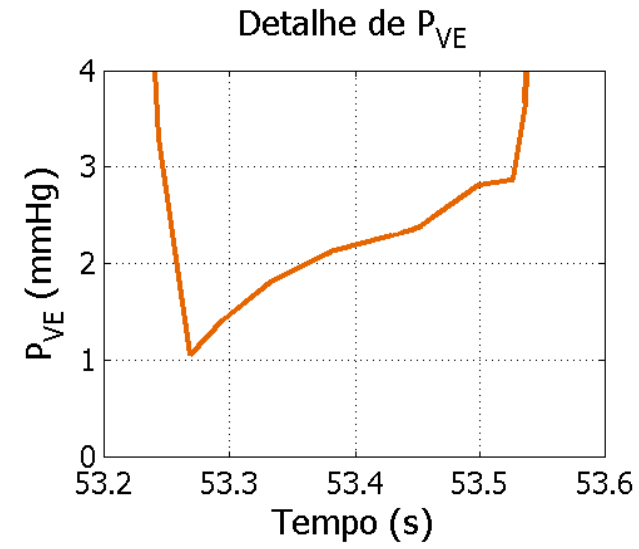

Figura 4-16 Ampliação da região em que aparece a elevação causada pela onda $a$ da pressão atrial no traçado de pressão ventricular.

No caso do ventrículo direito, a diferença entre a pressão simulada no modelo com átrios ativos e no modelo com átrios passivos é mais pronunciada, como mostra a Figura 4-17 abaixo. A comparação com dados da literatura mostra que a adição da atividade contrátil aos átrios tornou o modelo mais realista. Os valores extremos de pressão estão dentro das faixas de normalidade comumente recomendadas para a monitoração de pacientes - os valores mínimos variam entre 0 e $8 \mathrm{mmHg}$ e os valores máximos vão de 15 a 25 mmHg (QUICK..., 2002).
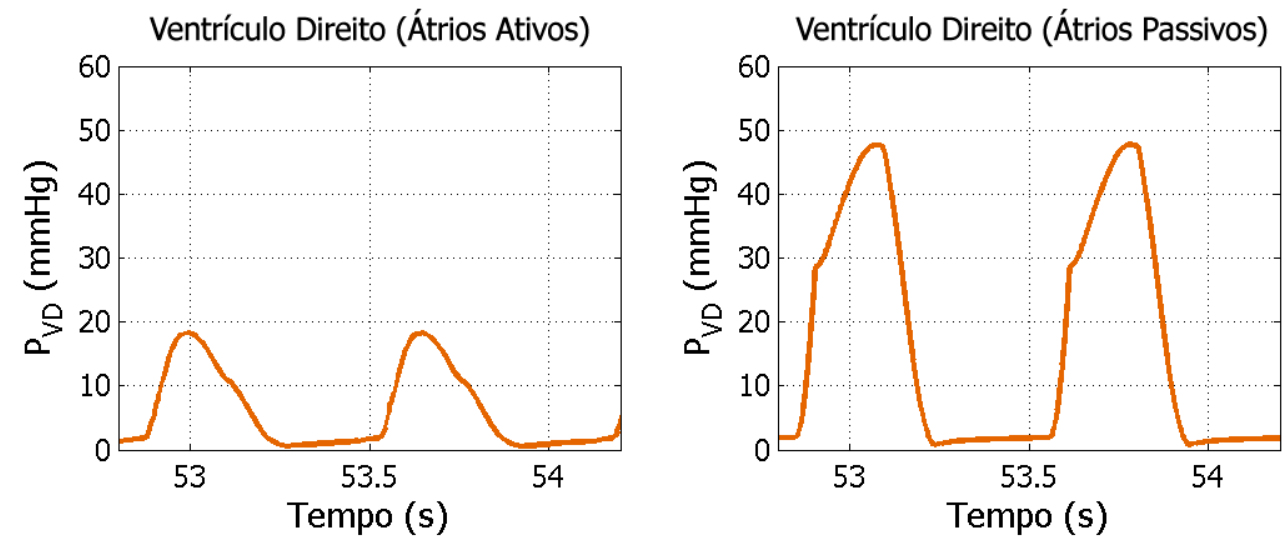

Figura 4-17 Comparação entre a pressão no ventrículo direito originada da simulação do modelo com átrios ativos (à esquerda) e passivos (à direita).

Ainda em comparação com dados teóricos, a Figura 4-18 mostra um conjunto de curvas extraído de Antoni (1996b), onde se nota semelhança de forma e amplitude com a simulação do modelo com átrios ativos. Assim como do lado esquerdo, a elevação de pressão ventricular devida à onda $a$ da pressão atrial é pouco aparente, por causa de sua baixa amplitude. 


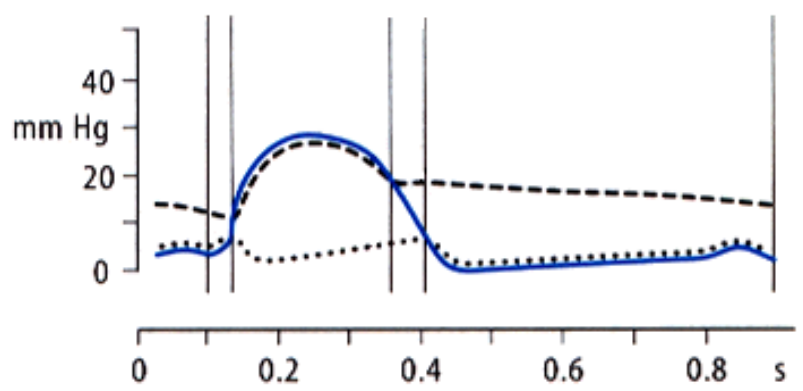

Figura 4-18 Pressões no lado direito do coração. A linha azul representa a pressão no ventrículo direito. Podem-se notar, através da linha pontilhada, as ondas $a, c$ e $v$ da pressão atrial, e a influência da primeira delas na pressão ventricular (adaptado de Antoni, 1996b).

\subsubsection{Curvas de Volume}

Por serem de difícil determinação prática, as medições de volume não são comumente utilizadas em ambientes clínicos. De forma experimental, a maior parte dos trabalhos consultados menciona apenas o volume nas câmaras cardíacas. Adicionalmente, pode-se afirmar que as contribuições mais significativas para as curvas de volume, em decorrência da adição da atividade contrátil aos átrios, podem ser percebidas nos compartimentos do coração. Considera-se novamente o sistema em malha fechada e em regime.

Mantendo a mesma seqüência de compartimentos utilizada na apresentação dos resultados de pressão, a Figura 4-19 abaixo mostra uma comparação entre o volume atrial esquerdo simulado no modelo com átrios ativos e no modelo com átrios passivos.
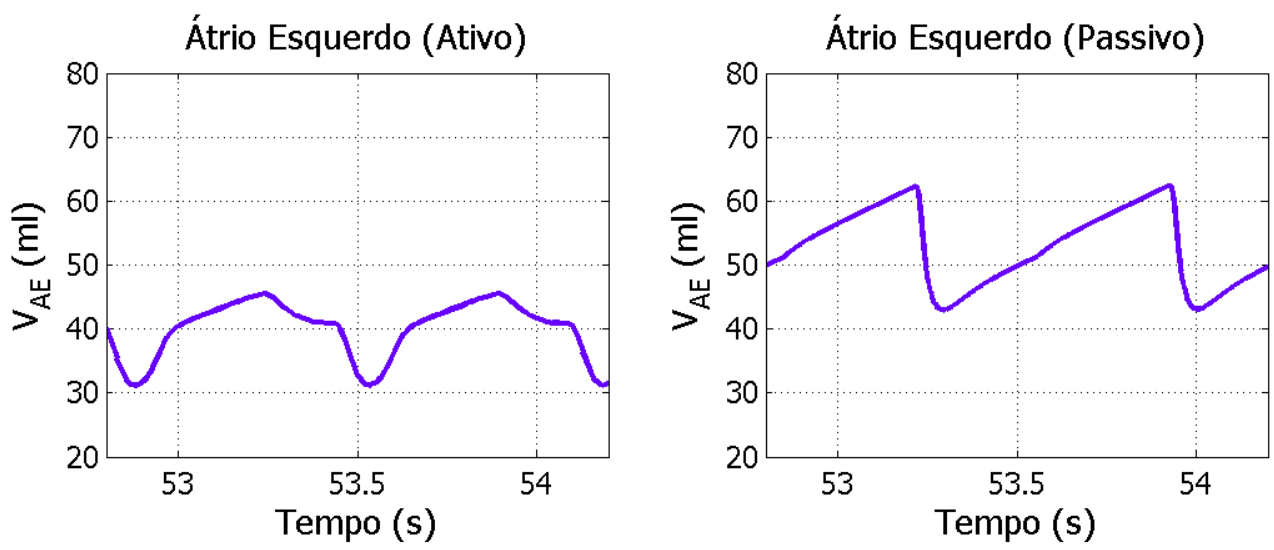

Figura 4-19 Comparação entre o volume atrial esquerdo no modelo com átrios ativos (à esquerda) e com átrios passivos (à direita).

Percebe-se que a inclusão da atividade dos átrios foi responsável por tornar o formato da curva mais fisiológico, conforme visto no traçado inferior da Figura 4-20. Mais uma vez, podem-se repetir as considerações sobre a variabilidade dos dados experimentais, que no caso do volume, é agravada 
pelas imprecisões inerentes aos métodos de medição. Desta forma, a variação das amplitudes pode ser considerada satisfatória. A diferença de fase entre as duas curvas não deve ser considerada, pois os momentos simulados são arbitrários, não havendo relação entre eles.

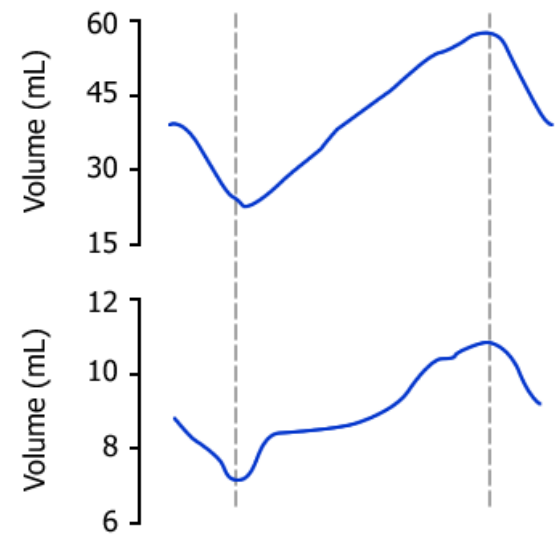

Figura 4-20 Traçados experimentais de volume atrial esquerdo. 0 traçado superior foi adaptado de Matsuda et al. (1983), e o inferior, de Pagel et al. (2003).

Os resultados de volume atrial direito são apresentados na Figura 4-21 abaixo. Note a enorme variação entre as amplitudes das duas curvas, além de sua grande diferença de forma. Percebe-se também a formação mais pronunciada de um platô (que também aparecera na curva do átrio esquerdo) durante o esvaziamento passivo do átrio, quando o sangue passa diretamente das veias para o ventrículo, mas ainda antes da contração atrial.
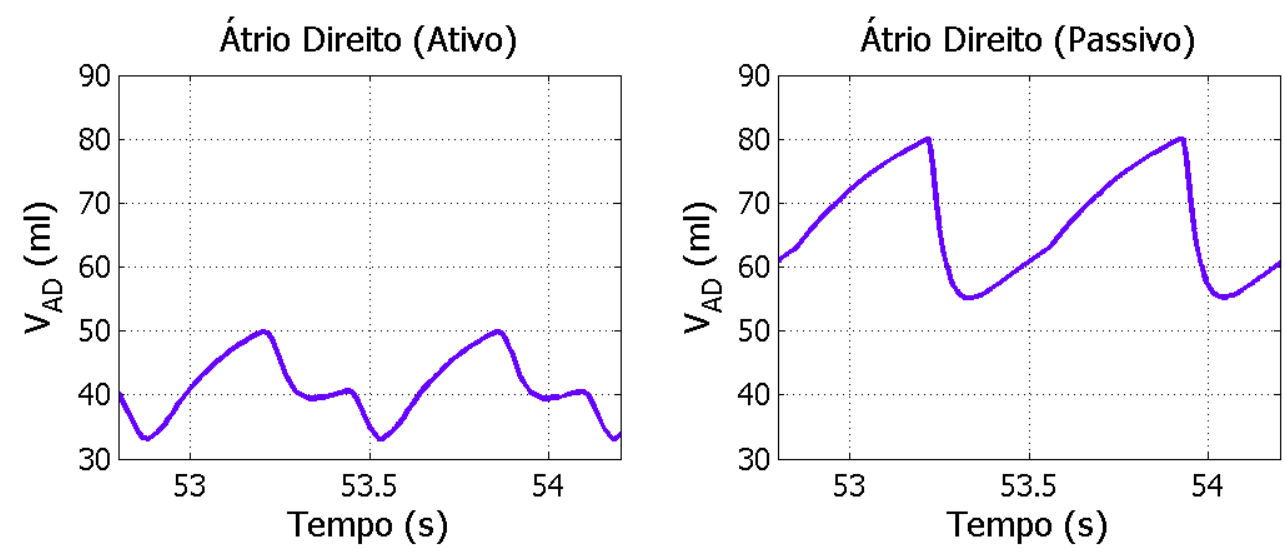

Figura 4-21 Comparação entre os resultados de volume atrial direito calculados a partir do modelo com atividade atrial (à esquerda) e do modelo sem atividade atrial (à direita).

Em comparação com curvas obtidas experimentalmente, mostradas na Figura 4-22 abaixo, observa-se uma maior proximidade quantitativa, além de uma fase de enchimento atrial (onde ocorre a onda $v$ de pressão) bastante semelhante. No entanto, não ocorre nestas curvas o platô mencionado acima. 


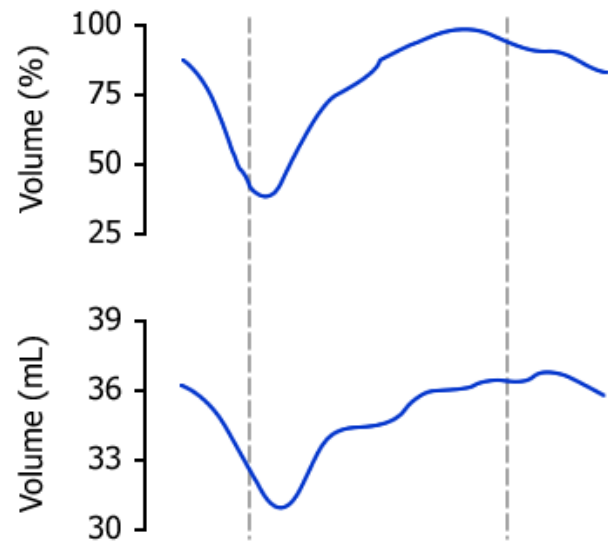

Figura 4-22 Curvas de volume no átrio direito, obtidas experimentalmente por Maniar et al. (2003) e por Miller et al. (1986).

O traçado de volume no ventrículo esquerdo, mostrado na Figura 4-23, sofreu melhoria significativa com a inclusão da contratilidade dos átrios. Aparece, agora, a curva decorrente da contração atrial, que proporciona um maior enchimento do ventrículo antes da sístole. A curva de volume teórica pode ser vista na Figura 4-24, extraída de Guyton e Hall (1996).
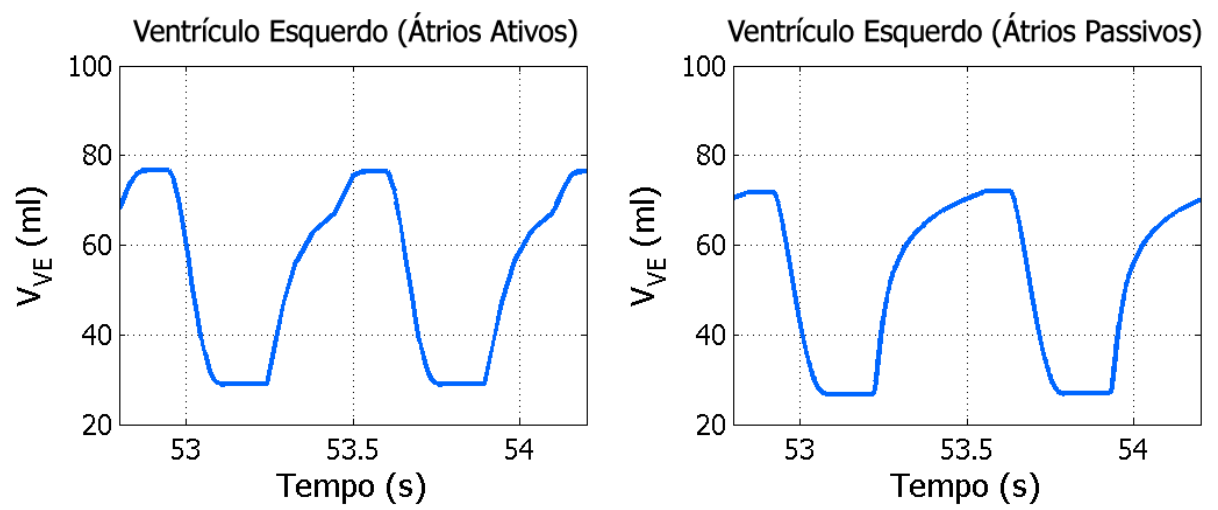

Figura 4-23 Volume no ventrículo esquerdo após (gráfico à esquerda) e antes (gráfico á direita) da inclusão da contratilidade atrial.

É possível afirmar que o formato da curva simulada está fisiologicamente adequado, apesar das diferenças de amplitude, tanto em valor entre extremos quanto em offset. Conforme mencionado, espera-se que uma sintonia de parâmetros mais aprofundada possa sanar estas variações, pois a representação fisiológica está bastante aderente.

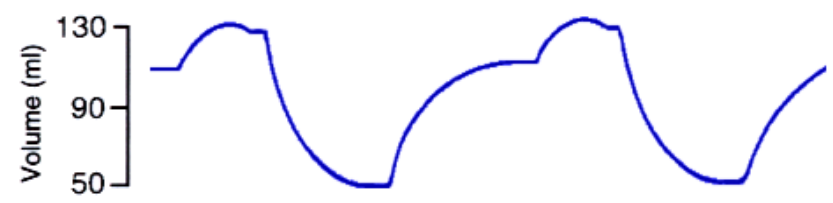

Figura 4-24 Curva de volume ventricular esquerdo adaptada de Guyton e Hall (1996). 
Finalmente, a Figura 4-25 mostra as curvas de volume no ventrículo direito. Novamente, notase o surgimento da onda de volume adicional devida à contração atrial, pouco antes da sístole ventricular.
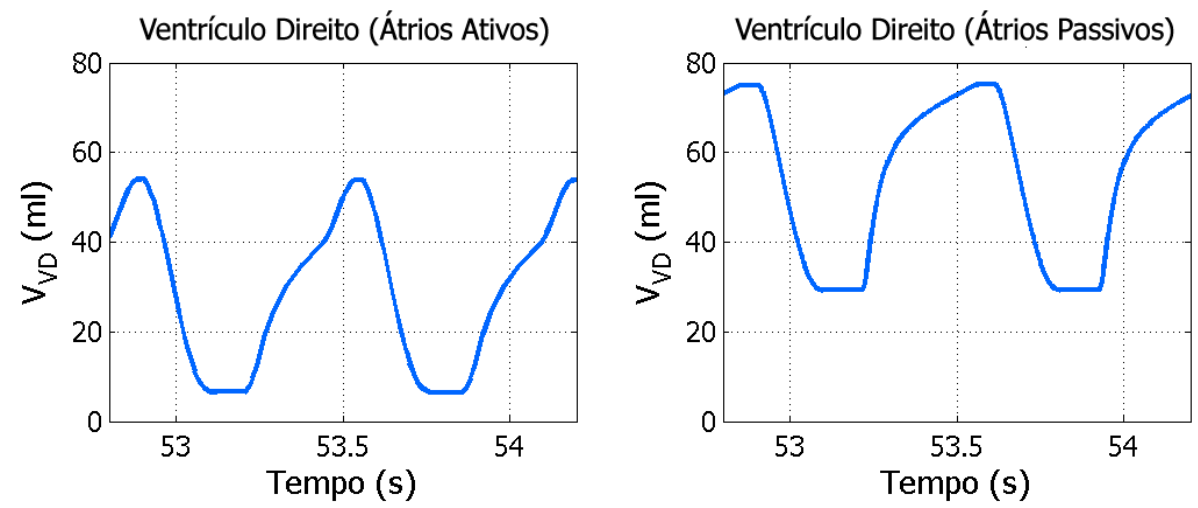

Figura 4-25 Curvas de volume no ventrículo direito, antes (gráfico da direita) e depois (gráfico da esquerda) da inclusão dos átrios ativos.

\subsubsection{Vazão Instantânea}

Adotando os parâmetros modificados mostrados no início deste capítulo, são apresentadas a seguir algumas curvas de vazão instantânea de saída dos ventrículos. Estes não são os valores de débito cardíaco, pois este é um parâmetro numérico obtido através da multiplicação do volume de deslocamento pela freqüência cardíaca. Como não há curvas de pacientes reais disponíveis, serão realizadas apenas algumas comparações com dados do trabalho de Ursino (1998). Mantém-se aqui a situação de regime, com o sistema em malha fechada.
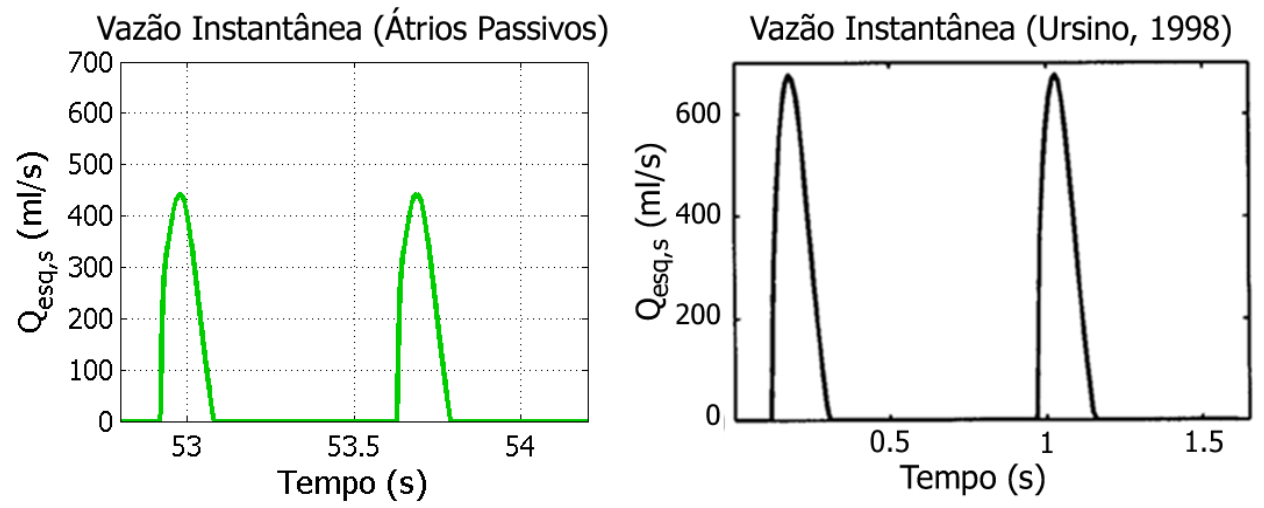

Figura 4-26 0 gráfico da esquerda mostra a curva de débito cardíaco resultante da simulação em Matlab do modelo com átrios passivos, utilizando os mesmos parâmetros sugeridos no trabalho de Ursino (1998). 0 gráfico da direita foi obtido diretamente do trabalho mencionado.

A Figura 4-26 acima mostra uma curva de débito cardíaco do ventrículo esquerdo resultante de uma simulação do modelo com átrios passivos. À direita desta figura foi colocado um gráfico extra- 
ído do trabalho de Ursino (1998), a efeito de comparação. Note que, apesar de aqui terem sido utilizados os mesmos parâmetros relatados no citado trabalho, a diferença de amplitude entre as duas curvas é bastante acentuada, mesmo que sua forma seja praticamente idêntica. Isso sugere que a simulação retratada na figura presente no artigo possa ser resultante de um conjunto de parâmetros diferente daquele listado por Ursino, e não necessariamente um erro de implementação.

Abaixo é mostrada uma segunda comparação de resultados de simulação com o mesmo gráfico da figura acima. Agora foi utilizado o modelo com átrios ativos, e nota-se novamente uma diferença considerável entre as duas curvas. Comparando os dois gráficos à esquerda da Figura 4-26 e da Figura 4-27, percebe-se também um aumento no débito cardíaco resultante da inclusão dos átrios ativos.
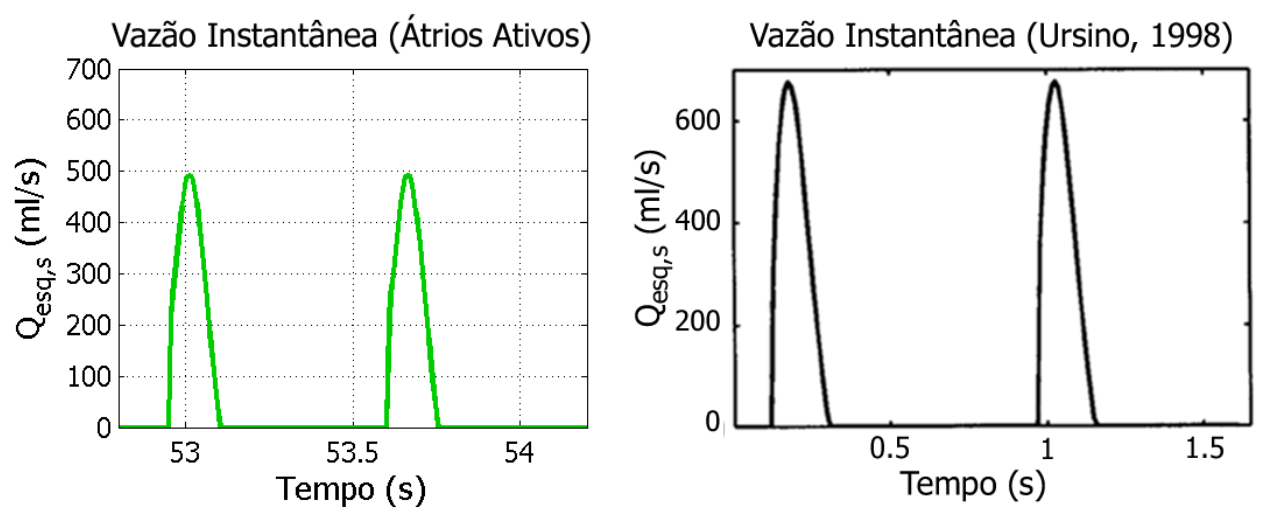

Figura 4-27 Simulação do débito cardíaco do ventrículo esquerdo do modelo com átrios ativos, em comparação com o mesmo gráfico extraído de Ursino (1998), mostrado na Figura 4-26 acima.

Apesar de não haver dados teóricos disponíveis para comparação, a Figura 4-28 abaixo mostra as curvas de débito cardíaco para o lado direito do coração.
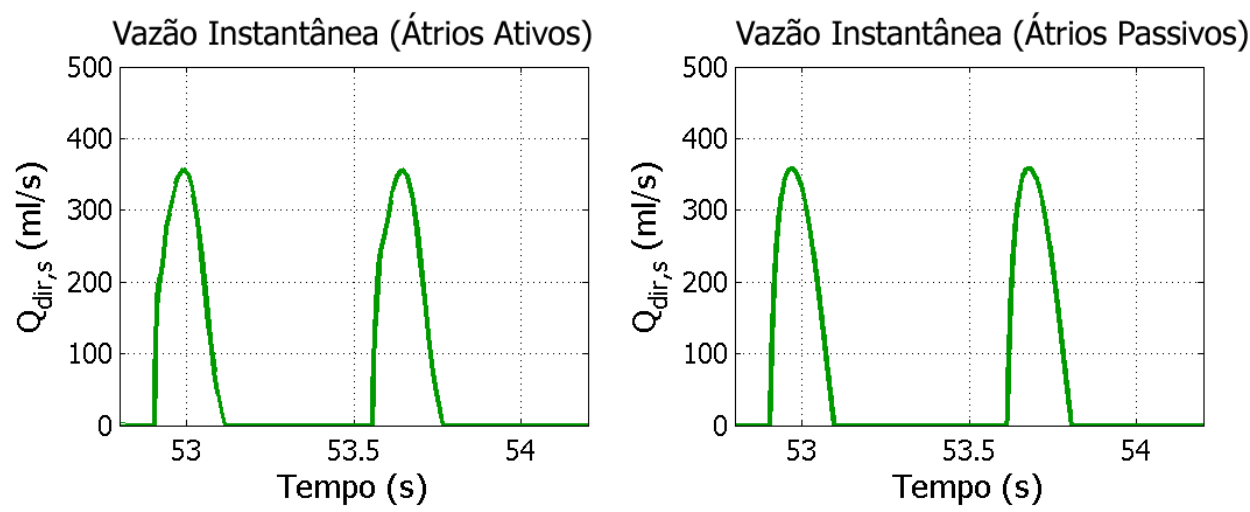

Figura 4-28 Comparação das curvas de débito cardíaco do lado direito do coração após (gráfico da esquerda) e antes (gráfico da direita) da inclusão da contratilidade dos átrios. 


\subsubsection{Ciclo Cardíaco}

O estudo da chamada bomba cardíaca é normalmente ilustrado pelo diagrama do ciclo cardíaco, composto por gráficos de pressão e volume do lado esquerdo do coração em função do tempo. A Figura 4-29 abaixo, extraída de Guyton e Hall (1996), exibe os eventos típicos que caracterizam o ciclo cardíaco (apesar de não serem de interesse para este trabalho, as curvas de eletrocardiograma e fonocardiograma foram deixadas como referência para o leitor).

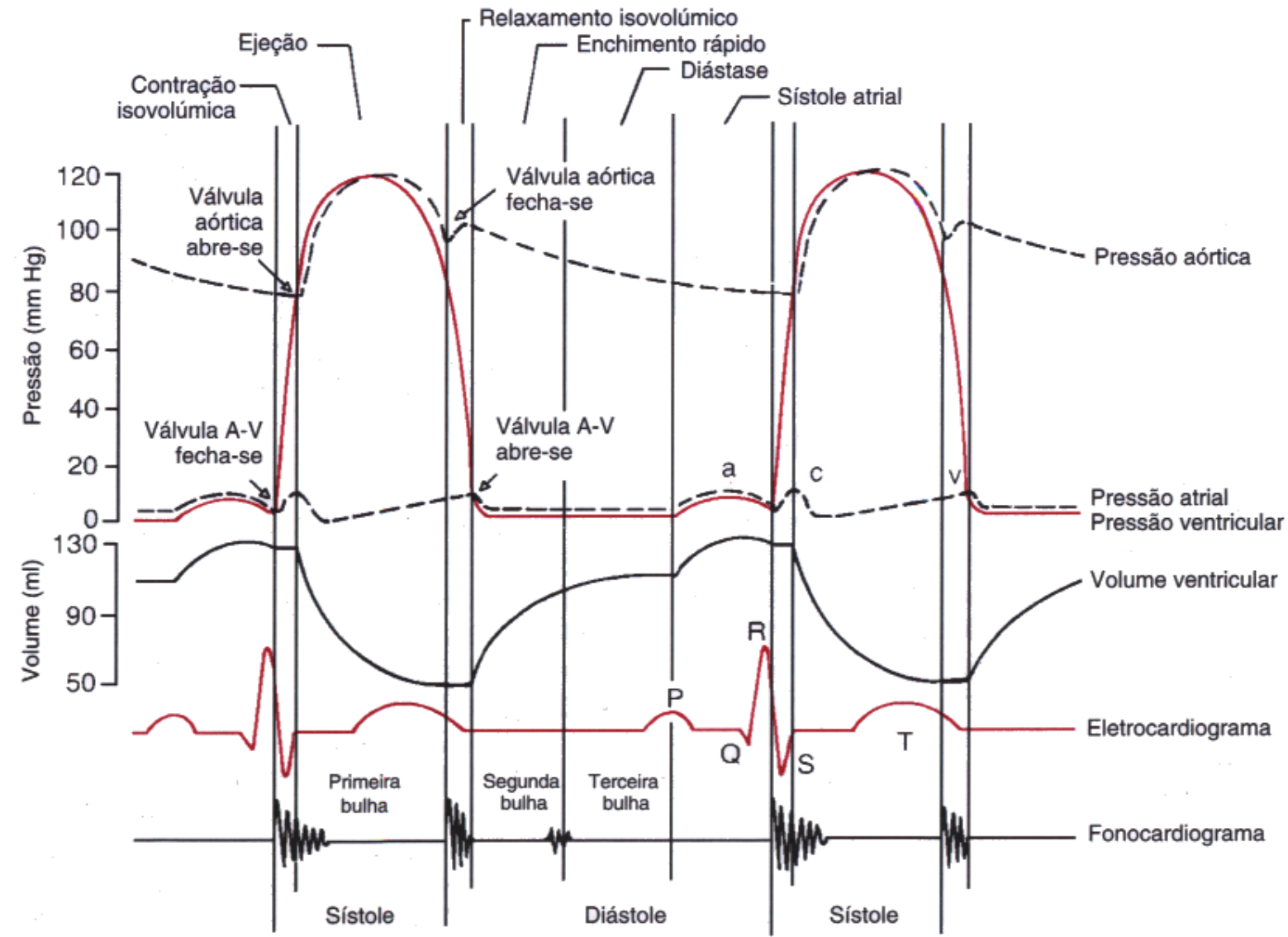

Figura 4-29 Ciclo cardíaco, mostrando as pressões aórtica, atrial e ventricular e o volume ventricular do lado esquerdo, bem como o eletro e o fonocardiograma (GUYTON e HALL, 1996). Assim como na Figura 4-15, estima-se o período cardíaco do gráfico entre 0.8 e $1 \mathrm{~s}$.

A simulação mostrada na Figura 4-30 foi feita com base nas mesmas curvas de pressão e volume contidas no diagrama do ciclo cardíaco, para comparação de algumas características do modelo, tendo sido adicionada apenas a curva de volume atrial. Conforme já comentado individualmente, percebe-se nos resultados que a simulação apresenta boa aderência para a pressão ventricular e para a pressão arterial, tanto na forma das ondas quanto nos valores numéricos. A pressão atrial, no entanto, ficou com amplitude muito baixa, mal aparecendo no gráfico comparativo. Em contrapartida, o 
volume ventricular passou a exibir a descontinuidade observada no terceiro terço da diástole do diagrama teórico, apesar de ainda não terem sido atingidos os valores por ela sugeridos. A curva de volume atrial acrescentada auxilia na compreensão geral do ciclo, para que sejam devidamente observados seus eventos característicos.
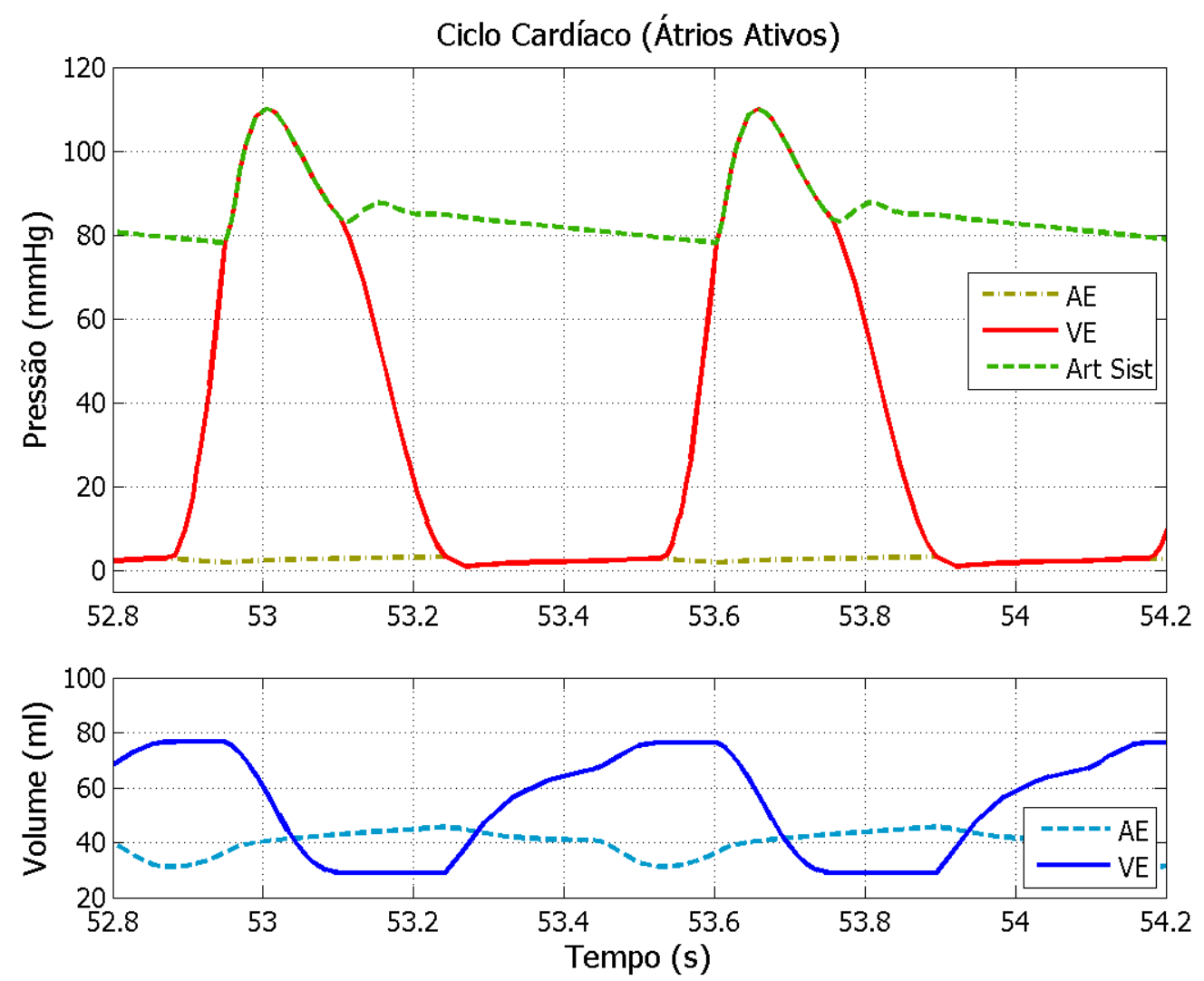

Figura 4-30 Simulação do ciclo cardíaco para o modelo com átrios ativos.

Para comparação, a Figura 4-31 abaixo mostra o mesmo ciclo cardíaco simulado no modelo com átrios passivos. Valem aqui todos os comentários feitos anteriormente na apresentação das curvas individuais de pressão e volume. Na pressão arterial, pode-se notar o efeito da inércia do sangue menos pronunciado, apesar de os valores estarem próximos. No caso da pressão atrial, devido às baixas amplitudes conseguidas nos átrios ativos, a aparência no ciclo é praticamente a mesma, ou seja, quase não se vê oscilação. É nas curvas de volume que as diferenças se tornam mais evidentes. A elevação do volume ventricular devida à sístole atrial está completamente ausente no caso do modelo com átrios passivos, por razões óbvias (se não há sístole atrial, é impossível que aconteça a respectiva elevação de volume). A curva de volume atrial fica bastante característica de um comporta- 
mento passivo, de enchimento e esvaziamento elástico do compartimento em função da passagem do sangue.
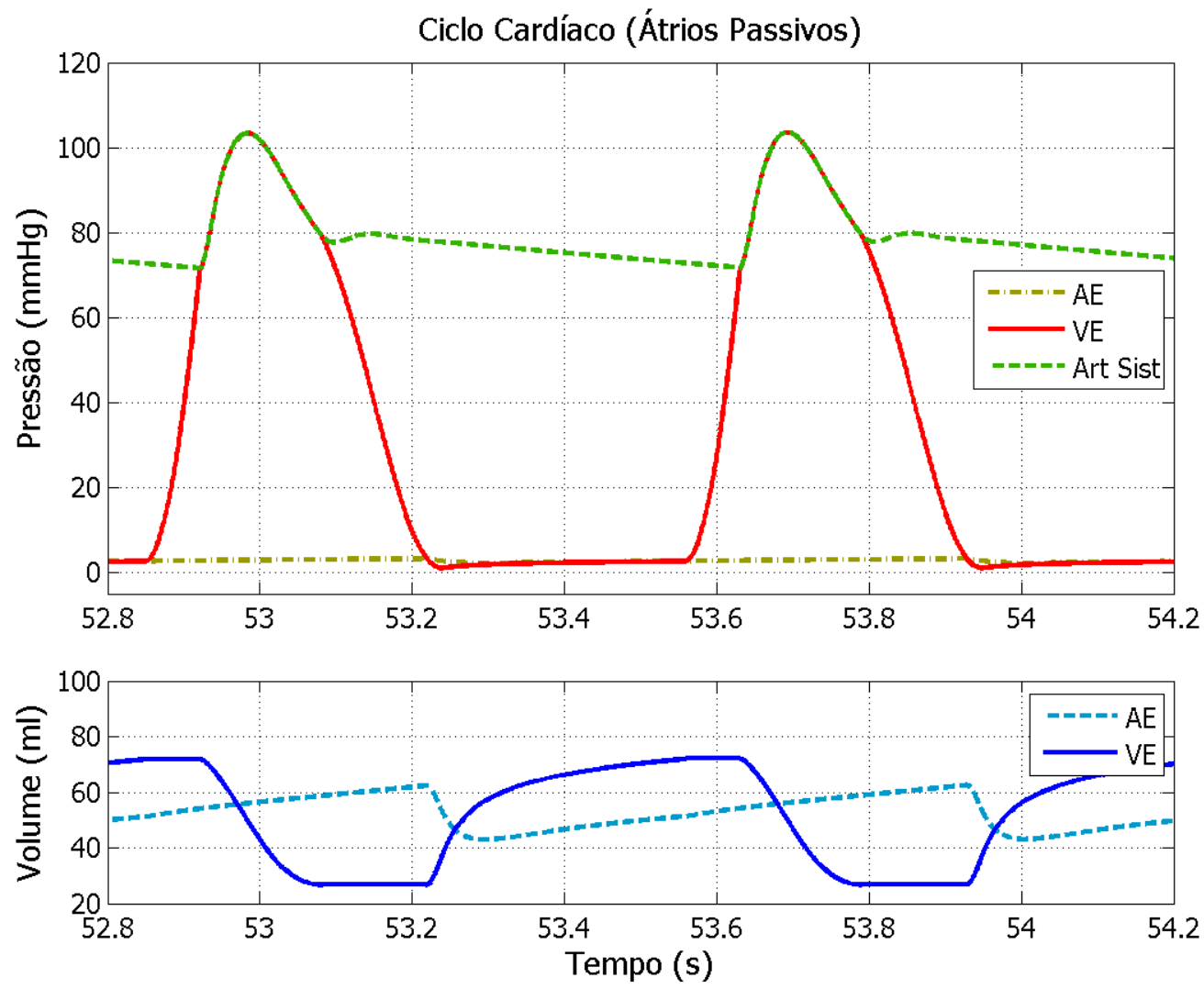

Figura 4-31 Simulação do ciclo cardíaco para o modelo com átrios passivos.

\subsubsection{Alças Pressão-Volume}

As alças (loops) pressão-volume, mostradas anteriormente no Capítulo 3 durante a explicação dos parâmetros ventriculares e na determinação dos parâmetros atriais, são muito utilizadas na explicação e estudo das fases do ciclo cardíaco e também na caracterização das alterações mecânicas provocadas por certos eventos e patologias. Serão apresentados aqui alguns resultados referentes às alças obtidas para os ventrículos e também para os átrios, para ambos os lados do coração, sempre que possível em comparação com dados da literatura.

A Figura 4-32 abaixo, adaptada de Antoni (1996a), conceitualiza de uma forma mais visual a determinação de uma alça pressão volume (a figura retrata o ventrículo esquerdo). O autor utiliza a analogia de um espelho para associar a transformação de eixo do volume. Desta forma, fica mais 
clara a relação entre as fases do ciclo cardíaco e as laterais da forma da alça. Evidentemente, esta mesma analogia é válida para os demais compartimentos do coração.

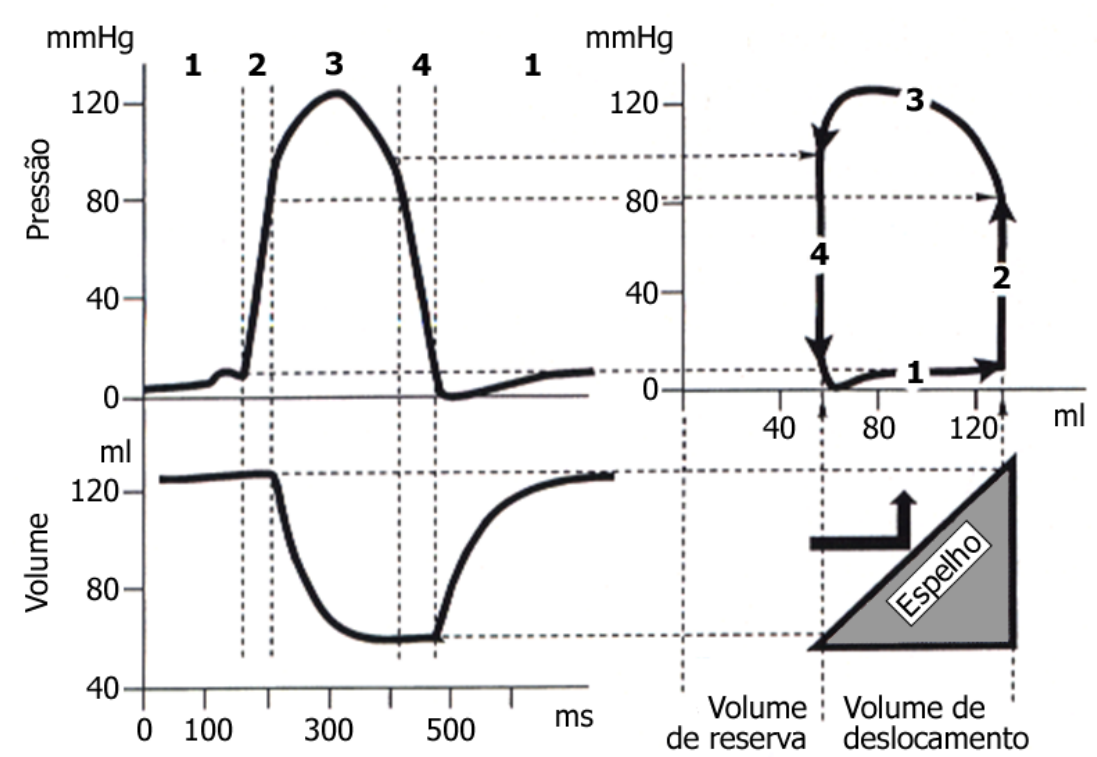

Figura 4-32 Determinação da alça pressão-volume do ventrículo esquerdo (adaptado de Antoni, 1996a).

\section{Ventrículos}

A Figura 4-33 mostra um exemplo de alça pressão-volume para o ventrículo esquerdo correspondente a valores normais de pressão e volume (RHOADES; TANNER, 2003). O painel superior direito da Figura 4-32 também mostra valores de normalidade para o mesmo ventrículo.

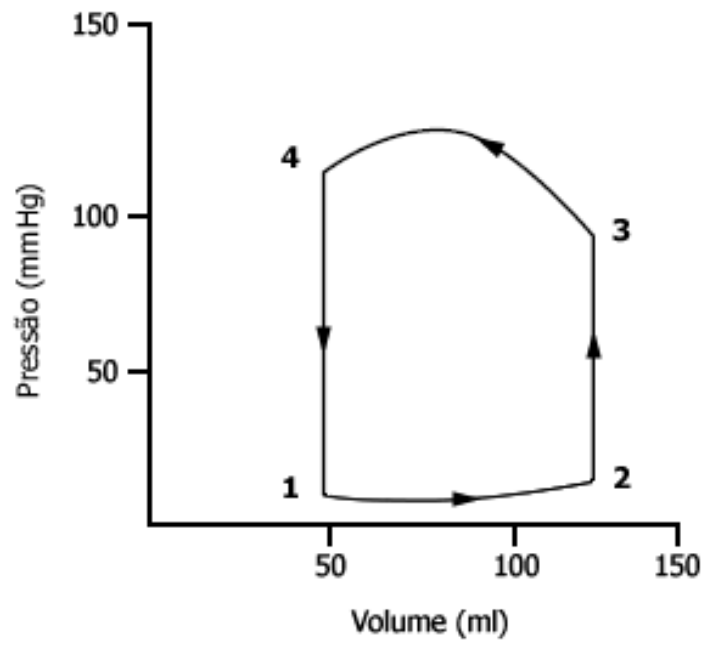

Figura 4-33 Alça pressão-volume do ventrículo esquerdo (RHOADES e TANNER, 2003).

A Figura 4-34 mostra a mesma curva, agora como resultado da simulação numérica do modelo com átrios ativos. Qualitativamente, a curva está bastante adequada, com os trechos de contração 
(2-3) e relaxamento (4-1) isovolúmicos, de enchimento (1-2), e de ejeção (3-4) devidamente caracterizados. Nota-se, no entanto, que a alça encontra-se deslocada cerca de $20 \mathrm{ml}$ para a esquerda em relação aos dados de Rhoades e Tanner (2003), mostrados na Figura 4-33, e de Antoni (1996a), mostrados na Figura 4-32. Além disso, o volume de deslocamento (stroke volume), que consiste na distância entre as duas fases isovolumétricas (segmentos $2-3$ e 4-1), é cerca de $20 \mathrm{ml}$ menor que os valores teóricos. Observando-se as considerações feitas para as curvas de volume, esse deslocamento já era esperado, e deve-se aos parâmetros utilizados nas simulações.

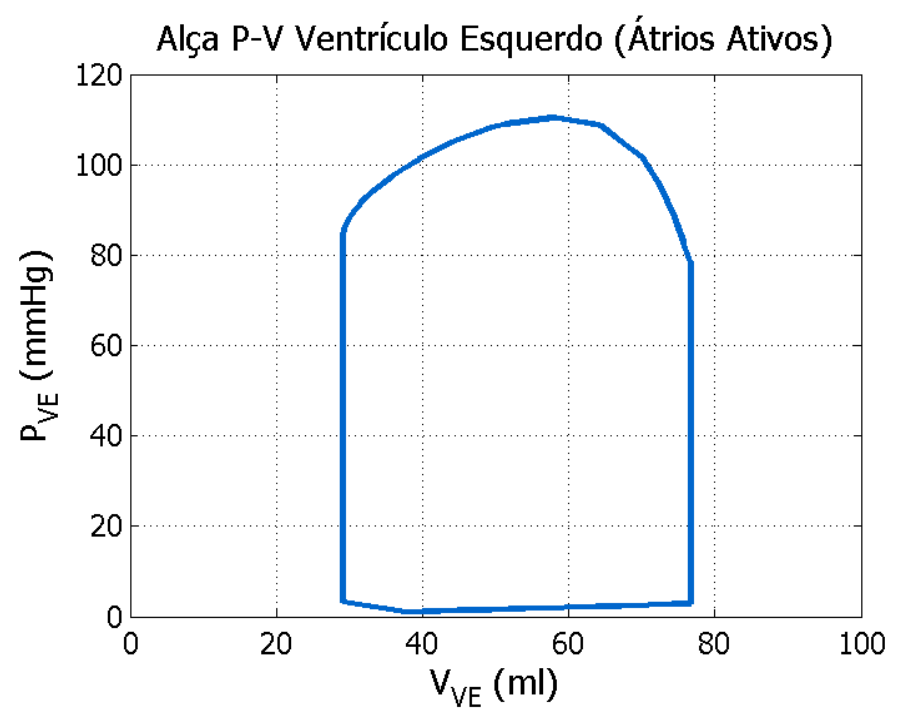

Figura 4-34 Alça pressão-volume calculada para o ventrículo esquerdo (modelo com átrios ativos).

A alça correspondente ao modelo com átrios passivos é mostrada na Figura 4-35. Para este compartimento, a adição dos átrios ativos provocou alterações mínimas de comportamento.

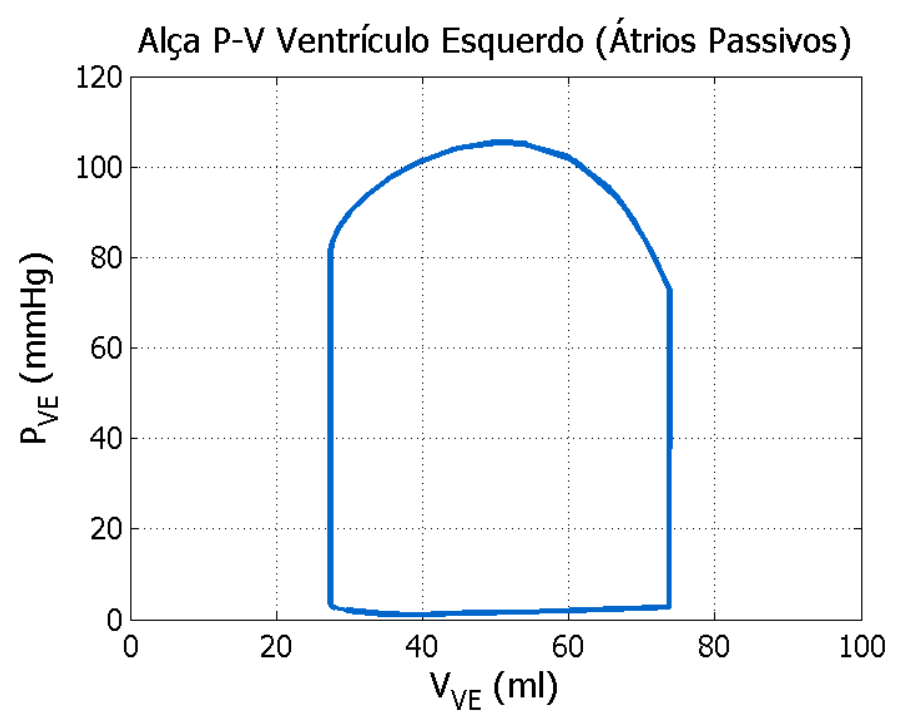

Figura 4-35 Alça pressão-volume calculada para o ventrículo esquerdo (modelo com átrios passivos). 
Para a alça pressão-volume do ventrículo direito poderão ser feitas apenas comparações entre os modelos com átrios ativos e passivos, pois não foram encontradas curvas para este compartimento na literatura. A Figura 4-36 mostra a alça P-V do ventrículo direito para o modelo com átrios ativos. Em comparação com a Figura 4-37, correspondente aos átrios passivos, nota-se uma enorme diferença, tanto em forma quanto em valores numéricos. Considerando que o comportamento dos dois lados do coração deveria ser semelhante, apenas com proporções diferentes, a primeira alça parece ser mais adequada, devido à sua maior proximidade de forma com a alça do ventrículo esquerdo. Infelizmente, nada além do que foi mencionado na apresentação das curvas de pressão e volume pode ser acrescentado com relação aos valores, pois não há uma alça teórica para comparação.

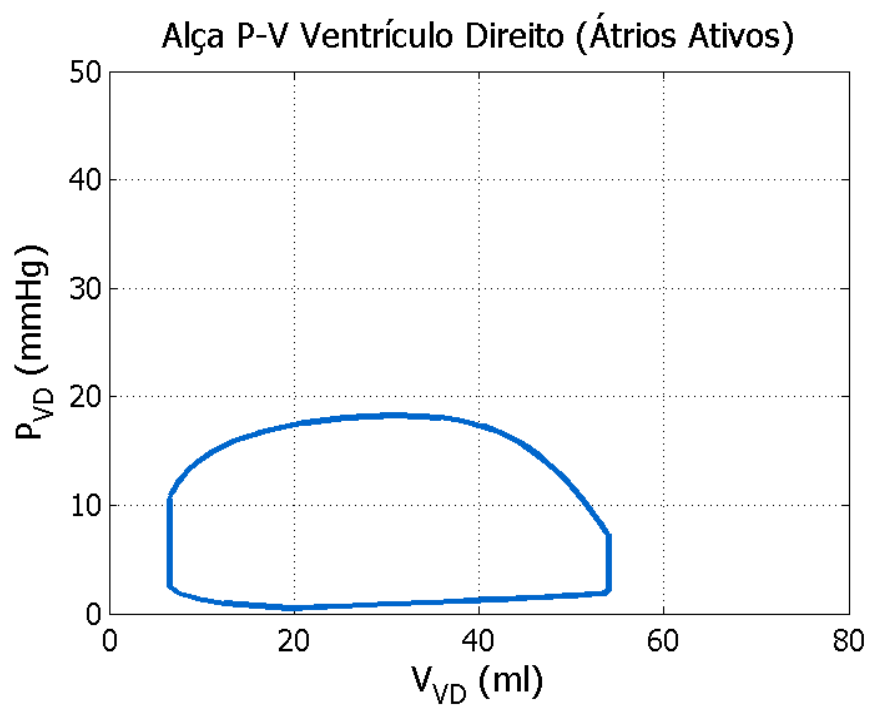

Figura 4-36 Alça pressão volume calculada para o ventrículo direito (modelo com átrios ativos).

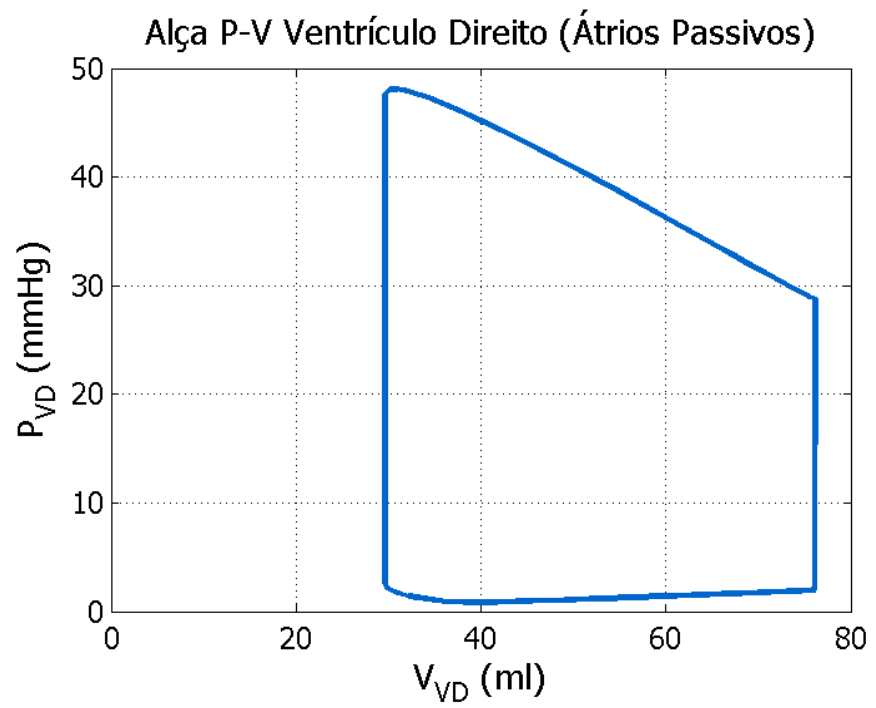

Figura 4-37 Alça pressão volume calculada para o ventrículo direito (modelo com átrios passivos). 


\section{Átrios}

As alças pressão volume tiveram papel fundamental na modelagem dos ventrículos (no trabaIho de Ursino, 1998) e também dos átrios ativos, conforme mostrado no Capítulo 3. Conforme então mencionado, as curvas disponíveis na literatura para as alças atriais são experimentais, e apresentam elevada variabilidade numérica em decorrência das dificuldades de medição. Mesmo assim, sabe-se que o ciclo sempre descreve a forma de um algarismo oito deitado, como visto na Figura 4-38 para o átrio esquerdo.

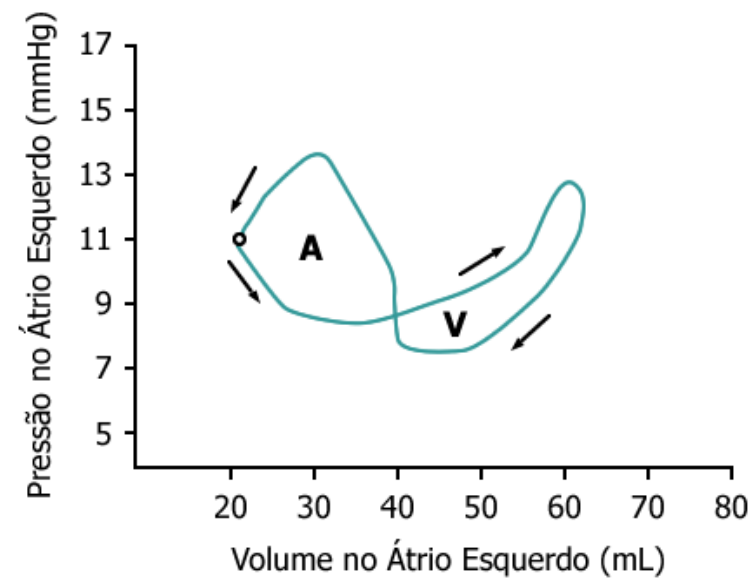

(a)

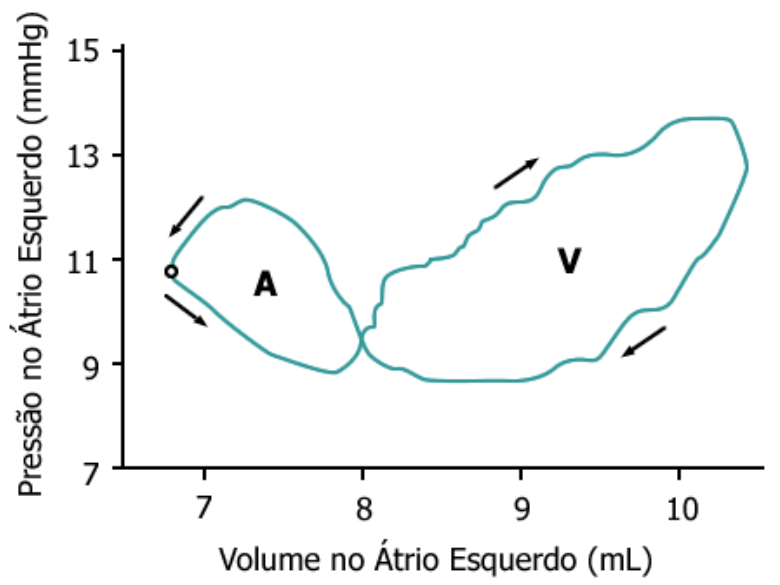

(b)

Figura 4-38 Alças pressão-volume experimentais determinadas para o átrio esquerdo, adaptadas de (a) Matsuda et al. (1983), e de (b) Pagel et al. (2003).

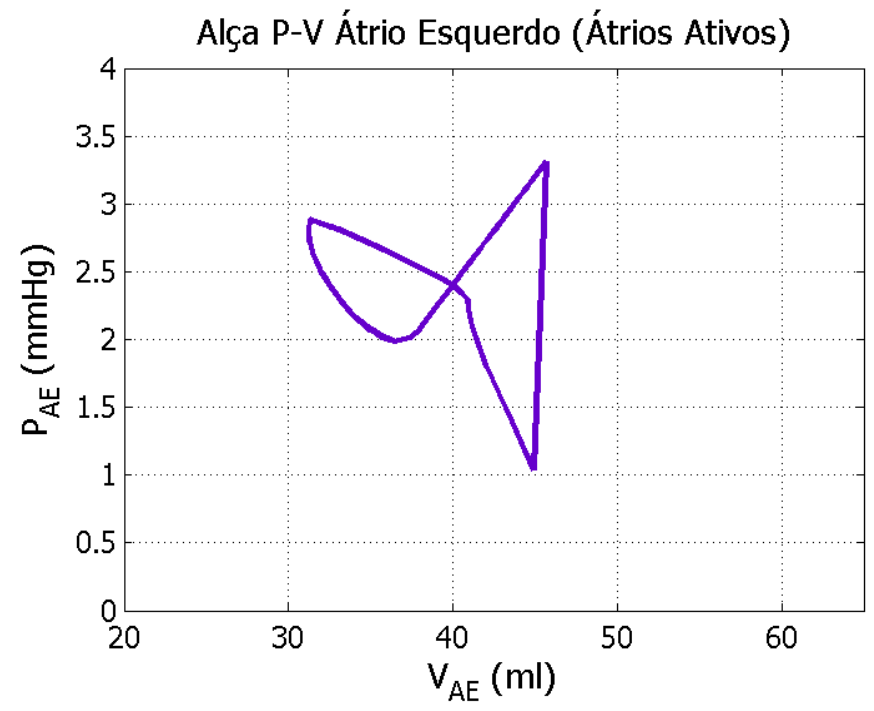

Figura 4-39 Alça pressão-volume calculada para o átrio esquerdo (modelo com átrios ativos).

A Figura 4-39 acima mostra a alça pressão-volume do átrio esquerdo resultante da simulação do modelo com átrios ativos. Observe que o formato de oito foi de fato descrito, apesar de ligeira- 
mente deformado. Como as alças de referência também não constituem unanimidade, pode-se considerar satisfatória a forma simulada. Os valores de pressão, como anteriormente comentado, estão bastante inferiores aos esperados, mas o volume está adequado. A Figura 4-40 abaixo mostra a forma descrita pelo modelo com átrios passivos. Como não há atividade atrial, não se forma uma figura fechada.

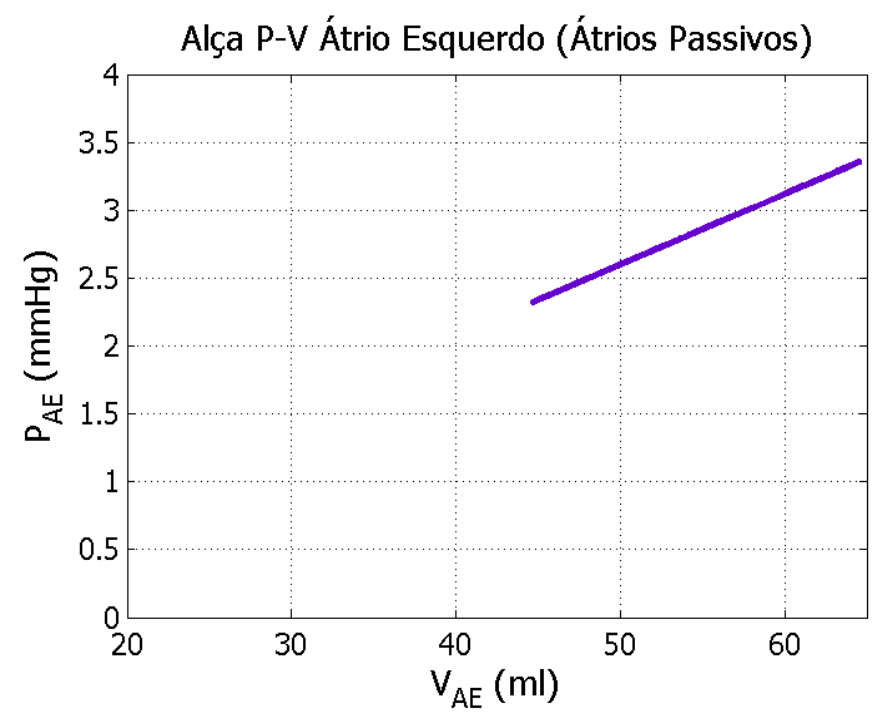

Figura 4-40 Relação pressão-volume para o átrio esquerdo (modelo com átrios passivos).

Para o átrio direito também foram encontrados na literatura apenas resultados experimentais, repetindo-se as considerações sobre a variabilidade das medições. A Figura 4-41 abaixo mostra resultados de dois trabalhos sobre o comportamento mecânico do átrio direito.

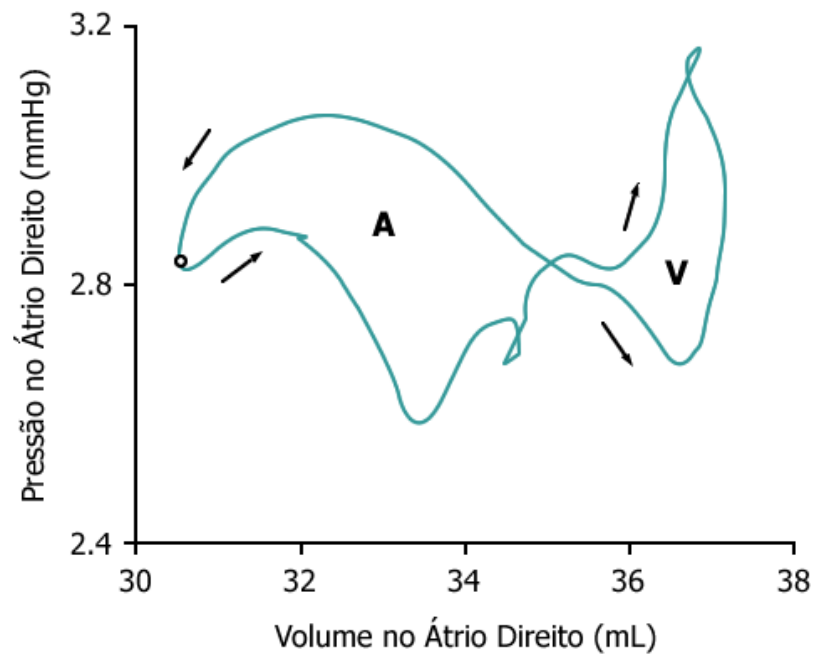

(a)

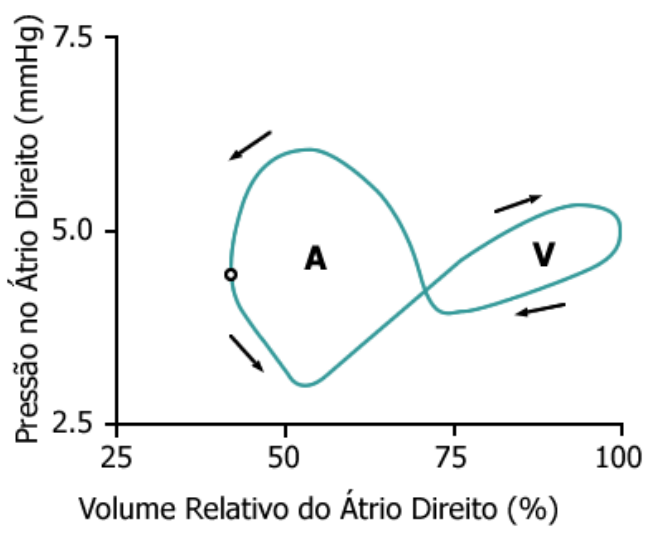

(b)

Figura 4-41 Alças pressão-volume experimentais determinadas para o átrio direito, adaptadas de (a) Maniar et al. (2003), e de (b) Miller et al. (1986). 
A Figura 4-42 abaixo mostra a alça P-V para o átrio direito resultante da simulação do modelo com átrios ativos. Novamente, é possível identificar a forma de oito, porém desta vez bastante deformada. Durante os testes de ajustes de parâmetros a forma da figura variou bastante, sendo que a configuração utilizada nos demais resultados apresentados até aqui foi a mais satisfatória. Conforme comentado anteriormente, os valores de pressão ficaram muito abaixo dos valores esperados, mas a faixa de variação do volume está dentro dos valores mostrados na Figura 4-41.

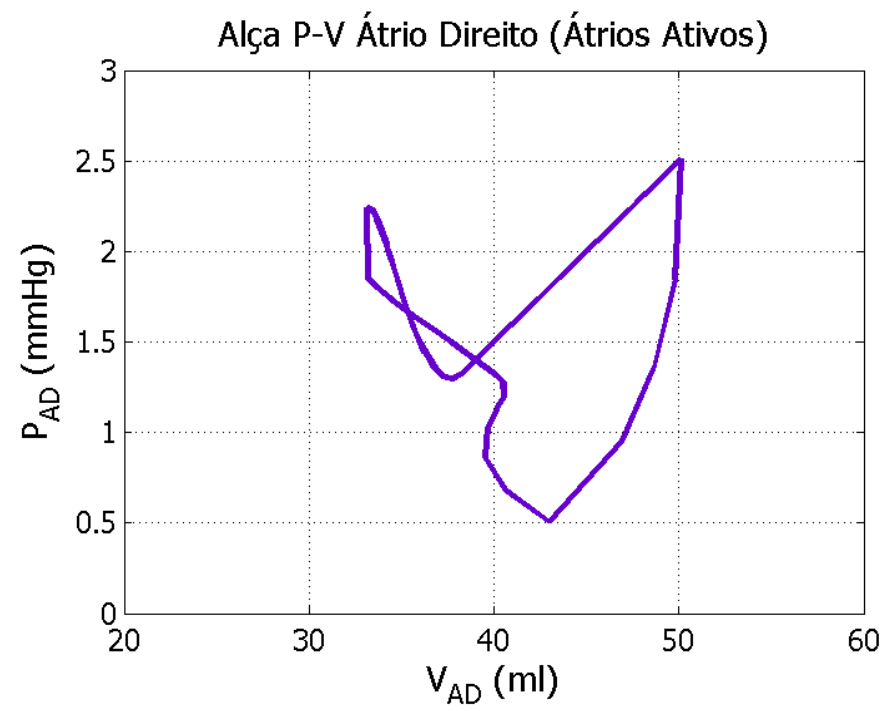

Figura 4-42 Alça pressão-volume calculada para o átrio direito (modelo com átrios ativos).

A alça do átrio direito resultante do modelo com átrios passivos é mostrada na Figura 4-43 abaixo. Novamente, a relação é linear, devido à ausência de atividade contrátil no átrio.

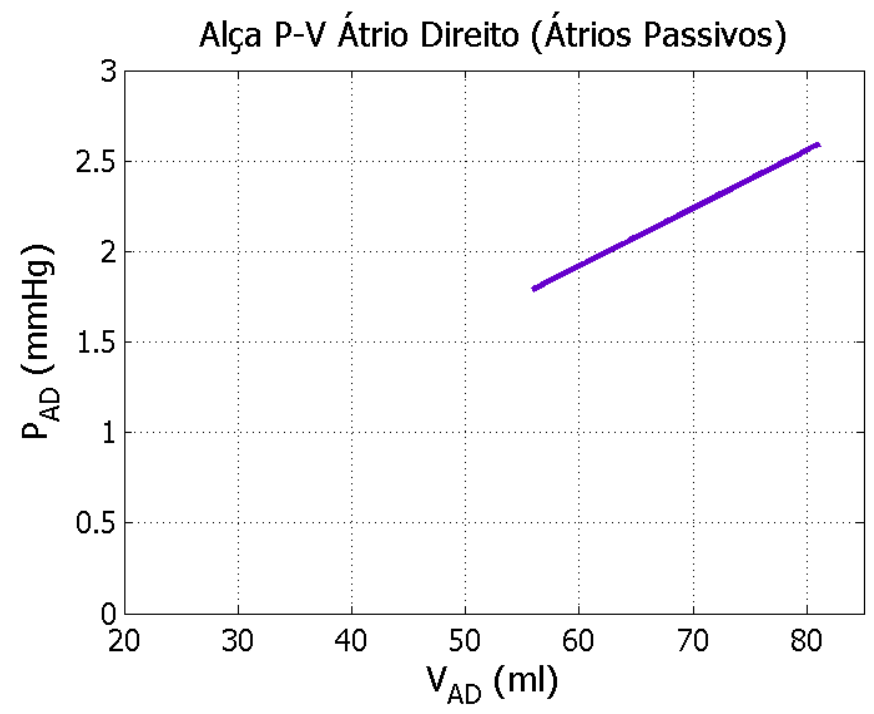

Figura 4-43 Relação pressão-volume para o átrio direito (modelo com átrios passivos). 


\subsubsection{Resposta do Mecanismo Barorreflexo}

Para demonstrar o funcionamento do sistema de controle pelo mecanismo barorreflexo, principalmente com relação a características de desempenho, foram feitas algumas simulações. Nas figuras a seguir foi utilizado um período de 100 segundos, mantendo o mesmo solver ode45 (DormandPrince) com intervalo de tempo variável. A pressão no ventrículo esquerdo foi escolhida como parâmetro de comparação, sendo mostrada na Figura 4-44 abaixo em uma simulação partindo de condições iniciais não nulas (extraídas da situação final de uma simulação anterior) até o atingimento da situação de regime.

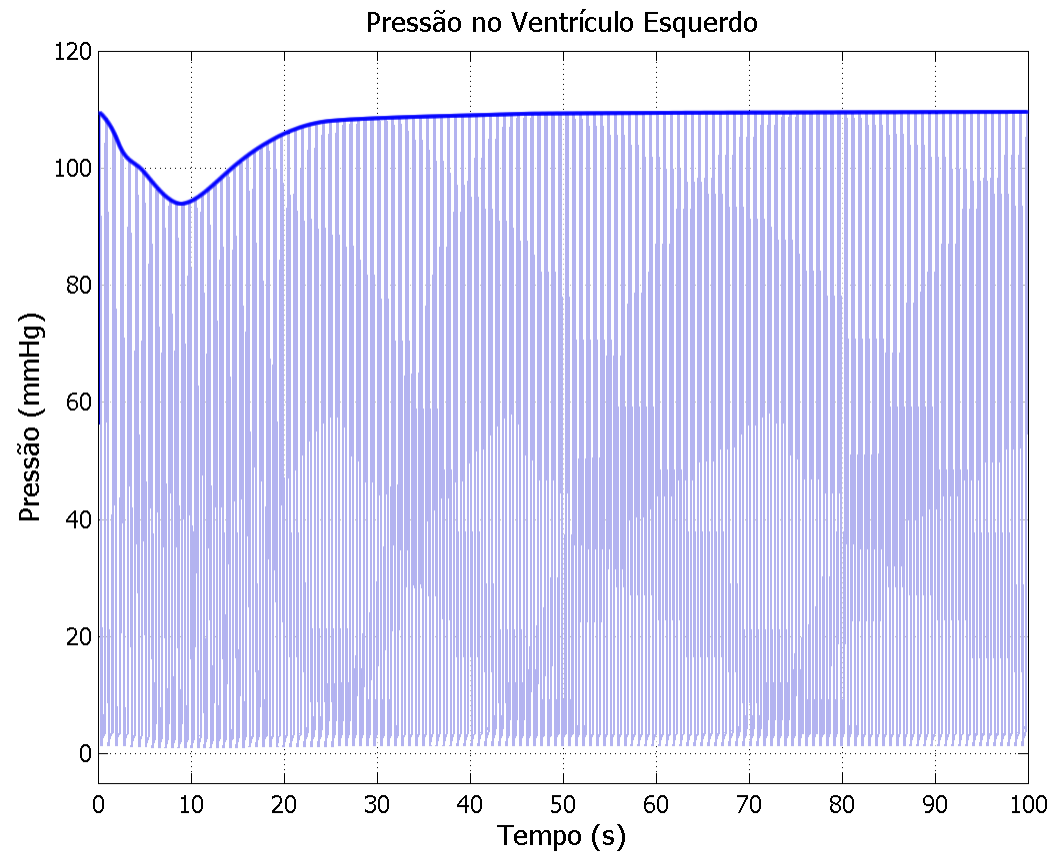

Figura 4-44 Pressão no ventrículo esquerdo. A curva mais clara corresponde à pressão pulsátil, efetiva no ventrículo, e a linha mais escura representa apenas o envelope da curva, para maior clareza.

Apesar de este gráfico não mostrar muito sobre a adequação do modelo em relação à fisiologia (mesmo que os valores extremos estejam corretamente dimensionados), pode-se perceber que ele leva pouco mais de 20 segundos para atingir a condição de regime, com o sistema estabilizado.

Foi realizada também uma simulação de perda de sangue, para verificar a atuação do sistema de controle em resposta a uma entrada degrau aplicada no volume sangüíneo total. O degrau foi inserido após 50 segundos de simulação, com o sistema em regime, consistindo em uma queda de $500 \mathrm{ml}$ (quase $10 \%$ ) no volume de sangue contido no sistema (que era de $5300 \mathrm{ml}$ ) - o que corresponde a 
uma hemorragia acentuada. 0 tempo de acomodação foi de cerca de 20 segundos, conforme observado na Figura 4-45.

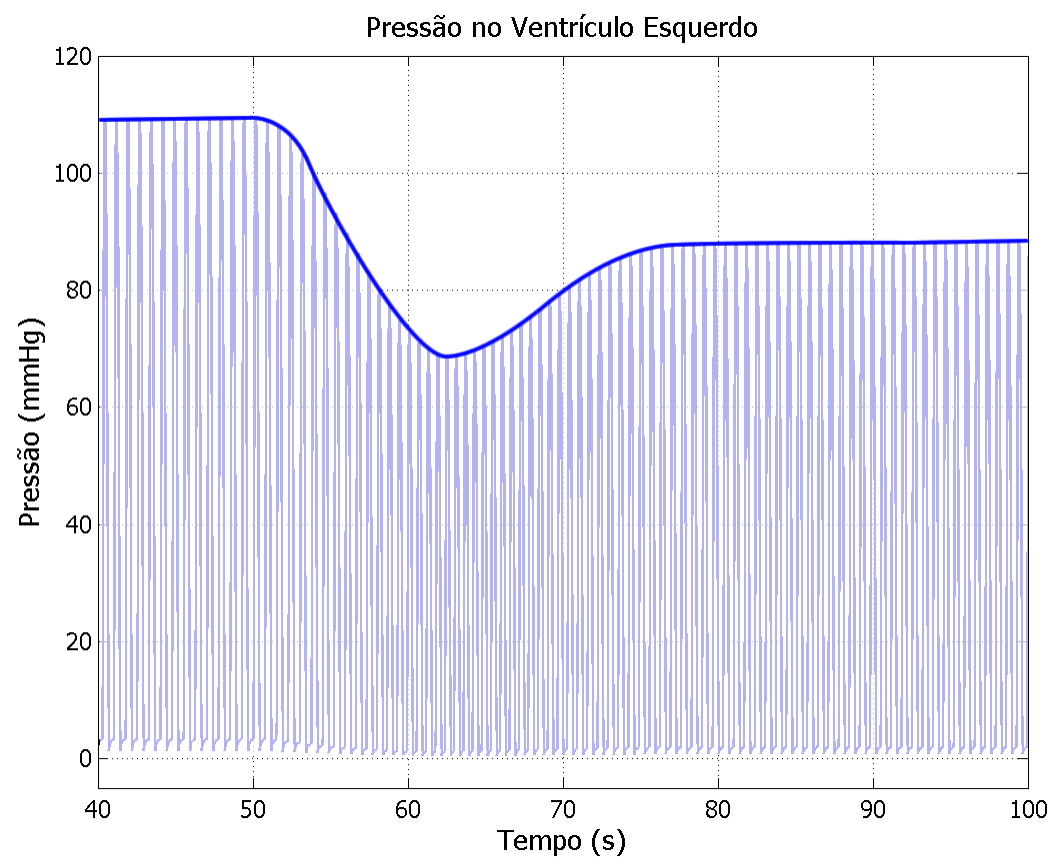

Figura 4-45 Simulação de perda de um volume de sangue de $500 \mathrm{ml}$ ( $10 \%$ do volume total considerado). Novamente, a curva mais clara corresponde à pressão pulsátil e a linha mais escura representa o envelope da curva. 


\section{Desenvolvimento do Simulador Didático}

\subsection{Proposta}

A análise da literatura atual deixou evidente a falta de trabalhos que explorem a simulação didática do sistema cardiovascular humano. Existem muitos trabalhos ressaltando os benefícios da simulação no ensino de fisiologia, mas quase todos são focados nos simuladores em escala natural, baseados em bonecos. Apesar de essenciais para o treinamento clínico, estes equipamentos são bastante caros, dependentes de um ambiente hospitalar, e voltados para estudantes em nível de residência e profissionais com experiência. Trabalhos como o de Michael (2001) apontam que a simulação é uma ferramenta extremamente eficiente na formação dos conceitos básicos de fisiologia, em especial nos primeiros anos de estudo. Os experimentos de Michael ressaltam que mesmo o uso de simuladores extremamente simples já consegue promover nos alunos uma maior capacidade investigativa, estimulando-os a compreender as causas e conseqüências dos sistemas fisiológicos e a abandonar a tradicional memorização excessiva de conceitos prontos.

Um trabalho anterior (LONARDONI; IWATA, 2002), voltado para a simulação do sistema respiratório (ver Figura 5-1), indicou grande receptividade por parte dos usuários a aplicativos acessíveis e fáceis de utilizar. Acessíveis, pois estes programas devem ser facilmente distribuídos e capazes de rodar em computadores com baixa capacidade de processamento. Fáceis de utilizar, pois o usuário não pode se sentir intimidado pelo programa, ou incapaz de encontrar as funções e parâmetros desejados.

No caso do sistema cardiovascular, foram encontrados na literatura poucos trabalhos que se aproximam destes requisitos. Os trabalhos citados por Michael não estavam disponíveis - com exceção do projeto CIRCSIM-Tutor, que ainda está em fase de desenvolvimento. O simulador apresentado por Rothe e Gersting (2002), baseado em um modelo simples, de cinco compartimentos (ver Item 2.3.3 acima), possui recursos interessantes, como os tutoriais e sua distribuição (em CDs), mas peca na usabilidade. A interface é pouco amigável, com muitos valores numéricos simultâneos, e seus cálculos são baseados em ciclos estáticos, não em tempo real. 


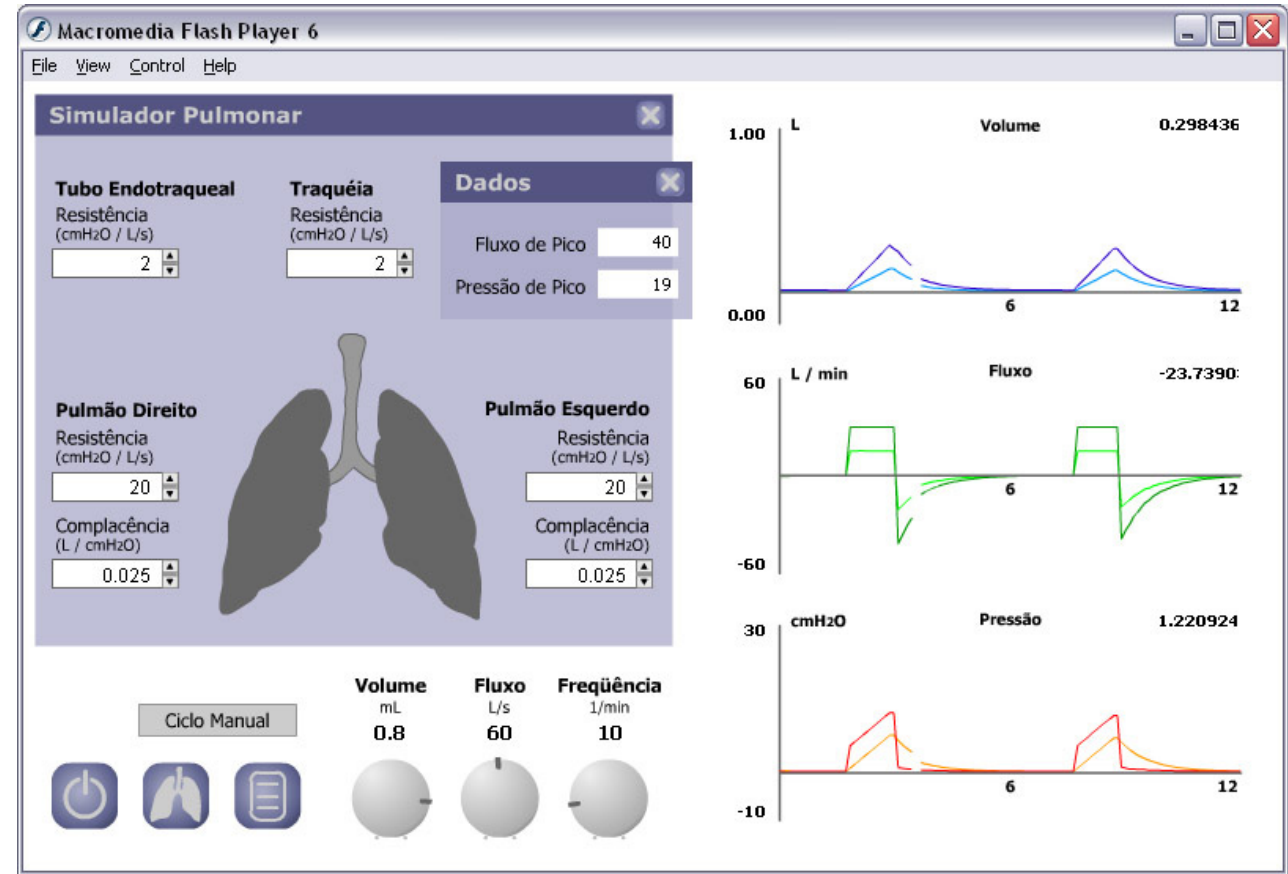

Figura 5-1 Simulador do sistema respiratório desenvolvido em trabalho anterior (LONARDONI e IWATA, 2002).

Motivada pelo (inesperado) sucesso do trabalho de 2002 e pelas pesquisas didáticas de Michael (2001), esta dissertação propõe o desenvolvimento de um simulador do sistema cardiovascular humano que cumpra os requisitos mencionados. A idéia é que o aplicativo represente o sistema de forma realista, além de ser cognitivamente ergonômico e de fácil distribuição. Estes tópicos serão abordados a seguir.

\subsection{Seleção do Modelo}

Para que o sistema cardiovascular seja representado de forma realista, deve ser utilizado um modelo capaz de conferir às simulações resultados qualitativos que se aproximem dos valores esperados. Além disso, com a finalidade de oferecer um alto nível de interatividade (para melhor compreensão de mecanismos de causa e efeito), espera-se que o simulador seja capaz de reagir em tempo real às intervenções do usuário. O primeiro requisito poderia ser conseguido com o uso de roteiros e valores gravados, atingindo os valores mais acurados possíveis (afinal, eles teriam sido extraídos de casos reais). No entanto, haveria enorme perda de interatividade, com alterações limitadas a valores discretos, contidos nos conjuntos de dados armazenados. 
Desta forma, a melhor saída seria a utilização de um modelo matemático rodando por trás da interface do aplicativo, fornecendo em tempo real as saídas correspondentes às requisições do usuário. O modelo de Ursino (1998) acrescido da atividade atrial contrátil, apresentado no Capítulo 3, foi selecionado por apresentar respostas bastante fiéis (qualitativa e quantitativamente) à literatura. 0 modelo é complexo, incluindo uma quantidade razoável de compartimentos e uma representação do mecanismo barorreflexo, suficientes para contabilizar os efeitos fisiológicos mais relevantes. Ainda assim, as simulações numéricas realizadas se mostraram rápidas, ao menos no ambiente do Simulink.

\subsection{Discretização e Algoritmo de Integração}

Para o desenvolvimento do simulador, foi necessária a discretização do modelo, para que as etapas de cálculo fossem executadas passo a passo. Por simplicidade, optou-se pelo uso de um integrador trapezoidal com intervalo de tempo fixo, utilizando-se nas equações a diferença entre o valor da variável no passo atual e no passo anterior. As equações escritas desta forma foram colocadas em um arquivo $\mathrm{M}$ do Matlab, e simuladas para a determinação do intervalo de tempo mínimo necessário. Testes indicaram que o sistema se comportava da mesma forma que o modelo em Simulink (rodando algoritmo de passo variável e integrador ode45) a partir de um intervalo menor que $0.001 \mathrm{~s}$.

\subsection{Linguagens de Programação}

No desenvolvimento anterior (LONARDONI; IWATA, 2002) foi utilizado como ambiente de programação o Macromedia Flash MX (com linguagem Actionscript 1.0). Esta escolha foi feita devido a dois aspectos principais: a usabilidade e a portabilidade. $O$ primeiro se refere, na verdade, à facilidade em se desenvolver aplicações interativas, que ofereçam variados recursos multimídia. O segundo se refere à quantidade de plataformas capazes de rodar o plugin do Flash sem que haja a necessidade de recompilação do código.

No entanto, no final deste processo de desenvolvimento anterior, foi detectado um problema de desempenho. O Flash, e mais especificamente a linguagem Actionscript em sua implementação (retroativamente denominada) 1.0, não é uma linguagem de programação otimizada para executar cálculos. Como o desenvolvimento do simulador cardiovascular envolve cálculos significativamente 
mais complexos que os anteriores, surgiu o receio de que o Flash não seria capaz de, sozinho, prover o desempenho esperado. Por este motivo, foram estudadas algumas alternativas de integração de linguagens, além da análise da chamada versão 2.0 da linguagem Actionscript, incorporada ao Flash MX 2004 e Flash 8.

\subsubsection{Actionscript 2.0}

Antes de testar qualquer alternativa mais trabalhosa, optou-se pela verificação do desempenho da linguagem Actionscript 2.0, supostamente mais rápida ${ }^{1}$ que a versão 1.0. Apesar dos problemas de desempenho experimentados anteriormente (percebido apenas em máquinas mais antigas), o Flash continuava apresentando o menor tempo de desenvolvimento, justificando ao menos alguns testes antes de uma decisão mais definitiva.

Foi inicialmente desenvolvida uma classe para desenho de gráficos no interior de uma janela de pop-up, e incluídos alguns controles básicos para interação com o modelo, conforme mostrado na Figura 5-2. As equações escritas no arquivo $M$ mencionado acima foram traduzidas para a linguagem Actionscript, e colocadas no aplicativo em duas etapas - inicialmente sem e depois com o sistema de controle por barorreflexo. A primeira etapa indicava um desempenho satisfatório, apesar de já demandar uma estratégia de compensação do tempo de execução do programa, capaz de fazer o tempo de desenho passar mais lentamente que o tempo de cálculo, de modo a fornecer tempo hábil para o processamento de um passo completo. Antes da inclusão do sistema de controle, o fator de tempo utilizado nesta estratégia era igual a 10, ou seja, o tempo do simulador era dez vezes mais lento que o tempo real.

Apesar de provocar certo desconforto por ter-se que aguardar muito tempo pelo traçado dos gráficos, passou-se à etapa de inclusão das equações de controle. O resultado foi a necessidade de

\footnotetext{
${ }^{1}$ A linguagem Actionscript original está formalmente presente no Macromedia Flash desde sua versão 5, recebendo maiores avanços na versão MX (também conhecida como 6). Esta foi a versão utilizada no desenvolvimento anterior, e que apresentava problemas de desempenho em certas máquinas. Desde o lançamento da versão MX 2004 (também conhecida como 7), o Macromedia Flash passou a incorporar a nova implementação da linguagem Actionscript, denominada de 2.0 (a partir deste momento, a primeira implementação da linguagem Actionscript foi retroativamente denominada Actionscript 1.0, por motivo de diferenciação), com sintaxe de orientação a objetos baseada no padrão ECMAScript 4. Isso implica na incorporação formal de uma sintaxe e metodologia de programação orientada a objetos à linguagem Actionscript, proporcionando maior desempenho.
} 
um aumento do fator de tempo de 10 para 100, e ainda com alguns travamentos durante a alteração de parâmetros. Tamanho desconforto para o usuário inviabilizaria o uso prático do aplicativo, o que motivou a busca por alternativas mais robustas.

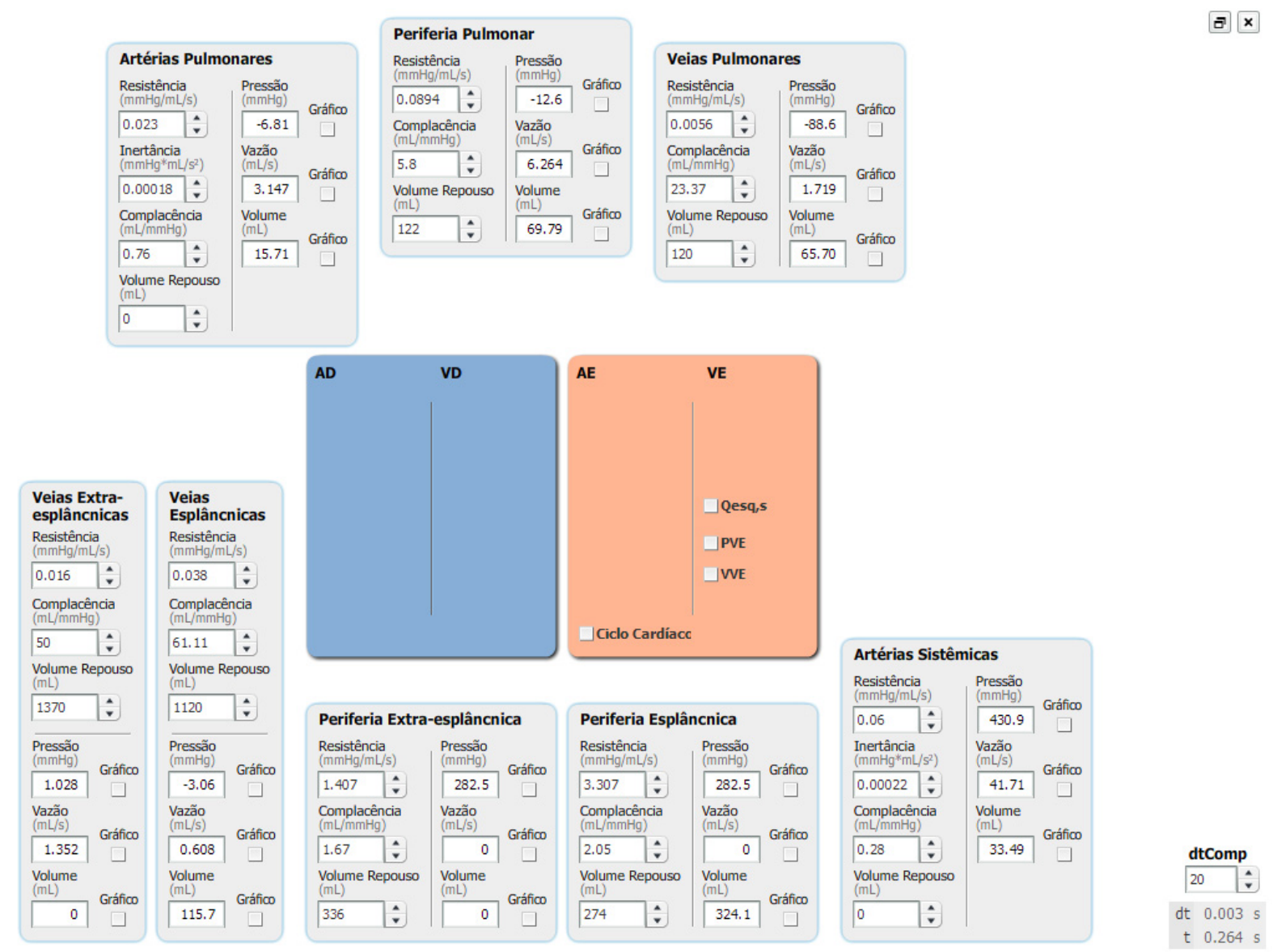

Figura 5-2 Simulador preliminar desenvolvido apenas em Flash e Actionscript 2.0.

\subsubsection{Integração com Matlab/Simulink}

A primeira possibilidade de integração de linguagens considerada foi entre a linguagem C++ (com a qual existe maior familiaridade) e a API do Matlab, para facilitar e otimizar a realização de cálculos. Antes mesmo de testar a viabilidade técnica desta alternativa, a mesma foi desfavorecida pela descoberta de que seria necessário que o usuário final do programa possuísse o Matlab instalado em sua máquina para poder funcionar (contrariando uma das premissas estabelecidas para o simulador).

No mesmo sentido, as tentativas de integração entre Flash e Matlab/Simulink foram desestimuladas por também exigirem o Matlab no computador do usuário final. A idéia seria utilizar como 
base o aplicativo GUIDE (Graphical User Interface Development Environment) do Matlab, com a inclusão de componentes ActiveX para o uso de elementos criados em Flash.

\subsubsection{Integração entre Flash e C++}

Com a finalidade de reduzir a carga computacional exigida no simulador final foram estudadas as possibilidades de integração entre o Macromedia Flash e a linguagem C++. A comunicação entre os dois ambientes permite que se use código em $\mathrm{C}++$ com elementos de interface em Flash. A integração é feita através de um componente ActiveX disponível para o Microsoft Windows (Shockwave Flash component) colocado em uma janela criada em C++. A comunicação entre estes dois ambientes é realizada através de um único comando, capaz de passar apenas um parâmetro por chamada. Desta forma, como o fluxo de informações necessário para a plotagem dos gráficos em tempo real é muito grande, poderia haver certo comprometimento do desempenho do programa.

\subsubsection{Integração entre $\mathrm{C}++\mathrm{e} \mathrm{Qt}$}

Ainda utilizando o $\mathrm{C}++$ como base, foi considerado o uso da biblioteca gráfica Qt para o desenho dos gráficos e demais elementos de interface. Esta biblioteca, de código aberto, é independente de plataforma, permitindo que um mesmo código possa ser compilado em qualquer um dos principais sistemas operacionais da atualidade (Windows, Linux, MacOS). A linguagem utilizada nativamente pela biblioteca é $\mathrm{C}++$, resolvendo de imediato os problemas de integração entre ambientes.

Alguns testes foram realizados, inclusive com a implementação do modelo completo, com todas as equações da dinâmica e do controle, e o ambiente se mostrou bastante eficiente. No entanto, a biblioteca apresenta apenas elementos de interface comuns, limitando o resultado desejado.

\subsubsection{Integração entre Flash e C\#}

Diferentemente do caso anterior, a comunicação entre Flash e C\# pode ser feita através de uma API especial da Macromedia (Flash External API), desenvolvida originalmente para uso com Javascript. Esta API, apesar de utilizar o mesmo controle ActiveX mencionado acima, permite a passagem de uma maior quantidade de parâmetros simultâneos entre os aplicativos, além de possibilitar a chamada de funções de um ambiente para o outro. 
Alguns testes foram executados, resultando em um ambiente básico para o simulador capaz de efetuar todos os cálculos envolvidos, incluindo as equações de controle, além de plotar gráficos em Flash em tempo real. Além disso, com o Flash é possível desenvolver todos os elementos de interface desejados para o simulador final. Pela versatilidade, esta foi a ferramenta selecionada para a implementação do simulador.

\subsection{Interface e Arquitetura Funcional}

O desenvolvimento da interface de simulação procurou priorizar os conceitos de Ergonomia Cognitiva, conceito utilizado no desenvolvimento de interfaces homem-máquina. Através destes conceitos, procura-se otimizar as interfaces para que sua utilização seja a mais intuitiva possível e para que qualquer usuário seja capaz de operá-la com um mínimo de conhecimento específico ou treinamentos prévios.

A interface do programa foi desenvolvida com base em dois modos de operação, um básico (mostrado na Figura 5-3), com uma interface mais simplificada e recursos mais limitados, e um avançado (mostrado na Figura 5-5), com visualização simultânea de diversas variáveis e possibilidade de controle de todos os parâmetros. Em ambos os modos, podem-se visualizar gráficos de qualquer variável sendo traçados em tempo real, da mesma forma que ocorre em um monitor de pacientes hospitalar. Nas figuras abaixo, que mostram a tela principal do programa em seus dois modos de operação, pode-se notar as duas abas responsáveis pela alternância entre os modos, posicionadas na parte superior da janela, logo abaixo da barra de título.

Também é possível observar nas figuras abaixo o nome dado ao simulador, CVBSim (com subtítulo Simulador do Sistema Cardiovascular controlado pelo Mecanismo Barorreflexo) - CV representa cardiovascular e $B$ representa o Barorreflexo. Este nome foi selecionado por ser representativo tanto em português quanto em inglês, pois se planeja uma eventual tradução do aplicativo para este último idioma. 


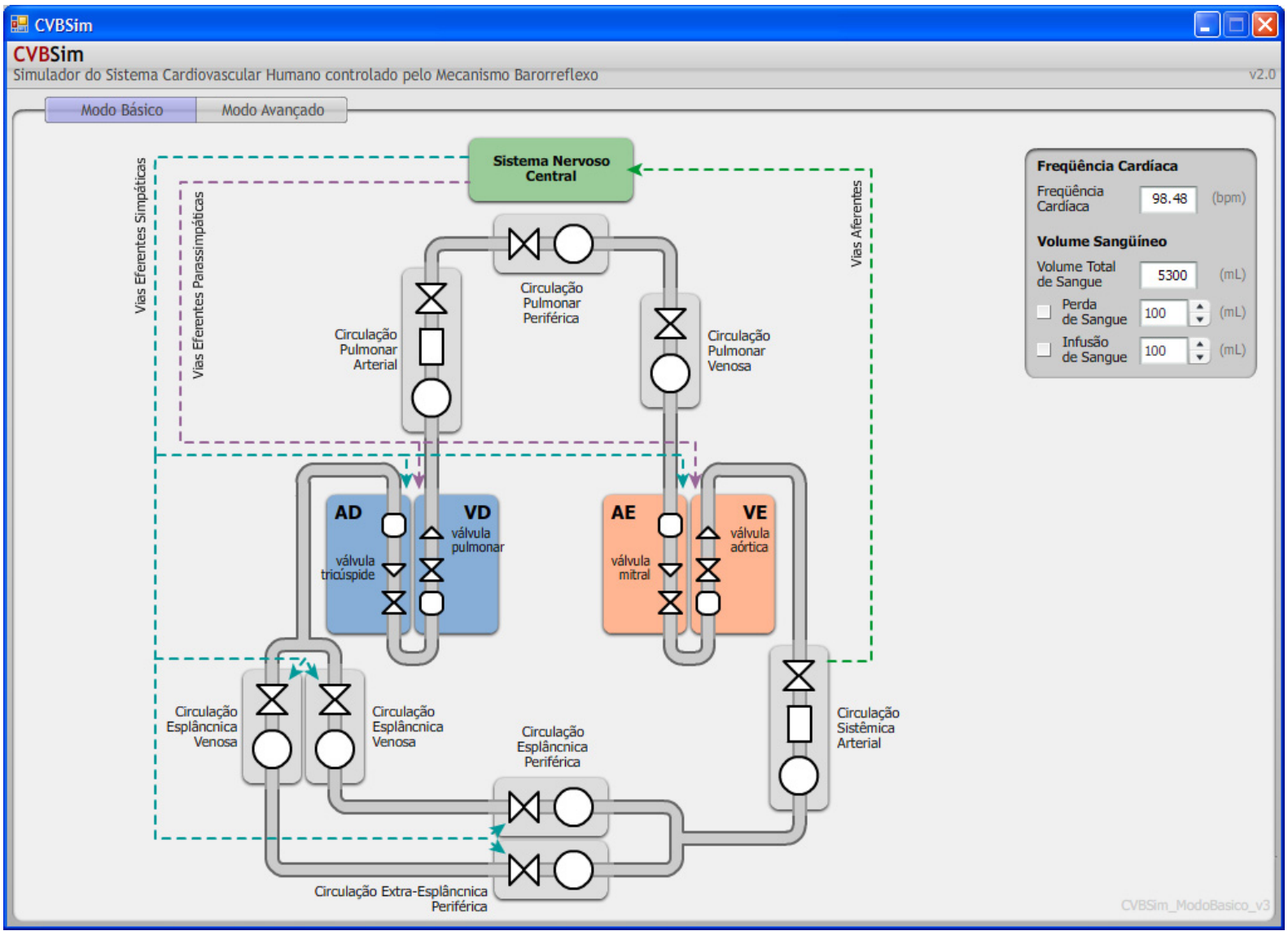

Figura 5-3 Interface do simulador no modo básico de operação.

A visualização de gráficos no modo básico é feita através do mouse, clicando-se diretamente sobre os compartimentos da figura - uma janela de exemplo pode ser vista na Figura 5-4. Os únicos monitores permanentes neste modo são de pressão arterial média e de débito cardíaco, mais comuns em monitores de paciente. $O$ controle de hemorragia permite a simulação de perda de sangue no paciente simulado, para que se verifique a atuação do controle em função da queda de pressão sangüínea. Um degrau de volume igual ao valor ajustado na caixa numérica é aplicado ao volume total de sangue assim que a caixa de seleção é habilitada.

No modo avançado, são visualizados permanentemente os valores instantâneos de pressão, fluxo e volume de todos os compartimentos. A visualização dos respectivos gráficos é feita através das caixas de seleção ao lado das caixas de visualização dos valores numéricos. Ao desabilitar as caixas de seleção, os gráficos correspondentes são ocultados. Os parâmetros são alterados através do botão Parâmetros, disponível para todos os compartimentos. Através deste botão, é acessada a respectiva janela de configuração de parâmetros, visualizada na Figura 5-6 a seguir para as artérias sistêmicas, 
para periferia pulmonar e para o coração direito. O controle de hemorragia continua disponível, com função idêntica à do modo básico.

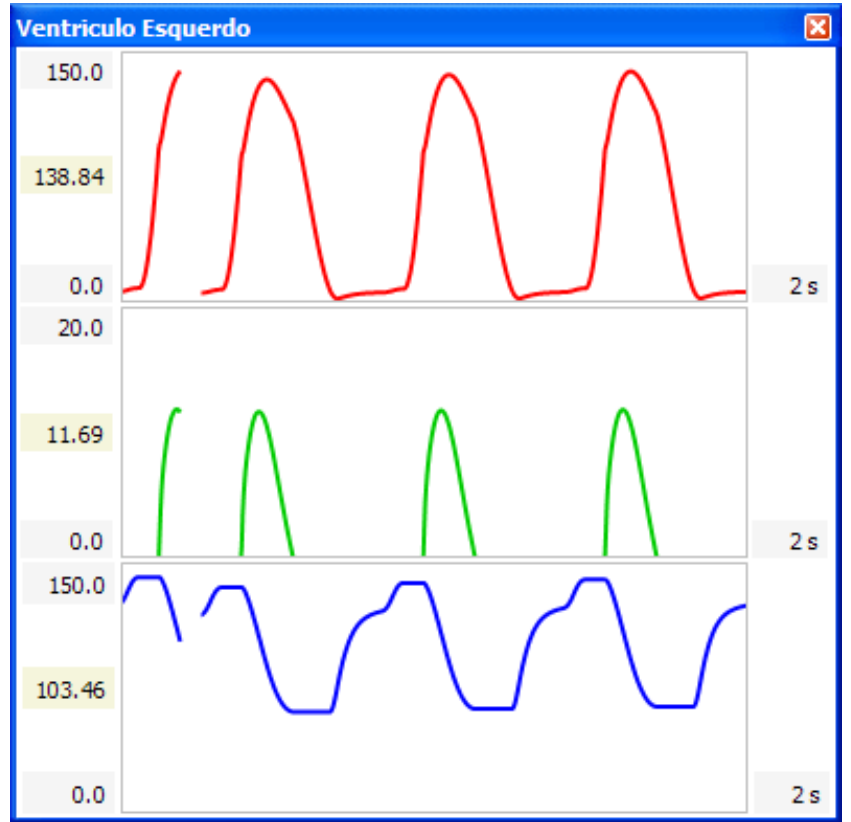

Figura 5-4 Janela de plotagem do modo básico, onde pode-se observar as três curvas (pressão, vazão e volume) simultaneamente.

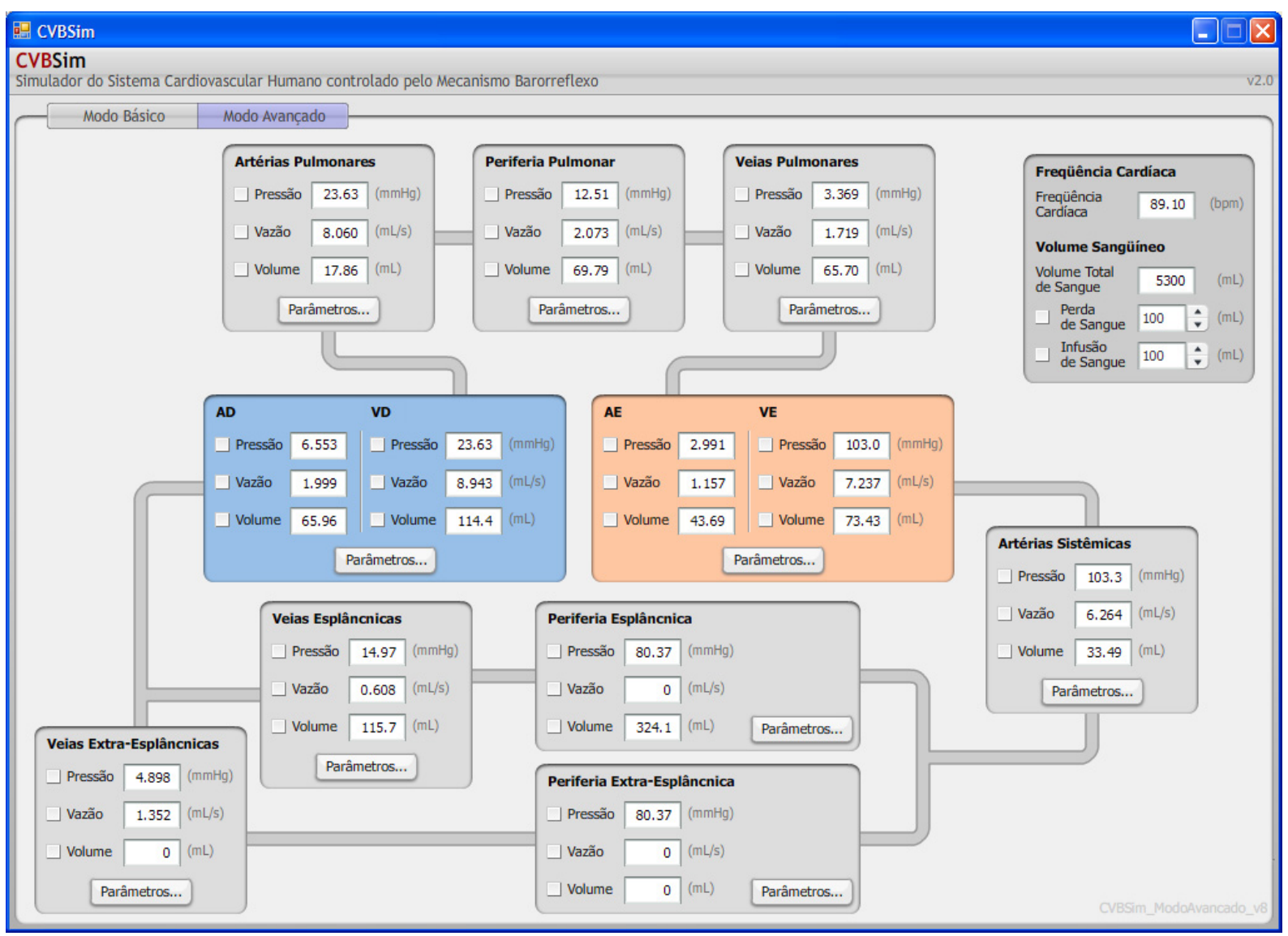

Figura 5-5 Interface do simulador no modo avançado de operação. 
Observe que, na Figura 5-5, a cor do fundo do controle de hemorragia é diferenciada em relação aos monitores, assim como acontece com os corações direito e esquerdo. O motivo destas diferenciações é facilitar a associação cognitiva entre as cores e as funções - perceba que as cores dos dois lados do coração são as mesmas utilizadas no modo básico. Apesar de no momento existir apenas o controle de hemorragia como função especial, a intenção é permitir a inclusão de novas funções, fazendo este tipo de associação mais importante no sentido de manter a clareza da interface.

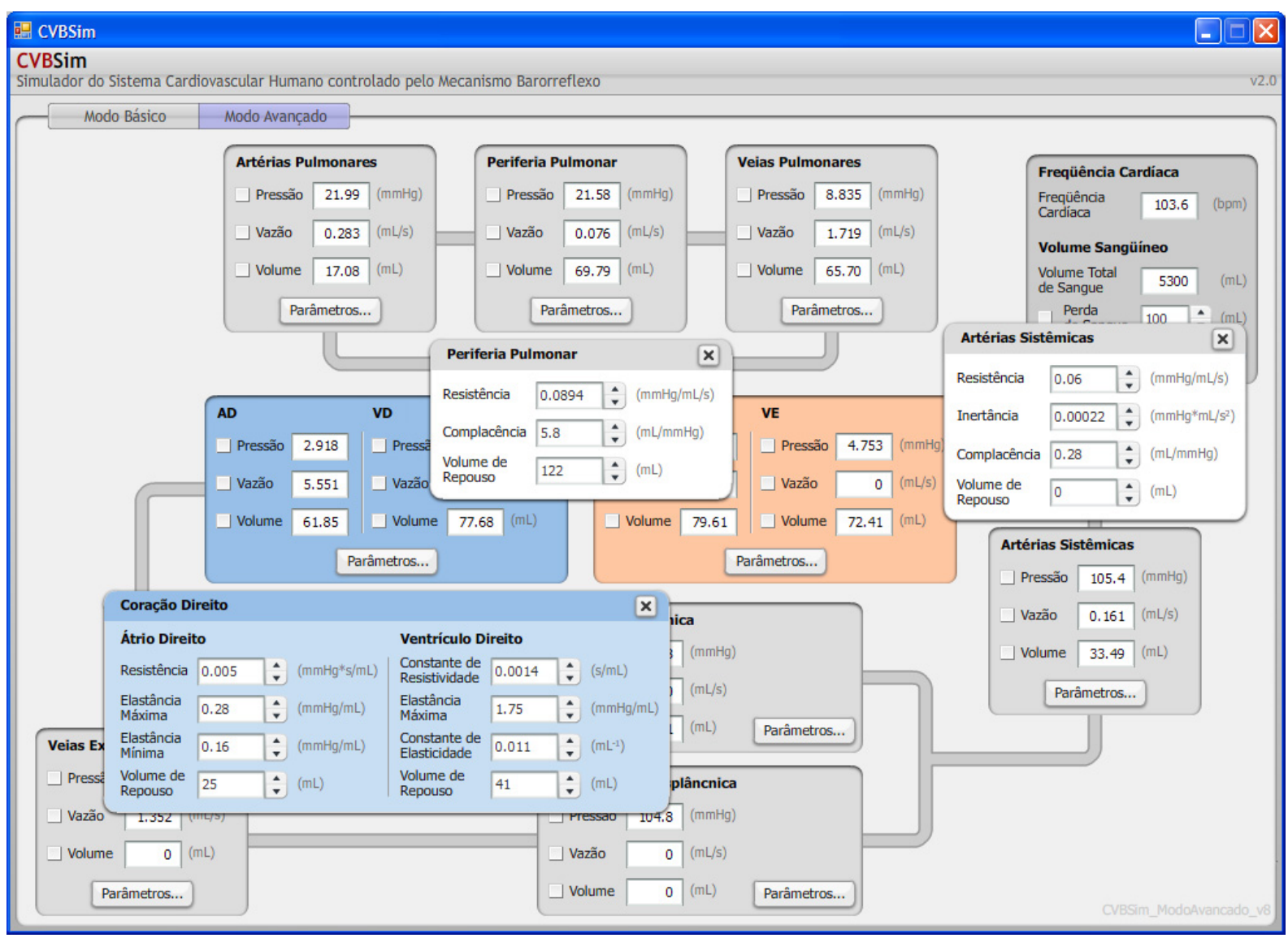

Figura 5-6 Exemplos de janelas de configuração de parâmetros, para artérias pulmonares (figura à esquerda) e para a periferia esplâncnica (figura à direita).

Uma janela de gráfico para o modo avançado é mostrada na Figura 5-7 abaixo, exemplificando a pressão nas artérias sistêmicas. O valor mostrado na caixa numérica à esquerda do eixo ordenado corresponde ao valor instantâneo do parâmetro plotado. Pode-se perceber na figura que foram utilizadas janelas do Windows com conteúdo desenhado através de código C\# para a plotagem dos gráficos. Isso foi feito para evitar a transmissão de dados entre as linguagens, potencializando o meIhor desempenho da linguagem C\# em relação ao Actionscript. 


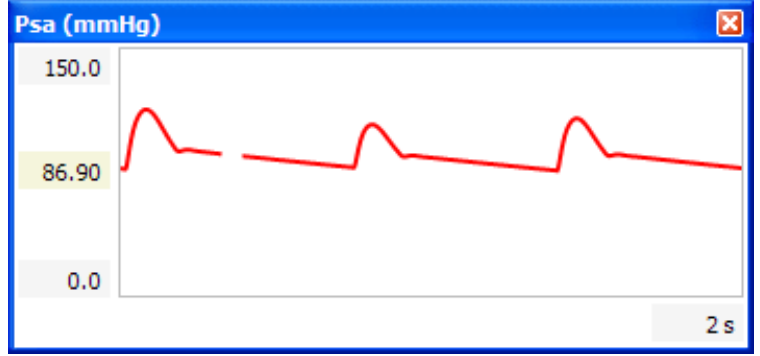

Figura 5-7 Janela de plotagem da curva de pressão ventricular esquerda. 


\section{Conclusões}

O objetivo original deste trabalho consistia no desenvolvimento de um simulador da fisiologia cardiovascular, que funcionaria com base em um modelo selecionado da literatura capaz de contabilizar todos os efeitos e parâmetros desejados e de representar de forma satisfatória o funcionamento do sistema. No entanto, como foi descrito ao longo do trabalho, o modelo inicialmente selecionado não atendia a todas as características especificadas, conforme indicaram as simulações numéricas realizadas. Percebendo que estas limitações relacionavam-se com a falta de atividade contrátil dos átrios, decidiu-se pela inclusão de um segundo objetivo neste trabalho, partindo-se para a modelagem da atividade atrial. É importante ressaltar que esta tarefa de modelagem acabou assumindo uma relevância equivalente à do simulador, não apenas para a finalização do trabalho, mas também como contribuição científica. Recorrendo novamente à literatura, foi selecionado como referência para o novo modelo um trabalho que já incluía os átrios ativos, mas que não fora anteriormente utilizado por não descrever os mecanismos de controle por barorreflexo, considerados necessários para a aplicação desejada.

De fato, a observação dos resultados obtidos permite que se conclua que as limitações encontradas no modelo inicial deviam-se à ausência de contratilidade nos átrios. A inclusão da atividade contrátil proporcionou significativa melhoria nos resultados, aproximando o modelo da teoria e de dados empíricos disponíveis. Ainda assim, algumas ressalvas precisam ser feitas, principalmente com relação às pressões atriais e às pressões venosas. Considerando como referência os dados extraídos da literatura mostrados no Capítulo 4 (Resultados das Simulações Numéricas), os valores de pressão obtidos nos átrios atingiram apenas cerca de $30 \%$ dos valores esperados, enquanto os resultados de pressão venosa central não chegaram aos $20 \%$. No entanto, a maior parte dos valores de referência apresenta variações, devido aos métodos de medição ou mesmo à fisiologia do próprio sistema, o que de certa forma compromete as comparações. Ainda assim, pode-se considerar que a origem das diferenças entre os resultados e a literatura está relacionada com os parâmetros utilizados. Por terem sido diretamente extraídos de trabalhos de terceiros, acredita-se que uma sintonia mais aprofundada possa 
sanar estes problemas, até porque as formas dos traçados resultantes estão bastante semelhantes aos teóricos. Além disso, na inclusão da contratilidade dos átrios, foram consideradas resistências constantes, e não foi considerada a atuação do sistema de controle por barorreflexo sobre os parâmetros atriais, como fora feito para os ventrículos. Estas decisões foram tomadas para simplificar o ajuste de parâmetros, por reduzir a quantidade de variáveis no sistema, mas sua alteração pode auxiliar na solução dos problemas mencionados. De qualquer forma, como já foi mencionado, pode-se considerar que o presente trabalho apresenta uma significativa contribuição na modelagem do sistema cardiovascular humano.

O modelo do mecanismo reflexo baroceptor foi utilizado conforme extraído da literatura, sem alterações de equações ou de parâmetros. Por este motivo, não foi executada uma verificação qualitativa muito rigorosa, pois o modelo original já mostrava resultados satisfatórios para a aplicação desejada (URSINO, 1998).

O desenvolvimento do simulador do sistema cardiovascular também enfrentou algumas adversidades, originadas da complexidade do próprio sistema simulado. Em comparação com o modelo utilizado anteriormente no simulador do sistema respiratório com ventilação forçada (LONARDONI; IWATA, 2002), o número de compartimentos cresceu de dois para 12, além das equações do sistema de controle por barorreflexo. Se antes já fora experimentada alguma lentidão com a plataforma selecionada - o Macromedia Flash - constatou-se que seu uso se torna inviável para um sistema de tamanha complexidade. Esta limitação motivou a busca por novas linguagens, isoladas ou combinadas entre si, no sentido de utilizar uma linguagem de maior desempenho (mais baixo nível) para os cálculos e outra de mais alto nível para a interface gráfica. Por fim, chegou-se a duas soluções com viabilidade técnica para uso no simulador - código em linguagem C++ com interface em Qt (biblioteca gráfica multiplataforma) e código em C\# com interface em Macromedia Flash. Devido às vantagens proporcionadas pelo Flash em comparação ao Qt para o desenvolvimento de elementos gráficos, a última solução (C\# com Macromedia Flash) prevaleceu sobre as demais, sendo a selecionada para a aplicação.

Devido à versatilidade destas combinações, o simulador resultante é capaz de receber a inclusão de uma enorme quantidade de eventuais recursos e melhorias, bem como ser adaptado para 
simulações diversas, em sistemas de complexidade relativamente elevada. A limitação fica por conta do algoritmo de integração utilizado, trapezoidal com intervalo de tempo fixo. O uso de um integrador mais eficiente poderia reduzir a carga computacional e aumentar o desempenho do simulador.

\subsection{Sugestões para trabalhos futuros}

O modelo do sistema cardiovascular apresentado neste trabalho permite evoluções de diversas formas, tanto no sentido do aumento no número de compartimentos quanto da inclusão de efeitos adicionais e sistemas de controle não considerados. No primeiro caso, é possível citar a individualização dos órgãos periféricos (para avaliação de efeitos mais localizados); a inclusão de rins, estômago e outros órgãos capazes de alterar a quantidade de sangue em circulação; a inclusão de circulações especiais, como a coronária e a cerebral. Para auxiliar pesquisas de dispositivos de auxílio à circulação sangüínea, pode-se mencionar, por exemplo, a verificação da influência da adição de uma bomba entre o ventrículo esquerdo e a aorta, para avaliação das alterações de vazão e pressão, ou ainda a inclusão de shunts entre os ventrículos. No caso do sistema de controle, existem ainda diversos mecanismos de regulação da pressão, seja em curto, médio ou longo prazo. Em um nível mais distante, pode-se até mesmo considerar a adição de trocas gasosas e de consumo de nutrientes. Além disso, antes mesmo da inclusão de novos efeitos, pode-se considerar a sintonia dos parâmetros do modelo e a inclusão de resistência variável e atuação neural nos átrios.

O simulador CVBSim também oferece diversas possibilidades de evolução, considerando-se como mais relevante para o uso didático, o desenvolvimento de tutoriais interativos que reajam às intervenções do operador. Pode-se ainda citar o desenvolvimento de conjuntos de pré-ajustes para patologias e casos clínicos, para eventual uso em salas de aula. Além disso, qualquer acréscimo ao modelo pode ser refletido no simulador, de modo a aumentar sua capacidade de representação da realidade. Com relação ao desempenho, a mencionada troca do integrador poderia proporcionar maior robustez ao aplicativo, possibilitando a inclusão de recursos mais pesados (como som e vídeo) sem comprometimento da carga computacional ou do desempenho das funções prioritárias. 


\section{Referências Bibliográficas}

ABRAHAMSON, S.; DENSON, J. S.; WOLF, R. M. Effectiveness of a simulator in training anesthesiology residents. Journal of Medical Education, v. 44, p. 515-519, 1969. Reimpresso por Quality and Safety in Health Care, v. 13; p. 395-399, 2004.

ALJURI, N.; COHEN, R. J. Theoretical considerations in the dynamic closed-loop baroreflex and autoregulatory control of total peripheral resistance. American Journal of Physiology: Heart and Circulatory Physiology, v. 287, p. H2252-H2273, 2003.

ANTONI, H. Functional properties of the heart. In: GREGER, R.; WINDHORST, U. (Eds.). Comprehensive human physiology: From cellular mechanics to integration. Vol. 2. Berlin: SpringerVerlag, 1996a, p. 1801-1823.

. The cardiac function cycle. In: GREGER, R.; WINDHORST, U. (Eds.). Comprehensive human physiology: From cellular mechanics to integration. Vol. 2. Berlin: Springer-Verlag, 1996b, p. $1793-1800$.

BERGER, S. A.; GOLDSMITH, W.; LEWIS, E. R. (Eds.). Introduction to Bioengineering. New York: Oxford University Press, 1996, 526 p.

BEYEA, S. C.; KOBOKOVICH, L. J. Human patient simulation: A teaching strategy. AORN Journal, v. 80, p. 738-742, 2004.

BRAUNWALD, E. Tratado de medicina cardiovascular: Volume 1. Quarta edição. São Paulo: Roca, 1996. 949 p.

BULLOCK, J.; BOYLE III, J.; WANG, M. B. Physiology. Terceira edição. Media: Williams \& Wilkins, 1995. (The National Medical Series for Independent Study).

de CAÑETE, J. F.; de VEGA, D. G.; DAVID, M.; GARCÍA-CEREZO, A. Cardiovascular control using artificial neuronal structures: Modeling the baroreceptor regulation mechanism. Neurocomputing, $v$. 43, p. 37-50, 2002.

CAVALCANTI, S.; CAVANI, S.; CIANDRINI, A.; AVANZOLINI, G. Mathematical modeling of arterial pressure response to hemodialysis-induced hypovolemia. Computers in Biology and Medicine, 2005. No prelo.

CAVAZZA, M.; SIMO, A. A Virtual Patient Based on Qualitative Simulation. In: INTERNATIONAL CONFERENCE ON INTELLIGENT USER INTERFACES, 8., 2003, Miami. Proceedings of the 8th international conference on Intelligent user interfaces. New York: ACM Press, 2003. p. 1925.

CRAIEM, D.; ARMENTANO, R. The new apparent compliance concept as a simple lumped model. Cardiovascular Engineering: An International Journal, v. 3, p. 81-83, 2003.

DEFONTAINE, A.; HERNÁNDEZ, A.; CARRAULT, G. Multi-formalism modeling and simulation: application to cardiac modeling. Acta Biotheoretica, Holanda, v. 52, p. 273-290, 2004.

FINK, M.; BATZEL, J. J.; KAPPEL, F. An optimal control approach to modeling the cardiovascularrespiratory system: An application to orthostatic stress. Cardiovascular Engineering: An International Journal, v. 4, p. 27-38, 2004.

FORMAGGIA, L.; NOBILE, F.; QUARTERONI, A.; VENEZIANI, A. Multiscale modeling of the circulatory system: a preliminary analysis. Computing and Visualization in Science, v. 2, p. 75-83, 1999. 
FOX, S. I. Human Physiology. Nona edição. New York: McGraw-Hill Higher Education, 2006.

FUNG, Y. C. Biomechanics: Circulation. Segunda edição. New York: Springer-Verlag, 1997.

GABA, D. M. The future vision of simulation in health care. Quality and Safety in Health Care, v. 13 (Suppl. 1), p. i2-i10, 2004.

GALLAGHER, A. G.;RITTER, E. M.; CHAMPION, H.; HIGGINS, G.; FRIED, M. P.; MOSES, G.; SMITH, C. D.; SATAVA, R. M. Virtual reality simulation for the operating room: Proficiency-based training as a paradigm shift in surgical skills training. Annals of Surgery, v. 241, p. 364-372, 2005.

GERSTING, J. M.; ROTHE, C. F. Cardiovascular interactions tutorial: Architecture and design. Journal of Medical Systems, v. 26, p. 29-38, 2002.

GOODWIN, J. A.; VAN MEURS, W. L.; SÁ COUTO, C. S.; BENEKEN, J. E. W.; GRAVES, S. A. A model for educational simulation of infant cardiovascular physiology. Anesth Analg, v. 99, p. 16551664, 2004.

GREENE, A. S.; SHOUKAS, A. A. Changes in canine cardiac function and venous return curves by the carotid baroreflex. American Journal of Physiology: Heart and Circulatory Physiology, v. 251, p. H288-H296, 1986.

GUARINI, M.; URZÚA, J.; CIPRIANO, A. Estimation of cardiac function from computer analysis of the arterial pressure waveform. IEEE Transactions on Biomedical Engineering, v. 45, 14201428, 1998.

GUYTON, A. C.; HALL, J. E. Tratado de fisiologia médica. Nona edição. Rio de Janeiro: Guanabara Koogan, 1997.

HAWLEY, R. R.; DODGE, H. T.; GRAHAM, T. P. Left atrial volume and its changes in heart disease. Circulation, v. 34, p. 989-996, 1966.

HELDT, T.; CHANG, J. L.; CHEN, J. J. S.; VERGHESE, G. C.; MARK, R. G. Cycle-averaged dynamics of a periodically driven, closed-loop circulation model. Control Engineering Practice, v. 13, p. 1163-1171, 2005.

HUGHSON, R. L.; O'LEARY, D. D.; SHOEMAKER, J. K.; LIN, D. C.; TOPOR, Z. L.; EDWARDS, M. R.; TULPPO, M. P. Searching for the vascular component of the arterial baroreflex. . Cardiovascular Engineering: An International Journal, v. 4, p. 155-162, 2004.

KAYE, J. M.; PRIMIANO, F. P.; METAXAS, D. N. A three-dimensional virtual environment for modeling mechanical cardiopulmonary interactions. Medical Image Analysis, Oxford, v. 2, p. 169-195, 1998.

KARAKAWA, M.; IGARASHI, K. A mathematical approach to cardiovascular disease: Mechanics of blood circulation. Primeira edição. Japan: Kokuseido Publishing, 1998. 152 p.

KAREMAKER, J. M. Neurophysiology of the baroreceptor reflex. In: KITNEY, R. I.; ROMPELMAN, O. (Eds.). The beat-by-beat investigation of cardiovascular function: Measurement, analysis and applications. New York: Oxford University Press, 1987. Cap. 2, p. 27-49.

KATONA, P. G.; POITRAS, J. W.; BARNETT, G. O.; TERRY, B. S. Cardiac vagal efferent activity and heart period in the carotid sinus reflex. American Journal of Physiology, v. 218, p. 10301037, 1970.

KELMAN, G. R. Applied cardiovascular physiology. Segunda edição. London: Butterworths, 1997.

LAGANÀ, K.; BALOSSINO, R.; MIGLIAVACCA, F.; PENNATI, G.; BOVE, E. L.; DE LEVAL, M. R.; DUBINI G. Multiscale modeling of the cardiovascular system: Applications to the study of pulmonary and coronary perfusions in the univentricular circulation. Journal of Biomechanics, v. 38, p. 11291141, 2005. 
LANFRANCHI, P. A.; SOMERS, V. K. Arterial baroreflex function and cardiovascular variability: Interactions and implications. American Journal of Physiology: Regulatory, Integrative and Comparative Physiology, v. 283, p. R815-R826, 2002.

LANGLEY, L. L. Physiology of Man. $4^{\mathrm{a}}$ ed. New York: Van Nostrand Reinhold Company, 1971.

LI, X.; BAI, J.; CUI, S.; WANG, S. Simulation study of the cardiovascular functional status in hypertensive situation. Computers in Biology and Medicine, v. 32, p. 345-362, 2002.

LIPOWSKY, H. H. Shear stress in the circulation. In: BEVAN, J. A.; KALEY, G.; RUBANYI, G. M. (Eds.). Flow dependent regulation of cardiovascular function. New York: Oxford University Press, 1995 (Clinical Physiology Series - American Physiological Society).

LIU, H. K.; GUILD, S. J.; RINGWOOD, J. V.; BARRETT, C. J.; LEONARD, B. L.; NGUANG, S. K.; NAVAKATIKYAN, M. A.; MALPAS, S. C. Dynamic baroreflex control of blood pressure: Influence of the heart vs. peripheral resistance. American Journal of Physiology: Regulatory, Integrative and Comparative Physiology, v. 283, p. R533-R542, 2002.

LONARDONI, J. A. C.; IWATA, L. P. Desenvolvimento de um modelo multicompartimental do sistema respiratório humano. 2002. 162 f. Trabalho de conclusão de curso - Departamento de Engenharia Mecânica, Escola Politécnica da Universidade de São Paulo, São Paulo, 2002.

MAGOSSO, E.; BIAVATI, V.; URSINO, M. Role of the baroreflex in cardiovascular instability: A modeling study. Cardiovascular Engineering, v. 1, p. 101-115, 2001.

MAGOSSO, E.; URSINO, M. A mathematical model of $\mathrm{CO}_{2}$ effect on cardiovascular regulation. American Journal of Physiology: Heart and Circulatory Physiology, v. 281, p. H2036-H2052, 2001.

MANIAR, H. S.; PRASAD, S. M.; GAYNOR, S. L.; CHU, C. M.; STEENDIJK, P.; MOON, M. R. American Journal of Physiology: Heart and Circulatory Physiology, v. 284, p. 350-357, 2003.

MATSUDA, Y.; TOMA, Y.; OGAWA, H.; MATSUZAKI, M.; KATAYAMA, K.; FUJII, T.; YOSHINO, F.; MORITANI, K.; KUMADA, T.; KUSUKAWA, R. Importance of left atrial function in patients with myocardial infarction. Circulation, v. 67; p. 566-571, 1983.

van MEURS, W. L.; GOOD, M. L.; LAMPOTANG, S. Functional anatomy of full-scale patient simulators. Journal of Clinical Monitoring, v. 13, p. 317-324, 1997.

van MEURS, W. L.; SÁ COUTO, P. M.; SÁ COUTO, C. D.; BERNARDES, J. F.; AYRES-DE-CAMPOS, D. Development of foetal and neonatal simulators at the University of Porto. Medical Education, $\mathrm{v}$. 37 (Suppl. 1), p. 29-33, 2003.

MICHAEL, J. In pursuit of meaningful learning. Advances in Physiology Education, v. 25, p.145$158,2001$.

MICHAEL, L. The design of fluid transport systems: A comparative perspective. In: BEVAN, J. A.; KALEY, G.; RUBANYI, G. M. (Eds.). Flow dependent regulation of cardiovascular function. New York: Oxford University Press, 1995 (Clinical Physiology Series - American Physiological Society).

MILLER, M. J.; McKAY, R. G.; FERGUSON, J. J.; SAHAGIAN, P.; NAKAO, S.; COME, P. C.; GROSSMAN, W. Right atrial pressure-volume relationships in tricuspid regurgitation. Circulation, v. 73, p. 799-808, 1986.

MUKKAMALA, R.; COHEN, R. J. A forward model-based validation of cardiovascular system identification. American Journal of Physiology: Heart and Circulatory Physiology, v. 281, p. H2714-H2730, 2001.

MUKKAMALA, R.; TOSKA, K.; COHEN, R. J. Noninvasive identification of the total peripheral resistance baroreflex. American Journal of Physiology: Heart and Circulatory Physiology, v. 284, p. H947-H959, 2003. 
NAKAO, M.; OYAMA, H.; KOMORI, M.; MATSUDA, T.; SAKAGUCHI, G.; KOMEDA, M.; TAKAHASHI, T. Haptic reproduction and interactive visualization of a beating heart for cardiovascular surgery simulation. International Journal of Medical Informatics, v. 68, p. 155-163, 2002.

OLUFSEN, M. S.; NADIM, A. On deriving lumped models for blood flow and pressure in the systemic arteries. Mathematical Biosciences and Engineering, v. 1, p. 61-80, 2004.

OOMEN, B.; KARAMANOGLU, M.; KOVÁCS, S. J. Modeling time varying elastance: The meaning of "load-independence". Cardiovascular Engineering: An International Journal, v. 3, p. 123130, 2003.

OTTESEN, J. T. Modeling of the baroreflex-feedback mechanism with time-delay. Journal of Mathematical Biology, v. 36, p. 41-63, 1997.

OTTESEN, J. T. Modeling the dynamical baroreflex-feedback control. Mathematical and Computer Modeling, v. 31, p. 167-173, 2000.

PAGEL, P. S.; KEHL, F.; GARE, M.; HETTRICK, D. A.; KERSTEN, J. R.; WARLTIER, D. C. Mechanical function of the left atrium: New insights based on analysis of pressure-volume relations and doppler echocardiography. Anesthesiology, v. 98, p. 975-994, 2003.

PENNATI, G.; BELLOTTI, M. FUMERO, R. Mathematical modeling of the human foetal cardiovascular system based on Doppler ultrasound data. Medical Engineering \& Physics, v. 19, p. 327-335, 1997.

PODNAR, T.; RUNOVC, F.; KORDAŠ, M. Simulation of cardiovascular physiology: The diastolic function(s) of the heart. Computers in Biology and Medicine, v. 32, p. 363-377, 2002.

POTTS, J. T.; HATANAKA, T.; SHOUKAS, A. A. Effect of arterial compliance on carotid sinus baroreceptor reflex control of the circulation. American Journal of Physiology: Heart and Circulatory Physiology, v. 270, p. H988-H1000, 1996.

QUICK guide to cardiopulmonary care. Irvine: Edwards Lifesciences LLC, 2002. 115p.

RAY, C. D. Medical engineering. Chicago: Year BookMedical Publishers, 1974.

RHOADES, R. A.; TANNER, G. A. Medical Physiology. Segunda edição. Lippincott Williams \& Wilkins, 2003.

di RIENZO, M.; PARATI, G.; MANCIA, G.; PEDOTTI, A.; CASTIGLIONI, P. Investigating baroreflex control of circulation using signal processing techniques. IEEE Engineering in Medicine and Biology, v. 16, p. 86-95, 1997.

ROTHE, C. F. Reflex control of veins and vascular capacitance. Physiological Reviews, Estados Unidos, v. 63, p. 1281-1342, 1983.

ROTHE, C. F.; GERSTING, J. M. Cardiovascular interactions: An interactive tutorial and mathematical model. Advances in Physiology Education, v. 26, p. 98-109, 2002.

ROWELL, L. B.; DETRY, JM. R.; BLACKMON, J. R.; WYSS, C. Importance of the splanchnic vascular bed in human blood pressure regulation. Journal of Applied Physiology, v. 32 (2), p. 213220, 1972.

RUPNIK, M.; RUNOVC, F.; SKET, D.; KORDAŠ, M. Cardiovascular physiology: Simulation of steady state and transient phenomena by using the equivalent electronic circuit. Computer Methods and Programs in Biomedicine, v. 67, p. 1-12, 2002.

SÁ COUTO, D.; van MEURS, W. L.; GOODWIN, J. A. Graphical and mathematical representation of congenital heart disease. European Journal of Anaesthesiology, v. 20, p. 841-842, 2003.

SCHMIDT, R. M.; KUMADA, M.; SAGAWA, K. Cardiovascular responses to various pulsatile pressures in the carotid sinus. American Journal of Physiology, v. 223, p. 1-7, 1972. 
STERGIOPULOS, N.; WESTERHOF, B. E.; WESTERHOF, N. Total arterial inertance as the fourth element of the windkessel model. American Journal of Physiology: Heart and Circulatory Physiology, v. 276, p. H81-H88, 1999.

STERN, R. H,; RASMUSSEN, H. Left ventricular ejection: Model solution by collocation, an aproximate analytical method. Computers in Biology and Medicine, v. 26, p. 255-261, 1996.

URBASZEK, A.; SCHALDACH, M. Hemodynamic aspects of rate-responsive pacing: A simulation study. Advances in Engineering Software, v. 28, p. 385-393, 1997.

URSINO, M. Interaction between carotid baroregulation and the pulsating heart: A mathematical model. American Journal of Physiology: Heart and Circulatory Physiology, v. 275, p. H1733-H1747, 1998.

URSINO, M.; ANTONUCCI, M.; BELARDINELLI, E. Role of active changes in venous capacity by the carotid baroreflex: Analysis with a mathematical model. American Journal of Physiology: Heart and Circulatory Physiology, v. 267, p. H2531-H2546, 1994.

URSINO, M.; FIORENZI, A.; BELARDINELLI, E. The role of pressure pulsatility in the carotid baroreflex control: A computer simulation study. Computers in Biology and Medicine, v. 26, p. 297-314, 1996.

URSINO, M.; MAGOSSO, E. Acute cardiovascular response to isocapnic hypoxia: I - A mathematical model. American Journal of Physiology: Heart and Circulatory Physiology, v. 279, p. H149-H165, 2000a.

URSINO, M.; MAGOSSO, E. Acute cardiovascular response to isocapnic hypoxia: II - Model validation. American Journal of Physiology: Heart and Circulatory Physiology, v. 279, p. H166H175, 2000b.

URSINO, M.; MAGOSSO, E. Role of short-term cardiovascular regulation in heart period variability: A modeling study. American Journal of Physiology: Heart and Circulatory Physiology, v. 284, p. H1479-H1493, 2003.

van de VOSSE, F. N. Mathematical modeling of the cardiovascular system. Journal of Engineering Mathematics, Netherlands, v. 47, p. 175-183, 2003.

WHITE, R. J.; FITZJERRELL, D. G.; CROSTON, R. C. Fundamentals of lumped compartmental modeling of the cardiovascular system. In: GHISTA, D. N.; van VOLLENHOVEN, E.; YANG, W. J.; REUL, H.; BLEIFELD, W. (Eds.). Cardiovascular Engineering: Part 1 - Modeling. Basel: Karger, 1983. p. 162-184 (Advances in Cardiovascular Physics, v. 5).

WHITE, R. J.; CROSTON, R. C.; FITZJERRELL, D. G. Cardiovascular modeling: Simulating the human response to exercise, lower body negative pressure, zero gravity and clinical conditions. In: GHISTA, D. N.; van VOLLENHOVEN, E.; YANG, W. J.; REUL, H.; BLEIFELD, W. (Eds.). Cardiovascular Engineering: Part 1 - Modeling. Basel: Karger, 1983. p. 195-229 (Advances in Cardiovascular Physics, v. 5).

WOODRUFF, E. A.; MARTIN, J. F.; OMENS, M. A model for the design and evaluation of algorithms for closed-loop cardiovascular therapy. IEEE Transactions on Biomedical Engineering, v. 44, p. 694-705, 1997.

ŽÁČEK, M.; KRAUSE, E. Numerical simulation of the blood flow in the human cardiovascular system. Journal of Biomechanics, v. 29, p. 13-20, 1996.

\section{Material Eletrônico}

CARDIOVASCULAR PHYSIOLOGY CONCEPTS. Sob responsabilidade de Richard E. Klaubunde. Disponível em: <http://www.cvphysiology.com/index.html>. Acesso em: Agosto de 2005. 
CIRCSIM-Tutor Project. Sob responsabilidade de: Department of Computer Science, Illinois Institute of Technology \& Department of Physiology, Rush College of Medicine. Disponível em: <http://www.cs.iit.edu/ circsim/>. Acesso em: Agosto de 2005.

PHYSIONET - The research resource for complex physiologic signals. Bethesda, USA: National Center for Research Resources. Disponível em: <http://www.physionet.org/>. Último acesso em: Abril de 2006.

RCVSIM - A Cardiovascular Simulator For Research. Publicado em: PhysioNet.org, sob responsabilidade de Harvard-MIT Division of Health Sciences and Technology, Massachussets Institute of Technology, Cambridge. Disponível em: <http://www.physionet.org/physiotools/rcvsim/>. Acesso em: Março de 2005. 


\section{Apêndice A. Classificações da Simulação}

\section{A.1 As 11 Dimensões da Simulação}

Gaba introduziu em 2004 uma classificação que enquadra dentro de 11 dimensões todas as aplicações da simulação, atuais ou futuras. Apenas algumas destas dimensões se aplicam ao presente trabalho, mas todas serão descritas, em caráter informativo.

1) A finalidade e os objetivos da atividade de simulação.

a) Educação. Ênfase nos conceitos fundamentais, no conhecimento e nas competências básicos; serve de introdução ao objeto real de trabalho.

b) Treinamento. Foca nas tarefas e no trabalho reais.

c) Avaliação de desempenho e competência de equipes e clínicos individuais.

d) Ensaios clínicos. Ênfase na prática de procedimentos complexos.

e) Pesquisa e investigação de fatores humanos e usabilidade.

2) A unidade de participação na simulação.

a) Individual.

b) Em grupos de indivíduos, sem delimitação específica de funções.

c) Em equipes, com delimitação específica de funções.

d) Em unidades de trabalho, compostas por equipes, como em uma UTI.

e) Organizacional. Inclui participantes de esferas não apenas clínicas, mas também organizacionais.

3) O nível de experiência dos participantes da simulação.
a) Escola. Primária ou secundária.
b) Universidade.
c) Educação profissional inicial.
d) Residência ou treinamento em serviço. 
e) Educação continuada e treinamento.

4) O escopo clínico em que a simulação é aplicada.
a) Imagem. Interpretação de diagnósticos.
b) Cuidados básicos, psiquiatria.
c) Hospitalar.
d) Procedural. Cirurgias.
e) Dinâmico, de alto risco. UTI, primeiros socorros.

5) A categoria profissional dos participantes.
a) Auxiliares.
b) Técnicos.
c) Enfermeiras.
d) Médicos.
e) Gerentes, executivos.
f) Governantes, legisladores.

6) O tipo de conhecimento, habilidade ou comportamento alvo da simulação.
a) Compreensão de conceitos.
b) Habilidades técnicas.
c) Tomada de decisão.
d) Atitudes e comportamentos. Para trabalhos em equipe.

7) A idade do paciente simulado.
a) Neonatos.
b) Pediátricos.
c) Adolescentes.
d) Adultos.
e) Idosos. 
8) As tecnologias aplicadas ou requeridas.
a) Verbal, representação de papéis.
b) Pacientes padronizados. Atores.
c) Realidade virtual.
d) Paciente computacional. Simulação baseada em telas.
e) Paciente eletrônico. Simulação em escala natural.

9) O local de participação.
a) Domicílio ou escritório. Simulação baseada em telas.
b) Escola ou biblioteca. Simulação baseada em telas.
c) Laboratório dedicado. Realidade virtual.
d) Réplica de ambiente clínico.
e) O próprio local de aplicação.

10) A extensão da participação direta na simulação.
a) Observação remota. Não interativa.
b) Observação remota com interação verbal.
c) Observação remota com interação simulada.
d) Interação direta.
e) Participação imersiva.

11) O método de acompanhamento da simulação.
a) Nenhum.
b) Crítica automática pelo simulador.
c) Crítica pré-armazenada.
d) Crítica em tempo de simulação.
e) Avaliação posterior por vídeo. 
Apêndice B. Diagramas de Blocos do Modelo com Átrios Ativos

Sistema Cardiovascular

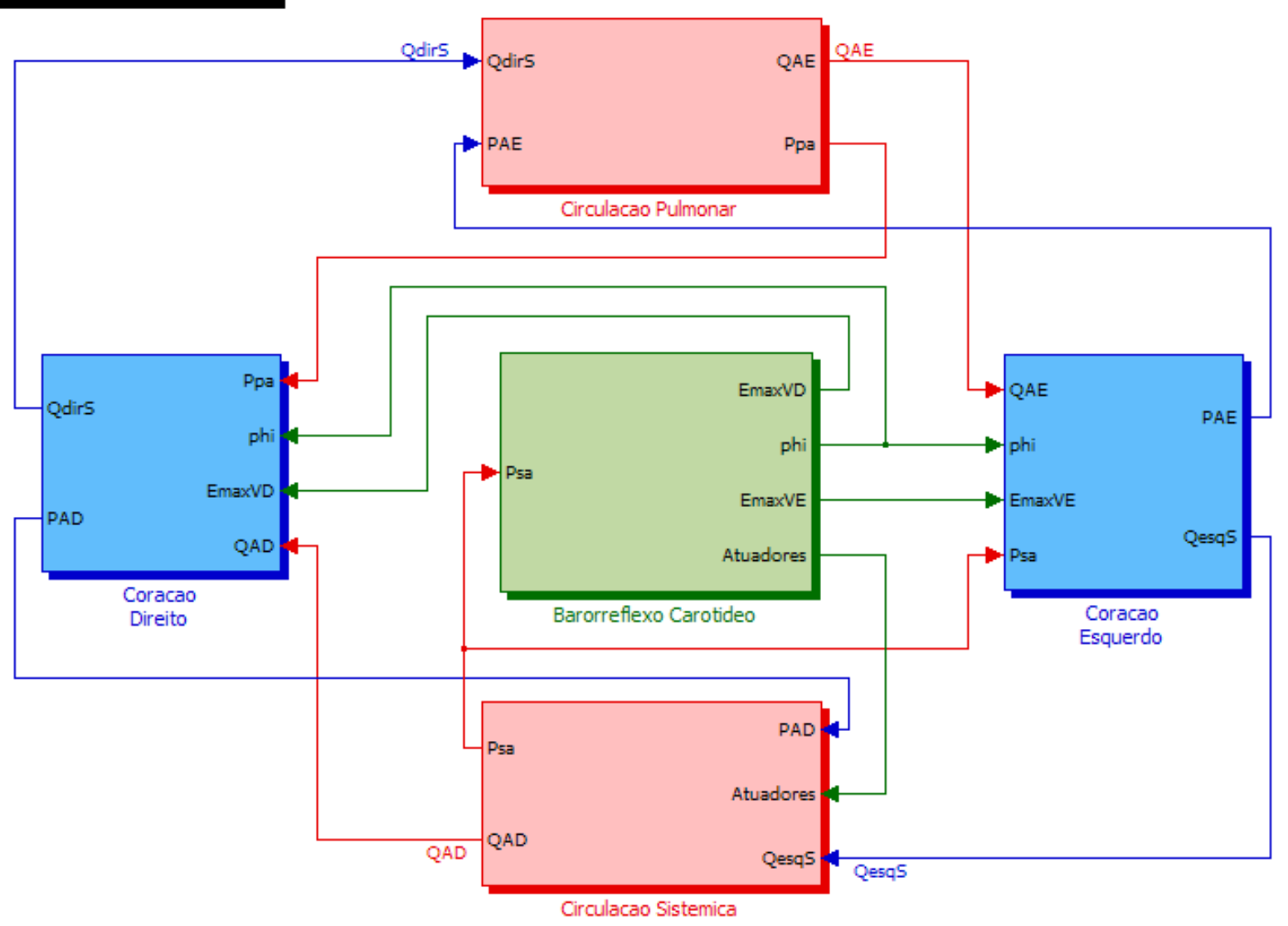

Apêndice B 1 Diagrama de blocos principal. 
Coração Esquerdo

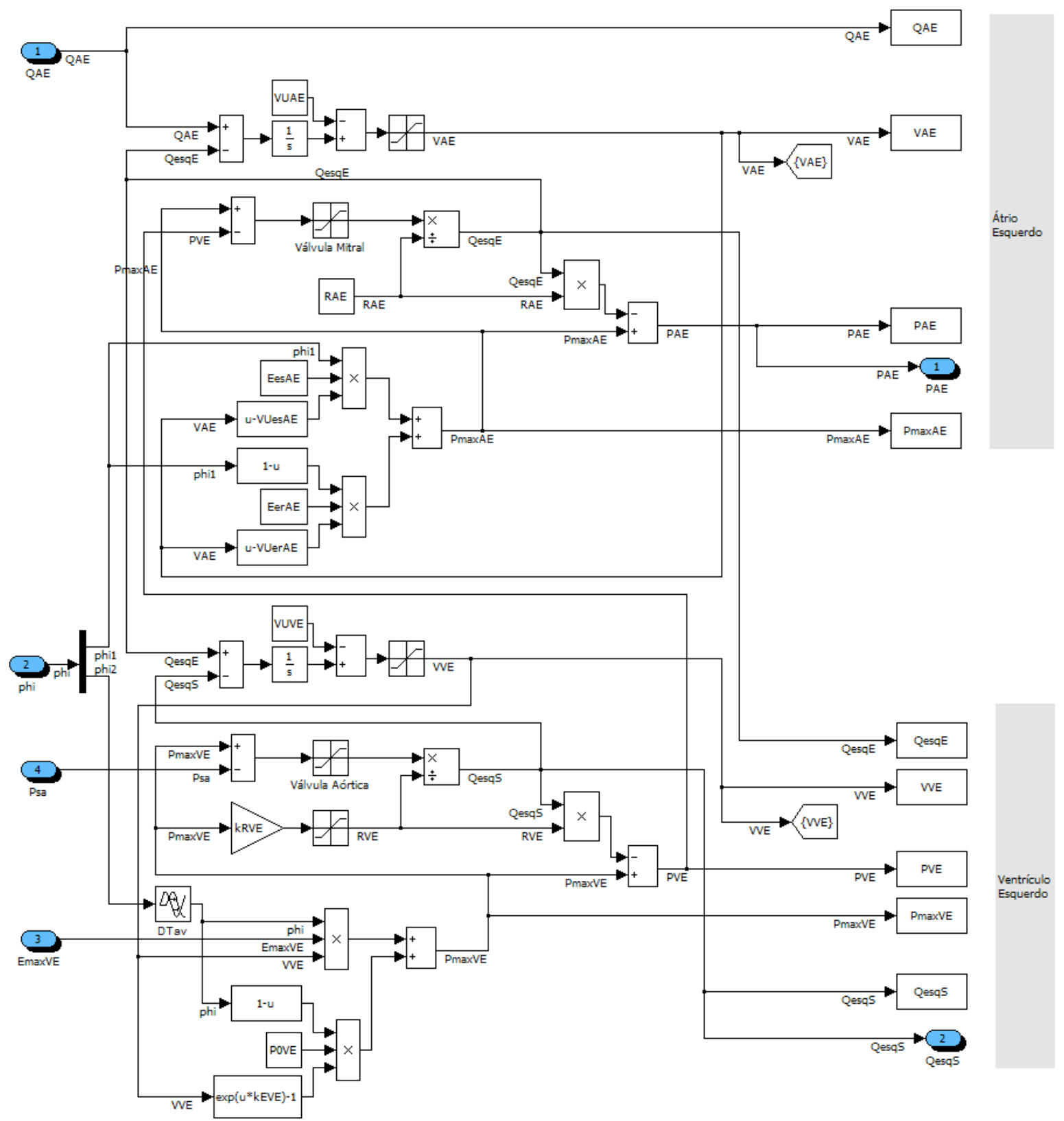

Apêndice B 2 Subconjunto do Coração Esquerdo. 


\section{Coração Direito}

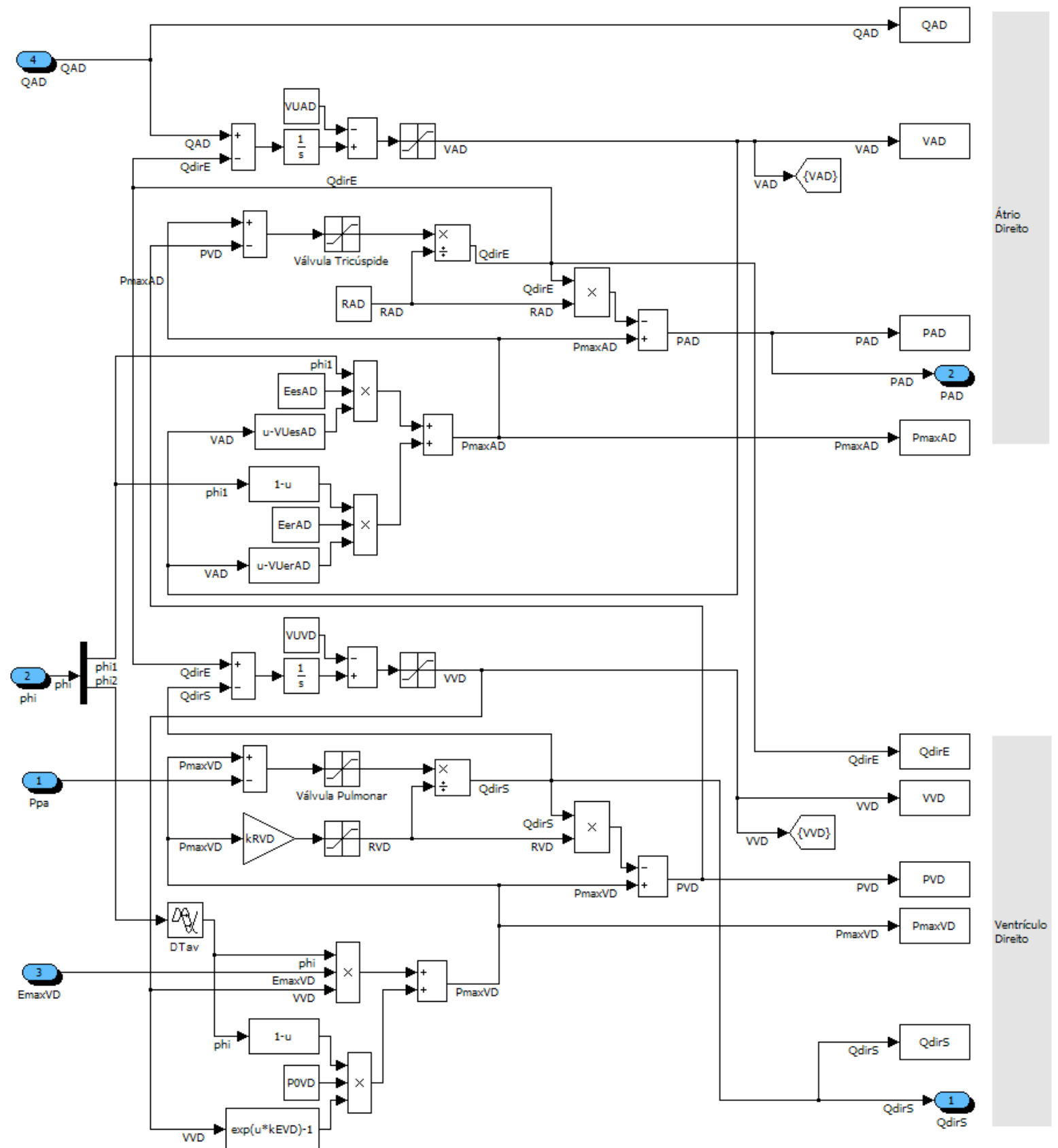

Apêndice B 3 Subconjunto do Coração Direito 


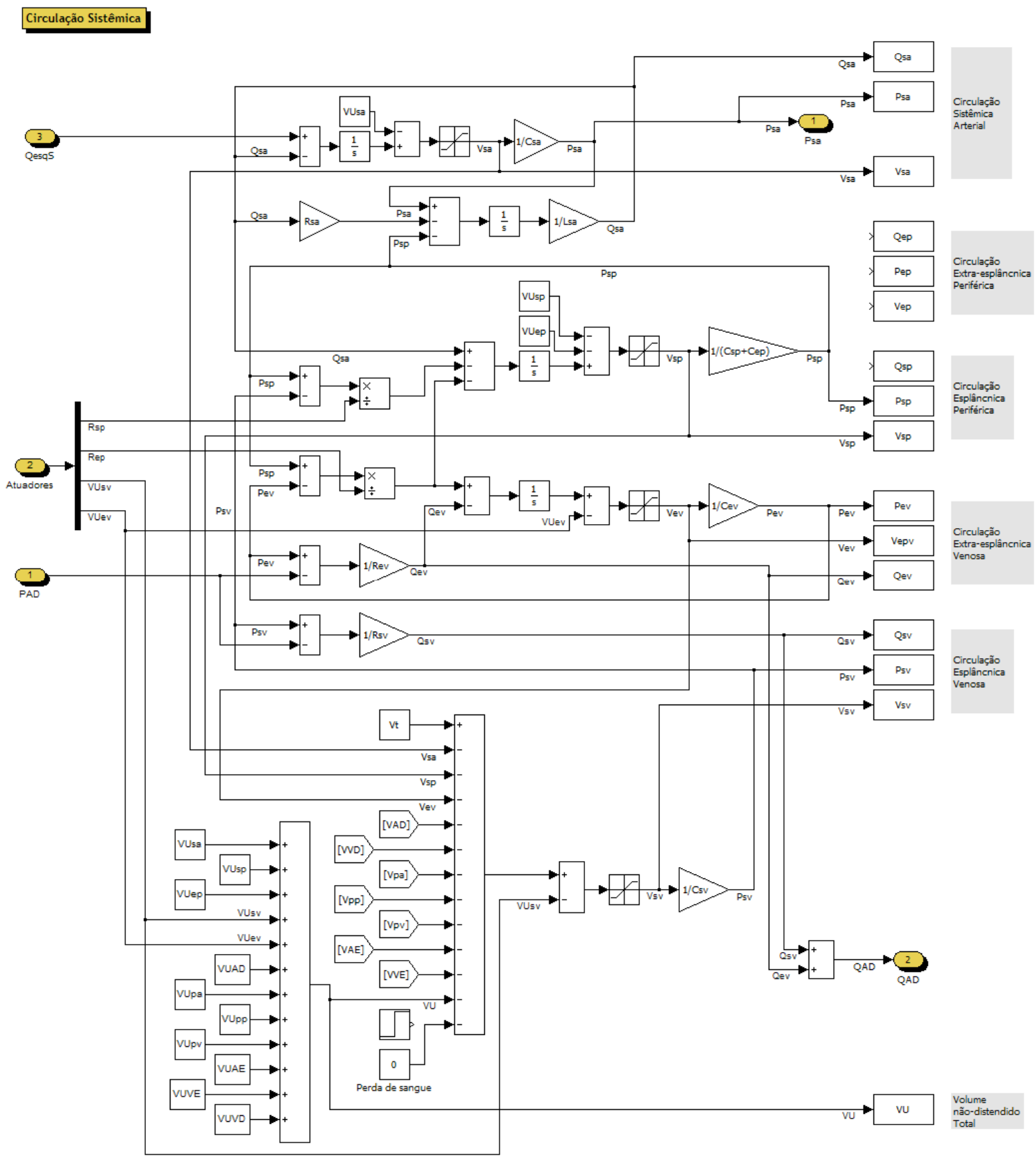

Apêndice B 4 Subconjunto da Circulação Sistêmica 
Circulação Pulmonar

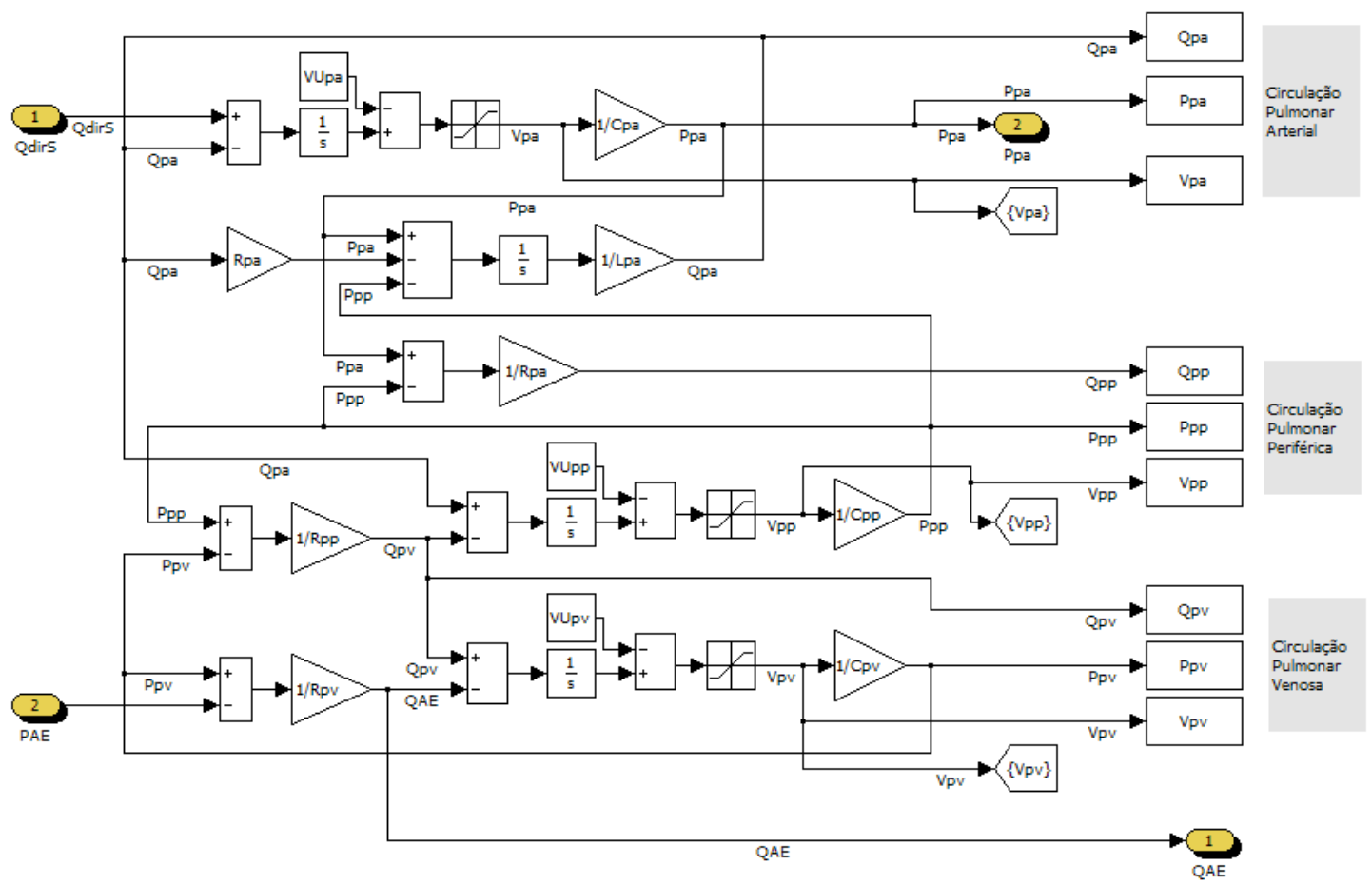

Apêndice B 5 Subconjunto da Circulação Pulmonar.

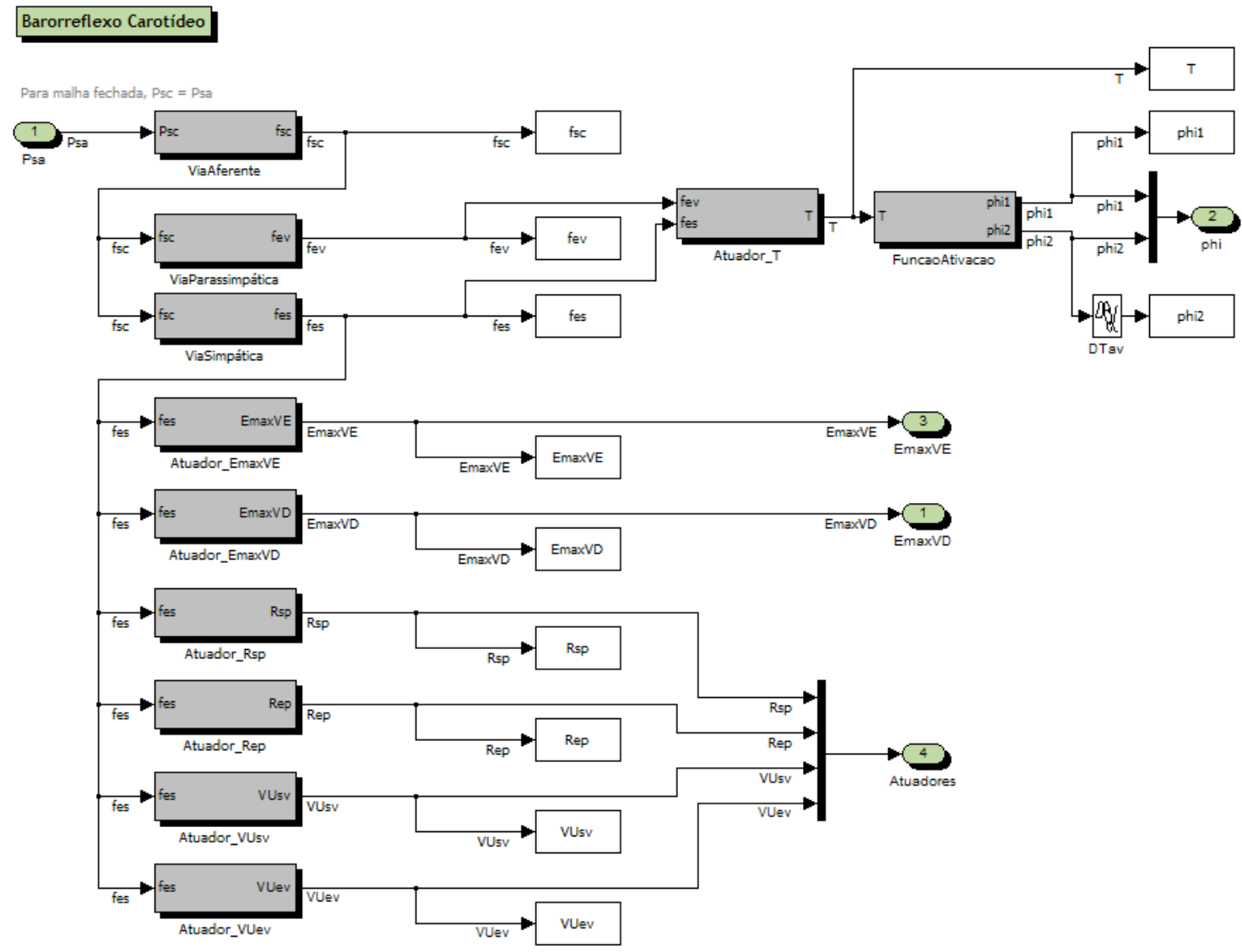

Apêndice B 6 Subconjunto do Barorreflexo Carotídeo. 


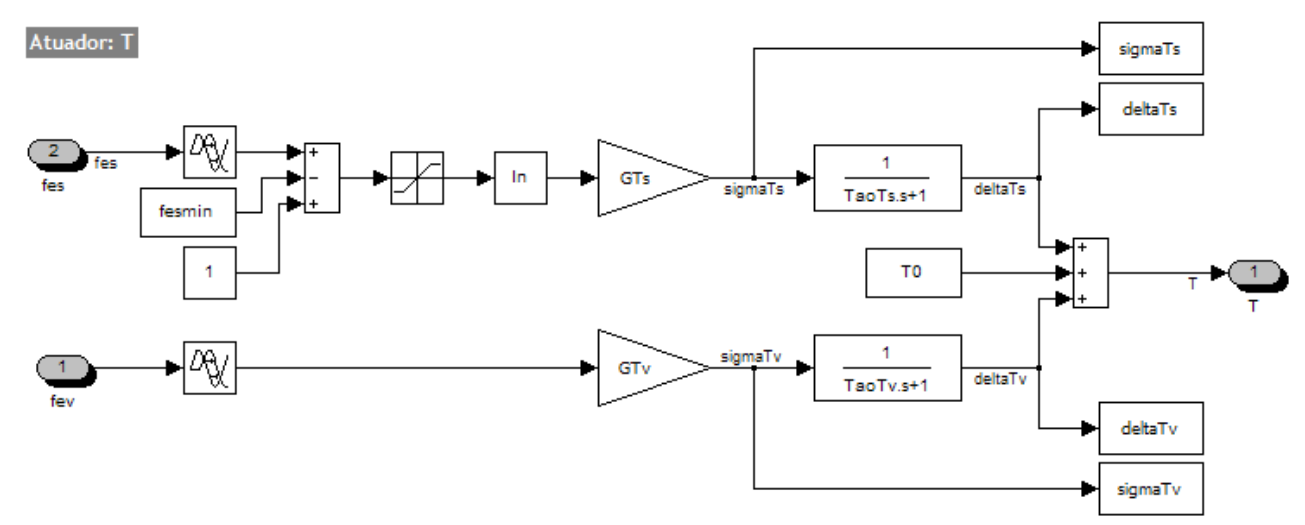

Apêndice B 7 Subconjunto para determinação do Período Cardíaco.

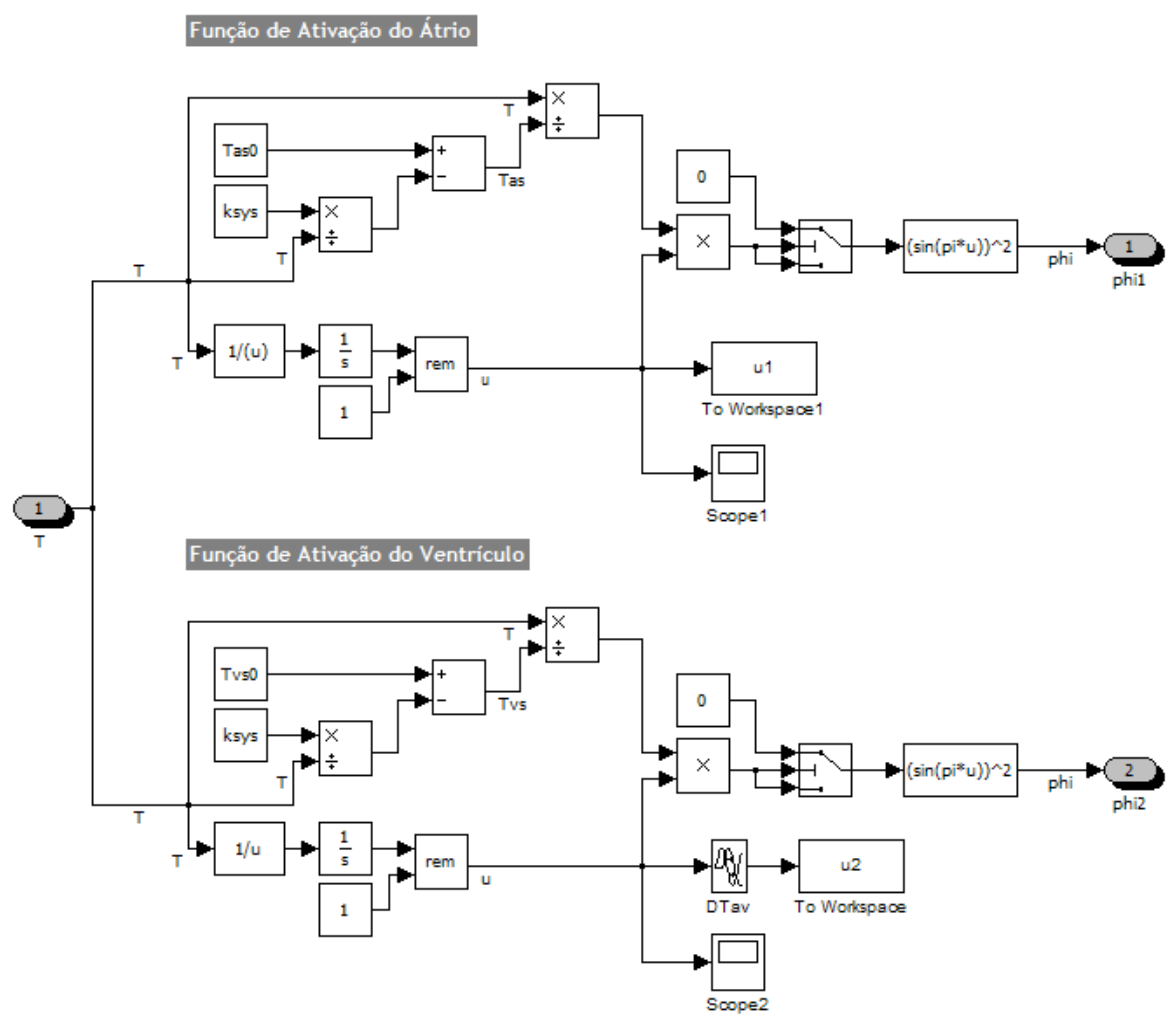

Apêndice B 8 Subconjunto da função de ativação. 